\title{
Aeroservoelasticity of articulated rotor hubs
}

by

\section{Derek Gransden}

\author{
A Dissertation submitted to \\ the Faculty of Graduate Studies and Research \\ in partial fulfilment of \\ the requirements for the degree of \\ Doctor of Philosophy \\ Ottawa-Carleton Institute for \\ Mechanical and Aerospace Engineering
}

Department of Mechanical and Aerospace Engineering

Carleton University

Ottawa, Ontario, Canada

September 2011

Copyright (C)

2011 - Derek Gransden 


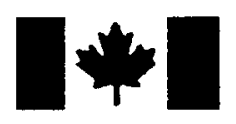

\author{
Library and Archives \\ Canada \\ Published Heritage \\ Branch \\ 395 Wellington Street \\ Ottawa ON K1A 0N4 \\ Canada
}

Bibliothèque et

Archives Canada

Direction du

Patrimoine de l'édition

395, rue Wellington

Ottawa ON K1A ON4

Canada
Your file Votre référence
ISBN: $978-0-494-83212-7$
Our file Notre référence
ISBN: $978-0-494-83212-7$
NOTICE:

The author has granted a nonexclusive license allowing Library and Archives Canada to reproduce, publish, archive, preserve, conserve, communicate to the public by telecommunication or on the Internet, loan, distribute and sell theses worldwide, for commercial or noncommercial purposes, in microform, paper, electronic and/or any other formats.

The author retains copyright ownership and moral rights in this thesis. Neither the thesis nor substantial extracts from it may be printed or otherwise reproduced without the author's permission.
AVIS:

L'auteur a accordé une licence non exclusive permettant à la Bibliothèque et Archives Canada de reproduire, publier, archiver, sauvegarder, conserver, transmettre au public par télécommunication ou par l'Internet, prêter, distribuer et vendre des thèses partout dans le monde, à des fins commerciales ou autres, sur support microforme, papier, électronique et/ou autres formats.

L'auteur conserve la propriété du droit d'auteur et des droits moraux qui protège cette thèse. $\mathrm{Ni}$ la thèse ni des extraits substantiels de celle-ci ne doivent être imprimés ou autrement reproduits sans son autorisation.
In compliance with the Canadian Privacy Act some supporting forms may have been removed from this thesis.

While these forms may be included in the document page count, their removal does not represent any loss of content from the thesis.
Conformément à la loi canadienne sur la protection de la vie privée, quelques formulaires secondaires ont été enlevés de cette thèse.

Bien que ces formulaires aient inclus dans la pagination, il n'y aura aucun contenu manquant.

\section{Canadä}


The undersigned recommend to the Faculty of Graduate Studies and Research acceptance of the Dissertation

\title{
Aeroservoelasticity of articulated rotor hubs
}

\author{
Submitted by Derek Gransden \\ in partial fulfilment of the requirements for the degree of
}

Doctor of Philosophy

F. Nitzsche, Supervisor

M. Rose, Supervisor

B. Bornemann

M. Yaras, Department Chair

Carleton University 


\section{Abstract}

This thesis details the coupling of the rigid body parallel kinematics of an articulated rotor hub with a nonlinearly elastic rotor blade to provide vibration control to the fuselage and cockpit of the rotorcraft. A model rotor hub is examined, with distinction made between the aerodynamic load path and control load path, to complete the dynamic model. An examination of vibration suppression by replacing the standard pitch link in the control kinematic chain with an adaptive pitch link, suitable for vibration attenuation, is included. The mathematical basis for the elastic and rigid body elements of the simulation is described in detail. Computer simulations show the accuracy and validation of the constructed dynamic model, with a brief examination of control objectives suceeding. 
I dedicate this dissertation to my family and friends who supported me mentally, financially, and most importantly, emotionally. Without the love and understanding of my parents, Margaret and Andrew Gransden, this undertaking would certainly not have been possible.

I would like to extend this dedication to someone without whom I would not have my love of math, Mr. Arthur Wells. He was an excellent teacher in secondary school, and undoubtedly his efforts have produced many young minds in math, science, and engineering. 


\section{Acknowledgments}

I would like to thank Professor Nitzsche for giving me the opportunity to complete a doctorate. Without his support and advice, this project would not have been successful. I extend this thank you to Dr. Rose, whose counsel during my time at Carleton and the DLR was extremely helpful and informative. In addition, thank you for your support upon my arrival in Germany!

Also, I appreciate the input from all the professors at Carleton University, who have constantly provided help, insight, and comforting quips.

To my colleagues at the Technische Universität München, vielen dank! The knowl-

edge I gained from working with you was invaluable, and I will always remember how quickly you accepted me as one of your own.

I would like to acknowledge the Deutsches Zentrum für Luft- und Raumfahrt, for their financial contribution to my research, and for providing me an excellent opportunity to work among the helicopter vibration and control experts.

I wish to thank Ms. Elise Vist and Ms. Debbie Smith for their time and effort in editting my thesis.

And last, but never least, I thank all my family and friends; in particular the Culley family, who adopted me as an undergrad and have always treated me as one of their own.

Thank you all. 


\section{Table of Contents}

$\begin{array}{ll}\text { Abstract } & \text { ii }\end{array}$

Acknowledgments $\quad$ iv

Table of Contents $\quad$ v

List of Tables viii

List of Figures $\quad$ ix

Nomenclature $\quad$ xii

1 Introduction 1

1.1 Rotorcraft Introduction . . . . . . . . . . . . . . . 2

1.1.1 Kinematic Chains . . . . . . . . . . . . . . . . 4

1.1 .2 Swashplate .................... 7

1.1 .3 Pitch Link . . . . . . . . . . . . . . . . 9

1.1 .4 Rotor Blade . . . . . . . . . . . . . . . 10

1.1 .5 Aerodynamics . . . . . . . . . . . . . . . 10

1.1.6 Control Review . . . . . . . . . . . . . . . 12

1.1.7 SHARCS Project . . . . . . . . . . . . . 18

1.2 Literature Review . . . . . . . . . . . . . . . . . 20

1.2.1 Helicopter Blade Mechanics . . . . . . . . . . . . . 20 
1.2.2 Continuum Mechanics and Finite Element Analysis . . . . 23

1.2.3 Multibody Dynamics and Treatment of Constrained Dynamics 24

1.2.4 Aerodynamics . . . . . . . . . . . . . . . 28

1.2.5 Vibration Control . . . . . . . . . . . . . . . . 29

1.3 Objectives and Novelty of Research . . . . . . . . . . . 32

2 General Continuum Mechanics and Finite Element Development for Solid Structures $\quad 36$

2.1 Strain and Deformation _............... 37

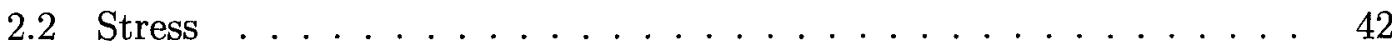

2.3 Governing Laws of Mechanics . . . . . . . . . . . . . . . . 44

2.3.1 Principle of Virtual Work in the Material Frame . . . . . . . 53

2.4 Finite Element Discretization . . . . . . . . . . 55

2.4.1 Generalized- $\alpha$ Method for Time Integration . . . . . . . . . 60

2.4 .2 Linearization and Iteration . . . . . . . . . . 62

2.5 Generalized- $\alpha$ Energy Momentum Method . . . . . . . . . . . . 64

3 Multibody Dynamics $\quad 69$

3.1 Modelling the Position and Orientation of Joints in Open Chains . . 70

3.1.1 Denavit-Hartenberg Representation of Forward Kinematics . . 73

3.2 Lagrangian Mechanics for a Multiple Degrees of Freedom Dynamic System . . . . . . . . . . . . . . . . . 75

3.3 Closed Chain Kinematics with Holonomic Constraints . . . . . . . . 81

3.4 Modelling the Rotor Hub Mechanics . . . . . . . . . . . . . 83

3.4.1 Rotor Articulation Holonomic Constraints . . . . . . . . . . . 89

4 Building the Aeroservoelastic Simulation 92

4.1 Aerodynamics . . . . . . . . . . . . . . . . . . . 92 
4.1.1 Momentum Theory . . . . . . . . . . . . . . . . . . 9 93

4.1 .2 Blade Element Theory . . . . . . . . . . . . . . . . 97

4.1.3 Blade Element Momentum Theory . . . . . . . . . . . 100

4.2 Control Theory and Application . . . . . . . . . . . . . . . 105

4.2.1 Overview of the Adaptive Pitch Link . . . . . . . . 105

4.2.2 Controlling Boundary Conditions . . . . . . . . . . . 107

4.3 Generalized- $\alpha$ Method for Index-3 Differential Algebraic Equations . 114

4.4 Elastodynamic Blade with Articulated Hub Boundary Conditions . . 117

4.4.1 Coupling Elastic Boundary Conditions with Hub Articulation 118

4.4.2 Coupling Elastic and Rigid Body Equations _... . . . 119

5 Numerical Experimentation $\quad 125$

5.1 Program Description . . . . . . . . . . . . . . . . . 125

5.2 Verification and Validation of Model Subcomponents . . . . . . . 128

5.2.1 Elastic Blade Foreshortening Effect . . . . . . . . . . . 129

5.2 .2 Four-bar Mechanism . . . . . . . . . . . . . . . 134

5.2.3 Aerodynamic Inflow Model Comparison to Experiment . . . 138

5.3 Verification of Complete Articulated Model . . . . . . . . . . . 140

5.3.1 Second-Order Convergence of Full Model . . . . . . . . . . 144

5.4 Validation of Complete Articulated Model . . . . . . . . . 147

5.5 Control Efficacy . . . . . . . . . . . . . . . . . . . 151

6 Conclusions and Future Work $\quad 154$

$\begin{array}{ll}\text { List of References } & 158\end{array}$ 


\section{List of Tables}

2.1 Dimensional maps between original and deformed configurations. . . . 46

3.1 Aerodynamic Chain . . . . . . . . . . . . . . . 84

3.2 Control Chain and APL DoF ................. 84

4.1 Coefficient of friction for sliding steel flat plate contacts. . . . . . . 113

5.1 Properties of the example elastic comparison beam. . . . . . . . . . 130

5.2 Percent difference verification of tip displacement in a cantilevered beam compared to 10 element model. . . . . . . . . . . . . . . 130

5.3 Time for static solution of multiple elements for cantilevered threedimensional beam. . . . . . . . . . . . . . . . . 131

5.4 Physical properties of the four-bar mechanism. . . . . . . . . . . . 134

5.5 DH parameterization of the four-bar mechanism. . . . . . . . . . 135

5.6 Period convergence of the 4-bar mechanism. . . . . . . . . . . 136

5.7 Crank-rocker mechanism analysis for velocity stabilization and Gen- $\alpha$ AB schemes. . . . . . . . . . . . . . . . . . 138

5.8 Properties of the aerodynamic inflow calculation and experiment. . . 139

5.9 Link properties for complete simulation verification. . . . . . . . . . 141

5.10 Hinge initial conditions, locked by user, based on trim hover conditions. 141

5.11 Link properties for complete simulation verification. . . . . . . . . 147

5.12 Hinge initial conditions, locked by user, based on trim hover conditions. 147

5.13 Link properties for complete simulation verification. . . . . . . . . 148 


\section{List of Figures}

1.1 Helicopter forward flight idealized airflow and lift. . . . . . . . . 2

1.2 Airflow perturbations during forward flight. . . . . . . . . . 3

1.3 Orientation convention for helicopter. . . . . . . . . . . . 4

1.4 Aerodynamic hinges for a typical rotor blade. . . . . . . . . . 5

1.5 A diagrammatical view of a generic rotor hub, including links. . . . . 7

1.6 Swashplate diagram with links, rotor blades, and control rods. . . . . 8

1.7 Active blade root control concepts. . . . . . . . . . . 13

1.8 Active blade twist concept. . . . . . . . . . . . . . . . 15

1.9 Embedded root smart spring concept. . . . . . . . . . 16

1.10 Axial smart spring concept. . . . . . . . . . . . . . . . 17

1.11 Base excited mass system. . . . . . . . . . . . . . . 18

1.12 SHARCS prototype blade with three independent control systems. . . 19

1.13 Miss-distance between rotor blade and vortex generated by previous blade. . . . . . . . . . . . . . . . . . 19

2.1 Undeformed and deformed configurations of a body described in coincidental frames of reference. . . . . . . . . . . . . 37

2.2 Stress elements on globular body. . . . . . . . . . . . . . . 42

2.3 2nd Piola-Kirchhoff stress tensor directions. . . . . . . . . . . 45

2.4 Undeformed and deformed configurations of a body. . . . . . . . . . . 48 
2.5 Graphical representation of finite discretization process and deforma-

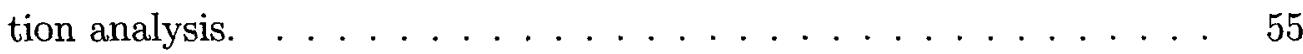

2.6 The isoparametric numbering system with some of the shape functions. 57

3.1 Position and orientation transformation between inertial and end frames. 70

3.2 Co-ordinate transformations in an open kinematic chain. . . . . . . 71

3.3 Three joints showing frames of reference and transformation parameters. 73

3.4 Diagram of a general closed chain kinematic mechanism. . . . . . . 81

3.5 Full hub articulation with coordinate frames listed. . . . . . . . 85

3.6 Full hub articulation with joint variables included. . . . . . . . . 86

3.7 The aerodynamic chain of the helicopter hub. . . . . . . . . 87

3.8 The control chain articulation of the helicopter hub. . . . . . . 88

4.1 Momentum theory flow diagram. . . . . . . . . . . . . 93

4.2 Momentum theory climb inflow diagram . . . . . . . . . . 95

4.3 Forward flight inflow theory diagram . . . . . . . . . . . . 96

4.4 Blade element theory flow and forces diagram . . . . . . . . . . . 98

4.5 Blade element momentum theory flow diagram . . . . . . . . . . 100

4.6 Adaptive pitch link attached to swashplate and blade root. . . . . . . 106

4.7 Finite element discretized elements constrained by aerodynamic chain 118

5.1 Flow-chart of the main program. . . . . . . . . . . 127

5.2 Foreshortening effect on a three-dimensional beam, compared at the

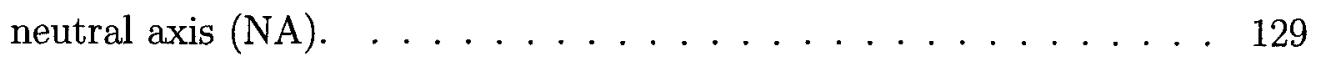

5.3 Distribution of displacement along the beam neutral axis from continuum statics approach with multiple elements in the $\mathrm{x}$-direction, one element in $\mathrm{y}$ - and $\mathrm{z}$-directions (except listed). . . . . . . . . 131

5.4 Distribution of displacement components along the beam with linear static $\left(^{*}\right)$, nonlinear static $(-)$ and foreshortening equation $(0) \ldots 132$ 
5.5 Beam displacement with nonlinear continuum mechanics with 10 elements. . . . . . . . . . . . . . . . . 133

5.6 A four bar mechanism with the open loop representation, cut at the end of the second link. . . . . . . . . . . . . . 134

5.7 The position solution to the 4-bar mechanism using the Gen- $\alpha$-AB method with different time-steps. . . . . . . . . . . . 135

5.8 The constraint forces using Gen- $\alpha$-AB methods combined. . . . . . 136

5.9 The position solution to the 4-bar mechanism using the velocity drift method (Blajer) and the Gen- $\alpha$-AB for constrained kinematics. . . . 137

5.10 Comparison of inflow between theory and NASA experiment. . . . . . 140

5.11 Simulated articulation in original position. . . . . . . . . . . . 143

5.12 Twist distribution of SHARCS rotor blade. . . . . . . . . . . 143

5.13 Displacements of flap, lag, and pitch link under trim hover aerodynamic load. . . . . . . . . . . . . . . . . 144

5.14 Resultant force vectors at the root of the helicopter blade. . . . . . 145

5.15 Resultant force vectors at the cut joint of the helicopter blade. . . . . 145

5.16 Convergence rates for orders of $\Delta t$ for the full articulated and elastic system. . . . . . . . . . . . . . . . . . 146

5.17 Experimentally measured pitch link forces and corresponding fast Fourier transform. . . . . . . . . . . . . . . . .

5.18 Experimentally measured pitch link forces, between $t=20.5 \mathrm{~s}$ and $t=20.75 s$

5.19 Simulated pitch link loads and corresponding Fourier transform. . . . 150

5.20 Control forces as a function of time and corresponding stiffness values at pitch link. . . . . . . . . . . . . . . . 152

5.21 Comparing the displacement with and without control implemented. . 153 


\title{
Nomenclature
}

\author{
ACF Actively controlled flap \\ ACT Actively controlled tip \\ APL Adaptive pitch link \\ DAE Differential algebraic equation \\ DH Denavit-Hartenberg \\ G- $\alpha \quad$ Generalized- $\alpha$ method \\ G- $\alpha$-AB Generalized- $\alpha$ method with acceleration modification \\ GEMM Generalized- $\alpha$ energy-momentum method \\ GEMMAB Generalized- $\alpha$ energy-momentum method with acceleration modification \\ GENUVP Generalized unsteady vortex panel \\ HHC Higher harmonic control \\ IBC Individual blade control \\ NA Neutral axis \\ ODE Ordinary differential equation \\ SHARCS Smart hybrid active rotor control system
}

A Undeformed area element

A Homogeneous transformation matrix

a Deformed area element

a Nodal acceleration

a Non-weighted acceleration

a Hand (end-effector) approach vector 


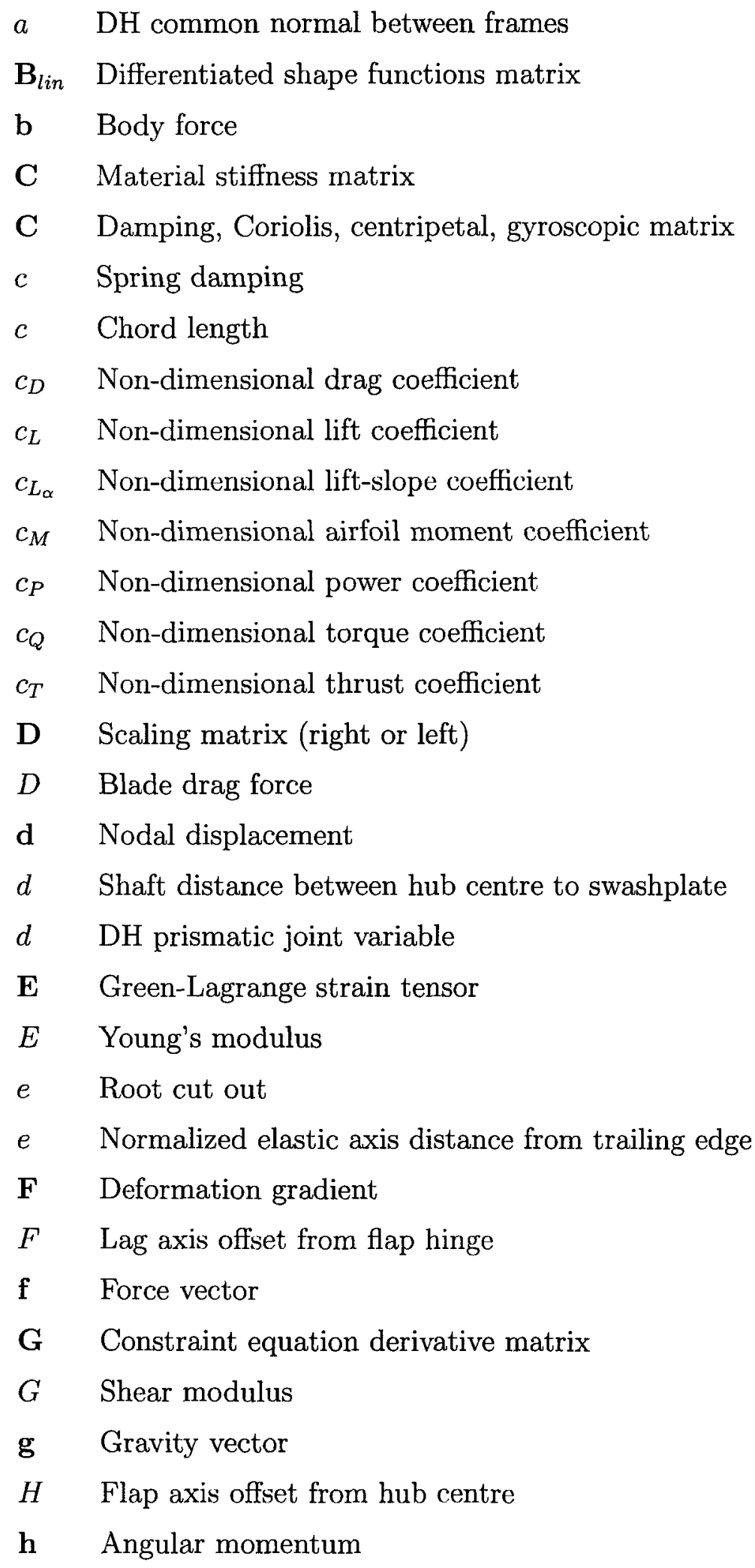


I Identity tensor or matrix

I Moments of inertia tensor

J Jacobian matrix

J Pseudo-inertia matrix

$J \quad$ Jacobian determinant

K Stiffness matrix

$K \quad$ Kinetic energy

$k \quad$ Spring stiffness

L Differential operator in matrix form

$L \quad$ Pitch axis offset from lag hinge

$L \quad$ Lagrangian

$L \quad$ Blade lift force

M Moment vector

M Mass matrix

$N(t) \quad$ Normal force from actuators

$N_{b} \quad$ Number of rotor blades

n Hand (end-effector) frame normal vector

$O$ Origin of coordinate frame

o Hand (end-effector) frame orienation vector

$P \quad$ Blade root offset from pitch hinge

$P \quad$ Pressure distribution

$P_{A} \quad$ Pitch arm length

$P_{H} \quad$ Pitch horn length

$P_{L} \quad$ Pitch link length

$P_{L} \quad$ Pitch link hinge variable

$P_{L_{2}} \quad$ Adapter mass hinge variable

$P_{O} \quad$ Distance between kinematic constraint coupling and rotor blade

$P_{s x} \quad$ Swashplate offset in x-direction

$P_{s y} \quad$ Swashplate offset in y-direction 
p Rigid body position vector from origin

Q Derivative matrix for rigid links

q Generalized joint variables

R Rotation matrix between coordinate frames

r Residual vector

r Position of a point within a coordinate frame

S Piola-Kirchhoff stress tensor

T Transformation matrix of chain end with respect to the origin

$T$ Thrust

$t$ Time

$t$ Blade (or element) thickness

$\mathbf{t}^{\hat{\mathbf{n}}}$ Traction tensor

U Matrices of link derivatives ( $1^{s t}$ and $2^{\text {nd }}$ order)

u Displacement vector

u Nodal coordinate system

$\mathbf{u} \quad$ Nodal displacement functions (trial functions)

$u^{*} \quad$ Strain energy density

$V \quad$ Undeformed volume element

$V \quad$ Potential energy

v Velocity (incl. nodal)

$v \quad$ Deformed volume element

$v_{\infty} \quad$ Air stream velocity

$W \quad$ Work

X Undeformed coordinate system reference

x Deformed coordinate system reference

x Global generalized coordinates

$x_{a} \quad$ Actuator mass displacement

$x_{b} \quad$ Bass mass (swashplate) displacement

$x_{c} \quad$ Controlled mass displacement 


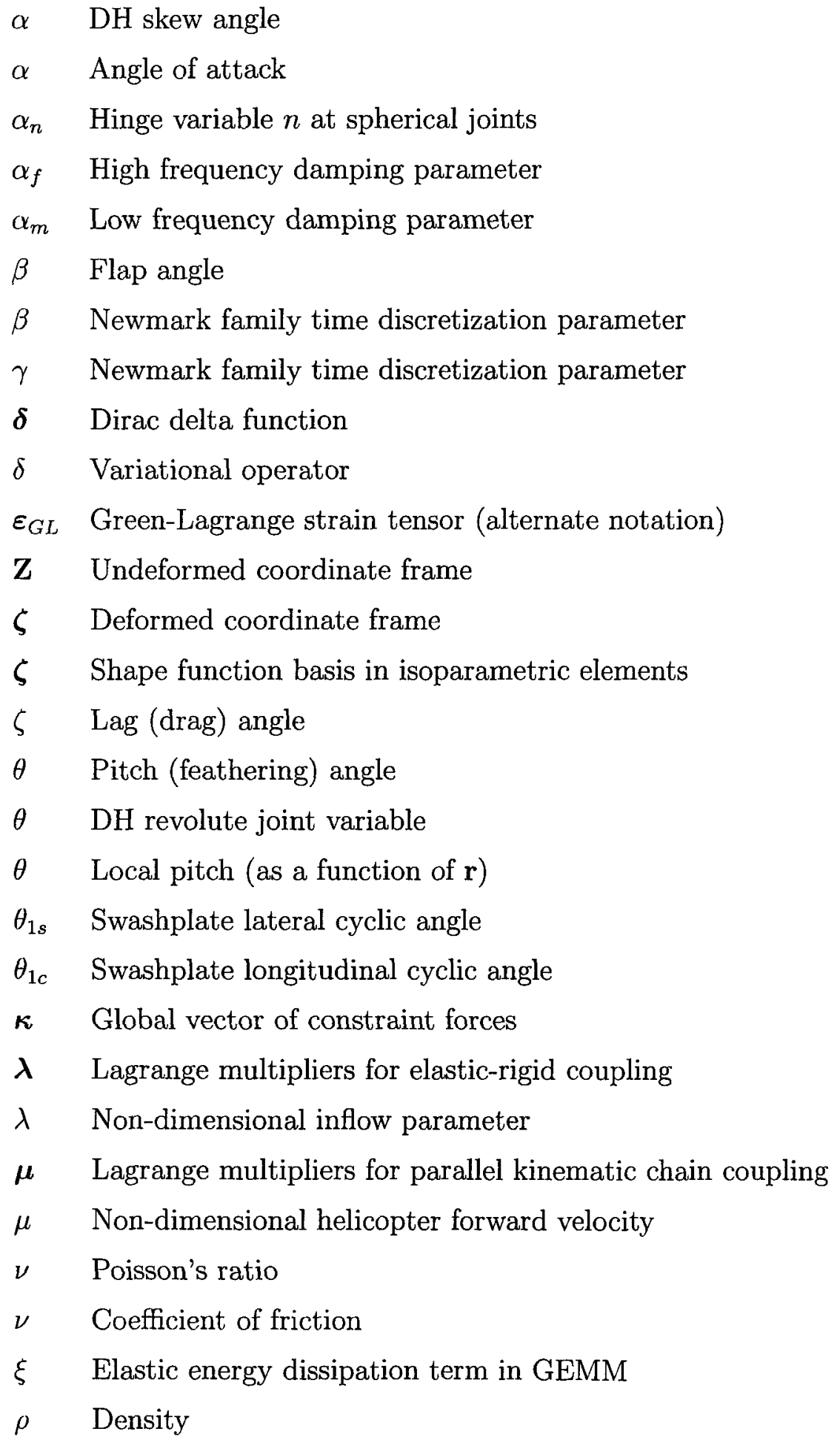




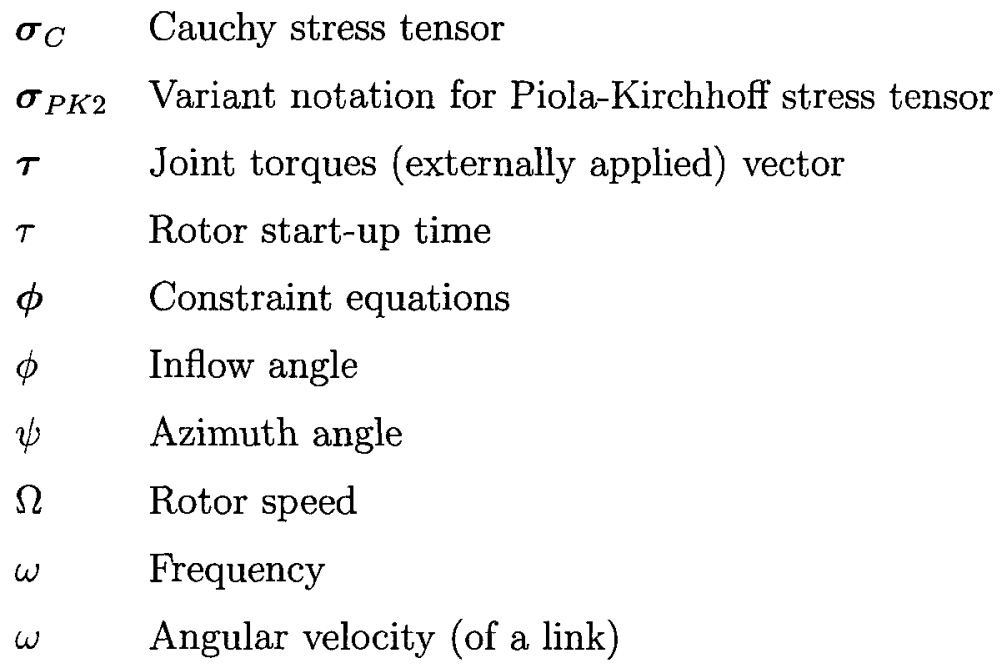




\section{Chapter 1}

\section{Introduction}

The introduction briefly covers the basics of the helicopter hub geometry, including how the helicopter blades are controlled by the pilot. The helicopter hub is grouped into two sets of hinges that provide all the necessary degrees of freedom for the aircraft to fly with high manoeuverability. Unfortunately, the hub also transmits vibration from the main rotor to the helicopter body, which decreases the aircraft efficiency and is detrimental to the pilot and cargo. Generally, vibration control research on helicopters seeks to reduce the airframe vibration by limiting the forces exciting the main rotor in two different ways: one tries to eliminate harmonic motions of the blades, the other tries to reduce the motions of individual blades.

After the review of the hub and control methods, a brief literature review is conducted. The literature review begins with an overview of beam models, which are far more common to rotorcraft blade dynamics. Beam models are not comprehensive enough to capture some torsional effects, so research into continuum mechanical models follows. It is necessary to compare solution techniques for rigid body articulation, so research on closed-chain kinematics multibody methods follows. A history of control methods, including vibration control ideas for helicopter main rotor vibration, finishes the literature review section. 
Having discussed previous attempts to improve vibration attenuation, the chapter concludes with an explanation of the innovation involved with this dissertation. The reasons for using an elastic blade model, the rigid body articulation, aerodynamics, and control strategies are explained in that section.

\section{$1.1 \quad$ Rotorcraft Introduction}

Rotorcraft are aerial vehicles that can manoeuvre at low or zero forward velocity. Because they are so mobile and can hover, rotorcraft are extremely useful in situations where small landing areas or maintaining a position over a ground station would be encountered. However, the functionality of helicopters is limited by the complexity of their designs, their low fuel efficiency, and the severe operating conditions that require harsh physiological exertion from the pilot. The pilot and cargo undergo large accelerations, which are due to the vibration transmitted to the fuselage that exceed comfortable limits [1] and cause further physiological demands on the pilot or damage the cargo [2]. This thesis details the reduction of main rotor vibration transmission to the fuselage via hub articulation by actively controlling the impedance of an adaptive pitch link.

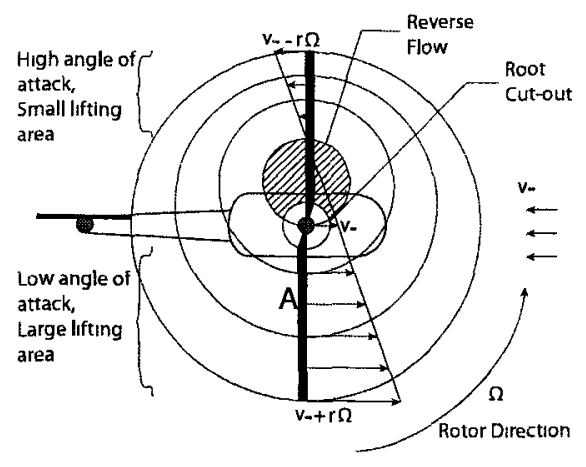

Figure 1.1: Helicopter forward flight idealized airflow and lift. 
First, it is necessary to examine the causes for rotor blade motion and helicopter vibration. Hovering flight, free from ground effects, is axisymmetric and there are no unbalanced moments. However, a helicopter in forward flight generates unbalanced aerodynamic moments due to the difference in lift in the rotor blades. Figure 1.1 shows a two-bladed helicopter in forward flight, such that the advancing blade at Position A and the retreating blade is in opposition. Due to the differences in airstream velocity, the advancing blade produces higher lift, which causes a moment that rotates the body of the helicopter fuselage, if there were no way to alleviate it. Similarly, other imbalances in the aerodynamic forces cause undesirable helicopter motion.

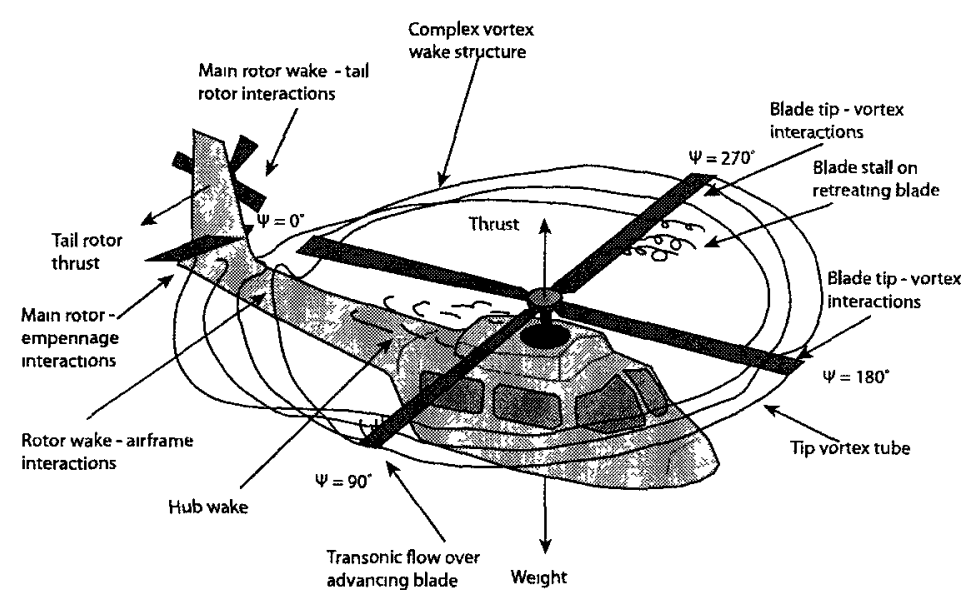

Figure 1.2: Airflow perturbations during forward flight. Adapted from [3].

In addition to the lift and moment imbalance, there are other fluid-structure interactions that cause perturbations in the aeroelastic system. Figure 1.2 shows the potential interactions during forward flight. Although the hub transmits the lift force to the helicopter, it also transmits vibratory forces to the fuselage and cabin. These mechanical vibrations arise in rotorcraft from three main sources: the engine, the higher harmonics from the main and tail rotors, and the low harmonics from the 
main rotor [4]. Of the vibration from these three sources, the most significant and the most detrimental to the aircraft and pilot are those resulting from the low harmonic vibrations from the main rotor [5].

Before commencing the analysis of rotor vibration suppression, it is convenient to describe some common rotor dynamics terminology. First, the body of the helicopter is typically described by its orientation. The three most common orientation descriptors are roll, pitch, and yaw. These three axes are shown in Figure 1.3. Note that it is not necessary for the centre of gravity to be colinear with the main hub shaft.

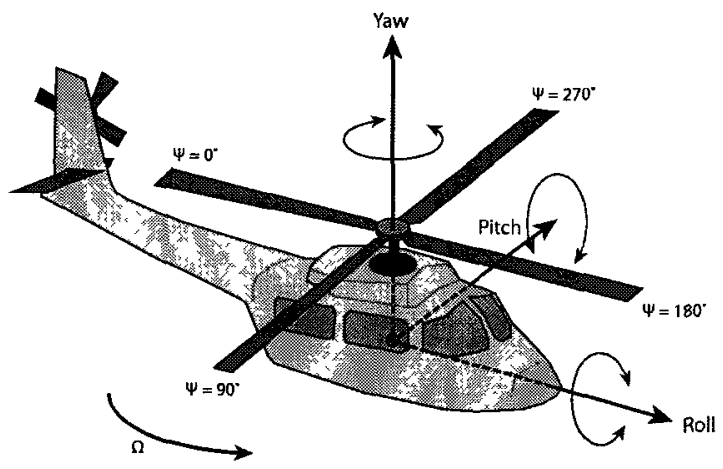

Figure 1.3: Orientation convention for helicopter.

\subsubsection{Kinematic Chains}

A kinematic chain is a group of links constrained by joints such that there is relative motion between the links. The kinematics of the helicopter hub splits into two chains: the 'aerodynamic' chain and the 'control' chain. These designations are used to distinguish the two open chains from each other by their function. Figure 1.4 shows the primary hinges of what is referred to in this thesis as the aerodynamic chain. The aerodynamic chain is so-called because its function is to relieve aerodynamic moments from causing undesirable revolute motion of the helicopter body. The control chain 


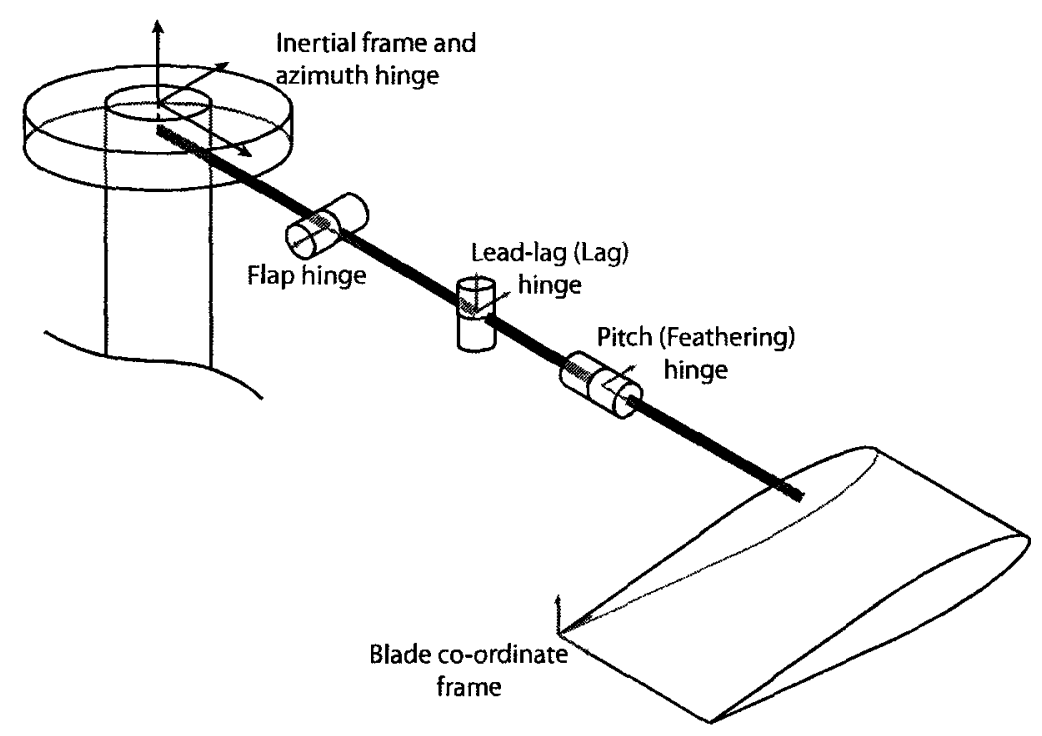

Figure 1.4: Aerodynamic hinges for a typical rotor blade.

is the mechanism by which the pilot can add input into the system. Typically, the aerodynamic chain is the mechanical system analysed in texts or papers that deal with hub articulation, while the control chain is often ignored.

The aerodynamic chain is the kinematic branch that links the helicopter hub to the blade root through the most direct path. It is the collection of joints associated with the aerodynamic characteristics of helicopter flight: the flapping, lagging, and feathering hinges. The order of the hinges does not affect their purpose; for example, the flap hinge can be inboard or outboard of the pitch hinge, but it will still prevent the unequal aerodynamic lift from causing rolling moments at the hub. The arrangement chosen in this work is as shown in Figure 1.4: the main hub, connected to the flapping hinge, which is often coincident with the lag hinge, and the pitch hinge farthest outbound. For the purposes of the research contained within this thesis, the inertial frame of reference of the helicopter is the centre of the hub, coincident with the axis of rotation.

The first hinge that succeeds the inertial point of reference is the azimuthal hinge, 
which describes the rotation about the hub. In North American helicopters, azimuthal rotations are counter-clockwise when looking at the helicopter from above, as in Figure 1.1. The helicopter power plant drives this hinge and rotates the remaining hub articulation and rotor blades about around it.

The flap hinge allows rigid body motion out of the hub plane; they were the first hinges proposed by early helicopter designers to prevent unbalanced lift forces from generating rolling moments that affect the cabin. However, a flapping blade experiences significant Coriolis forces in the hub plane, from the radial motion of the blade mass projected on the hub plane while simultaneously undergoing the azimuthal rotation of the hub. Therefore, an in-plane joint, called the lag, or drag hinge, is necessary to relieve the moments caused by those forces. Often this hinge is coincident with the flap hinge, which decouples the two joints, so they behave as a universal joint.

Each rotor blade must have a way to vary the angle of attack independently of one another. The flapping hinge changes the angle of attack by increasing or decreasing the incidence angle of the incoming airflow that the blade experiences. However, the pilot also needs to control the angle of attack to control the lift and thrust. To that end, the feathering, or pitch, hinge is used. The pitch hinge cannot be directly controlled in the rotating frame, so a control chain closes the kinematic loop, which transmits the pilot's control inputs.

The control chain is a bit more complicated, as Figure 1.5 indicates, with a comparison of the two chains together. There are, in total, twelve degrees of freedom in the control chain, including the adaptive pitch link. There is the azimuth angle, which affects both the aerodynamic and control chains. The next degree of freedom is the displacement down the shaft, which represents the commanded pitch angle. The rectilinear displacement of the swashplate along the shaft changes all rotor blade root pitch angles by the same degree. The next two degrees of freedom correspond to the 


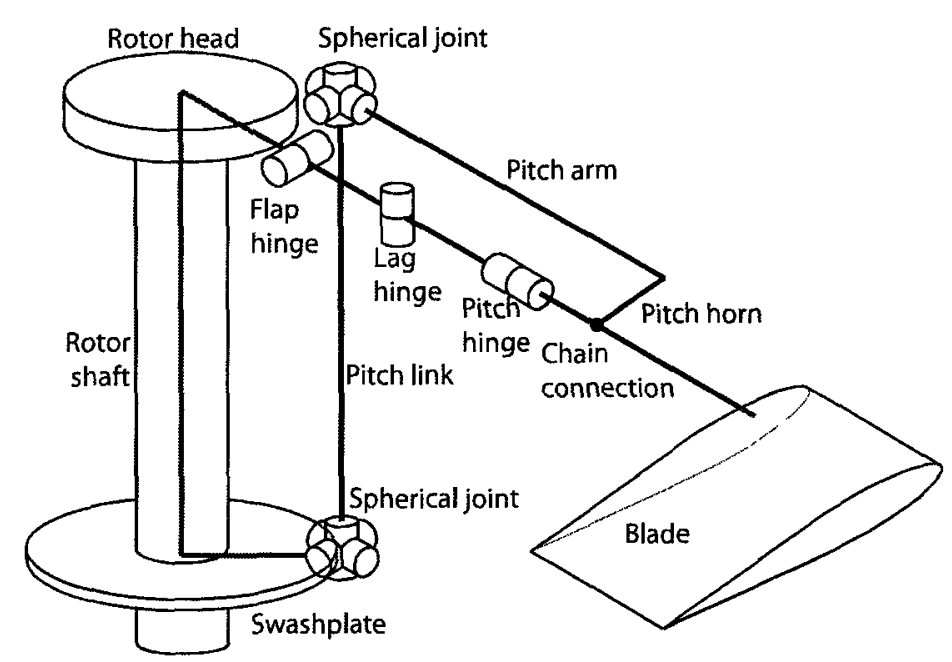

Figure 1.5: A diagrammatical view of a generic rotor hub, including links.

tilt of the swashplate, in the lateral and longitudinal directions. There is a spherical joint at the base of the pitch link, and one at the top, which accounts for three degrees of freedom each. (The bottom spherical joint is usually replaced by a universal joint; a spherical joint there is unnecessary and can cause singularities in the simulation solution. It is included as a spherical joint here for completeness.) Finally, the pitch link and the actuator mass each have a prismatic degree of freedom. Apart from the shaft, swashplate and pitch link, the control chain also subsists of the pitch arm and the pitch horn. The pilot indirectly controls the feathering angle of the blades at each azimuth position by altering the collective and cyclic tilt of the swashplate, and those changes are transmitted by the subsequent linkages.

\subsubsection{Swashplate}

The pilot controls the main rotor blades through the swashplate, which is a device that translates and tilts along the main rotor shaft according to the hydraulic commands from the cockpit. The position and orientation of the swashplate determine the pitch 
of the main rotor blades. The baseline pitch of the rotor blades, as controlled by the pilot, is called the collective, or collective pitch. The collective control affects all main rotor blades simultaneously and by the same degree. The tilt of the swashplate with respect to the shaft affects the angle of attack of each rotor blade differently, which is equivalent to cyclic control. The cyclic pitch varies with the azimuth angle, so each blade cycles through this pitch once per revolution, according to the orientation of the swashplate. In forward flight, cyclic pitch is the mechanism that allows the advancing blade to have a smaller angle of attack compared to the retreating blade. So, through the swashplate, the pilot controls three degrees of freedom: the collective pitch of all the blades, and the longitudinal and lateral cyclic degrees of freedom.

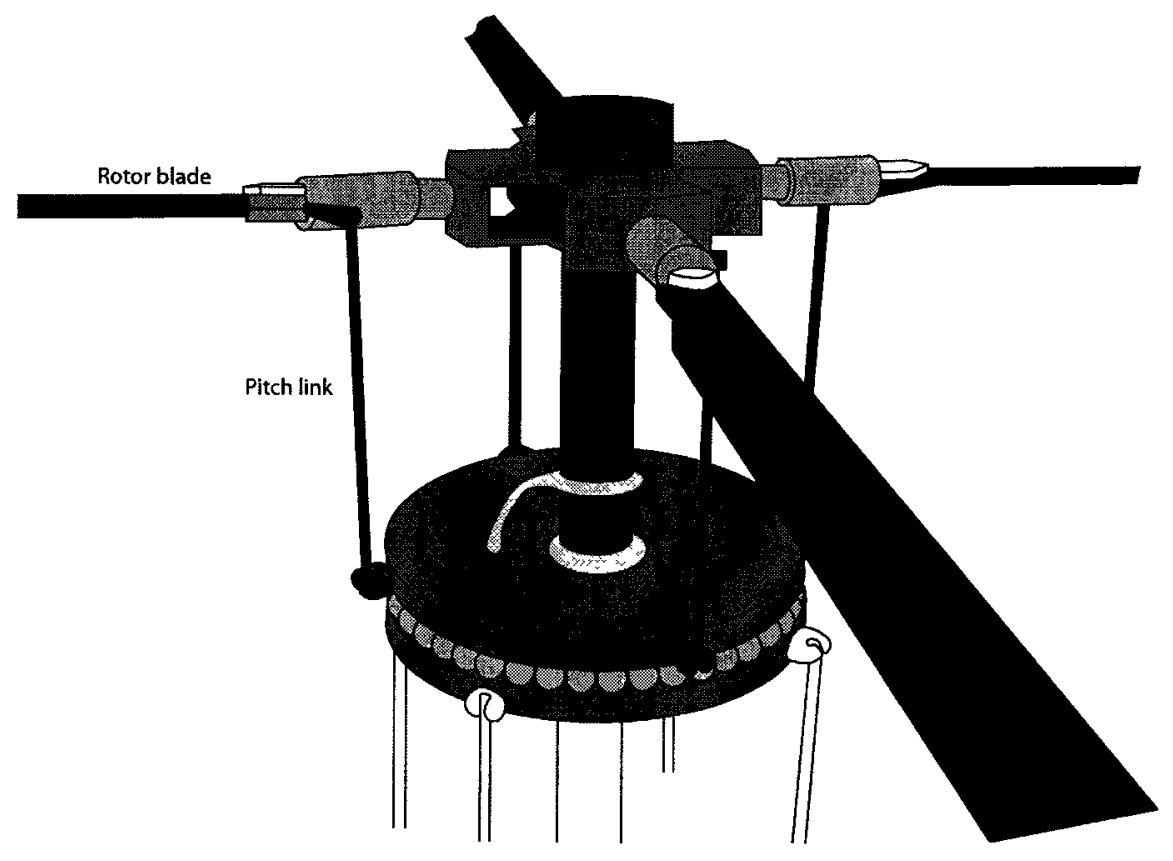

Figure 1.6: Swashplate diagram with links, rotor blades, and control rods.

Figure 1.6 shows a simplified swashplate with pitch link attachments and rotor blades. Theoretically, the swashplate is a pair of plates: one in the rotating frame, 
and one in the helicopter body-fixed frame. Both are centred on the main rotor shaft and free to translate vertically along the shaft as one unit. The pilot controls the hydraulic actuators that move the lower, non-rotating frame plate, while the upper plate rotates, on bearings housed between it and the lower plate, with the rotor head. The pilot can move these plates vertically and tilt them, in two independent directions, with hand controls in the cockpit. As mentioned, rectilinear motion of the swashplate induces a collective pitch at each blade root, and by differentially actuating the hydraulic supports of the swashplate, the pilot inclines it. The orientation of the swashplate determines the cyclic pitch of each of the blade roots, depending on the blade's location around the azimuth.

\subsubsection{Pitch Link}

The pitch link is the connection between the swashplate and the pitch horn and comes next in the articulated control chain. The pitch link functions as a device to map the bilateral inclination of the swashplate to sinusoidal motion projected on a cylindrical co-ordinate system. Within the rotating frame of reference, the incline of the swashplate becomes a sine wave with a period equal to one revolution around the hub. The forced motion delivered to the pitch hinge may not be exactly as a projection of the swashplate incline on a cylinder, because the pitch link is not fixed upright. Spherical joints permit pitch link rotation about its base upon the swashplate; however, the joints are typically damped and restricted, and the resultant motion determines the pitch at the blade root.

The pitch horn and pitch arm connect the pitch link to a suitable location on the aerodynamic chain azimuthally $\frac{\pi}{2} \mathrm{rad}$ ahead of the blade root. According to Bramwell [6], the blade control input must be $\frac{\pi}{2} \mathrm{rad}$ ahead of the blade's current position to affect its motion appropriately: gyroscopic precession causes an input force 
to be applied ahead of the desired location. Spherical joints at the tip of the pitch link nullify aerodynamic moments that might tend to bend the link itself. Because the joints are all revolute, moments are not transmitted directly to the helicopter fuselage. However, forces are carried to the helicopter frame, some of which are cancelled by forces from other blades, but unbalanced forces cause the undesirable helicopter vibration that limits aircraft performance and control.

\subsubsection{Rotor Blade}

Helicopter rotor blades are airfoils similar to those found on fixed-wing aircraft, except that they are rotated about the centre of the helicopter hub to produce propulsive forces from the airflow components parallel and out-of plane to the blade motion. Helicopter rotor blades tend to have much higher stationary compliance than fixedwing aircraft, because the centripetal stiffening effect from the rotor greatly increases the dynamic stiffness of the blade due to the internal tension caused by the angular acceleration.

A rotor blade typically connects to the hub through a bolted clamp that attaches to its inboard edge at the blade root. The rotor blade is much more aerodynamically efficient away from the blade root, but thrust is lost near the tip due to the air flow curling over the tip of the blade similar to fixed-wing aircraft. The benefit of controlling the blade root or articulation is two-fold: it is less aerodynamically obstructive and it is easier to replace than it is to change an entire rotor blade.

\subsubsection{Aerodynamics}

For simplified aerodynamics, there are three general theories: momentum theory, blade element theory, and blade element momentum theory. Momentum theory is 
the simplest approximation for rotorcraft aerodynamics, because it describes first order predictions of thrust (and drag), power, and torque. The Laws of Conservation of Mass, Momentum, and Energy are combined assuming quasi-steady, incompressible, inviscid, one dimensional flow to form momentum theory aerodynamics. Blade element theory (BET) is a more modern theory for helicopter aerodynamics, which uses a two-dimensional airfoil cross-sectional analysis to determine aerodynamic forces and moments. Sections are integrated over the length of the blade and the average over the rotor gives the global aerodynamic characteristics.

Blade element momentum theory (BEMT) is the combination of momentum theory with blade element theory. Of these three theories, BEMT is the closest approximation to actual rotor blade aerodynamics. Axial and hover cases are simple to analyse, since loading can be compared easily between the former aerodynamic models. However, forward flight is a greater challenge, since velocity is dependent on the blade azimuthal position, which creates a time-periodic non-uniform inflow pattern.

The non-trivial aspect of BEMT is choosing a description of air inflow velocity. Linear inflow models, such as Drees's or Glauert's, assume longitudinal flow with upwash at the leading edge and downwash on the trailing edge. These inflow models use coefficients to approximate a skew-angle that creates trochoidal airflow pattern. From experiments on trimmed rotors, inflow magnitude is greater towards the rear and slightly biased towards the retreating blade side. Mangler and Squire's non-linear inflow uses Euler equations to relate the pressure field across the actuator disc to the inflow; hence, it gives a better description of inflow over the rotor. Mangler and Squire's model is better near the edges of the rotor disc; it is a closer approximation to the more complex (and more accurate) vortex theory [3]. 


\subsubsection{Control Review}

The vibration must be either countered at the source or its transmissibility to the cabin and cockpit of the helicopter abated, because reducing the vibration directly requires large forces or deflections. Large forces or deflections are difficult to produce on lightweight aircraft, due to power and weight concerns. Typically, the amount of power required for directly affecting vibration prohibits its use on vehicles where keeping weight low is of supreme importance. However, there are numerous advantages to eliminating vibration through the hub, such as fewer mechanical fatigue problems, better pilot control, improved fuel efficiency, and a more stable cabin. Thus, a vibration reduction scheme must be robust and lightweight to justify its inclusion on an aerial vehicle.

The methods of vibration reduction can be grouped into one of three categories: passive, active, or semi-active damping. Passive damping involves stiffeners, dampers, and isolators that tend to effectively suppress the vibration, but only within a narrow frequency range [7]. Actively controlled structures can damp greater bandwidth; however, it is typically used to directly counter vibration and requires high power to produce large forces or large deflections [8]. Semi-active damping involves controlling the vibration using the impedance characteristics of the dynamic system. Semi-active controllers need less power than active controllers, and are advantageous over passive systems because they attenuate vibrations over a larger range of frequencies.

\section{High Harmonic Control or Individual Blade Control}

For main rotor vibration reduction there are generally two types of adaptive control: higher harmonic control (HHC) and individual blade control (IBC). An example of the non-rotating frame $\mathrm{HHC}$ and rotating frame root-IBC diagrams are shown in Figure 1.7. As can be seen, the higher harmonic control has an actuator below the 
swashplate, which operates in the inertial frame of reference attached to the helicopter fuselage. The booster amplifies pilot controls and passes them onto the HHC actuator, which filters the pilot signals and changes the tilt of the swashplate in conjunction with its control algorithms. Thus, all rotor blades are affected simultaneously by HHC. In the second case, the pilots signals control the swashplate directly and the individual blades are controlled based on the IBC controller algorithms separately. The IBC controller analyses the swashplate motion and uses its control algorithms to modify the pitch of each blade, in the rotating frame of reference, attached to the helicopter hub.

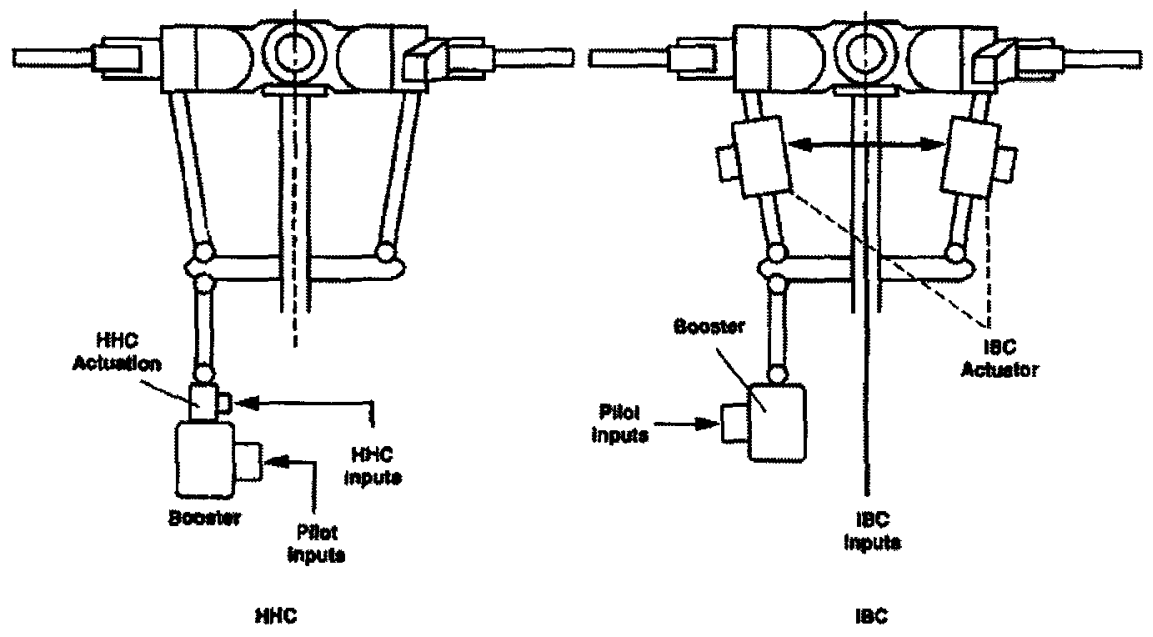

Figure 1.7: Active blade root control concepts [9].

Some studies show lower vibration levels and smoother ride accelerations in the helicopter using $\mathrm{HHC}$ (for example, [9]), but in the non-rotating frame of reference $\mathrm{HHC}$ can be difficult to implement. In HHC the rotor blades must perform identical motion, which restricts the frequencies of the higher harmonic blade pitch angle [4] (usually this is restricted to an $n_{b}-1, n_{b}$, or $n_{b}+1$ multiple of the rotor rotational speed, $\Omega$, where $n_{b}$ is the number of rotor blades). Other limitations with HHC implementation exist when dynamic coupling between the blade and the fuselage 
may not be neglected.

Individual blade control is a slightly different approach in that the actuators are placed directly on each blade, or its chain, and rotate with the hub. Studies show that using IBC can yield significant vibration reduction similar to $\mathrm{HHC}$, such as $\mathrm{Yu}$ et al. [9], or Ham [10], who describes some previous research on IBC and describes the advantages and disadvantages of each strategy. Experiments showing successful vibration reduction using IBC methods can be reviewed in References [11-13]. An individual blade control scheme depresses a broader range of vibration than a higher harmonic control scheme, approximately $2 \Omega$ to over $10 \Omega$ for a four-bladed rotor. It has higher control efficacy because it consists of separate subsystems designed to control the blade modes that have low damping ratios near the fundamental resonance frequency of the rotor [14].

\section{Active Twist Blades}

The IBC approach can also be subdivided into categories; most current research on the subject is on either active twist rotor blades or root actuation. The active twist rotor blades are usually composite fibre blades that have actuators between plies that contort the structure when electrical currents flow through them. Clusters of actuators on the rotor blade at its root, or as suggested in this dissertation, applied as a distinct, non-integrated, impedance device, achieve root boundary control.

As blade manufacturers used composite materials more readily, they realized local twists could be induced in the blades. These twists help control aeroelastic forces, as they could induce specific warping, bending, or further twisting that would distribute the stresses in all directions, or reduce and magnify aerodynamic loads on the wing. Previously this coupling was minimal and dependent on the materials used in the composite. A method of passively inducing blade twist to minimize vibration over a 
narrow band of frequencies using ply warping was examined in [15], which shows that passive vibration suppression is not the optimal solution for vibration attenuation in large bandwidths.

However, with the invention of smart materials, in particular anisotropic piezoelectric strain actuators, twisting a blade structure has become practical. Active twist rotor blades manufactured with composite material have piezoelectric actuators embedded within, those made with metallic alloys have surface mounted actuators, to modify the local twist of the blade appropriately.

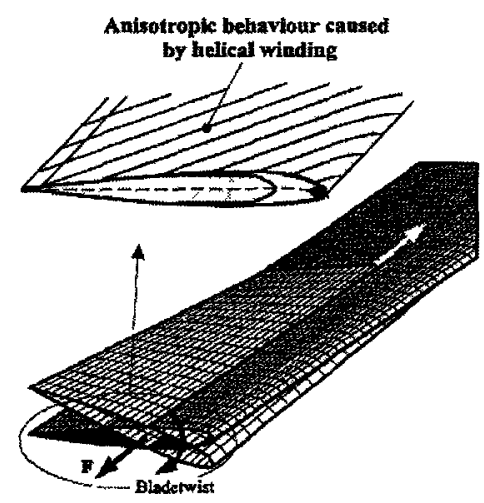

Figure 1.8: Active blade twist concept [16].

There is considerable research on active twist rotor (ATR) blades, as exemplified by Büter and Breitbach [16], and Cesnik et al [17]. These papers discuss active control methods with embedded piezoelectric actuators in conjunction with directional fibre winding, as shown in Figure 1.8. The ATR blades have driving actuators that result in local deformations, which in turn influence the blade twist distribution. By locally altering the twist distribution of the airfoil, an effective controller temporarily changes the lift, twist, and drag properties of the rotor blade. Although this type of vibration control has been successfully introduced, it generally requires more power to develop full control than root methods. This type of control constantly works against the 
aerodynamic forces applied to the blade, and therefore, significant power consumption is typically associated with this active control.

\section{Root Control}

Root vibration control is also a matter of current research and shows promise mitigating vibratory loads generated from unsteady aerodynamics of the rotor blade. There are many different methods of achieving rotor blade root control, including incorporating smart materials within the blade, fastening actuators to the surface of the blade, or replacing the pitch link with a semi-active muffler.

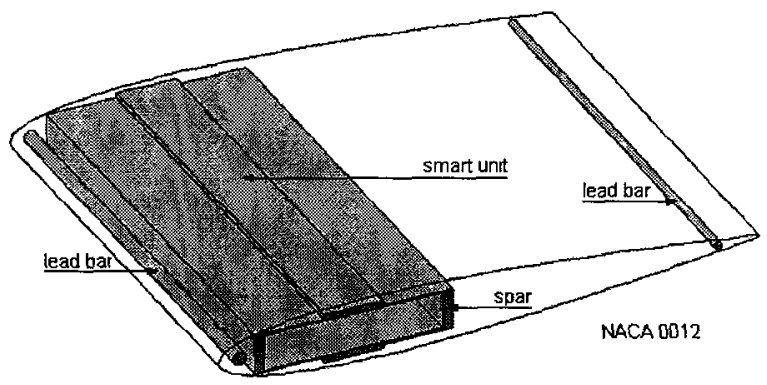

Figure 1.9: Embedded root smart spring concept [18].

A system with embedded actuators in a composite rotor blade is shown in Figure 1.9. Systems with embedded or fastened actuators achieve control by creating an internal moment within the blade that changes the load distribution along the blade. This is similar to the active twist concept; however, the load distribution is changed at the root of the blade, which affects the strain energy density throughout the blade that changes its vibration characteristics. Similar to the active twist blades, root control of helicopter blades using embedded or surface fastened smart materials also causes discontinuities, and requires high power consumption.

Controlling the blade at the linkage, as shown in Figure 1.7, obviates concerns pertaining to local discontinuities. Instead of creating unwanted shear and moment 
effects within the blade, the root control device controls the motion of the linkage system and thus the blade motion. This system has the added benefit of altering the boundary conditions, which is a well-known method used to change the vibration characteristics of a mechanical system.
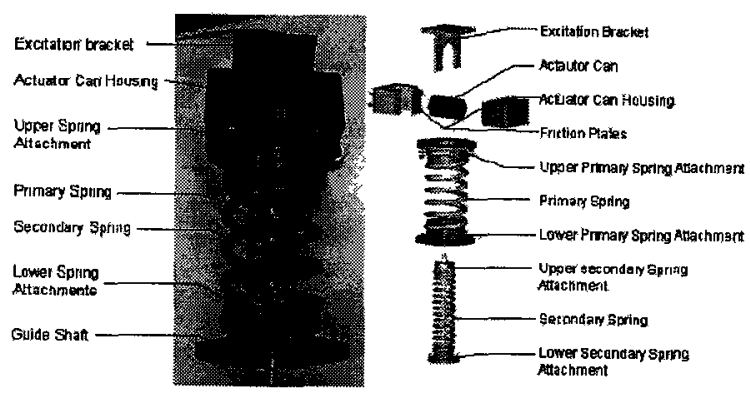

Figure 1.10: Axial smart spring concept [8].

Harold [8] describes an impedance control device, called a 'smart spring', that affects the mass, damping, and stiffness characteristics of the system to which it is added. A smart spring device employs piezoelectric elements in a stack as an actuator can, as shown in Figure 1.10. Comparing Figure 1.10 to Figure 1.11, the active material mass is equivalent to the actuator can and the actuator can housing; $k_{1}$ is the primary spring; $k_{2}$ is the secondary spring; the controlled mass corresponds to the rotor blade and the base mass is the swashplate, neither of which are shown.

When inactive, the actuator block slides freely in a slot that is attached to the controlled structure, as shown in Figure 1.11. However, when the actuator is activated it contacts the side-wall surface of the slot and causes friction. Heat from friction dissipates energy out of the system the smart spring is designed to control. In this way the smart spring can control the impedance of a mechanical system. 


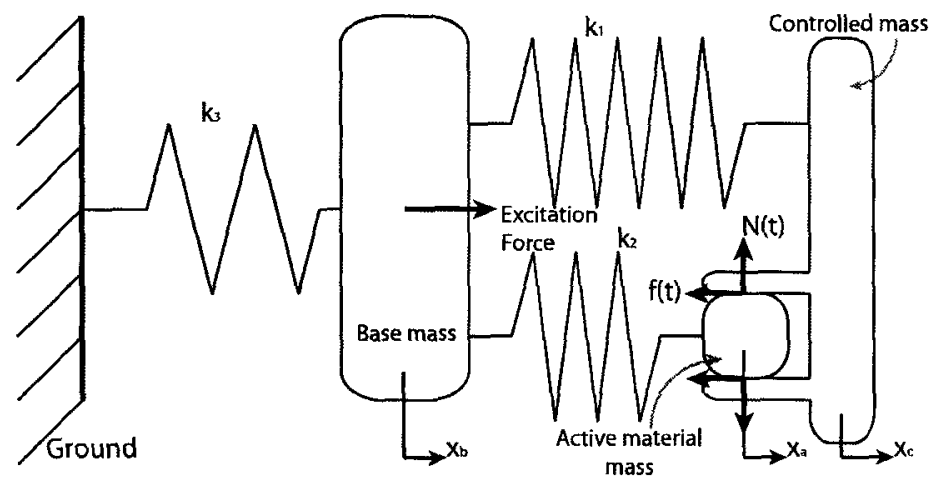

Figure 1.11: Base excited mass system [8].

\subsubsection{SHARCS Project}

The Smart Hybrid Active Rotor Control System (SHARCS) project is the total rotor blade control research proposal, of which this thesis is part, introduced at Carleton University, which can be further reviewed online [19]. Because control strategies typically cannot reduce both rotor noise and vibration simultaneously, this project aims to supplement rotor blades with three subsystems that are fit onto an existing rotor blade. These three subsystems are independently controlled, this creates a control system to be able to coincidentally affect noise and vibration reduction. The subsystems are an Actively Controlled Tip (ACT), an Actively Controlled Flap (ACF), and an Adaptive Pitch Link (APL). The sketch of these three devices on the rotor blade is shown in Figure 1.12.

One of the significant sources of noise and vibration is caused by blade-vortex interaction (see Figure 1.2). There are two ways to reduce the noise emanating from that interaction: reduce the strength of the vortex or increase the miss distance of the blade, see Figure 1.13. The actively controlled tip is an anhedral flap device that achieves noise reduction by changing the blade's tip geometry. Due to its flapping motion, it maximizes the vortex miss distance with the preceding and succeeding 


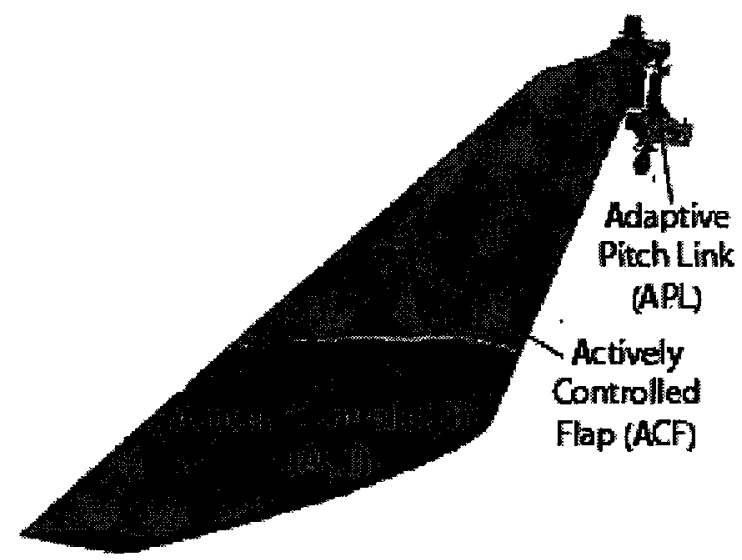

Figure 1.12: SHARCS prototype blade with three independent control systems [20].

blades. For further information, prototype details are available in Ref. [21].

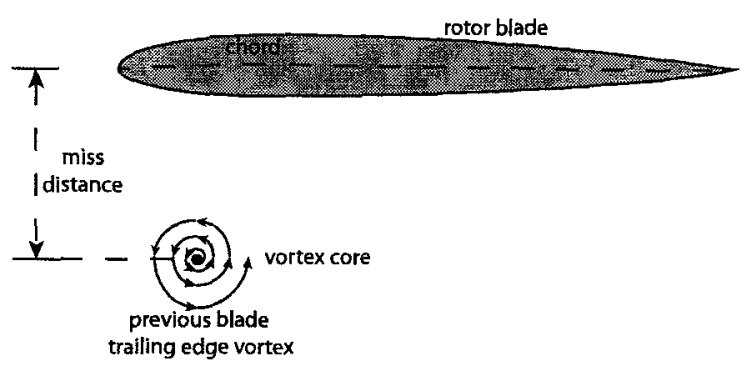

Figure 1.13: Miss-distance between rotor blade and vortex generated by previous blade.

An actively controlled flap is fit to the aerodynamically efficient section of the rotor blade to reduce rotor blade vibrations. At moderate advance ratios, vibration becomes a limiting factor in helicopter performance, so the ACF prevents dynamic stall and diminishes hub vibration loading by oscillating the blade angle of attack with the azimuth angle. At higher advance ratios, dynamic stall cannot be avoided, but it can be lessened by reducing the local angle of attack. More information can be found in References [22,23] and the thesis by Ülker [24].

The goal of the APL is to replace the nominal pitch link in the helicopter control 
chain and reduce the transmission of vibratory loads to the fuselage via the hub. Smart material activation engages a block housed within the adaptive pitch link system. Engaging the mass causes friction forces that convert the kinetic energy of the combined rotor blade-block mass system into thermal energy. In addition, there is a momentum transfer between masses, and the combination of these effects reduces the vibration transmissibility of the hub. An initial study on the dynamics of this system can be found in Gransden [25] or, with aerodynamics, in Nitzsche and Oxley [26].

\subsection{Literature Review}

Due to the nature of the thesis, which involves the combination of four distinct topics (blade mechanics, multibody articulation, aerodynamics, and control), each subject is reviewed separately. There are many papers that overlap specific subject areas, so these works are included where they were most applicable. What follows is a semi-chronological overview of how this research developed.

\subsubsection{Helicopter Blade Mechanics}

Early investigations into helicopter blade dynamics focussed on cantilevered blades attached to a rotating shaft. Perhaps the first investigation using linear analysis was that of Houbolt and Brooks [27]. They performed a first order analysis using a Newtonian approach. Their research was superseded by Hodges and Dowell [28], which included non-linear terms of a slender, homogeneous, isotropic blade.

Considerable research went into the modal analysis and stability of vibration of cantilevered beams, after these aforementioned works. Two books that use beam models in their description of helicopter blade mechanics are Bramwell's Helicopter 
Dynamics [6] and Johnson's Helicopter Theory [29], and they are still regarded as excellent initial sources. These books both contain considerable detail on rigid body motions of the hub, the elastodynamics of the blade, and flight aerodynamics.

Yokoyama [30] researched the effects of shear deformation on the motion of the blade. It was discovered that shear effects, which were previously unresearched, were more pronounced in a slowly rotating blade than a non-rotating beam. This is one disadvantage of Euler beam models; however, the effects were less severe as the rotational speed increased.

Borri [31] introduced a kinematic chain (an open chain) into the helicopter time domain research. His paper seemed to be one of the first attempts to realize a coupled response between a mechanism and a rigid blade model. This research was conducted with finite element analysis, and included some simple aerodynamics in the formulation.

Al-Bedoor and Khulief [32] investigated elastic beam models and the vibration analysis including prismatic and revolute joints. This was done in the frequency domain, using cantilevered beam shapes. Dynamic models with coupled responses became more important, so Turnour and Celi [33] examined the coupling between the entire helicopter and the blade motions. They included a tilting shaft and used linearized aerodynamic load models. Al-Bedoor [34] further examined this aspect of rotor deflections, shaft torsion, and bending modes. This work was one of the papers that split the motion into elastic body and rigid body motions. Finite element analysis was also performed in this paper.

The articulation of the hub and the coupling effects of the rigid motion with the elastic blade became increasingly important to accurate helicopter blade simulation. Yoo and Shin [35] published results on the effects of the flap-lag coupling, using some gyroscopic terms neglected in Hodges and Dowell. Chung and Yoo [36] published a 
paper with non-linear finite elements, but used a linear beam model and only studied the effects of the flap-lag coupling.

A paper by Zheng, Ren, and Cheng [37] combined the elastic blade deformations of Hodges and Dowell with the aerodynamics of Hodges and Ormiston [38], and included a detailed examination of the aeroelastic hinges connecting the hub to the blade root. They included the hinges commonly found on most helicopters, the flap, lag, and pitch, and coupled the entire hub system to an Euler-Bernoulli beam system representing the helicopter cabin. Instead of using Lagrange multipliers, they iterated between solutions of the hub system and fuselage beam.

Cesnik et al. [17] worked on the variational asymptotic method; which is a onedimensional plus two-dimensional $(1 \mathrm{D}+2 \mathrm{D})$ analysis. This method emulated the effects of a three-dimensional analysis by decomposing the physical model into two groups: a cross-section and a beam model. The advantages of this method were that it solved the equations of motion quickly and it was accurate for most three-dimensional effects. Their paper combined the experimental research done on a hover flight case with an articulated rotor. They showed, experimentally, that the individual blade control concept was effective at reducing the severity of blade vibration. Their research was conducted to show the effectiveness of active twist rotors, which in general requires higher loading, higher blade forces, and have higher voltage requirements.

Subsequent studies using finite element analysis have shown that a threedimensional analysis can capture effects unnoticed by $(1 \mathrm{D}+2 \mathrm{D})$ methods. Truong [39] showed, with an element model of 10000 nodes, that there were high-speed torsion effects that were more accurately predicted by a finite element model. The program used in that paper was designed for mid-range workstations, much like the one described by this thesis. However, there were no aerodynamic effects, nor were there hinges applied to the mathematical model, which is included in this dissertation. 
Das, Madenci, and Straub [40] published a paper similar to Ref. [39] in that they used finite element analysis to capture aeroelastic effects of flexible rotor blades. Shell elements with transverse shear were used in their paper, which included a quasisteady aerodynamic analysis based on lifting line theory. They agreed that $(1 \mathrm{D}+$ 2D) models are convenient in that those methods contain fewer elements, but that full models capture three-dimensional effects around the cross-section more realistically. They used shell elements specifically to capture shell-like deformations similar to those experienced by flexbeams. Das et al. noted that they have approximate aerodynamics, and they perceived that as fluid mechanics becomes more refined, so should the solid structural mechanics. Therefore, the research described by this thesis contains a three-dimensional continuum mechanical model, because of its ease to construct, and to capture torsional effects that may be important for the control, given that it is based on pitch control.

\subsubsection{Continuum Mechanics and Finite Element Analysis}

Well written initial research on continuum mechanics and tensor calculus include Bonet and Wood [41], Haddad [42], and Mura and Koya [43]. Each of these references began with basic continuum mechanics and developed the material relationships with tensor calculus.

Crisfield $[44,45]$ concisely meshed the combination of continuum mechanics and finite element analysis into a couple of comprehensive texts. Bornemann and Wall [46] (or Bonet and Wood) wrote an easy-to-understand linear finite element text and continued their work with a non-linear version [47], which also delved into a more detailed analysis of parameterization methods, such as Newmark's method and the Generalized- $\alpha$ method. These also detailed how to convert the tensor calculus notation into Voigt matrix and vector mathematics for computer manipulation. 
Kuhl and Crisfield [48] published a paper describing various energy-conserving and decaying methods pertaining to structural dynamics. This paper compared Newmark's Method, classical $\alpha$-methods, the Energy-Momentum Method, and Constraint Energy (and Momentum) Methods. It resulted in a Generalized- $\alpha$ Energy-Momentum Method, which formed the basis of elastodynamics in this thesis. This method combined the energy and momentum conserving properties of the Energy-Momentum Method with the numerical stability and controllable numerical dissipation of the $\alpha$-methods.

\subsubsection{Multibody Dynamics and Treatment of Constrained Dynamics}

The multibody dynamics described in this thesis are divided into two aspects: computational dynamics and optimized constraints. The computational dynamics research for this thesis used the approach found in many robotics textbooks. Two primary texts used in this research were Introduction to Robotics: Analysis, Systems, and Applications [49] and Modelling and Control of Robot Manipulators [50]. Both of these works provided details on open and closed kinematic chain successive reference frame analysis. References [51-54] included computational dynamics with some constrained analysis. Typically, these books included constraints as forces applied externally to a set of elements. Reddy and Rasmussen [55] was particularly useful in its inclusion of variational methods. The Lagrange multipliers they use were treated as internal variational forces that couple multiple bodies.

There are a few papers specific to helicopter multibody dynamics. Of particular interest were those that incorporate the blade mechanics with the articulation, such as Agrawal [56], which explained clearly a method for analysing the multibody blade 
dynamics with a full helicopter model. In that paper, Lagrange multipliers were used to showcase the coupling between the hub and a blade system. This paper also explained the difficulties with existing models. CAMRAD II [57] and DYMORE [58] are current helicopter structural models that are expensive, difficult to expand, difficult to use and modify, and for experimental purposes, restrictive in their generality. These structural models also used beam elements in a $1 \mathrm{D}+2 \mathrm{D}$ solver.

Although it used modal analysis, the paper by Rosen, Loewey, and Mathew [59] determined a method to include hinges at the root of the helicopter blade. This work had only the aerodynamic hinges, but it also showed the effect of springs and hinges on the blade mode shapes. Continuing with a beam model built from their previous paper, Loewy et al. [60] analysed root retention variations, which showed the modal coupling between hinges, and the stability provided therein.

Bauchau and Kang [61] published a paper in which they modelled the combined elastic and rigid body motion, as opposed to solving the body motions separately. They used a pitch link to model the motion; however, they discuss the addition of constraints and the destabilizing effect adding a rigid infinite stiffness has on a system.

Bauchau [62] examined the matrix condition stiffness of a system of equations due to the effects of Lagrange multiplier constraints. He noted that energy preserving schemes have difficulty with high frequency numerical dissipation, which can cause convergence problems. It was suggested that energy decaying methods provide unconditional stability, especially in the presence of constraints. (Laulusa and Bauchau [63] review these methods in a more recent paper.)

Again, Bauchau, with Bottasso and Nikishkov [64], published a history of blade models and multibody methods. They used a model based on the variational asymptotic method, and include joints, modularity, and Lagrange multipliers for (matrix) sparsity. They outline the utility of various methods: $1 \mathrm{D}+2 \mathrm{D}$ solvers for quick, 
accurate results; shell methods for flexbeams and bearingless rotors; and 3D solid models for a realistic beam. One of the other distinguishing features of this paper is that it suggested the importance of the control chain on the overall motion of the helicopter blades.

\section{Closed Chain Multibody Dynamics}

Using a Newton-Euler analysis with Denavit-Hartenberg formulation for the frames of reference, Luh and Zheng [65] mimicked the analysis of an open tree-structured robotic system with a closed kinematic chain mechanism. They described a virtual cut to a link forming part of the closed-loop chain, which was replaced by holonomic constraints. Then they describe the methodology to solve the open-loop system. In a later paper, Zheng and Luh [66] include an analysis for the velocity constraints, which were used instead of the position and orientation constraints for clarity and ease-ofuse. The drawback with velocity constraints, however, was that they can be nonholonomic, and would have to be treated separately. This led to a two-field problem of positions and velocities, and their constraints, which increased the complexity of the solution and increases the computational cost.

Nearly the same time as Ref [65], Gear, Leimkuhler, and Gupta [67] showcased a method to solve two-field problems. Their work showed an easy way to reduce the index of differential algebraic equations (DAEs) generated by constrained mechanics. This reduction makes the DAEs easier to solve, but the increased computational cost remains.

Another drawback in the two- and three-field analysis is the phenomenon of drift in the loop closure, because as time elapses, the closed-loop system appears to generate 'gaps' in the solution at the virtually cut joint. Often the recommended procedure was to reduce the system to a minimal set of co-ordinates [68-70]. However, the argument 
against using a minimal set of state variables is that they require complicated symbolic manipulations that are unwieldy and not guaranteed to have a unique solution.

Blajer $[71,72]$ published two papers to uncover a filtering method that reduced the constraint errors. State variables were corrected geometrically to eliminate constraint violations, the 'gaps', at each integration step. Instead of using a minimal set, Blajer solved a dependent set of state variables with the constraints in a differential algebraic equation. Kövecses, Piedboeuf, and Lange [73] improved filtering techniques based upon Blajer's work by introducing a filtering method that splits the joint variables into variables that satisfy the motion of the system, and variables that are admissible with the constraints. Unfortunately, filtering techniques cost even more computational time, and required more iterations in a non-linear analysis.

Calculating joint torques posed another quirk of closed chain kinematics: openchain analysis normally involved a robot Jacobian relating the torques to the input forces. In the case of closed chain kinematics, the torques were not easily calculated because of the static indeterminacy. However, an analysis by Śmiarowski and Anderson [74] showed that a Jacobian could be obtained by any path and that it could be related to all joints via the constraints.

A paper published by Negrut et al. [75] established a method of manipulating the index-3 DAE problem without resorting to a two-field problem. They used a Newmark family method, although not generalized- $\alpha$, and a predictor-corrector to tackle the high-order differential algebraic equation. Lunk and Simeon [76] reiterate their findings, and compared methods with position and velocity stabilization (twofield problems) with the index-3 methods. They, along with Khude et al [77], showed second-order convergence for regular index-2 problems.

Recently, Arnold and Brüls [78] showed that a generalized- $\alpha$ solution to an index3 differential algebraic equation was possible by using an alternative description of 
the acceleration, instead of relying on a weighted formulation of the residual equation. Their formulation used only position constraints and still showed second-order accuracy and quadratic convergence even with Lagrange multipliers.

\subsubsection{Aerodynamics}

SMARTROTOR is a program that analyses helicopter aeroelastic interaction. There are three main sections to SMARTROTOR: an aeroacoustics package, an aerodynamics package, and a structural package. The aeroacoustics package was designed by Opoku [79] to determine the noise generated by the helicopter in various flight configurations. However, it is not a major component of this research.

The SMARTROTOR aerodynamic component (GENeral Unsteady Vortex Panel, GENUVP), was developed at the National Technical University of Athens (NTUA) and will be combined with the structural solver described by this thesis, as part of the SHARCS project. GENUVP uses the Helmholtz theorem, which allows the decomposition of an incompressible inviscid flow-field into its rotational and irrotational components: the uniform external velocity, the velocity induced by the solid panels, and the velocity contribution from the rotor wake. GENUVP combines a vortex particle method to represent the rotor free wake as a cloud of particles and an indirect panel method to represent the solid bodies. Using a smoothed approximation to the Biot-Savart Law [80], the velocity induced by the cloud of vortex particles is computed.

In GENUVP, tip and trailing edge wake strips are emitted at each time step to form a so-called near-wake. These are represented by dipoles whose intensities are matched with the intensity of the emitting elements at the lifting surfaces to enforce the Kutta condition at the tip and trailing edges. The near and far wakes are coupled such that at each time step, a vortex particle is produced by integrating the vorticity 
of each near-wake element. Next, the far wake is formed by these particles and is convected and deformed in two steps. First, the particles are convected according to the local velocity, and then they are deformed to take into account vortex stretching using a particle strength exchange method. For a complete description of this formulation, see Voutsinas et al [81-84]. GENUVP is capable of simulating a complete helicopter rotor configuration and able to capture the blade-vortex interaction effects, important in the prediction of helicopter rotor aeroacoustics.

As the focus of this dissertation is computational structural dynamics, the aerodynamics used in this dissertation are based upon the blade element momentum theory model. Leishman [3] is the primary source for lift, drag, and twisting moment aerodynamics. Comparable sources include Bramwell [6] or Johnson [29], although their descriptions of aerodynamic loads were based on different definitions of initial positions. Instead of constant or linear inflow theory, however, Mangler and Squire's [85] non-linear inflow aerodynamic model was used. This inflow theory more accurately described the air inflow through the rotor actuator disc as compared with NASA experimental results [86-88].

\subsubsection{Vibration Control}

Due to the nature of the SHARCS project, individual blade control and root semiactive control devices will be examined. Initial investigations on individual beam vibration were performed by Hodges $[89,90]$. These examinations of cantilevered beam models included off-axis rotational formulae and natural frequency calculations. Beam vibration and control research continued with works similar to Biswas and Klafter [91] and Diken [92]. These works had no hinge mechanics, and looked at feedback control of a fixed-free rotating beam, without aerodynamics.

More robotic vibration damping was investigated by Dadfarnia et al. [93] and Choi 
and Han [94]. These works examined robotically controlled systems, with piezoelectric patches applied to the robotic arm. Neither of these works included aerodynamics, but showed tip deflection suppression. Na, Librescu, and Shim [95] modelled a closer approximation to a rotorcraft blade. They also used bonded surface piezoelectric patches over the spar of the beam to achieve out-of-plane actuation (flap) and used feedback control to control the flap and lag response of the beam tip. However, they did not include hinges or aerodynamics.

Aeroelastic analyses of rotor vibration control can be found in many sources, such as Nitzsche and Breitbach $[14,96,97]$. Their first paper detailed a smart structures technology as embedded piezoelectric patches in an individual blade control algorithm. Although the equations of motion are linearized, this paper includes forward flight aerodynamics and examined the modal properties of the blade. Nitzsche and Breitbach [97] continued their research with high harmonic vibration of hinged helicopter blades and the feasibility of using piezoelectric smart materials with modal filters. In particular, this paper examined the flap and torsional coupling effects and individual blade control with feedback control. In another paper, Nitzsche and Breitbach [14] showed that some blade root forces cancel, due to the symmetry of the hub. They found that higher harmonic control performed poorly at suppressing vibration from the hub.

Nitzsche [98] advanced the concept of modifying the stiffness by changing the beam boundary conditions. The beam structure was changed from a hinged system, which has zero stiffness in the flap and feathering directions: first to a flexible link, with a finite flap and feathering stiffness, and second to a cantilever case, with infinite stiffness. The scaled rotor was modelled after a BO-105 helicopter, and experiments were conducted in the German-Dutch Wind Tunnel. Nitzsche $[99,100]$ found that changing the dynamic stiffness at the root of a flexible, bearingless rotor blade was 
an effective method to reduce vibration emanating from the rotor. These papers included a beam model based on Hodges and Dowell; however, modal analysis was employed using an integrating matrix method [101] to reduce the system to single order ordinary differential equations (ODEs). The ODEs were solved with a closedloop control scheme to effectively reduce the vibration from the unsteady aerodynamic loads.

Anusonti-Inthra and Gandhi [102-105] and Anusonti-Inthra's dissertation [106] examined a sinusoidally varying root stiffness to control blade vibration. They analysed a hingeless rotor using the blade properties of a BO-105 helicopter and Drees's (linear) inflow aerodynamics. By changing the baseline stiffness of the blade root, they were able to reduce the vibration in the blades. However, they also described higher harmonics produced and increased blade root moments. With the introduction of springs and dampers, they showed that the flap-lag interaction is the most influential and they were able to reduce vibrations for the first five harmonics.

Combining finite element analysis and positive feedback controllers, Yang, Jiang, and Chen [107] showed that momentum exchange could be used for rigid body control. Harold's thesis [8] outlined the development of a torsional smart spring system. That paper elucidated the efficacy of controlling a twisting structure using a momentum transfer and simple frictional (impedance) control. He also constructed a linear smart spring, the first generation impedance control for the SHARCS project, and developed a state switching control law based on Cunefare [108]. The state-switching control law was examined further by Liu, Waters, and Brennan [109]. They noted that many semi-active controllers are on-off state-switching, and their paper stated that clear performance gains could be had by basic control strategies. 


\subsection{Objectives and Novelty of Research}

Vibrations emanating from the main rotor cause undesirable accelerations in the cabin and cockpit of most rotorcraft. These vibrations are detrimental to the pilot's health, the mechanisms and structure of the helicopter, and cargo or passengers. This research presents a novel method of controlling helicopter vibration transmissibility from the blades to the fuselage. Exchanging the pitch link for a semi-active impedance controller alters the boundary conditions of the kinematically determined rotor blade, and diminishes the impact of the vibration emitted by the main rotor on the helicopter body.

The primary objective of this thesis is to outline the theory for coupling the rigid body articulation with the three dimensional nonlinear elastic blade model. Included in that goal is the inclusion of the entire hub articulation: the aerodynamic chain and the control chain modelled together. The culmination of this objective results in a computer program, b3dsm.m (blade 3-dimensional structural model), which is an m-file written in MATLAB v. 2007b. The computer program is intended to be a research tool, useable independently or coupled with the aerodynamic methods method in the review.

The program is to have the fully coupled parallel kinematics of the hub coupled with the elastic blade at the blade root. All degrees of freedom are allowed in the articulation, including the collective and cyclic pitches, which are typically pilot controlled. The adaptive pitch link is also included, and can be implemented on any simulated flight, or turned off, as the simulation requires. Hover and forward flight aerodynamics are modelled to provide simplified load cases. (Note that the program is designed for constant velocities; in any accelerating flight the assumption that the inertial frame of reference attached to the hub is no longer valid, neither are the 
aerodynamics.)

The topics in this thesis cover four main groups: rotor blade elastic dynamics, blade aerodynamics, rigid body mechanics, and vibration control. Individually, the topics described in this thesis are not wholly new: as exemplified in the review, proposals have been made for each component in some field. However, many of the concepts are relatively new to the helicopter research field, and no research exists that combines these topics coherently. Within these broad topics are novel nuances that are not normally discussed in rotorcraft forums, such as the coupling between the rotor blade itself or the dual chain articulated hinges.

The rotor flexible dynamics use three-dimensional elements. Commonly, rotor blade dynamics are modelled as beam elements, or combined in an asymptotic method common to "one-dimensional plus two-dimensional" analyses. The choice of a threedimensional finite element model is due to its simplicity and accuracy for full aeroelastic effects. The generalized- $\alpha$ energy momentum method (GEMM) is quite new, and stabilizes the non-linear equations of motion better than previous schemes. In non-linear structural dynamics, the stability of the algorithm is the main interest, because unconditionally stable algorithms in linear dynamics are not necessarily so in non-linear dynamics. For non-linear systems, a sufficient condition for stable solutions is that the energy must be constant, or decay, within a time step. (Spectral stability is required also, as a necessary condition.) The GEMM concurrently allows energy conservation or decay and controllable numerical dissipation of unwanted high frequency modes with second order accuracy.

Simplified blade aerodynamics are very commonly done for helicopter research papers, typically for hover or Drees (linear) inflow models. These analyses usually don't involve three-dimensional structural models. Often the aerodynamics package uses a beam model; however, there are some models that use shell elements. The analysis 
presented in this thesis describes the non-linear Mangler and Squire inflow model, which more accurately shows the inflow at the centre and edges of the rotor disc. As part of the SHARCS project, in this thesis, the aerodynamics package is simply a substitute for a complex aerodynamics analysis, which is done with SMARTROTOR.

Articulation is relatively new in rotorcraft forums, but the Denavit-Hartenberg formulation for rigid body frames of reference allow easy computation of relative frames. Decidedly uncommon in vehiclar dynamics software is the introduction of a closedkinematic chain; only a few of these software packages deal with closed-kinematic chains, and these packages require expertise in manipulating the individual members of the mechanism. Unfortunately, due to their competitive commercial nature, they are also difficult to verify and it is expensive to purchase licences. Other programs that have been proven for structural mechanics require a user to know boundary conditions a priori, in order to calculate forces. In this research, the articulation boundary conditions are dependent on the excitation forces, which are in turn dependent on the boundary conditions. For example, switching between two values of stiffness, in addition to the mass and damping differences, changes based on the forces introduced to the hinge system from the blade. This iterative process is slow and error prone when done in typical structural solvers not appropriately set up to deal with such issues.

The coupling between the rigid mechanics and the elastodynamic blade is also novel. At the blade root, the internal forces of the elastic body rotor blade must balance the external forces arising from the rigid body articulation. The force resultant on the blade root depends on the position and orientation of the blade, which is determined by the hub articulation. The hub articulation is dependent on the force resultant to balance the torques prescribed by the external forces from the blade. Again, an iterative procedure could solve this root boundary condition, but with the 
same drawbacks as mentioned above.

The program described in this thesis is unique in its ability to solve complicated geometric boundary conditions with internal reaction forces. This is necessary for the current research, as the reaction forces developed from the blade-hub coupling are inputs into the rigid body dynamics of the hub. Currently, no other three-dimensional solver exists with an articulated hub model, let alone a closed-kinematic chain.

Finally, root control strategies exist in literature; however, no root control proposal exists that suggests the replacement of the pitch link with an active controller, except those from the SHARCS project. The impedance control device is an innovative way to reduce the vibration transmissibility from the blades to the fuselage. Exchanging the pitch link with the APL provides semi-active control that is lightweight, cheap, easy to implement on existing rotorcraft, and effective. Previous root control concepts involve modification of the blade at the root, not at its attachment to the pilot control, and none of those models matches the complexity of the articulation model that is described in this dissertation. In addition, no model has proposed a discrete, timedependent boundary condition modification.

The research collated within this thesis represents a collection of ideas from many fields applied to a full rotor head. By themselves, each section constitutes an improvement in tools used for understanding rotorcraft as a complex global system. Collectively, this research constitutes a push forward in the understanding of how each aspect of the main rotor interacts with the other systems. 


\section{Chapter 2}

\section{General Continuum Mechanics and Finite Element Development for Solid Structures}

This chapter provides a brief description of continuum mechanics as background for the nonlinear finite element solution. This work cannot cover the entire breadth of continuum mechanics and finite element analysis; however, some basics are necessary for comprehensiveness. There are many good references for further reading on continuum mechanics, such as Bonet and Wood [41], or Haddad [42]. The work on the finite element discretization comes from Bornemann $[46,47]$, while the time discretization schemes are based on the same and from $[48,110]$.

Nonlinearity comes from large physical deformation (geometric), nonlinear constitutive relationships (material), and from nonlinear external forces or displacements (boundary conditions). Geometric nonlinearity is a physical change in the structure such that the deformation is large enough to warrant setting up the equilibrium equations in the deformed position. The other sources of nonlinearity described in this dissertation arise from force boundary conditions, specifically from the motion of the articulation and the friction of the APL, but this chapter only outlines the elastic body geometric nonlinearity. 


\subsection{Strain and Deformation}

A relationship between the deformation of an object and its original description begins with strain, a measure of the distortion of a body. Figure 2.1 shows a continuum material displaced and deformed over a period of time relative to its original position. The diagram shows two ways the object could be referenced: with respect to the current (deformed) frame $\mathbf{x}$, or the initial (undeformed) frame $\mathbf{X}$. (Note that in this chapter only, miniscule characters represent spatial coordinates, whereas majuscule characters denote material coordinates.) Here, it is assumed that the two mapping systems are referred to a common Cartesian coordinate frame. Although not strictly necessary, as long as a mapping function between the two systems is known, having a coincident frame of reference simplifies the relationship between the two frames.

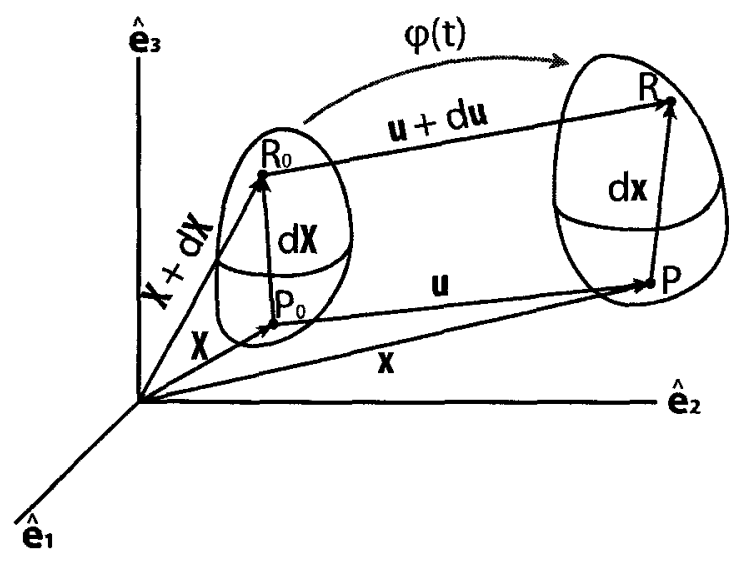

Figure 2.1: Undeformed and deformed configurations of a body described in coincidental frames of reference.

Using a coincedent frame of reference, a point in space can therefore be represented by the current position of the particle as

$$
\mathbf{x}=x_{1} \hat{\mathbf{e}}_{1}+x_{2} \hat{\mathbf{e}}_{2}+x_{3} \hat{\mathbf{e}}_{3}=x_{k} \hat{\mathbf{e}}_{k}
$$


or the initial configuration as

$$
\mathbf{X}=X_{1} \hat{\mathbf{e}}_{1}+X_{2} \hat{\mathbf{e}}_{2}+X_{3} \hat{\mathbf{e}}_{3}=X_{k} \hat{\mathbf{e}}_{k}
$$

in which $\hat{\mathbf{e}}_{k}$ represents the Cartesian basis for the current position frame of reference and the unit basis for the initial description, because the coordinate systems are superimposed. From these definitions, a displacement vector $\mathbf{u}$ or $\mathbf{U}$, depending on the description, can be expressed as either

$$
\mathbf{u}=u_{k} \hat{\mathbf{e}}_{k} \quad \text { or } \quad \mathbf{U}=U_{k} \hat{\mathbf{e}}_{k}
$$

and the relationship between the two positions can be given as

$$
\mathbf{u}=\mathbf{x}-\mathbf{X}
$$

If the deformation of the particles in the continuum is expressed in the current configuration, its coordinates are functions of the original configuration. That can be expressed mathematically as

$$
x_{i}=x_{i}\left(X_{1}, X_{2}, X_{3}, t\right)
$$

or

$$
\mathbf{x}=\mathbf{x}(\mathbf{X}, t)
$$

where $t$ is used to denote the time dependent aspect of deformation.

This expression can also be interpreted as the map of the initial configuration onto the current configuration. Referencing the deformed system is known as the material, 
or Lagrangian, formulation.

Conversely, if the deformation is expressed in the original configuration, its coordinates are functions of the current configuration. That results in the following expression:

$$
X_{i}=X_{i}\left(x_{1}, x_{2}, x_{3}, t\right)
$$

or

$$
\mathbf{X}=\mathbf{X}(\mathbf{x}, t)
$$

which is known as the spatial, or Eulerian, description.

These maps are both one-to-one and continuous, with continuous partial derivatives to whatever degree necessary. The maps are also unique inverses of one another. It is important that these continuum configuration descriptions can be related to each other, because a deformation gradient is necessary to describe the changes a group of particles undergo through the transformation $\phi(t)$, and both descriptors are required.

\section{Deformation Gradient}

The material deformation gradient, $\mathbf{F}$, relates the initial state of an infinitesimal fibre $d \mathbf{X}$, to its current state $d \mathbf{x}$. Mathematically, it is the partial derivative of the Lagrangian tensor by the Eulerian. That is,

$$
\mathbf{F}=\mathbf{x} \nabla_{\mathbf{x}} \equiv \frac{\partial \mathbf{x}}{\partial X_{1}} \hat{\mathbf{e}}_{1}+\frac{\partial \mathbf{x}}{\partial X_{2}} \hat{\mathbf{e}}_{2}+\frac{\partial \mathbf{x}}{\partial X_{3}} \hat{\mathbf{e}}_{3}
$$


Going back to the initial configuration, the distance between neighbouring particles, $P_{0}$ and $R_{0}$, can be expressed as the square of the undeformed coordinate $\mathbf{X}$ :

$$
d \mathbf{X}^{2}=d \mathbf{X} \cdot d \mathbf{X}=\delta_{i j} d X_{\imath} d X_{\jmath}
$$

Looking again at particles $P$ and $R$ (in the deformed configuration) in Figure 2.1, the square of the differential length is

$$
d \mathbf{x}^{2}=d \mathbf{x} \cdot d \mathbf{x}=\delta_{\imath \jmath} d x_{i} d x_{\jmath}
$$

and as $d x_{\imath}$ is a function of the initial coordinates system,

$$
d x_{\imath}=\frac{\partial x_{i}}{\partial X_{\jmath}} d X_{\jmath}
$$

or

$$
d \mathbf{x}=\mathbf{F} d \mathbf{X}
$$

then the squared length becomes

$$
(d \mathbf{x})^{2}=\frac{\partial x_{k}}{\partial X_{\imath}} \frac{\partial x_{k}}{\partial X_{j}} d X_{i} d X_{j}=d \mathbf{X}^{\top} \mathbf{F}^{\top} \mathbf{F} d \mathbf{X}
$$

Strain, in the one-dimensional classical mechanics sense, is the stretch of a body relative to the body's original length. It measures the deformation of an object, by relating the deformed position and the original, undeformed position. The strain on the material between $P$ and $R$ is the difference in the distance between the neighbouring particles after loading. If this value is zero, then no deformation has occurred, only rigid body displacements. 
With the two above coordinate frames in mind, taking the difference results in

$$
\begin{aligned}
d \mathbf{x}^{2}-d \mathbf{X}^{2} & =\left(\frac{\partial x_{k}}{\partial X_{i}} \frac{\partial x_{k}}{\partial X_{j}}-\delta_{i j}\right) d X_{i} d X_{j} \quad \text { or } \\
& =d \mathbf{X}^{\top}\left(\mathbf{F}^{\top} \mathbf{F}-\mathbf{I}\right) d \mathbf{X} \\
& =2 d \mathbf{X}^{\top} \varepsilon_{G L} d \mathbf{X}
\end{aligned}
$$

where $\mathbf{I}$ is the identity tensor and $\varepsilon_{G L}$, also written as $\mathbf{E}$, is the Green-Lagrange finite strain tensor, defined as

$$
\mathbf{E}=\varepsilon_{G L}=\frac{1}{2}\left(\mathbf{F}^{\top} \mathbf{F}-\mathbf{I}\right)
$$

Recalling Equation (2.4) and re-arranging it, gives

$$
\mathbf{x}=\mathbf{X}+\mathbf{u}
$$

which, using Equation (2.10), can be explicitly given as

$$
\mathbf{F}=\frac{\partial \mathbf{x}}{\partial \mathbf{X}}=\mathbf{I}+\frac{\partial \mathbf{u}}{\partial \mathbf{X}}=\mathbf{I}+\nabla_{\mathbf{X}} \mathbf{u}
$$

This results in the strain expressed in terms of the deformation as

$$
\begin{aligned}
\mathbf{E} & =\frac{1}{2}\left(\frac{\partial \mathbf{u}}{\partial \mathbf{X}}+\left(\frac{\partial \mathbf{u}}{\partial \mathbf{X}}\right)^{\top}+\left(\frac{\partial \mathbf{u}}{\partial \mathbf{X}}\right)^{\top} \frac{\partial \mathbf{u}}{\partial \mathbf{X}}\right) \\
& =\frac{1}{2}\left(\nabla_{\mathbf{X}} \mathbf{u}+\left(\nabla_{\mathbf{X}} \mathbf{u}\right)^{\top}+\left(\nabla_{\mathbf{X}} \mathbf{u}\right)^{\top}\left(\nabla_{\mathbf{X}} \mathbf{u}\right)\right)
\end{aligned}
$$

Now that a strain measure has been determined, the appropriate corresponding stress needs to be analysed. Specific stress-strain pairs form energy conjugates, which describe the same internal energy density carried by the material. In the case of the 
Green-Lagrange strain, the energy complimenting stress is the Second Piola-Kirchhoff stress tensor.

\subsection{Stress}

The Second Piola-Kirchhoff stress tensor can be derived from Cauchy's Stress Principle, which postulates that the ratio of a finite force applied over a finite area approaches a definite limit. Referring to Figure 2.2, the body depicted undergoes some body force $\mathbf{b}$, and an applied surface force $\mathbf{f}$. As the surface area $d s$, vanishes at the point of interest, the differential force $d \mathbf{f}$, becomes the traction vector $\mathbf{t}^{\hat{\mathbf{n}}}$, which is the Cauchy limit. Explicitly, this is

$$
\mathbf{t}^{\hat{\mathbf{n}}}=\lim _{d s \rightarrow 0} \frac{d \mathbf{f}}{d s}
$$

Traction is distinguished from stress because it is dependent on the infinitesimal surface element, which is also why there is a superscript to denote its basis.

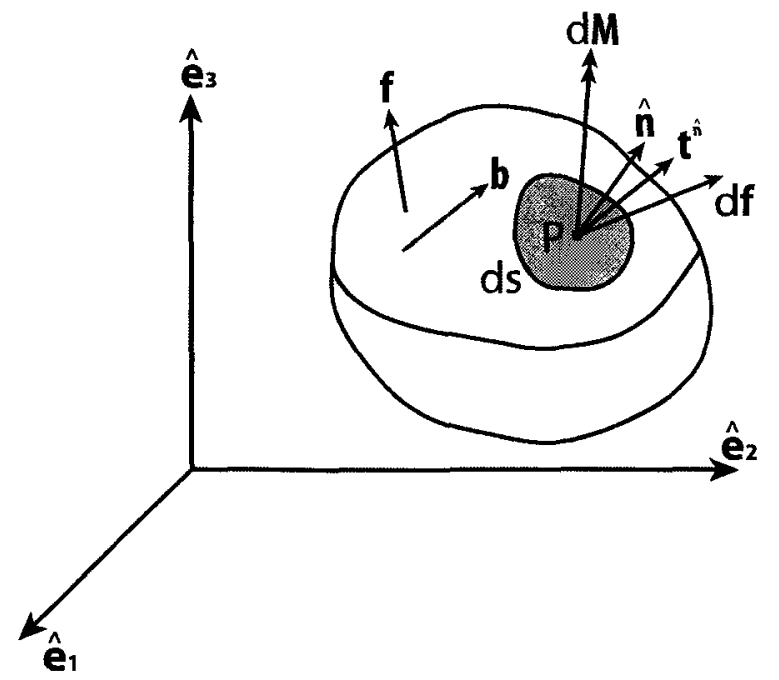

Figure 2.2: Stress elements on globular body. 
Cauchy's stress principle associates the stress tensor with each unit normal, which represents the orientation of the surface elements with $\mathrm{P}$ as the interior point. All possible pairs of tensors $\mathbf{t}^{\hat{\mathbf{n}}}$ and $\hat{\mathbf{n}}$ define the state of stress at Point $\mathrm{P}$, and can be described by using three orthonormal bases. Transformations associate the traction to each plane with respect to the others, and is written in Cartesian coordinates based on the material description as

$$
\begin{aligned}
& \mathbf{t}^{\hat{\mathbf{e}}_{1}}=t_{1}^{\hat{\mathbf{e}}_{1}} \hat{\mathbf{e}}_{1}+t_{2}^{\hat{\mathbf{e}}_{1}} \hat{\mathbf{e}}_{2}+t_{3}^{\hat{\mathbf{e}}_{1}} \hat{\mathbf{e}}_{3} \\
& \mathbf{t}^{\hat{\mathbf{e}}_{2}}=t_{1}^{\hat{\mathbf{e}}_{2}} \hat{\mathbf{e}}_{1}+t_{2}^{\hat{\mathbf{e}}_{2}} \hat{\mathbf{e}}_{2}+t_{3}^{\hat{\mathbf{e}}_{2}} \hat{\mathbf{e}}_{3} \\
& \mathbf{t}^{\hat{\mathbf{e}}_{3}}=t_{1}^{\hat{\mathbf{e}}_{3}} \hat{\mathbf{e}}_{1}+t_{2}^{\hat{\mathbf{e}}_{3}} \hat{\mathbf{e}}_{2}+t_{3}^{\hat{\mathbf{e}}_{3}} \hat{\mathbf{e}}_{3} .
\end{aligned}
$$

These nine elements are the components to the traditional stress tensor, which is also called the Cauchy stress tensor. The Cauchy stress tensor can be compactly written from Cauchy's theorem,

$$
\mathbf{t}^{\hat{\mathbf{n}}}=\boldsymbol{\sigma}_{C}^{\top} \cdot \hat{\mathbf{n}}
$$

where the Cauchy stress $\sigma_{C}$, can be expanded in the familiar form:

$$
\sigma_{C_{i j}}=\left[\begin{array}{ccc}
\sigma_{11} & \sigma_{12} & \sigma_{13} \\
\sigma_{21} & \sigma_{22} & \sigma_{23} \\
\sigma_{31} & \sigma_{32} & \sigma_{33}
\end{array}\right]
$$

The Cauchy stresses describe the state of stress at the present (deformed) configuration, which is not known beforehand; however, the deformation gradient and GreenLagrange strains reference the undeformed system. Therefore, Cauchy stresses must be mapped to the initial configuration if a coherent energy state is to be defined. The First Piola-Kirchhoff stress tensor maps one index of the Cauchy stresses to the 
initial configuration by

$$
\sigma_{P K 1}=\operatorname{det}(\mathbf{F}) \sigma_{C} \mathbf{F}^{-\top}
$$

but this stress measure relates the current force system to the initial state area. Therefore, this stress tensor is not symmetric, and still not suitable to use with the Green-Lagrange strain.

The Second Piola-Kirchhoff stress tensor relates both the force and the area to the initial configuration. Thus, it is a symmetric conjugate to the Green-Lagrange strain and it is related to the Cauchy stress by the transformation

$$
\mathbf{S}=\boldsymbol{\sigma}_{P K 2}=\operatorname{det}(\mathbf{F}) \mathbf{F}^{-1} \sigma_{C} \mathbf{F}^{-\mathbf{T}}
$$

where $\mathbf{S}$ often replaces the $\sigma_{P K 2}$ notation for the Second Piola-Kirchhoff stress tensor. A note on the Second Piola-Kirchhoff stress: the map preserves the force and area relationship from the current configuration to the initial, but the diagonal components are not simply the normal stresses, nor are the off-diagonal terms the total shear stresses. From Figure 2.3, it is clear that the diagonal components of the stress tensor are not normal to the undeformed surface element, nor are the shear stresses parallel to the edges of the original element.

\subsection{Governing Laws of Mechanics}

Now that the concept of strains and stresses have been elucidated in terms of continuum mechanics, the overall relationships governing the equations of motion can be determined. There are three sets of equations that describe the equations of motion, the kinematics equations, the balance equations, and the constitutive equations. The 


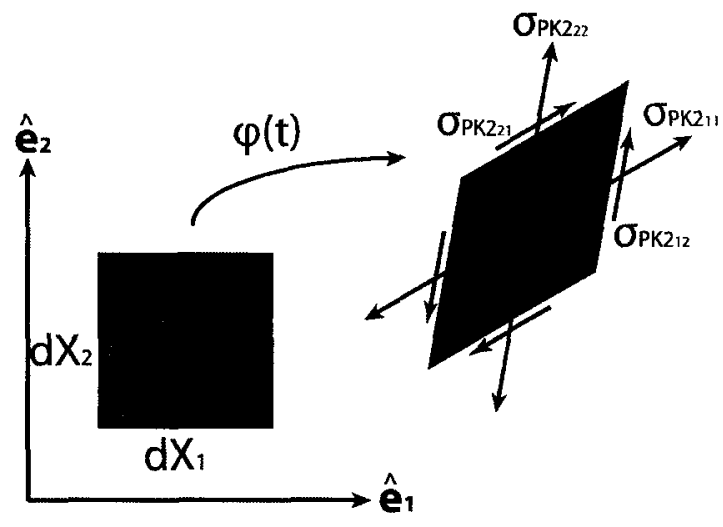

Figure 2.3: 2nd Piola-Kirchhoff stress tensor directions. Adapted from [46].

kinematic equations arise from the relationships of strain and displacement. The balance and constitutive equations come from the combination of the Laws of Conservation of Mass, Momentum, and Energy.

\section{Kinematic Equations}

As mentioned, the kinematic equations relate the strains to the displacements. The Green-Lagrange strain is a function of the deformation gradient, which in turn is a function of the displacements. Therefore, the Green-Lagrange strain is inherently a nonlinear relationship between the strain and the displacements. As a reminder the kinematic equations in the material description are

$$
\mathbf{E}=\frac{1}{2}\left(\mathbf{F}^{\top} \mathbf{F}-\mathbf{I}\right)=\frac{1}{2}\left(\nabla_{0} \mathbf{u}+\left(\nabla_{0} \mathbf{u}\right)^{\top}+\left(\nabla_{0} \mathbf{u}\right)^{\top}\left(\nabla_{0} \mathbf{u}\right)\right)
$$

For physically restrained problems, the appropriate boundary conditions apply typically along the surface of the body. 


\section{Balance Equations}

The balance equations are the first set of equations derived from the Conservation Laws. The Conservation of Mass requires that the mass of a contained portion of the continuum must remain constant. It is written, in the material description as the integral of density over the control volume,

$$
\int_{V} \rho_{0}(\mathbf{X}, 0) d V=\int_{v} \rho(\mathbf{x}, \mathrm{t}) \mathrm{dv} .
$$

As with the coordinates $\mathbf{X}$ and $\mathbf{x}$, majiscule variables are referred to the initial coordinate frame, and miniscule variables denote the deformed frame of reference. Hence, the initial volume in Equation (2.24) is $\mathrm{V}$ and the deformed volume is $\mathrm{v}$.

Implicitly, length and area maps have already been encountered in the section on strain and stress; however, in Equation (2.24) a volumetric map must be derived. Derivations can be found in various texts, such as $[41,42]$, but a table adapted from Bornemann [47] summarizes these maps as shown. Table 2.1 reads to show that

Table 2.1: Dimensional maps between original and deformed configurations [47].

\begin{tabular}{cccc} 
& Deformed ref. & Mapping operator & Initial ref. \\
\hline Line element & $\mathrm{d} \mathbf{x}$ & $\mathbf{F}$ & $\mathrm{d} \mathbf{X}$ \\
Area element & $\mathrm{d} \mathbf{a}$ & $\operatorname{det}(\mathbf{F}) \mathbf{F}^{-\mathbf{T}}$ & $\mathrm{d} \mathbf{A}$ \\
Volume element & $\mathrm{d} \mathbf{v}$ & $\operatorname{det}(\mathbf{F})$ & $\mathrm{dV}$ \\
\hline
\end{tabular}

$$
\mathrm{dv}=\operatorname{det}(\mathbf{F}) \mathrm{dV}
$$


or if the Jacobian, $J$, is defined as the determinant of the deformation gradient,

$$
\mathrm{dv}=\mathrm{JdV} .
$$

(It is common, but unfortunate, that the deformation gradient determinant is called the Jacobian, because the Jacobian matrix is used later to evaluate the derivatives of the virtual displacement with respect to the material coordinates. The former Jacobian is a scalar and denoted as $J$, but the latter is a matrix and distinguished in expressions in bold as $\mathbf{J}$.)

Therefore, Eq. (2.24) becomes

$$
\int_{V} \rho_{0}(\mathbf{X}, 0) \mathrm{dV}=\int_{\mathbf{v}} \rho(\mathbf{X}, \mathrm{t}) \mathrm{JdV}
$$

and, because this holds for any volume,

$$
\rho_{0}=\rho J
$$

or

$$
\frac{\mathrm{d}}{\mathrm{dt}}(\rho J)=0
$$

if the initial density is also constant in time. Equation (2.29) is the Lagrangian form of the continuity equation; it is used in combination with the derivation of the Conservation of Momentum.

Referring to Figure 2.4, the balance equations can be derived using the force equilibrium of the continuum as the sum of differential forces and the mass inertia of 


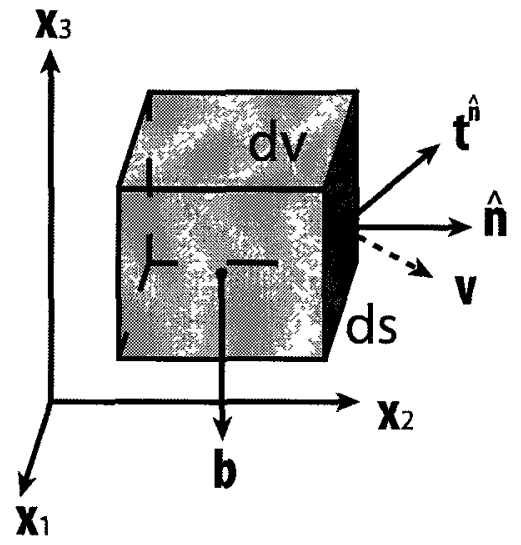

Figure 2.4: Undeformed and deformed configurations of a body.

the infinitesimal element, which is the Conservation of (Linear) Momentum:

$$
\int_{s} \mathbf{t}^{\hat{\mathbf{n}}} \mathrm{ds}+\int_{\mathrm{v}} \rho \mathbf{b d v}=\frac{\mathrm{d}}{\mathrm{dt}} \int_{\mathrm{v}} \rho \mathbf{v d v}
$$

Substituting into the first term of Eq. (2.30) the appropriate stress and basis for the traction tensor, Gauss's divergence theorem for surface integrals states

$$
\int_{s} \boldsymbol{\sigma}^{\top} \cdot \hat{\mathbf{n}} \mathrm{ds}=\int_{\mathbf{v}} \nabla_{\mathbf{x}} \cdot \boldsymbol{\sigma}^{\top} \mathrm{dv}
$$

so that Equation (2.30) is written as

$$
\int_{v}\left(\nabla_{\mathbf{x}} \cdot \boldsymbol{\sigma}^{\mathbf{T}}+\rho \mathbf{b}\right) \mathrm{dv}=\frac{\mathrm{d}}{\mathrm{dt}} \int_{\mathrm{v}} \rho \mathbf{v d v}
$$

The velocity term requires some added attention, since

$$
\frac{\mathrm{d}}{\mathrm{dt}} \int_{v} \rho \mathbf{v d v}=\frac{\mathrm{d}}{\mathrm{dt}} \int_{\mathrm{V}} \rho_{0} \mathrm{JvdV}=\int_{\mathrm{V}}\left[\mathbf{v} \frac{\mathrm{d}}{\mathrm{dt}}\left(\rho_{0} \mathrm{~J}\right)+\rho_{0} \mathrm{~J} \frac{\mathrm{d} \mathbf{v}}{\mathrm{dt}}\right] \mathrm{dV}
$$


which, with the result from the mass continuity equation, means

$$
\frac{\mathrm{d}}{\mathrm{dt}} \int_{v} \rho \mathrm{vdv}=\int_{\mathrm{v}} \rho \frac{\mathrm{d} \mathbf{v}}{\mathrm{dt}} \mathrm{dv}
$$

Collecting the terms gives the integral form of the balance equations:

$$
\int_{V}\left(\nabla_{\mathbf{x}} \cdot \boldsymbol{\sigma}^{\top}+\rho \mathbf{b}-\rho \dot{\mathbf{v}}\right) \mathrm{d} \mathbf{v}=0
$$

where the dot-symbol shows the explicit time derivative of the variable. Since the volume is arbitrary, again the integrand must vanish, so Eq. (2.34) becomes

$$
\nabla_{\mathbf{x}} \cdot \boldsymbol{\sigma}+\rho \mathbf{b}-\rho \dot{\mathbf{v}}=0
$$

which is the pointwise form of the balance equation of motion.

\section{Constitutive Equations}

The constitutive equation can be derived in a similar manner as above, which leads to the full form energy equation for the material:

$$
\frac{\mathrm{du}}{\mathrm{dt}}=\frac{1}{\rho} \boldsymbol{\sigma}: \dot{\varepsilon}-\frac{1}{\rho} \nabla \cdot \mathbf{c}+\mathbf{b} \cdot \mathbf{v}+z,
$$

in which $u$ is the specific internal energy, $\dot{\varepsilon}$ is the strain rate, $\mathbf{c}$ is the heat flux per unit area, and $z$ is the radiant heat constant per mass per unit time. If thermal effects are ignored, the internal energy is purely mechanical and the energy equation becomes

$$
\mathrm{du}=\frac{1}{\rho} \boldsymbol{\sigma}: \mathrm{d} \boldsymbol{\varepsilon} .
$$


To evaluate this expression, it is necessary to have a measure of the strain energy density per current unit volume, such as

$$
u^{*}=\rho u
$$

but for small strain theory, $\rho$ is constant and

$$
\frac{\partial u^{*}}{\partial \varepsilon}=\rho \frac{\partial u}{\partial \varepsilon}
$$

The stress must vanish with the strains, so the simplest strain energy function is quadratic. Choosing

$$
u^{*}=\frac{1}{2} \varepsilon: \mathbf{C}: \varepsilon
$$

where $\mathbf{C}$, which is a $4^{\text {th }}$-order tensor called the constitutive (or stiffness) tensor, represents the elastic constraints. Substituting it into Equation (2.37) with Eq. (2.39) produces the constitutive equation:

$$
\boldsymbol{\sigma}=\mathbf{C}: \varepsilon
$$

This may be clearer in matrix notation, with repeated indices indicating summation,

$$
\sigma_{i j}=C_{i j k l} \varepsilon_{k l}
$$

A general $4^{\text {th }}$ order tensor with three spatial dimensions has 81 component terms, but because of the symmetry of the stress and strain tensors, the number of those components can be reduced to 36 . The constitutive tensor simplifies further upon the modification of the stress and strain notation to 


$$
\left.\begin{array}{ccc}
\sigma_{11}=\sigma_{1} & \sigma_{22}=\sigma_{2} & \sigma_{33}=\sigma_{3} \\
\sigma_{12}=\sigma_{21}=\sigma_{4} & \sigma_{13}=\sigma_{31}=\sigma_{5} & \sigma_{23}=\sigma_{32}=\sigma_{6}
\end{array}\right\}
$$

and

$$
\left.\begin{array}{ccc}
\varepsilon_{11}=\varepsilon_{1} & \varepsilon_{22}=\varepsilon_{2} & \varepsilon_{33}=\varepsilon_{3} \\
\varepsilon_{12}=2 \varepsilon_{21}=\varepsilon_{4} & 2 \varepsilon_{13}=2 \varepsilon_{31}=\varepsilon_{5} & 2 \varepsilon_{23}=2 \varepsilon_{32}=\varepsilon_{6}
\end{array}\right\},
$$

wherein the subscripts follow the rule, $2 \sigma_{i j}=\sigma_{i+j+1}$ for shear stresses and strains. (Note the factor 2 that modifies the shear strain terms, which comes about from the geometry of the shear strain; specifically, due to work conjugacy. Otherwise, the subscript ordering scheme on the stress vector is arbitrary, in that one could chose any distinct subscripts to distinguish stresses.) Using Voigt notation, one can write the stress and strain tensors as matrices, which simplifies the programming required on a computer. With this notation, the constitutive equation can be recast so that the stiffness tensor $\mathbf{C}$ can be rewritten as a $6 \times 6$ constitutive matrix, which is also symmetric, and is used extensively in the future derivation. This symmetry reduces again the number of components, now down to 21. For a fully anisotropic material, that is the maximum number of independent elastic components. Within the remainder of this dissertation, only linear isotropic materials are examined for analysis; therefore, there are only 2 independent elastic constants called Lamé parameters.

For an isotropic Saint Venant-Kirchhoff material the stiffness tensor can be written as

$$
\mathbf{C}=2 \mu \mathbf{I}+\lambda \boldsymbol{\delta} \otimes \boldsymbol{\delta},
$$


where the Lamé parameters are

$$
\begin{aligned}
& \lambda=\frac{\nu E}{(1+\nu)(1-2 \nu)} \\
& \mu=G=\frac{E}{2(1+\nu)},
\end{aligned}
$$

the $4^{\text {th }}$-order identity tensor is

$$
\mathbf{I}=I_{i j k l} \hat{\mathbf{e}}_{i} \otimes \hat{\mathbf{e}}_{j} \otimes \hat{\mathbf{e}}_{k} \otimes \hat{\mathbf{e}}_{l}=\frac{1}{2}\left(\delta_{i j} \delta_{k l}+\delta_{i l} \delta_{j k}\right) \hat{\mathbf{e}}_{i} \otimes \hat{\mathbf{e}}_{j} \otimes \hat{\mathbf{e}}_{k} \otimes \hat{\mathbf{e}}_{l}
$$

and the delta-Dirac is

$$
\boldsymbol{\delta}=\delta_{i j} \hat{\mathbf{e}}_{i} \otimes \hat{\mathbf{e}}_{j}
$$

Written out, the stiffness matrix is

$$
\mathbf{C}=\frac{E}{(1+\nu)(1-2 \nu)}\left[\begin{array}{cccccc}
1-\nu & \nu & \nu & 0 & 0 & 0 \\
\nu & 1-\nu & \nu & 0 & 0 & 0 \\
\nu & \nu & 1-\nu & 0 & 0 & 0 \\
0 & 0 & 0 & \frac{1-2 \nu}{2} & 0 & 0 \\
0 & 0 & 0 & 0 & \frac{1-2 \nu}{2} & 0 \\
0 & 0 & 0 & 0 & 0 & \frac{1-2 \nu}{2}
\end{array}\right]
$$

in which $E$ is Young's modulus and $\nu$ is Poisson's ratio.

The kinematic, balance, and constitutive equations constitute a complete set of relations that describe the motion of the continuum body. These equations, with appropriate boundary conditions, make up an initial-boundary value problem. A finite element solution of the equations can be determined after combining them together in the Principle of Virtual Work and reforming them for a displacementbased approximation. 


\subsubsection{Principle of Virtual Work in the Material Frame}

Displacements, surface tractions, and body forces are the data normally prescribed in a mechanical engineering problem, as is the case for the helicopter blade motion. The displacements are the variables with which the finite element analysis is concerned, and the strains and stresses are secondary variables derived from the displacements. Therefore, the formulation for finite element analysis presented now is based on the displacement variables. The other terms, stress and strain, are written as functions of the displacements.

The general solution starts with the set of partial differential equations derived in the previous sections. The method of weighted residuals is employed next, whereby the balance equations are weighted with test functions, and integrated so that they need only be satisfied in an integral sense. The reason for this is that the test functions can be thought of as Lagrange multipliers to liken them to physical parameters. Furthermore, if they are thought of as a displacement field, then the balance equations becomes an energy expression. Since the test functions are arbitrary except on the boundaries, they can be used as virtual displacements. The equations are 'weakened' by reducing the requirement of the test functions and trial spaces, hence these equations are often called the weak form of the virtual work equations.

Finite dimensional subspaces discretize the weak form of the equations of motion, after which a functional basis for the elemental subspaces can be applied to yield a finite element formulation. The entire process is shown pictorially, including the resulting deformation, in Figure 2.5.

Before the details of this process can be discussed, the starting equations in the material form have to presented. So, including the traction boundary loads, the 
weighted residual for the balance equations becomes

$$
\int_{v}\left(\nabla_{\mathbf{x}} \cdot \boldsymbol{\sigma}+\rho \mathbf{b}-\rho \dot{\mathbf{v}}\right) \mathbf{w d v}+\int_{\mathbf{a}}\left(\mathbf{t}^{\hat{\mathbf{n}}}-\boldsymbol{\sigma} \cdot \hat{\mathbf{n}}\right) \mathbf{w d a}=0
$$

where $\mathbf{w}$ are the weighting functions. Instead, as mentioned, if the weighting functions are considered to be virtual displacements, they can be replaced by $\delta \mathbf{u}$. After applying Gauss' divergence theorem, the expression in the deformed coordinate system is

$$
\delta W=\int_{v} \boldsymbol{\sigma}: \delta \mathbf{e d v}+\int_{\mathbf{v}} \rho \dot{\mathbf{v}} \cdot \delta \mathbf{u d v}-\int_{\mathbf{v}} \hat{\mathbf{b}} \cdot \delta \mathbf{u d v}-\int_{\mathbf{a}} \mathbf{t}^{\hat{\mathbf{n}}} \cdot \delta \mathbf{u d a}=0
$$

Previously, the spatial description was used to derive the balance equations, but here the principle of virtual work is presented in the material form. The virtual work is independent of the configuration with which it is described, and can therefore be expressed with either configuration. Thus, with some manipulation, which can be found in most finite element or continuum mechanics texts, the principle of virtual work in tensor notation is

$$
\delta W=\int_{V} \mathbf{S}: \delta \mathbf{E d V}+\int_{\mathrm{V}} \rho \dot{\mathbf{v}} \cdot \delta \mathbf{u d V}-\int_{\mathrm{V}} \hat{\mathbf{b}}_{0} \cdot \delta \mathbf{u d V}-\int_{\mathrm{A}} \mathbf{t}_{0}^{\hat{\mathbf{n}}} \cdot \delta \mathbf{u d A}=0
$$

To obtain a viable numerical solution to the above equation, it must be discretized in time and space. The separation of variables technique substitutes the displacement field, dependent on time and space, with trial functions such that time dependent and spatially dependent functions can be used. Spatial differential equations are discretized according to finite element methods, while the time-dependent equations are discretized according to finite difference formulae. 


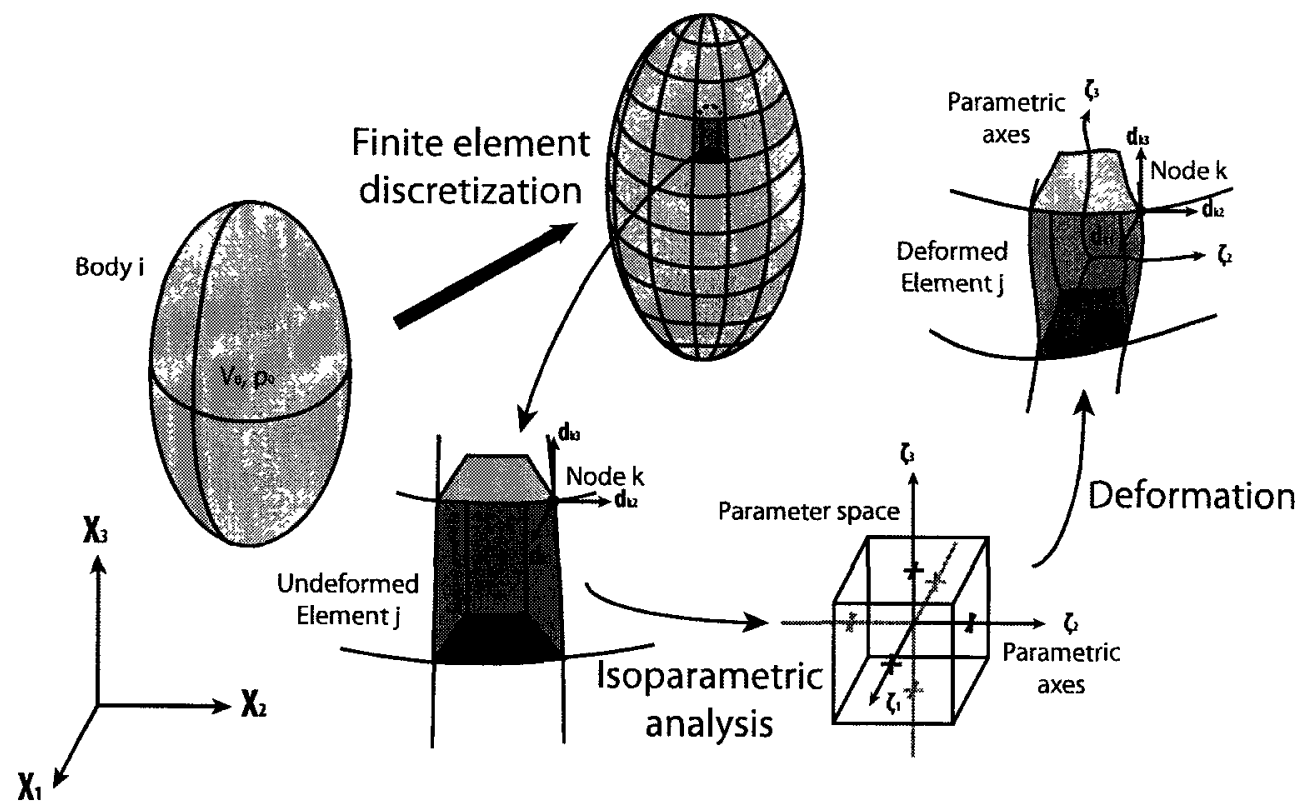

Figure 2.5: Graphical representation of finite discretization process and deformation analysis.

\subsection{Finite Element Discretization}

The finite element mesh must first be applied to the continuum body, which fits the geometry as closely as possible. The undeformed volume splits into nele element volumes $V^{(e)}$, as shown in Figure 2.5, and on each element domain spatial-temporal trial functions $\mathbf{u}^{(e)}$ act. The trial functions are the product of discrete spatial functions, that fit the geometric boundary conditions, and temporal nodal displacement functions as

$$
\mathbf{u}^{(e)}(\zeta, t)=\mathbf{N}^{(e)}(\zeta) \cdot \mathbf{d}^{(e)}(t)
$$

where $\mathbf{N}^{(e)}$ are the spatial shape functions, $\mathbf{d}^{(e)}$ are the discrete time dependent displacements, and the trial functions are applicable within the meshed body. Because 
the space and time functions are separable, spatial derivatives only affect $\mathbf{N}^{(e)}(\mathbf{e})$ and time derivatives only affect $\mathbf{d}^{(e)}(t)$.

As discussed, the virtual displacements are time independent herein, chosen similarly to the trial functions, such that

$$
\delta \mathbf{u}^{(e)}=\mathbf{N}^{(e)}(\zeta) \delta \mathbf{d}^{(e)}
$$

and $\delta \mathbf{u}=\mathbf{0}$ on the Dirichlet boundary. The virtual displacements are functions of tri-quadratic Lagrange polynomials, dependent on the node location within the isoparametric element. They are of the form $-\frac{1}{2}\left(\zeta_{k}-\zeta_{k}^{2}\right)$ for the negative nodes in the isoparametric frame, $\left(1-\zeta_{k}^{2}\right)$ for the centre nodes, and $\frac{1}{2}\left(\zeta_{k}+\zeta_{k}^{2}\right)$ for positive side nodes, with $k=1,2,3$. The 27 combinations and the 81 derivatives necessary to describe the 27-noded element in the isoparametric frame with the standard finite element numbering system can be found in texts, such as Hughes [111]. To visualize the isoparametric element, the negative, middle, and positive nodes are shown in Figure 2.6. For example, using the Figure as a guide, Nodes 1, 13, and 26 have the shape functions,

$$
\begin{aligned}
& N_{1_{(-1,-1,-1)}}=-\frac{1}{8}\left(\zeta_{1}-\zeta_{1}^{2}\right)\left(\zeta_{2}-\zeta_{2}^{2}\right)\left(\zeta_{3}-\zeta_{3}^{2}\right) \\
& N_{13_{(0,-1,1)}}=-\frac{1}{4}\left(1-\zeta_{1}^{2}\right)\left(\zeta_{2}-\zeta_{2}^{2}\right)\left(\zeta_{3}+\zeta_{3}^{2}\right) \\
& N_{26_{(1,0,0)}}=\frac{1}{2}\left(\zeta_{1}+\zeta_{1}^{2}\right)\left(1-\zeta_{2}^{2}\right)\left(1-\zeta_{3}^{2}\right) .
\end{aligned}
$$

Before continuing, the deformation gradient tensor $\mathbf{F}$ should be rewritten in vector form to make use of matrix multiplications, which will make programming simpler. 


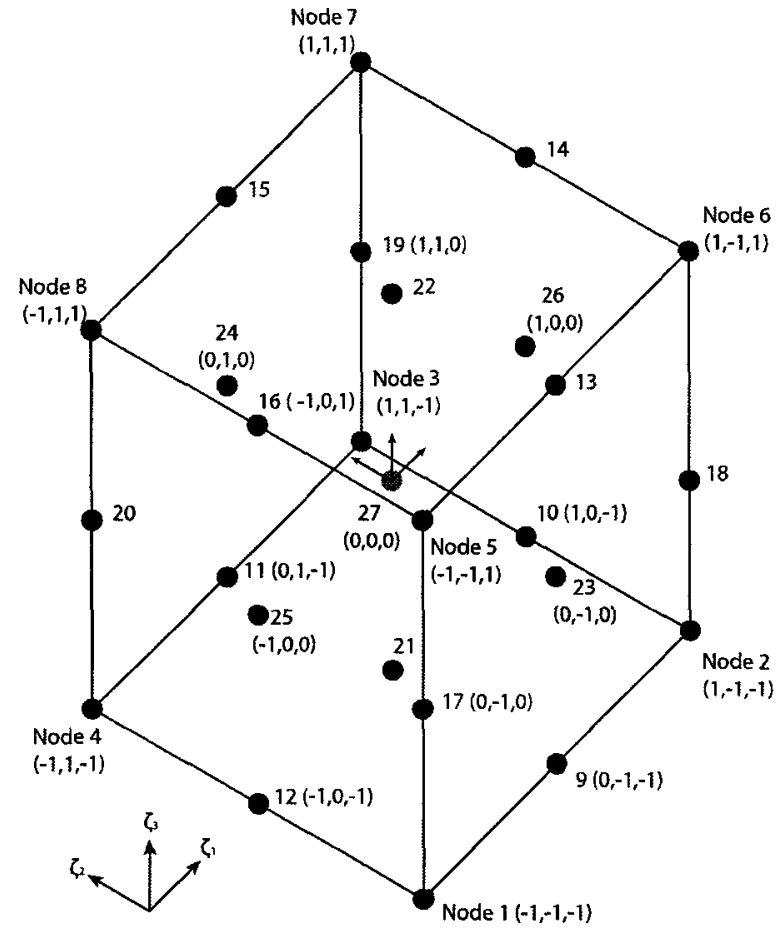

Figure 2.6: The isoparametric numbering system with some of the shape functions.

If the deformation gradient is written as

$$
\mathbf{F}=\left[\begin{array}{lllllllll}
F_{11} & F_{22} & F_{33} & F_{12} & F_{23} & F_{31} & F_{13} & F_{21} & F_{32}
\end{array}\right]^{\top},
$$

and the node shapes are written explicitly as

$$
\mathbf{N}=\left[\begin{array}{cccccccccc}
N_{1} & 0 & 0 & N_{2} & 0 & 0 & \cdots & N_{n} & 0 & 0 \\
0 & N_{1} & 0 & 0 & N_{2} & 0 & \cdots & 0 & N_{n} & 0 \\
0 & 0 & N_{1} & 0 & 0 & N_{2} & \cdots & 0 & 0 & N_{n}
\end{array}\right]
$$

where the number of nodes is $n$, then the deformation gradient is the product of a differential operator and the shape functions plus a vector of ones (normal stress/strain 
directions) and zeroes (transverse stress/strain directions) $\mathbf{1}$, as

$$
\begin{aligned}
\mathbf{F} & =\mathbf{1}+\frac{\partial \mathbf{u}}{\partial \mathbf{X}} \\
& =\mathbf{1}+\mathbf{L N d}
\end{aligned}
$$

where the differential operator looks like:

$$
\mathbf{L}=\left[\begin{array}{ccccccccc}
\partial_{X_{1}} & 0 & 0 & \partial_{X_{2}} & 0 & 0 & \partial_{X_{3}} & 0 & 0 \\
0 & \partial_{X_{2}} & 0 & 0 & \partial_{X_{3}} & 0 & 0 & \partial_{X_{1}} & 0 \\
0 & 0 & \partial_{X_{3}} & 0 & 0 & \partial_{X_{1}} & 0 & 0 & \partial_{X_{2}}
\end{array}\right]^{\top}
$$

Because of the linear dependence of $\mathbf{F}$ on $\mathbf{u}$, it is useful to define $\mathbf{B}_{l \text { in }}$ as

$$
\mathbf{B}_{\text {lin }}=\mathbf{L N}=\frac{\partial \mathbf{F}}{\partial \mathbf{d}}
$$

which comes up often in the derivation of the stress-strain virtual work term.

Since the element shape functions are defined in the parameter domain, but the differentiation occurs with respect to $\mathbf{X}$, a Jacobian relating the two domains is necessary. The Jacobian is

$$
\mathbf{J}=\left[\begin{array}{lll}
\frac{\partial \zeta_{1}}{\partial X_{1}} & \frac{\partial \zeta_{1}}{\partial X_{2}} & \frac{\partial \zeta_{1}}{\partial X_{3}} \\
\frac{\partial \zeta_{2}}{\partial X_{1}} & \frac{\partial \zeta_{2}}{\partial X_{2}} & \frac{\partial \zeta_{2}}{\partial X_{3}} \\
\frac{\partial \zeta_{3}}{\partial X_{1}} & \frac{\partial \zeta_{3}}{\partial X_{2}} & \frac{\partial \zeta_{3}}{\partial X_{3}}
\end{array}\right]
$$

and the transformation is

$$
\begin{aligned}
\frac{\partial \mathbf{e}}{\partial \mathbf{X}} & =\mathbf{J}, & \text { but } \\
\partial \boldsymbol{\zeta} & =\mathbf{J} \partial \mathbf{X} . &
\end{aligned}
$$


Finally, the discrete form of the internal work term $\mathbf{E}: \mathbf{S}$, must be elucidated and rewritten in terms of the virtual displacements. Remembering that in tensor form,

$$
\mathbf{E}=\frac{1}{2}\left(\mathbf{F}^{\top} \mathbf{F}-\mathbf{I}\right)
$$

the virtual strain is

$$
\delta \mathbf{E}=\frac{1}{2}\left(\delta \mathbf{F}^{\top} \mathbf{F}+\mathbf{F}^{\top} \delta \mathbf{F}\right)
$$

which, in Voigt-matrix form, is

$$
\delta \mathbf{E}=\mathbf{F}^{\top} \mathbf{B}_{l i n} \delta \mathbf{d}
$$

because of the ordering scheme of the elemental deformation gradient in matrix form. Therefore, the scalar double dot product yields

$$
\delta \mathbf{E}^{\top} \mathbf{S}=\delta \mathbf{d}^{\top} \mathbf{B}_{l i n}^{\top} \mathbf{F S}
$$

and the internal virtual work can be written in matrix form as

$$
-\delta W=\delta \mathbf{d}^{\top} \int_{V^{(e)}} \mathbf{B}_{l i n}^{\top} \mathbf{F}^{(e)} \mathbf{S} d V
$$

The time derivative only affects the nodal displacements, not the shape functions. Therefore,

$$
\left.\begin{array}{rl}
\dot{\mathbf{u}}^{(e)} & =\mathbf{N}^{(e)}(\boldsymbol{\zeta}) \cdot \dot{\mathbf{d}}^{(e)}(t) \\
\ddot{\mathbf{u}}^{(e)} & =\mathbf{N}^{(e)}(\zeta) \cdot \ddot{\mathbf{d}}^{(e)}(t)
\end{array}\right\},
$$

where the discrete nodal velocities are $\dot{\mathbf{d}}^{(e)}$ and the accelerations are $\ddot{\mathbf{d}}^{(e)}$. 
Combining the results for the variation of the node displacements and the strain energy, the Principle of Virtual Work, Equation (2.50), appears as

$$
\begin{aligned}
\delta W=\sum_{e=1}^{n e l e}\left[\delta \mathbf { d } ^ { \top } \left\{\int_{V^{(e)}} \mathbf{N}^{\top} \rho_{0} \mathbf{N} d V \ddot{\mathbf{d}}\right.\right. & +\int_{V^{(e)}} \mathbf{B}_{l i n}^{\top} \mathbf{F}^{(e)} \mathbf{S} d V-\int_{V^{(e)}} \mathbf{N}^{\top} \hat{\mathbf{b}}_{0} d V \\
& \left.\left.-\int_{A^{(e)}} \mathbf{N}^{\top} \hat{\mathbf{t}}_{0} d A\right\}\right]^{(e)}=0,
\end{aligned}
$$

and the global solution to the finite element problem is assembled as

$$
\begin{aligned}
A_{e=1}^{n e l e}\left[\delta \mathbf{d}^{(e)^{\top}}\left\{\mathbf{m}^{(e)} \ddot{\mathbf{d}}^{(e)}+\mathbf{f}_{i n t}^{(e)}\left(\mathbf{d}^{(e)}\right)-\mathbf{f}_{e x t}^{(e)}\right\}\right] & =0 \\
\delta \mathbf{d}^{\top}\left\{\mathbf{M} \ddot{\mathbf{d}}+\mathbf{f}_{i n t}(\mathbf{d})-\mathbf{f}_{e x t}\right\} & =0
\end{aligned}
$$

where $\mathbf{m}^{(e)}$ is the elemental mass, $\mathbf{f}_{\text {int }}^{(e)}$ is the internal forces vector for the element, $\mathbf{f}_{e x t}^{(e)}$ is the elemental external forces vector, and $\mathbf{M}$ is the global mass matrix. Since the virtual displacements are arbitrary, except on the body boundary, the bracketed terms must vanish, so the finite element based equation of motion is

$$
\mathbf{M} \ddot{\mathrm{d}}+\mathrm{f}_{i n t}(\mathbf{d})-\mathbf{f}_{e x t}=0
$$

and the appropriate boundary conditions apply to the body surface, completing the equation of motion.

\subsubsection{Generalized- $\alpha$ Method for Time Integration}

Now that the spatial elements have been assembled and equated, solving Equation (2.67) requires its time integration. The time-varying solution uses an integration 
scheme based on the finite difference formulae from the Newmark family of parameterization [53], so that the nodal displacements, velocities, and accelerations, are

$$
\begin{aligned}
& \frac{\mathbf{d}_{n+1}-\mathbf{d}_{n}}{\Delta t}=\mathbf{v}_{n}+\frac{\Delta t}{2}\left(2 \beta \mathbf{a}_{n+1}+(1-2 \beta) \mathbf{a}_{n}\right) \\
& \frac{\mathbf{v}_{n+1}-\mathbf{v}_{n}}{\Delta t}=\gamma \mathbf{a}_{n+1}+(1-\gamma) \mathbf{a}_{n}
\end{aligned}
$$

where

$$
\begin{aligned}
\alpha_{m} & =\frac{2 \rho_{\infty}-1}{\rho_{\infty}+1} & \alpha_{f} & =\frac{\rho_{\infty}}{\rho_{\infty}+1} \\
\beta & =\frac{1}{4}\left(1-\alpha_{m}+\alpha_{f}\right)^{2} & \gamma & =\frac{1}{2}-\alpha_{m}+\alpha_{f}
\end{aligned}
$$

Each parameter is a function of the spectral radius $\rho_{\infty}$ and the intervals through which they are valid can be determined based on $\rho_{\infty} \in[0,1]$. The spectral radius is a parameter that characterizes the convergence behaviour of the system, and the other Greek letter parameters can be thought of as numerical damping for the system and discretization error due to the discretization process.

Using the updated velocities and accelerations,

$$
\begin{aligned}
& \mathbf{v}_{n+1}\left(\mathbf{d}_{n+1}\right)=\frac{\gamma}{\beta \Delta t}\left(\mathbf{d}_{n+1}-\mathbf{d}_{n}\right)-\frac{\gamma-\beta}{\beta} \mathbf{v}_{n}-\frac{\gamma-2 \beta}{2 \beta} \Delta t \mathbf{a}_{n} \\
& \mathbf{a}_{n+1}\left(\mathbf{d}_{n+1}\right)=\frac{1}{\beta \Delta t^{2}}\left(\mathbf{d}_{n+1}-\mathbf{d}_{n}\right)-\frac{1}{\beta \Delta t} \mathbf{v}_{n}-\frac{1-2 \beta}{2 \beta} \mathbf{a}_{n}
\end{aligned}
$$

The Newmark methods generally search for equilibrium at the endpoints of the current time step, $t_{n}$ and $t_{n+1}$, but the generalized- $\alpha$ method shifts the evaluation to points between the endpoints at $t_{n+1-\alpha_{f}}$ and $t_{n+1-\alpha_{m}}$, respectively. The nonlinear 
equations of motion evaluated at the midpoints become

$$
\mathbf{M} \mathbf{a}_{n+1-\alpha_{m}}+\mathbf{f}_{i n t}\left(\mathbf{d}_{n+1-\alpha_{f}}\right)-\mathbf{f}_{e x t}\left(t_{n+1-\alpha_{f}}\right)=\mathbf{0}
$$

such that the midpoint displacements, velocities, and accelerations are linear combinations of the end points of the current time step as

$$
\left.\begin{array}{c}
\mathbf{a}_{n+1-\alpha_{m}}=\left(1-\alpha_{m}\right) \mathbf{a}_{n+1}+\alpha_{m} \mathbf{a}_{n} \\
\mathbf{v}_{n+1-\alpha_{f}}=\left(1-\alpha_{f}\right) \mathbf{v}_{n+1}+\alpha_{f} \mathbf{v}_{n} \\
\mathbf{d}_{n+1-\alpha_{f}}=\left(1-\alpha_{f}\right) \mathbf{d}_{n+1}+\alpha_{f} \mathbf{d}_{n} \\
\mathbf{f}_{e x t ; n+1-\alpha_{f}}=\left(1-\alpha_{f}\right) \mathbf{f}_{e x t ; n+1}+\alpha_{f} \mathbf{f}_{e x t ; n} .
\end{array}\right\}
$$

\subsubsection{Linearization and Iteration}

The equation of motion is nonlinear due to the geometric stiffness terms in the stressstrain relationship; therefore, root finding techniques involve iterative processes. A residual force vector is equal to the unbalanced forces from the nonlinear equations of motion, which is minimized upon each iteration until it is less than some pre-defined tolerance. The residual vector is the set of unbalanced forces that are greater than the tolerance. It is written as

$$
\mathbf{r}\left(\mathbf{d}_{n+1}\right)=\mathbf{M} \mathbf{a}_{n+1-\alpha_{m}}+\mathbf{f}_{i n t}\left(\mathbf{d}_{n+1-\alpha_{f}}\right)-\mathbf{f}_{e x t}\left(t_{n+1-\alpha_{f}}\right) \equiv 0
$$

and is normally defined as zero, since that is the objective. It is linearized at the endpoints by taking the series expansion

$$
\operatorname{Lin}(\mathbf{r})=\mathbf{r}\left(\mathbf{d}_{n+1}^{i}\right)+\left.\frac{\partial \mathbf{r}\left(\mathbf{d}_{n+1}\right)}{\partial \mathbf{d}_{n+1}}\right|^{i} \Delta \mathbf{d}_{n+1}^{i+1}
$$


where the higher order terms are neglected, being nonlinear on $\mathbf{d}$ themselves. The partial fraction term in the above equation is a matrix that comes from the Gateaux derivative of the residual equation. It represents the dynamic tangential stiffness at the current iteration and time-step end points. This stiffness matrix is

$$
\begin{aligned}
\mathbf{K}_{T_{\mathrm{dyn}}}\left(\mathbf{d}_{n+1}^{i}\right) & =\left.\frac{\partial \mathbf{r}\left(\mathbf{d}_{n+1}\right)}{\partial \mathbf{d}_{n+1}}\right|^{i} \\
& =\left.\left[\mathbf{M} \frac{\partial \mathbf{a}_{n+1-\alpha_{m}}}{\partial \mathbf{d}_{n+1}}+\frac{\partial \mathbf{f}_{i n t}\left(\mathbf{d}_{n+1-\alpha_{f}}\right)}{\partial \mathbf{d}_{n+1}}\right]\right|^{i} \\
& =\left.\left[\frac{1-\alpha_{m}}{\beta \Delta t^{2}} \mathbf{M}+\left(1-\alpha_{f}\right) \mathbf{K}_{T}\right]\right|^{i}
\end{aligned}
$$

where the dynamic tangential stiffness, $\mathbf{K}_{T_{\mathrm{dyn}}}$, is different from the tangential stiffness, $\mathbf{K}_{T}$. The tangential stiffness matrix is the partial derivative of the internal force vector, evaluated at the midpoints, with respect to the endpoint displacement, and is written as

$$
\mathbf{K}_{T}=\frac{\partial \mathbf{f}_{i n t}}{\partial \mathbf{d}_{n+1}}
$$

Therefore, using this definition for the dynamic tangential stiffness matrix, the Newton-Raphson incremental solution is given by

$$
\mathbf{K}_{T_{\mathrm{dyn}}}\left(\mathbf{d}_{n+1}^{i}\right) \Delta \mathbf{d}_{n+1}^{i+1}=-\mathbf{r}\left(\mathbf{d}_{n+1}^{i}\right)
$$

which implies the increment is

$$
\Delta \mathbf{d}_{n+1}^{i+1}=-\mathbf{K}_{T_{\mathrm{dyn}}^{-1}}\left(\mathbf{d}_{n+1}^{i}\right) \cdot \mathbf{r}\left(\mathbf{d}_{n+1}^{i}\right)
$$


Based on Eq. (2.78), the midpoint displacements, velocities, and accelerations update by the set of equations as follows:

$$
\begin{aligned}
& \Delta \mathbf{d}_{n+1-\alpha_{f}}^{i+1}=\frac{\partial \mathbf{d}_{n+1-\alpha_{f}}}{\partial \mathbf{d}_{n+1}} \Delta \mathbf{d}_{n+1}^{i+1}=\left(1-\alpha_{f}\right) \Delta \mathbf{d}_{n+1}^{i+1} \\
& \Delta \mathbf{v}_{n+1-\alpha_{f}}^{i+1}=\frac{\partial \mathbf{v}_{n+1-\alpha_{f}}}{\partial \mathbf{d}_{n+1}} \Delta \mathbf{d}_{n+1}^{i+1}=\frac{\left(1-\alpha_{f}\right) \gamma}{\beta \Delta t} \Delta \mathbf{d}_{n+1}^{i+1} \\
& \Delta \mathbf{a}_{n+1-\alpha_{m}}^{i+1}=\frac{\partial \mathbf{a}_{n+1-\alpha_{m}}}{\partial \mathbf{d}_{n+1}} \Delta \mathbf{d}_{n+1}^{i+1}=\frac{\left(1-\alpha_{m}\right)}{\beta \Delta t^{2}} \Delta \mathbf{d}_{n+1}^{i+1} .
\end{aligned}
$$

\subsection{Generalized- $\alpha$ Energy Momentum Method}

The term $f_{i n t}$ requires some extra attention: there are two ways of describing the internal force vector,

$$
\mathbf{f}_{i n t ; n+1-\alpha_{f}}=\mathbf{f}_{i n t}\left(\mathbf{u}_{n+1-\alpha_{f}}\right)
$$

or

$$
\mathbf{f}_{i n t ; n+1-\alpha_{f}}=\left(1-\alpha_{f}\right) \mathbf{f}_{i n t ; n+1}+\alpha_{f} \mathbf{f}_{i n t ; n}=\left(1-\alpha_{f}\right) \mathbf{f}_{i n t}\left(\mathbf{u}_{n+1}\right)+\alpha_{f} \mathbf{f}_{i n t}\left(\mathbf{u}_{n}\right)
$$

in which Eq. (2.80) is know as the implicit midpoint method, and Equation (2.81) is the trapezoid rule. So far, the analysis assumes the former; however, the endpoint strains could be substituted for the midpoint evaluation. If the strains in (2.80) are evaluated at the endpoints, such as

$$
\left.\mathbf{E}\left(\mathbf{u}_{n+1-\alpha_{f}}\right)\right|_{\alpha_{f}=\frac{1}{2}}=\frac{\mathbf{E}\left(\mathbf{u}_{n+1}\right)+\mathbf{E}\left(\mathbf{u}_{n}\right)}{2}
$$


then the exact Energy-Momentum Method by Simo and Tarnow [112] is recovered for Venant-Kirchhoff material. To control the higher-order elastic modes, and to generate a stable algorithm in the nonlinear regime, the endpoint-evaluated generalized- $\alpha$ method substitutes the midpoint-rule Green-Lagrange strain so that

$$
\begin{aligned}
\mathbf{E}\left(\mathbf{u}_{n+1-\alpha_{f}}\right) & =\left(1-\alpha_{f}\right) \mathbf{E}\left(\mathbf{u}_{n+1}\right)+\alpha_{f} \mathbf{E}\left(\mathbf{u}_{n}\right)+\xi\left(\mathbf{E}\left(\mathbf{u}_{n+1}\right)-\mathbf{E}\left(\mathbf{u}_{n}\right)\right) \\
& =\left(1-\alpha_{f}+\xi\right) \mathbf{E}\left(\mathbf{u}_{n+1}\right)+\left(\alpha_{f}-\xi\right) \mathbf{E}\left(\mathbf{u}_{n}\right)
\end{aligned}
$$

where $\xi$ represents an elastic damping term, which is always zero in the current computer model. Replacing the midpoint-evaluated strains in the generalized- $\alpha$ method with these strains yields the Generalized- $\alpha$ Energy Momentum Method (GEMM). The GEMM for a slender rod is studied in Kuhl and Crisfield [48].

The GEMM is based on the Energy-Momentum Method (EMM) developed by Simo and colleagues, see Refs. [112-114] for examples, which preserves energy and momentum exactly within each time step. However, due to convergence problems resulting from higher frequencies in stiff structural dynamics, some controllable numerical dissipation is necessary in these analyses. The original generalized- $\alpha$ method has the framework for such numerical dissipation, and as such is combined with the EMM for a recalculation of the internal strain vectors, evaluated at the endpoints, instead of at the midpoint.

To build the tangential stiffness matrix, the linearization proceeds as from Equation $(2.76)$ :

$$
\mathbf{K}_{T}=\frac{\partial \mathbf{f}_{i n t}}{\partial \mathbf{d}}=\mathrm{A}_{e=1}^{\text {nele }}\left[\int_{V^{(e)}}\left(\mathbf{B}_{l i n}^{\top} \mathbf{F}^{i} \mathbf{S}^{i}{ }_{\mathbf{d}}+\mathbf{B}_{l i n}^{\top} \mathbf{F}^{i}{ }_{\mathbf{d}} \mathbf{S}^{i}\right) \mathrm{dV}\right]
$$

where again, the comma denotes the derivative. The first term in the above equation 
represents the elastic and initial displacement stiffness, the second is the geometric stiffness. Both are functions of the deformation gradient, which are in turn functions of the nodal displacements. In the original generalized- $\alpha$ scheme, the derivative is with respect to the midpoint displacement. Here, the deformation gradient is modified so that it is a function of both endpoint displacements.

So, in the original,

$$
\begin{aligned}
\mathbf{E} & =\frac{1}{2}\left(\mathbf{F}^{\top} \mathbf{F}-\mathbf{1}\right) \\
\mathbf{E}, \mathbf{d} & =\frac{1}{2}\left(\mathbf{F}^{\top},{ }_{\mathbf{d}} \mathbf{F}+\mathbf{F}^{\top} \mathbf{F}, \mathbf{d}\right)=\mathbf{F B}_{l i n} .
\end{aligned}
$$

Because

$$
\mathbf{S}=\mathbf{C E}
$$

then

$$
\int_{V^{(e)}} \mathbf{B}_{l i n}^{\top} \mathbf{F}^{i} \mathbf{S}^{i}{ }_{\mathrm{d}} \mathrm{d} V=\int_{\mathrm{V}^{(e)}} \mathbf{B}_{\mathrm{lin}}^{\top} \mathbf{F}^{\mathrm{i}} \mathbf{C}\left(\mathbf{F}^{\mathrm{i}}\right)^{\top} \mathbf{B}_{\mathrm{lin}} \mathrm{dV}
$$

for the elastic displacement stiffness. The geometric stiffness matrix is

$$
\int_{V^{(e)}} \mathbf{B}_{l i n}^{\top} \mathbf{F}^{i}, \mathbf{d}^{i} \mathbf{S}^{\mathrm{d} V}=\int_{\mathrm{V}^{(\mathrm{e})}} \mathbf{B}_{\mathrm{lin}}^{\top} \mathbf{S}^{\mathrm{i}} \mathbf{B}_{\mathrm{lin}} \mathrm{dV}
$$

where $\mathbf{S}^{i}$ is in Voigt-matrix form, and all the displacement dependencies, $\mathbf{d}=\mathbf{d}_{n+1-\alpha_{f}}$, such that

$$
\mathbf{K}_{T}=\int_{V^{(e)}} \mathbf{B}_{l i n}^{\top} \mathbf{F}^{i} \mathbf{C}\left(\mathbf{F}^{i}\right)^{\top} \mathbf{B}_{l i n} \mathrm{dV}+\int_{\mathrm{V}^{(e)}} \mathbf{B}_{\operatorname{lin}}^{\top} \mathbf{S}^{\mathrm{i}} \mathbf{B}_{\text {lin }} \mathrm{dV}
$$


However, now the strain is a function of both the previous time-step displacements and the current time displacements. Using the theory from Kuhl and Crisfield and a technical note from the Technical University of Munich [110], the internal force vector is

$$
\mathbf{f}_{i n t_{m}}=\int_{V^{(e)}} \mathbf{B}_{l i n} \mathbf{F}_{m} \mathbf{S}_{m} \mathrm{dV}
$$

where the midpoint deformation gradient and stress are

$$
\begin{aligned}
& \mathbf{F}_{m}=\left(1-\alpha_{f}\right) \mathbf{F}_{n+1}+\alpha_{f} \mathbf{F}_{n} \\
& \mathbf{S}_{m}=\left(1-\alpha_{f}+\xi\right) \mathbf{C E}_{n+1}+\left(\alpha_{f}-\xi\right) \mathbf{C E}_{n}
\end{aligned}
$$

The tangential stiffness matrix is still the derivative of the internal force vector with respect to the displacement,

$$
\begin{aligned}
\mathbf{K}_{T} & =\frac{\partial \mathbf{f}_{i n t}}{\partial \mathbf{d}_{n+1}} \\
& =\int_{V^{(e)}} \mathbf{B}_{l i n} \mathbf{F}_{m} \mathbf{S}_{m, \mathbf{d}} \mathrm{dV}+\int_{\mathrm{V}^{(e)}} \mathbf{B}_{\operatorname{lin}} \mathbf{F}_{\mathrm{m}, \mathbf{d}} \mathbf{S}_{\mathrm{m}} \mathrm{dV} \\
& =\left.\int_{V^{(e)}}\left(1-\alpha_{f}+\xi\right) \mathbf{B}_{l i n} \mathbf{F}_{m} \mathbf{S}_{m, \mathbf{d}}\right|_{n+1} \mathrm{dV}+\left.\int_{\mathrm{V}^{(e)}}(\alpha-\xi) \mathbf{B}_{l i n} \mathbf{F}_{\mathrm{m}, \mathbf{d}}\right|_{\mathrm{n}+1} \mathbf{S}_{\mathrm{m}} \mathrm{dV} \\
& =\underbrace{\int_{V^{(e)}}(1-\alpha+\xi) \mathbf{B}_{m}^{\mathrm{T}} \mathbf{C B}_{n} \mathrm{dV}}_{K_{e u}}+\underbrace{\int_{V^{(e)}}(1-\alpha) \mathbf{B}_{l i n}^{\mathrm{T}} \mathbf{S}_{m} \mathbf{B}_{l i n} \mathrm{dV}}_{K_{g}}
\end{aligned}
$$

where

$$
\mathbf{B}_{m}=\mathbf{F}_{m}^{\top} \mathbf{B}_{l i n}
$$

Here, the material stiffness matrix, $\mathbf{K}_{e u}$, is asymmetric because of the product of $\mathbf{B}_{m}$ 
and $\mathbf{B}_{n}$. The geometric stiffness $\mathbf{K}_{g}$ remains symmetric. 


\section{Chapter 3}

\section{Multibody Dynamics}

In this Chapter, the articulation of the helicopter hub is examined similarly to the methods common in, but not exclusive to, robotics. The Denavit-Hartenberg model for kinematic analysis is well established and trusted for robotic systems. The same kinematic concept applies here, as the hub articulation can be modelled as a robotic system with the rotor blade replacing the robot hand (or manipulator). Typical texts examine open chain kinematics, and books such as Niku [49] or Sciavicco and Siciliano [50] are excellent initial sources.

Closed kinematic chains are a bit more difficult to analyse in robot kinematics, due to the static indeterminacy of the system. However, virtually cutting the kinematic tree to create two open chains, such as in Luh and Zheng [65], enable one to solve the system with geometric constraints. Additionally, there is another chain that describes the motion of the adaptive pitch controller mass, which is only dynamically coupled with the control chain, and is therefore treated as an open chain. 


\subsection{Modelling the Position and Orientation of Joints in Open Chains}

Often transformations between co-ordinate frames are represented by a 3-by-3 orthonormal rotation matrix, which contains the rotational changes between the frames. Such transformations require a position vector, described from the inertial frame or from the end frame, to describe the location of the end point. Together the rotation matrix and position vector describe the orientation and position of the end effector. However, it is desirable to have both position and orientation described within one co-ordinate transformation, as opposed to having separate descriptions for position and orientation individually. Therefore, with reference to Figure 3.1, consider a point in space, $P$.

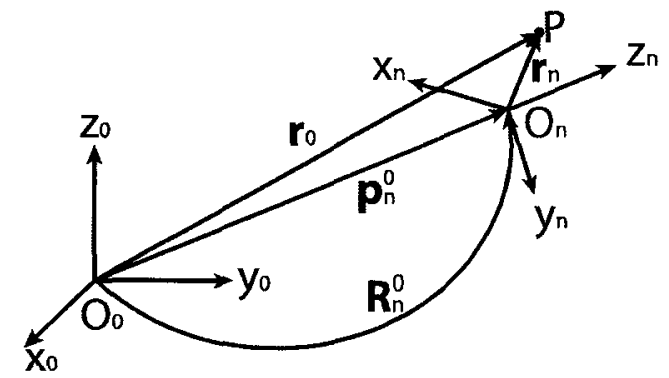

Figure 3.1: Position and orientation transformation between inertial and end frames.

As shown in the Figure, $\mathbf{r}_{0}$ represents Point $\mathrm{P}$ in the initial frame of reference, likewise $\mathbf{r}_{n}$ represents the Point in the Frame $O_{n}$. If $\mathbf{p}_{n}^{0}$ represents the vector describing the origin of Frame $O_{n}$ with respect to the inertial frame $O_{0}$, and $\mathbf{R}_{n}^{0}$ is the rotation matrix between the two frames, then $P$ with respect to the origin is

$$
\mathbf{r}_{0}=\mathbf{p}_{n}^{0}+\mathbf{R}_{n}^{0} \mathbf{r}_{n}
$$


The transformation from Frame $O_{0}$ to Frame $O_{n}$ is $\mathbf{R}_{n}^{0}$. If the generic vector $\mathbf{r}$ is augmented by a fourth scaling component as in

$$
\mathbf{r}^{+}=\left[\begin{array}{l}
\mathbf{r} \\
1
\end{array}\right]
$$

then using Equations (3.2) in (3.1) produces

$$
\mathbf{r}_{0}^{+}=\mathbf{A}_{n}^{0} \mathbf{r}_{n}^{+}
$$

where

$$
\mathbf{A}_{n}^{0}=\left[\begin{array}{cc}
\mathbf{R}_{n}^{0} & \mathbf{p}_{n}^{0} \\
\mathbf{0} & 1
\end{array}\right]
$$

Now, $\mathbf{r}^{+}$is the homogeneous representation of a position vector and $\mathbf{A}_{n}^{0}$ is the augmented rotation-position matrix, or the homogeneous transformation matrix. Clearly, the inverse of matrix $\mathbf{A}_{n}^{0}$ is not its transpose, because it is no longer orthogonal. However, a compact form of the position and orientation transformations between any two frames of reference is satisfied. From this point onward, $\mathbf{r}$ will be used in place of $\mathbf{r}^{+}$ to denote the augmented position vector unless specified otherwise.

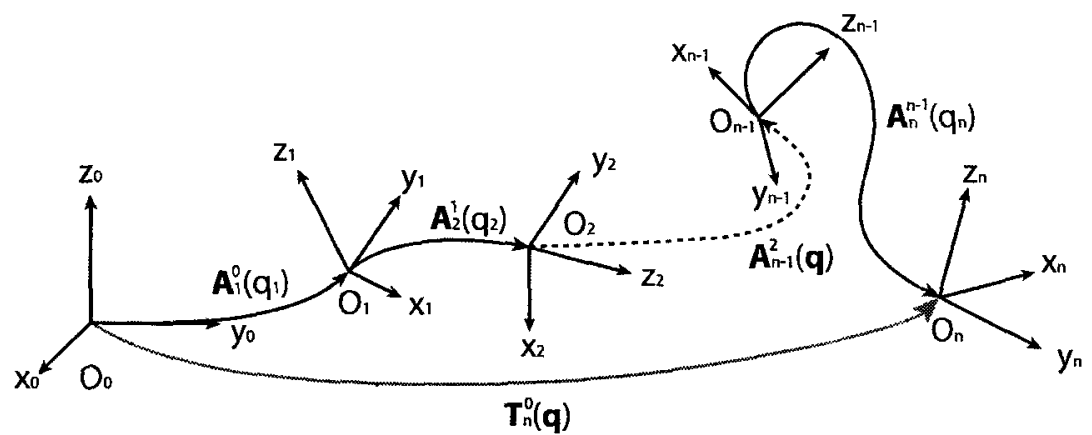

Figure 3.2: Co-ordinate transformations in an open kinematic chain. 
Open kinematic chains can be described using homogeneous transformations by supposing that each link in the chain relates its current frame of reference to the next one. Figure 3.2 shows an open chain manipulator represented by co-ordinate systems. Referring to the figure, each joint connects two consecutive links, so the relationship between each co-ordinate frame is dependent on the orientation of the joint and the linkage. Labelling each joint and each link, the relationships between consecutive links can be recursively determined. For example, Joint 2, denoted by the frame at $O_{1}$, connects Link 0 and Link 1 , through the transformation $\mathbf{A}_{1}^{0}\left(q_{1}\right)$. Therefore, the construction of a relationship between the inertial point and the end-effector, which is in the helicopter case the rotor blade, is a multiplication of co-ordinate transformation systems. That means that the position and orientation between the end frame with respect to Frame 0 is

$$
\mathbf{T}_{n}^{0}(\mathbf{q})=\mathbf{A}_{1}^{0}\left(q_{1}\right) \mathbf{A}_{2}^{1}\left(q_{2}\right) \cdots \mathbf{A}_{n}^{n-1}\left(q_{n}\right)
$$

in which the transformation of each co-ordinate frame to the next, $\mathbf{A}_{i}^{i-1}\left(q_{i}\right)$ is only dependent on a single joint variable, $q_{i}$.

Furthermore, because it is common to denote the end effector orientation with three basis vectors, that notation is adopted in this dissertation. Typically,

$$
\mathbf{T}_{n}^{0}(\mathbf{q})=\left[\begin{array}{llll}
\mathbf{n} & \mathbf{o} & \mathbf{a} & \mathbf{p} \\
0 & 0 & 0 & 1
\end{array}\right]
$$

such that $\mathbf{a}$ is approach vector, in the direction of the end-effector, $\mathbf{o}$ is the orientation vector, normal to the approach vector, which describes the orientation of the endeffector, and $\mathbf{n}$ is the normal vector, orthogonal to the previous two vectors completing the right-hand rule. The vector $\mathbf{p}$ is still the position vector, which describes the position of the origin of the final frame of reference with respect to the base. In the 
multibody dynamics within this thesis, $\mathbf{p}$ denotes the position of frames with respect to one-another, $\mathbf{r}$ normally describes the position of a point with respect to a given frame.

\subsubsection{Denavit-Hartenberg Representation of Forward Kine- matics}

A geometric modelling technique developed by Denavit and Hartenberg [115] was established for lower-pair joints that applies to any mechanism, independent of complexity, joint combinations, or joint order. The Denavit-Hartenberg (DH) representation works for both prismatic and revolute joints, although there are a few differences between them, which are the two types of one degree of freedom joints studied in this thesis.

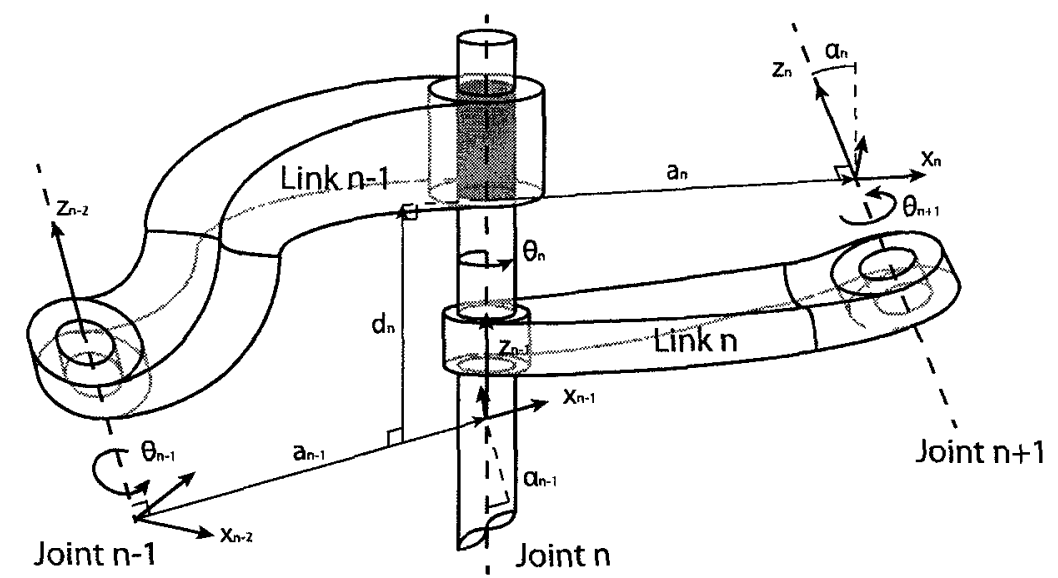

Figure 3.3: Three joints showing frames of reference and transformation parameters.

Figure 3.3 depicts three Joints $(n-1, n, n+1)$ with two links that represent any general arrangement in a robot or helicopter hub. All joints are represented by a $z$-axis, and each joint's $z$-axis is labelled one less than the joint number. If the joint is revolute, the $z$-axis is in the direction of rotation using the right-hand rule to 
describe positive rotations. In the Figure, Joint $n-1$ has a rotary axis at $z_{n-2}$, with the joint variable $\theta_{n-1}$, indicating the positive rotation. If the joint is prismatic, the $z$-axis is along the direction of positive linear motion, as shown by Joint $n$ with the $z_{n-1}$ in the direction of translation and $d_{n}$ as the joint variable.

Successive joints' $z$-axes may be skewed rather than intersecting or parallel, which means their $z$-axes have a twist angle $\alpha$ offset. In such a case, the shortest distance between two consecutive $z$-axes is a common normal perpendicular to both skew lines, and the $x$-axis always represents that common normal. As referenced in the Figure, the offset distance, $a_{n-1}$, is the common normal between Joints $n-1$ and $n$, and $a_{n}$ is the common normal between Joints $n$ and $n+1$. There are two special cases for common normal offsets: if successive joints are parallel or they are intersecting. If two $z$-axes are parallel, then there are an infinite number of common normals, so the antecedent normal direction is collinear with the precedent. If they are intersecting, the $x$-axis is chosen perpendicular to the plane created by the two $z$-axes.

So, in summary, for revolute joints $\theta_{n}$ is the joint variable, representing a rotation about the $z_{n-1}$-axis, and for prismatic joints $d_{n}$ represents the translation along the $z_{n-1}$-axis. The length of each common normal is represented by $a_{n}$, and the angle between the $z_{n^{-}}$and $z_{n+1}$-axes is $\alpha_{n}$.

Once the DH parameters are labelled, two sets of transformations are necessary to describe the relation between co-ordinate frames. The first set requires a rotation about the $z_{n}$-axis through an angle $\theta_{n+1}$ and then a translation along the $z_{n}$-axis for a distance of $d_{n+1}$. The rotation makes the $x_{n^{-}}$and $x_{n+1}$-axes parallel to one another, and the translation makes them coincident. The second set of transformations requires a translation of $a_{n+1}$ from the $x_{n}$-axis to the $x_{n+1}$-axis, followed by a rotation about the $x_{n+1}$-axis. The translation brings the origins of the two co-ordinate frames together, and the rotation causes them to be identical. Using matrices, this is 


$$
\begin{aligned}
\mathbf{T}_{n}^{n-1}=\mathbf{A}_{n}= & {\left[\begin{array}{cccc}
\cos \theta_{n} & -\sin \theta_{n} & 0 & 0 \\
\sin \theta_{n} & \cos \theta_{n} & 0 & 0 \\
0 & 0 & 1 & d_{n} \\
0 & 0 & 0 & 1
\end{array}\right] \times } \\
& {\left[\begin{array}{cccc}
1 & 0 & 0 & a_{n} \\
0 & \cos \alpha_{n} & -\sin \alpha_{n} & 0 \\
0 & \sin \alpha_{n} & \cos \alpha_{n} & 0 \\
0 & 0 & 0 & 1
\end{array}\right] } \\
= & {\left[\begin{array}{cccc}
\cos \theta_{n} & -\sin \theta_{n} \cos \alpha_{n} & \sin \theta_{n} \sin \alpha_{n} & a_{n} \cos \theta_{n} \\
\sin \theta_{n} & \cos \theta_{n} \cos \alpha_{n} & -\cos \theta_{n} \sin \alpha_{n} & a_{n} \sin \theta_{n} \\
0 & \sin \alpha_{n} & \cos \alpha_{n} & d_{n} \\
0 & 0 & 0 & 1
\end{array}\right] . }
\end{aligned}
$$

$\mathbf{A}_{n}$ represents the homogeneous transformation matrix, with its columns representing the three vector orientations and position of the next frame with respect to Frame $n$.

\subsection{Lagrangian Mechanics for a Multiple Degrees of Freedom Dynamic System}

Combining the kinetic and potential energies of the chains to describe the system Lagrangian and using the Lagrange equations creates the system equations of motion. The Lagrangian is

$$
L=K-V
$$

where $K$ is the kinetic energy and $V$ is the potential energy. 


\section{Kinetic energy}

The kinetic energy of the system is

$$
\begin{aligned}
K & =\frac{1}{2} \mathbf{v}^{\top} m_{G} \mathbf{v}+\frac{1}{2} \boldsymbol{\omega} \mathbf{h}_{G} \\
& =\frac{1}{2} \mathbf{v}^{\top} m_{G} \mathbf{v}+\frac{1}{2} \boldsymbol{\omega}^{\boldsymbol{\top}} \mathbf{I}_{G} \boldsymbol{\omega},
\end{aligned}
$$

in which $\mathbf{v}$ is the generalized velocity vector, and $\mathbf{h}$ is the angular momentum vector, corresponding with the indices for each link. Evaluating this equation requires the velocity for a rigid body at the centre of mass, $G$, as well as the moments of inertia $\mathbf{I}_{G}$.

The transformation matrix between the inertial frame of reference and the end co-ordinates is already known, from multiplying the $\mathbf{A}_{n}$ matrices consecutively, and since the velocities are the time derivatives of the positions, one possible solution takes advantage of recursive derivatives. For any $\mathbf{A}_{n}$, the derivative with respect to a revolute variable $\theta_{n}$, is

$$
\begin{aligned}
\frac{\partial \mathbf{A}_{n}}{\partial \theta_{n}} & =\frac{\partial}{\partial \theta_{n}}\left[\begin{array}{cccc}
\cos \theta_{n} & -\sin \theta_{n} \cos \alpha_{n} & \sin \theta_{n} \sin \alpha_{n} & a_{n} \cos \theta_{n} \\
\sin \theta_{n} & \cos \theta_{n} \cos \alpha_{n} & -\cos \theta_{n} \sin \alpha_{n} & a_{n} \sin \theta_{n} \\
0 & \sin \alpha_{n} & \cos \alpha_{n} & d_{n} \\
0 & 0 & 0 & 1
\end{array}\right] \\
& =\left[\begin{array}{cccc}
-\sin \theta_{n} & -\cos \theta_{n} \cos \alpha_{n} & \cos \theta_{n} \sin \alpha_{n} & -a_{n} \sin \theta_{n} \\
\cos \theta_{n} & -\sin \theta_{n} \cos \alpha_{n} & \sin \theta_{n} \sin \alpha_{n} & a_{n} \cos \theta_{n} \\
0 & 0 & 0 & 0 \\
0 & 0 & 0 & 0
\end{array}\right] .
\end{aligned}
$$

The above is equivalent to

$$
\frac{\partial \mathbf{A}_{n}}{\partial \theta_{n}}=\mathbf{Q}_{n} \mathbf{A}_{n},
$$


where the matrix $\mathbf{Q}_{n}$ multiplies the transformation matrix to yield the appropriate derivative, written explicitly as

$$
\mathbf{Q}_{n}=\left[\begin{array}{cccc}
0 & -1 & 0 & 0 \\
1 & 0 & 0 & 0 \\
0 & 0 & 0 & 0 \\
0 & 0 & 0 & 0
\end{array}\right]
$$

The same matrix derivative applies for a prismatic joint, except that the derivative is

$$
\frac{\partial \mathbf{A}_{n}}{\partial \mathbf{d}_{n}}=\left[\begin{array}{cccc}
0 & 0 & 0 & 0 \\
0 & 0 & 0 & 0 \\
0 & 0 & 0 & d_{n} \\
0 & 0 & 0 & 0
\end{array}\right]=\mathbf{Q}_{n} \mathbf{A}_{n}
$$

so

$$
\mathbf{Q}_{n}=\left[\begin{array}{llll}
0 & 0 & 0 & 0 \\
0 & 0 & 0 & 0 \\
0 & 0 & 0 & 1 \\
0 & 0 & 0 & 0
\end{array}\right]
$$

Therefore, derivatives of $\mathbf{T}_{n}^{0}$ look like

$$
\mathbf{U}_{\imath \jmath}=\frac{\partial \mathbf{T}_{\imath}^{0}}{\partial q_{\jmath}}=\frac{\partial\left(\prod_{k=1}^{i} \mathbf{A}_{k}\right)}{\partial q_{\jmath}}=\mathbf{A}_{1} \mathbf{A}_{2} \cdots \mathbf{Q}_{\jmath} \mathbf{A}_{\jmath} \cdots \mathbf{A}_{\imath}, \quad \text { for } j \leq i
$$

Higher derivatives (up to second order will be required for the Coriolis and centripetal accelerations) can be found similarly by taking the derivatives twice:

$$
\begin{aligned}
\mathbf{U}_{i \jmath k} & =\frac{\partial \mathbf{U}_{i \jmath}}{\partial q_{k}}, \quad \quad \text { for } \jmath, k \leq i \\
& =\mathbf{A}_{1} \mathbf{A}_{2} \cdots \mathbf{Q}_{\jmath} \mathbf{A}_{\jmath} \cdots \mathbf{Q}_{k} \mathbf{A}_{k} \cdots \mathbf{A}_{\imath}
\end{aligned}
$$


Because the kinetic energy involves the velocities, the position of a point $\mathbf{p}_{\imath}$, on any of the links must be determined.

$$
\mathbf{p}_{\imath}=\mathbf{T}_{\imath}^{0} \mathbf{r}_{\imath}
$$

where $\mathbf{r}_{\imath}$ is a fixed point on the rigid link, and thus, it is not a function of time. The velocity of the point is dependent on the velocities of the preceding links, such that

$$
\mathbf{v}_{\imath}=\dot{\mathbf{p}}_{\imath}=\frac{d}{d t}\left(\mathbf{T}_{\imath}^{0} \mathbf{r}_{\imath}\right)=\sum_{\jmath=1}^{\imath}\left(\frac{\partial\left(\mathbf{T}_{\imath}^{0}\right)}{\partial q_{\jmath}} \frac{d q_{\jmath}}{d t}\right) \mathbf{r}_{\imath}=\sum_{\jmath=1}^{\imath}\left(\mathbf{U}_{\imath \jmath} \dot{q}_{\jmath}\right) \cdot \mathbf{r}_{\imath}
$$

The kinetic energy of an infinitesimal mass $d m_{\imath}$, at point $\mathbf{p}_{\imath}$ on link $\imath$, is

$$
d K_{\imath}=\frac{1}{2}\left(\dot{x}_{\imath}^{2}+\dot{y}_{\imath}^{2}+\dot{z}_{\imath}^{2}\right) d m
$$

which when combined with Equation (3.17), results in

$$
d K_{\imath}=\frac{1}{2}\left[\left(\sum_{\jmath=1}^{\imath}\left(\mathbf{U}_{\imath \jmath} \dot{q}_{\jmath}\right) \cdot \mathbf{r}_{\imath}\right) \cdot\left(\sum_{k=1}^{\imath}\left(\mathbf{U}_{\imath k} \dot{q}_{k}\right) \cdot \mathbf{r}_{\imath}\right)\right] d m_{\imath}
$$

where $\jmath$ and $k$ represent the different joint numbers. From this,

$$
\begin{aligned}
K_{\imath}=\int_{n} d K_{\imath} & =\frac{1}{2}\left[\sum_{\jmath=1}^{\imath} \sum_{k=1}^{\imath} \mathbf{U}_{\imath \jmath} \cdot\left(\int_{n} \mathbf{r}_{\imath} \mathbf{r}_{\imath}^{\top} d m_{\imath}\right) \cdot \mathbf{U}_{\imath k}^{\top} \dot{q}_{\jmath} \dot{q}_{k}\right] \\
& =\frac{1}{2} \sum_{\jmath=1}^{\imath} \sum_{k=1}^{\imath} \mathbf{U}_{\imath \jmath} \cdot \mathbf{J}_{\imath} \cdot \mathbf{U}_{\imath k}^{\top} \dot{q}_{\jmath} \dot{q}_{k},
\end{aligned}
$$

in which the pseudo-inertia matrix $\mathbf{J}_{\imath}$, is given as 


$$
\begin{aligned}
\mathbf{J}_{\imath} & =\int_{n} \mathbf{r}_{\imath} \mathbf{r}_{\imath}^{\top} d m_{\imath} \\
& =\left[\begin{array}{cccc}
\frac{-I_{x x}+I_{y y}+I_{z z}}{2} & I_{x y} & I_{x z} & m_{\imath} \bar{x}_{\imath} \\
I_{x y} & \frac{I_{x x}-I_{y y}+I_{z z}}{2} & I_{y z} & m_{\imath} \bar{y}_{\imath} \\
I_{x z} & I_{y z} & \frac{I_{x x}+I_{y y}-I_{z z}}{2} & m_{\imath} \bar{z}_{\imath} \\
m_{\imath} \bar{x}_{\imath} & m_{\imath} \bar{y}_{\imath} & m_{\imath} \bar{z}_{\imath} & m_{\imath}
\end{array}\right] .
\end{aligned}
$$

Thus, the total kinetic energy for the entire system is

$$
\begin{aligned}
K & =\sum_{\imath=1}^{n} K_{\imath} \\
& =\frac{1}{2} \sum_{\imath=1}^{n} \sum_{\jmath=1}^{\imath} \sum_{k=1}^{\imath} \mathrm{U}_{\imath \jmath} \cdot \mathbf{J}_{\imath} \cdot \mathbf{U}_{\imath k}^{\top} \dot{q}_{\jmath} \dot{q}_{k}
\end{aligned}
$$

\section{Potential energy}

The total potential energy is the sum of the potential energies of each link in the system, and is written as

$$
\begin{aligned}
V=\sum_{\imath=1}^{n} V_{\imath} & =\sum_{\imath=1}^{n}\left(m_{\imath} \mathbf{g} \cdot \mathbf{p}_{\imath}\right)+\sum_{\imath=1}^{n}\left(\frac{1}{2} \mathbf{k}\left(\mathbf{q}_{\imath}-\mathbf{q}_{\imath_{0}}\right)^{2}\right) \\
& =\sum_{\imath=1}^{n}\left(m_{\imath} \mathbf{g} \cdot\left(\mathbf{T}_{\imath}^{0} \mathbf{r}_{G_{\imath}}\right)\right)+\sum_{\imath=1}^{n}\left(\frac{1}{2} \mathbf{k}\left(\mathbf{q}_{\imath}-\mathbf{q}_{\imath 0}\right)^{2}\right)
\end{aligned}
$$

in which gravity is $\mathbf{g}=\left[\begin{array}{llll}g_{x} & g_{y} & g_{z} & 0\end{array}\right]^{\top}$, and $\mathbf{r}_{G_{2}}$ is the location of the centre of mass for the link, relative to the frame representing the link. The second term in these equations represents hinge springs on each joint; the $\mathbf{q}_{\imath}$ variable is the current position of the joint hinge, and $\mathbf{q}_{\imath_{0}}$ is the equilibrium position of the joint. 
Therefore, the Lagrangian is given as

$$
L=K-V=\frac{1}{2} \sum_{\imath=1}^{n} \sum_{\jmath=1}^{\imath} \sum_{k=1}^{\imath} \mathbf{U}_{\imath \jmath} \mathbf{J}_{\imath} \mathbf{U}_{\imath k}^{\top} \dot{q}_{\jmath} \dot{q}_{k}-\sum_{\imath=1}^{n}\left(m_{\imath} \mathbf{g} \cdot\left(\mathbf{T}_{\imath}^{0} \mathbf{r}_{G_{\imath}}\right)+\frac{1}{2} \mathbf{k}\left(\mathbf{q}_{\imath}-\mathbf{q}_{\imath_{0}}\right)^{2}\right)
$$

The hinge stiffness terms, denoted by $k_{\imath}$, act only on the individual hinge displacement from its equilibrium position. Therefore, differentiation on the

$$
\frac{1}{2} \mathbf{k}\left(\mathbf{q}_{2}-\mathbf{q}_{2_{0}}\right)^{2}
$$

term is zero for all variables not $\mathbf{q}_{2}$. With this in mind, differentiating the Lagrangian results in the dynamic equations of motion, which for a general, multi-axis robot, is

$$
\boldsymbol{\tau}=\sum_{\jmath=1}^{n} \mathbf{D}_{\imath \jmath} \ddot{\mathbf{q}}_{\jmath}+\sum_{\jmath=1}^{n} \sum_{k=1}^{n} \mathbf{D}_{\imath \jmath} \dot{\mathbf{q}}_{\jmath} \dot{\mathrm{q}}_{k}+\mathbf{K q}+\mathbf{f}_{g}
$$

where

$$
\begin{gathered}
\mathbf{D}_{\imath \jmath}=\sum_{p=\max (\imath, \jmath)}^{n} \mathbf{U}_{p \jmath} \mathbf{J}_{p} \mathbf{U}_{p \imath}^{\top} \\
\mathbf{D}_{\imath \jmath k}=\sum_{p=\max (\imath, \jmath, k)}^{n} \mathbf{U}_{p \jmath k} \mathbf{J}_{p} \mathbf{U}_{p i}^{\top} \\
\mathbf{f}_{g}=\sum_{p=\max (\imath, \jmath)}^{n} m_{p} \mathbf{g}^{\top} \mathbf{U}_{p \imath} \overline{\mathbf{r}}_{\imath}
\end{gathered}
$$

and $\mathbf{K}$ is a matrix of stiffness values according to the joint variables These are the explicit representations of the angular acceleration inertial terms in Eq. (3.25a), the Coriolis and centripetal torques in Eq. (3.25b), the gravity terms in Eq. (3.25c), and the inter-mass spring forces, $\mathbf{k}$. 


\subsection{Closed Chain Kinematics with Holonomic Constraints}

Closed kinematic chains are treated much in the same manner as open chains, in that they can be analysed as branch structures within the mechanism's kinematic tree. However, at some joint, the motion splits into two branches, for example, as shown by Joint 2 at $O_{1}$, in Figure 3.4. At the split joint, the branches are labelled with primes to denote which path, or which set of transformations, the links are described by. Each branch has independent descriptions of the motion, but because the branches join again at some co-ordinate frame, in this case, $O_{n-1}$, there are constraints on the end position and orientation of each chain.

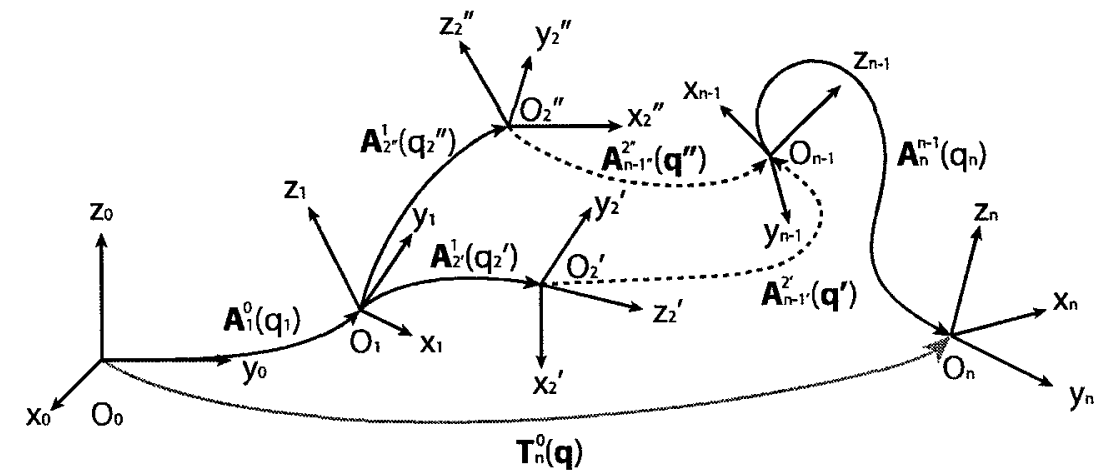

Figure 3.4: Diagram of a general closed chain kinematic mechanism.

Similar to Luh and Zheng [65], a joint in the closed kinematic chain is cut to create an open tree structure with kinematic chains as branches. In the Figure, this could be any joint, although usually an unactuated one, within the loop with the $\mathbf{q}^{\prime}$ or $\mathbf{q}^{\prime \prime}$ variables. Each branch is analysed as an open chain system, with the DH representation as normal. For each loop the position and orientation of the frames 
are

$$
\mathbf{A}_{n}^{1}\left(\mathbf{q}^{\prime}\right)=\mathbf{A}_{2^{\prime}}^{1}\left(\mathbf{q}_{2^{\prime}}\right) \cdots \mathbf{A}_{n}^{n-1^{\prime}}\left(\mathbf{q}_{n^{\prime}}\right)
$$

and

$$
\mathbf{A}_{n}^{1}\left(\mathbf{q}^{\prime \prime}\right)=\mathbf{A}_{2^{\prime \prime}}^{1}\left(\mathbf{q}_{2^{\prime \prime}}\right) \cdots \mathbf{A}_{n}^{n-1^{\prime \prime}}\left(\mathbf{q}_{n^{\prime \prime}}\right)
$$

where $\mathbf{q}^{\prime}$ and $\mathbf{q}^{\prime \prime}$ are the joint variables in each chain respectively. Normally, the links connected to each other through Joint $n-1$ need to be aligned in such a way that the Links $n-2$ in each branch must have colinear $z$-axes. Therefore, three orientation constraints are

$$
\mathbf{z}_{n-1}^{n-2}\left(\mathbf{q}^{\prime}\right)=\mathbf{z}_{n-1}^{n-2}\left(\mathbf{q}^{\prime \prime}\right)
$$

which can be referenced to the separation link, or preferably to the inertial frame of reference, by

$$
\mathbf{z}_{n-2}^{0}\left(\mathbf{q}^{\prime}\right)=\mathbf{z}_{n-2}^{0}\left(\mathbf{q}^{\prime \prime}\right)
$$

If the cut joint is prismatic, then a further constraint is that the angle between the previous two links is fixed, so similar to the $z$-axes,

$$
\mathbf{x}_{n-2}^{i \top}\left(\mathbf{q}^{\prime}\right) \mathbf{x}_{n-2}^{i}\left(\mathbf{q}^{\prime \prime}\right)=\cos \theta
$$

and there is no requirement to fix the $y$-axes, because they already automatically satisfy the right-hand rule, providing no further constraint. 
The positional constraint of the cut joint is given by

$$
\mathbf{R}_{n-2}^{i}\left(\mathbf{q}^{\prime}\right)\left(\mathbf{p}_{n-2}^{i}\left(\mathbf{q}^{\prime}\right)-\mathbf{p}_{n-2}^{i}\left(\mathbf{q}^{\prime \prime}\right)\right)=\left[\begin{array}{lll}
0 & 0 & d
\end{array}\right]^{\top}
$$

However, if the cut joint is prismatic, then only the first two equalities of Equation (3.31) hold, because the offset on the $z$-axis is variable.

In summary, if the virtually cut joint is revolute the six constraints are:

$$
\begin{array}{r}
\mathbf{R}_{n-2}^{i}\left(\mathbf{q}^{\prime}\right)\left(\mathbf{p}_{n-2}^{i}\left(\mathbf{q}^{\prime}\right)-\mathbf{p}_{n-2}^{i}\left(\mathbf{q}^{\prime \prime}\right)\right)=\left[\begin{array}{lll}
0 & 0 & d
\end{array}\right]^{\top} \\
\mathbf{z}_{n-2}^{0}\left(\mathbf{q}^{\prime}\right)=\mathbf{z}_{n-2}^{0}\left(\mathbf{q}^{\prime \prime}\right)
\end{array}
$$

If the virtually cut joint is prismatic, then the six constraints are:

$$
\begin{array}{r}
{\left[\mathbf{x}_{n-2}^{i \top}\left(\mathbf{q}^{\prime}\right) \mathbf{y}_{n-2}^{i \top}\left(\mathbf{q}^{\prime}\right)\right]\left(\mathbf{p}_{n-2}^{i}\left(\mathbf{q}^{\prime}\right)-\mathbf{p}_{n-2}^{i}\left(\mathbf{q}^{\prime \prime}\right)\right)=\left[\begin{array}{ll}
0 & 0
\end{array}\right]^{\top}} \\
\mathbf{z}_{n-1}^{0}\left(\mathbf{q}^{\prime}\right)=\mathbf{z}_{n-1}^{0}\left(\mathbf{q}^{\prime \prime}\right) \\
\mathbf{x}_{n-2}^{i \top}\left(\mathbf{q}^{\prime}\right) \mathbf{x}_{n-2}^{i}\left(\mathbf{q}^{\prime \prime}\right)=\cos \theta .
\end{array}
$$

Finally, the rigid body mechanics of the articulated helicopter hub are completely described using the Denavit-Hartenberg representation. The next section details the combination of the chains, the aerodynamic and control branches, without explicitly writing them out, because they are unwieldy when written symbolically and would be nonsensical in their trigonometric form.

\subsection{Modelling the Rotor Hub Mechanics}

From the Introduction, Figure 1.5 shows an example of the helicopter hub articulation. Using that figure as a basis, axes and frames of reference are applied to appropriate 
points, mainly the joints, as shown in Figure 3.5, in the way mentioned in the previous section to create a compatible DH model. For clarity, the joint variables are shown overlaid on the articulation in Figure 3.6.

Following the methodology described in Reference [49], for this system of coordinates, the DH parameters are as follows in Tables 3.1-3.2.

Table 3.2: Control Chain and APL DoF

Table 3.1: Aerodynamic Chain

\begin{tabular}{ccccc}
\hline Link \# & $\theta_{n}$ & $d_{n}$ & $a_{n}$ & $\alpha_{n}$ \\
\hline $\mathrm{A} 1$ & $\psi$ & 0 & $H$ & $\frac{\pi}{2}$ \\
$\mathrm{~A} 2$ & $\beta$ & 0 & $F$ & $-\frac{\pi}{2}$ \\
$\mathrm{~A} 3$ & $\zeta+\frac{\pi}{2}$ & 0 & 0 & $\frac{\pi}{2}$ \\
$\mathrm{~A} 4$ & $\theta$ & 0 & 0 & $-\frac{\pi}{2}$ \\
$\mathrm{~A} 5$ & $-\frac{\pi}{2}$ & 0 & $(L+P)$ & 0 \\
\hline
\end{tabular}

\begin{tabular}{ccccc} 
Link \# & $\theta_{n}$ & $d_{n}$ & $a_{n}$ & $\alpha_{n}$ \\
\hline $\mathrm{A} 7$ & $\psi$ & 0 & 0 & $\pi$ \\
$\mathrm{A} 8$ & 0 & $d_{s}$ & 0 & $-\frac{\pi}{2}$ \\
$\mathrm{~A} 9$ & $\theta_{1 s}+\frac{\pi}{2}$ & 0 & 0 & $\frac{\pi}{2}$ \\
$\mathrm{~A} 10$ & $\theta_{1 c}-\frac{\pi}{2}$ & 0 & $P_{s y}$ & $-\frac{\pi}{2}$ \\
$\mathrm{~A} 11$ & $-\frac{\pi}{2}$ & 0 & $P_{s x}$ & 0 \\
$\mathrm{~A} 12$ & $\alpha_{9}$ & 0 & 0 & $\frac{\pi}{2}$ \\
$\mathrm{~A} 13$ & $\alpha_{10}+\frac{\pi}{2}$ & 0 & 0 & $\frac{\pi}{2}$ \\
$\mathrm{~A} 14$ & $\alpha_{11}+\frac{\pi}{2}$ & 0 & 0 & $\frac{\pi}{2}$ \\
$\mathrm{~A} 15$ & 0 & $P_{L}$ & 0 & $-\frac{\pi}{2}$ \\
$\mathrm{~A} 16$ & $\alpha_{12}-\frac{\pi}{2}$ & 0 & 0 & $\frac{\pi}{2}$ \\
$\mathrm{~A} 17$ & $\alpha_{13}+\frac{\pi}{2}$ & 0 & 0 & $\frac{\pi}{2}$ \\
$\mathrm{~A} 18$ & $\alpha_{14}$ & 0 & $P_{A}$ & 0 \\
$\mathrm{~A} 19$ & $-\frac{\pi}{2}$ & 0 & $P_{H}$ & 0 \\
$\mathrm{~A} 20$ & $\frac{\pi}{2}$ & 0 & $P_{O}$ & 0 \\
\hline $\mathrm{A} 15 \mathrm{~b}$ & 0 & $P_{L_{2}}$ & 0 & $-\frac{\pi}{2}$ \\
\hline
\end{tabular}

The DH model is split into branches: the aerodynamic branch is shown in Figure 3.7 and the control branch is shown in Figure 3.8. The aerodynamic branch transformation, from inertial point to the blade, is

$$
\mathbf{T}_{a}^{6}=\mathbf{A}_{1} \mathbf{A}_{2} \mathbf{A}_{3} \mathbf{A}_{4} \mathbf{A}_{5} \mathbf{A}_{6}^{5}
$$




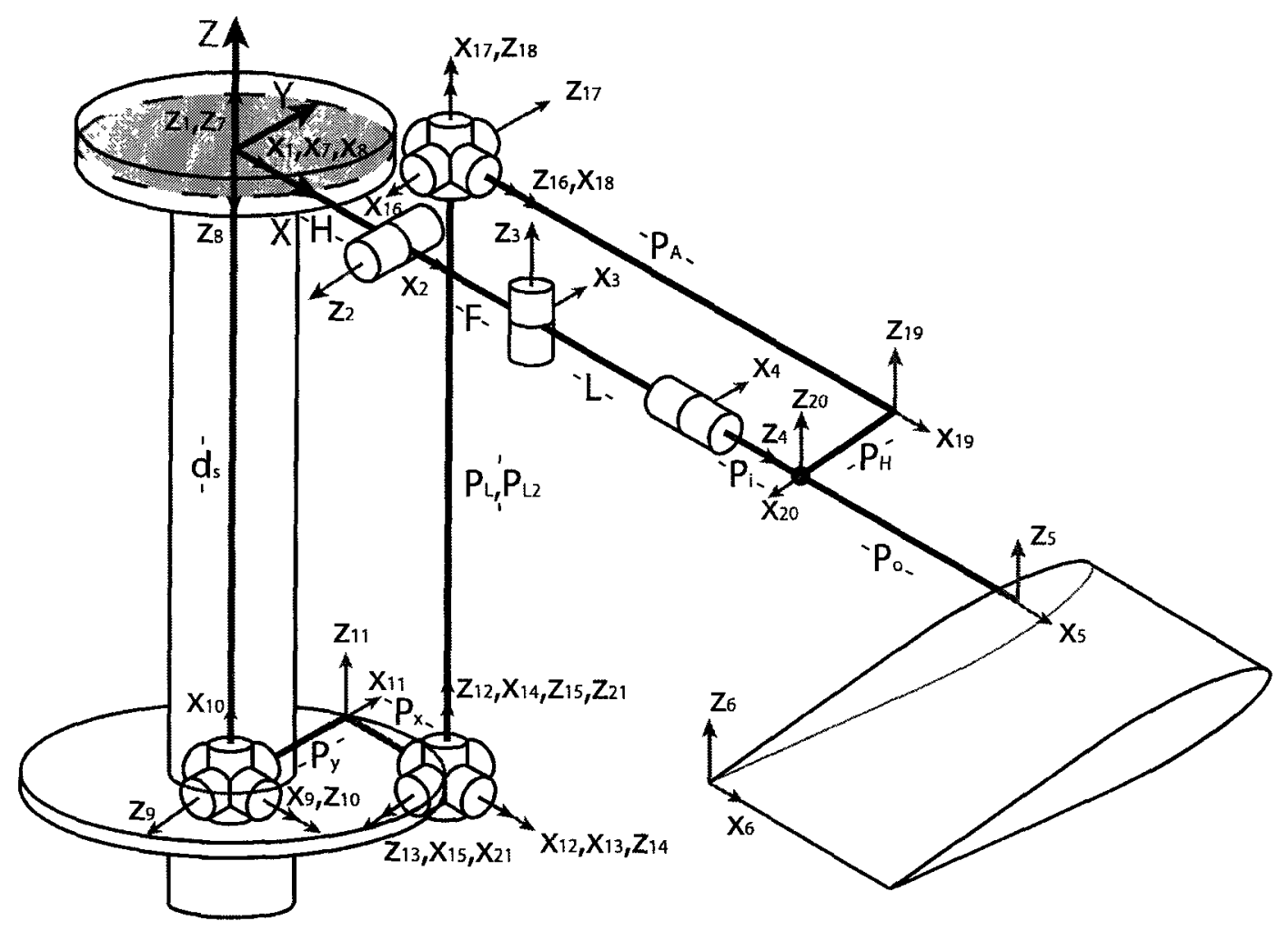

Figure 3.5: Full hub articulation with coordinate frames listed. 


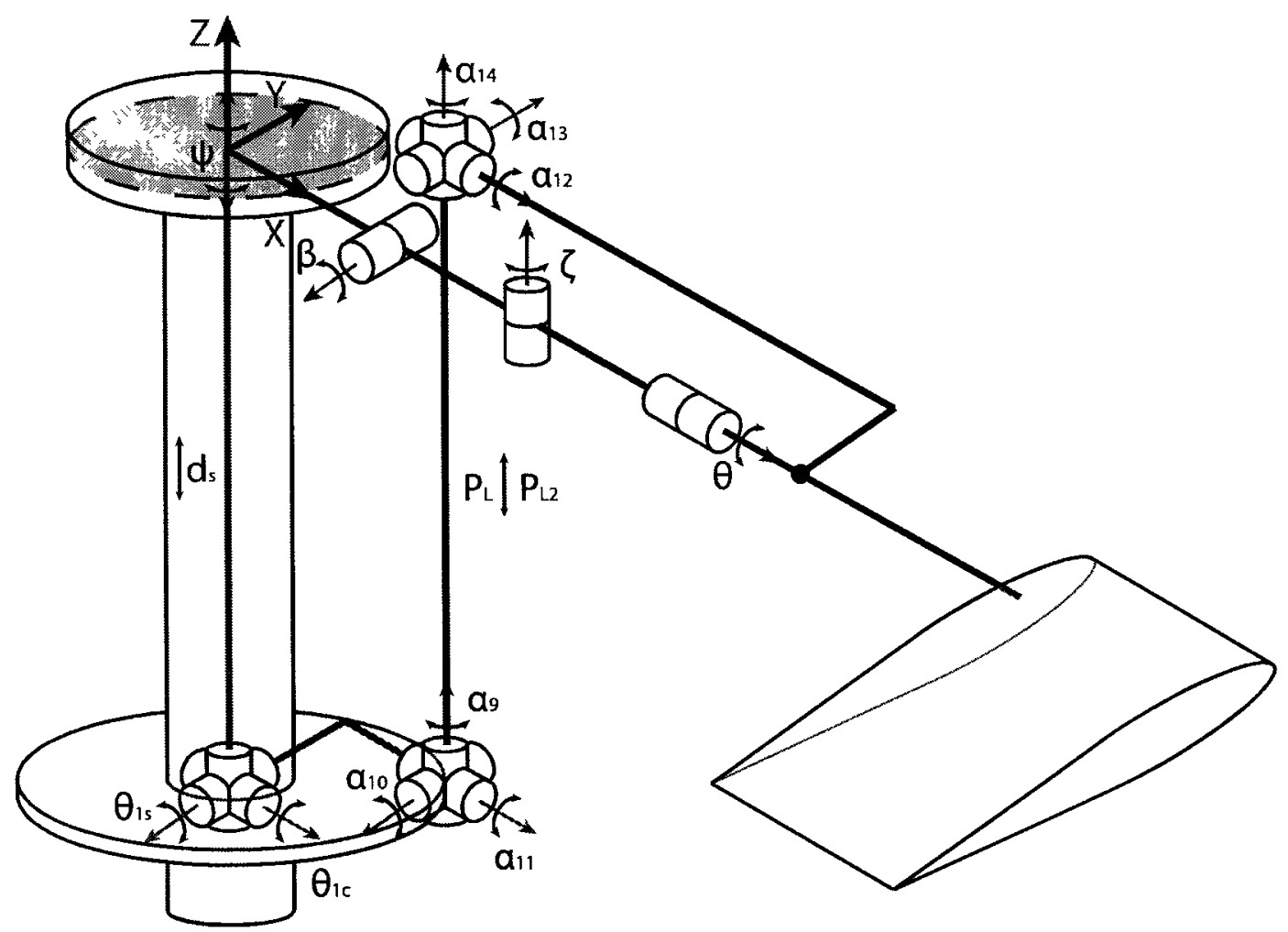

Figure 3.6: Full hub articulation with joint variables included. 


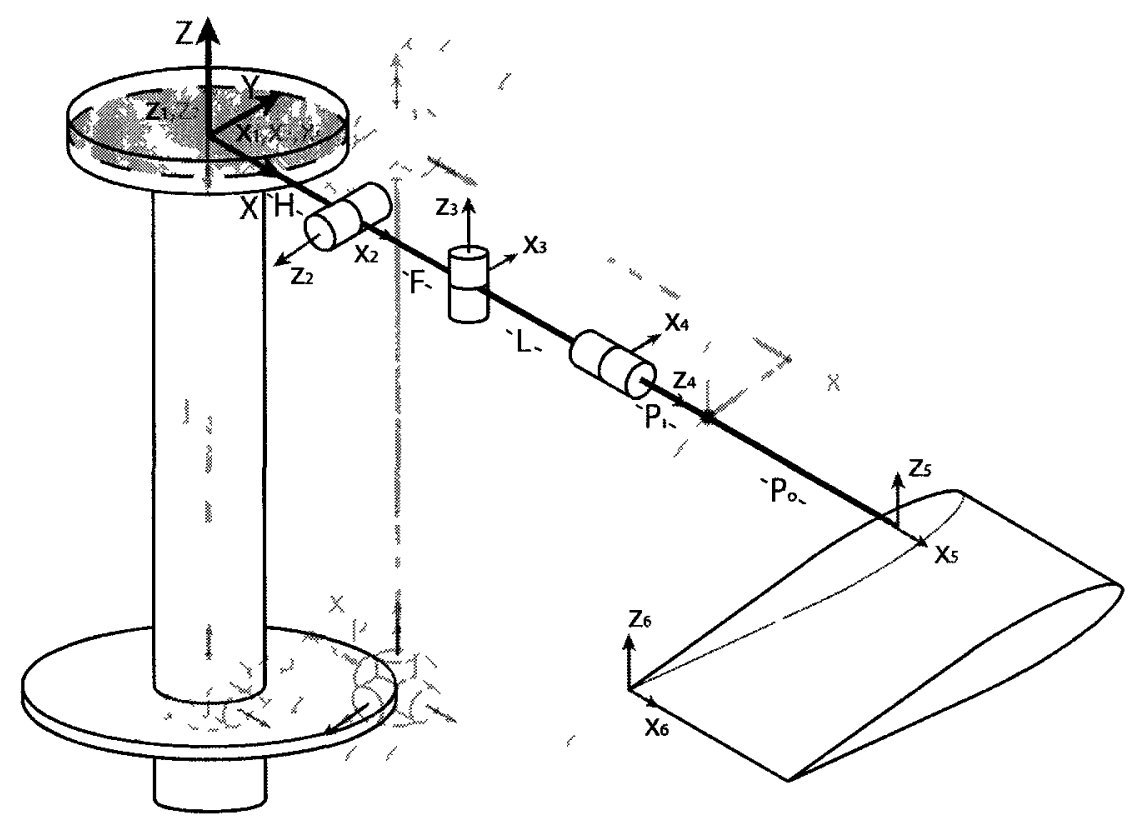

Figure 3.7: The aerodynamic chain of the helicopter hub.

with a final translation of

$$
\mathbf{A}_{6}^{5}=\left[\begin{array}{cccc}
1 & 0 & 0 & 0 \\
0 & 1 & 0 & -e c \\
0 & 0 & 1 & -\frac{t}{2} \\
0 & 0 & 0 & 1
\end{array}\right]
$$

where $e$ is the normalized distance between the trailing edge and the elastic axis, $c$ is the chord length, and $t$ is the thickness height. Clearly, this transformation does not follow the Denavit-Hartenberg formulation, but it is necessary to offset the blade root along the quarter-chord (assuming $e=\frac{3}{4}$ ) with respect to the blade coordinate system.

The control chain is given by

$$
\mathbf{T}_{c}^{6}=\mathbf{A}_{7} \mathbf{A}_{8} \cdots \mathbf{A}_{20}
$$




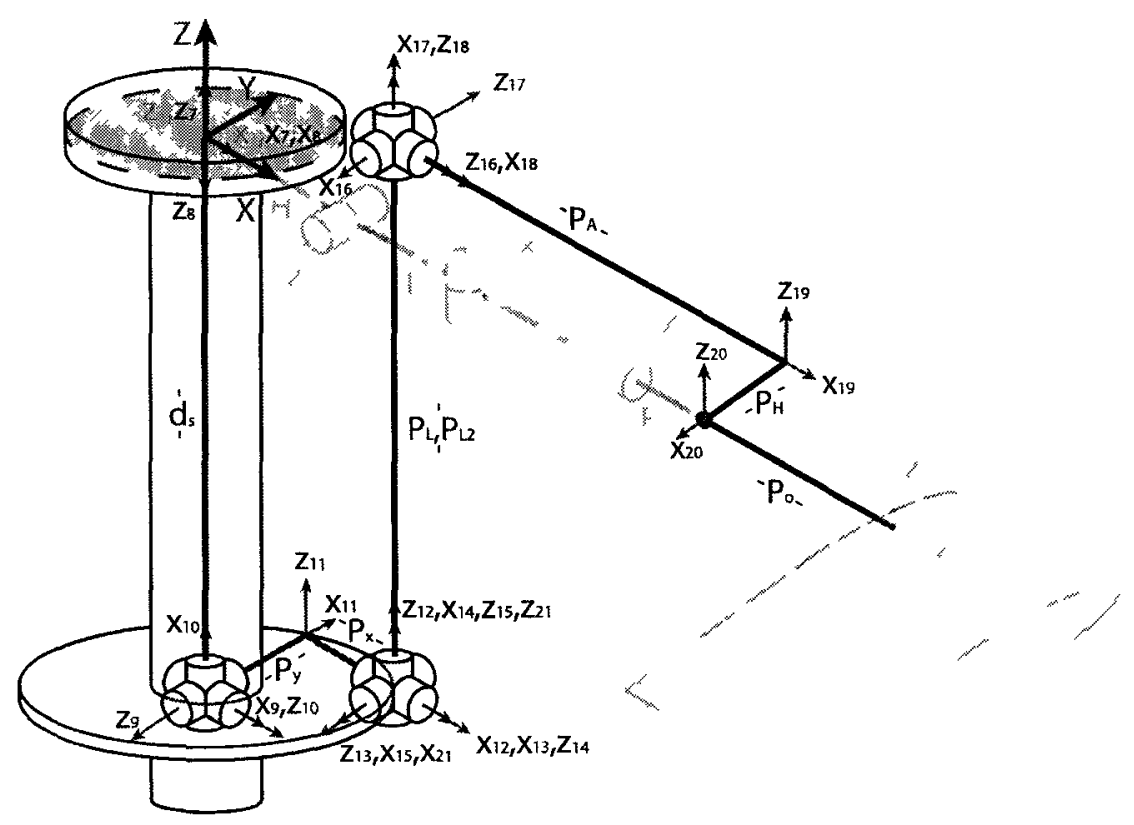

Figure 3.8: The control chain articulation of the helicopter hub.

and this branch does not end with a non-DH transformation, because it ends with the cut frame of reference on the pitch hinge link at Frame $O_{20}$. The control chain is mirrored by the third chain in the Table. This chain is not actually a chain in the system, but a means to ensure the direction of the mass of the adaptive pitch link aligns with the pitch link itself. The mass in the APL must translate only along the $z$-axis of the pitch link; therefore, it is related to that frame of reference, and the mass of the APL itself is accounted for by the transformation to $\mathbf{A}_{21}$.

Unfortunately, the equations of motion are too cumbersome to print out explicitly. Even with only 15 degrees of freedom, the matrices for the inertia and Coriolis, centripetal and gyroscopic terms are too large to be useful to show. To complete the solution, the constraints are necessary, and the entire system can then be solved using the proposed method by Arnold and Brüls, combined with GEMM, as described in the next chapter. 


\subsubsection{Rotor Articulation Holonomic Constraints}

Six constraints are necessary to close the loop after the virtual cut in the helicopter

hub articulation. Since the cut frame of reference is actually not a joint, it is a rigid spot on the chain, it is treated like a prismatic joint with an invariant $z$-axis displacement of 0 ; that is, the ends of the linkages from both chains are identically oriented and positioned. The orientation and position of the end of the link from Frame $O_{20}$ is equivalent to the end of the link in Frame $O_{5}$, and can be written using either path as

$$
\mathbf{A}_{5}^{0} \mathbf{r}_{0}=\mathbf{A}_{20}^{0} \mathbf{r}_{0}
$$

where

$$
\begin{aligned}
\mathbf{A}_{5}^{0} & =\mathbf{A}_{1} \mathbf{A}_{2} \mathbf{A}_{3} \mathbf{A}_{4} \mathbf{A}_{5} \\
\mathbf{A}_{20}^{0} & =\mathbf{T}_{c}^{6} .
\end{aligned}
$$

Equation (3.37) implies that the constraints to enforce come from

$$
\mathbf{A}_{5}^{0}=\mathbf{A}_{20}^{0}
$$

or

$$
\left[\begin{array}{cc}
\mathbf{R}_{5}^{0} & \mathbf{p}_{5}^{0} \\
\mathbf{0} & 1
\end{array}\right]=\left[\begin{array}{cc}
\mathbf{R}_{20}^{0} & \mathbf{p}_{20}^{0} \\
\mathbf{0} & 1
\end{array}\right]
$$

Therefore, the constraints are

$$
\phi_{\mathbf{d}}=\mathbf{0}=\mathrm{p}_{20}^{0}-\mathbf{p}_{5}^{0}
$$


and

$$
\phi_{\alpha \beta \gamma}=\mathbf{0}=\mathbf{R}_{5}^{0}-\mathbf{R}_{20}^{0} .
$$

The position constraints are intuitive; however, the orientation constraints require a bit more explanation, because there are nine possible equations, and only three independent orientations. The orientations at these frames of reference must not move with respect to one-another, and there are only three independent angles (such as the Roll-Yaw-Pitch angles described in the Introduction), which correspond to the three orientation constraints.

If the rotation matrices in Equation 3.43 are thought to represent Euler matrices (such as the Roll-Pitch-Yaw matrix), with only three independent angular rotations, then the difference of these matrices provide the constraint equations as differences of the independent angles. For identical orientations at the virtually cut joint, this means

$$
\alpha_{1}=\alpha_{2} \quad \beta_{1}=\beta_{2} \quad \gamma_{1}=\gamma_{2}
$$

or

$$
\Delta \alpha=0 \quad \Delta \beta=0 \quad \Delta \gamma=0,
$$

in which the subscripts 1 and 2 refer to the separate kinematic branches.

That is, if $\mathbf{R}$ is an Euler angle matrix, such as,

$$
\mathbf{R}=\left[\begin{array}{ccc}
c_{\alpha} c_{\beta} & c_{\alpha} s_{\beta} s_{\gamma}-s_{\alpha} c_{\gamma} & s_{\alpha} s_{\gamma}+c_{\alpha} s_{\beta} c_{\gamma} \\
s_{\alpha} c_{\beta} & s_{\alpha} s_{\beta} s_{\gamma}+c_{\alpha} c_{\gamma} & s_{\alpha} s_{\beta} c_{\gamma}-c_{\alpha} s_{\gamma} \\
-s_{\beta} & c_{\beta} s_{\gamma} & c_{\beta} c_{\gamma}
\end{array}\right]
$$


for which, to be compact, the notation $c_{\alpha}=\cos \alpha$ and $s_{\alpha}=\sin \alpha$ etc, then selecting three independent indices, the same in each branch rotation matrix, will result in a set of three independent equations that yield three orientation constraints. For convenience, the chosen terms for this dissertation work are:

$$
\begin{gathered}
\cos \alpha \sin \beta=0 \\
-\sin \alpha=0 \\
\cos \alpha \sin \gamma=0
\end{gathered}
$$

Fortunately, the Euler angles themselves do not need to be solved. Equating the $R_{(2,1)}, R_{(3,1)}$, and $R_{(3,2)}$ terms in each branch rotation matrices means that those three equations can be rearranged and set equal to zero, and the three resulting equations are linearly independent equations that satisfy the orientation constraints. That is,

$$
\left.\begin{array}{l}
\mathbf{R}_{5(2,1)}^{0}-\mathbf{R}_{20(2,1)}^{0}=0 \\
\mathbf{R}_{5(3,1)}^{0}-\mathbf{R}_{20(3,1)}^{0}=0 \\
\mathbf{R}_{5(3,2)}^{0}-\mathbf{R}_{20(3,2)}^{0}=0
\end{array}\right\}=\phi_{\alpha \beta \gamma}
$$

gives the three independent equations necessary to solve the orientation constraint system. Of course, the residuals of these equations must already be near zero, otherwise root finding may tend towards other values of the three orientation angles that also result zeros.

Now, the set of equations of motion for the helicopter multibody dynamics is described by the robotic equations of motion, and the constraints are elicited by the above procedure. Again, the equations and the constraints are too long to be explicitly written in text; however, they are available with the program files run by MATLAB. 


\section{Chapter 4}

\section{Building the Aeroservoelastic Simulation}

This chapter unites aerodynamic loading theory with the elastic and rigid body theories of the previous chapters and merges them with nonlinearly controlled blade root boundary conditions. It begins with the description of aerodynamics used in the computer code and follows with the description of replacing the rigid pitch link with the APL and its control theory. Afterward, the combination of the elastic and rigid body theories begins, first with the boundary conditions at the root, then with a modified time-integration scheme.

\subsection{Aerodynamics}

The aerodynamics, as stated in the Introduction, is a simplified non-linear inflow model, but because the structural code is to be implemented with SMARTROTOR, a complex vortex method with CFD solution is not necessary. Often, Drees inflow is used with blade element momentum theory, but Mangler and Squire's inflow model matches the centre and tip airflow more accurately. Mangler and Squire's model also matches experimental results, as shown in the next chapter, better than other non-vortex methods. However, the increase in complexity to a vortex method cannot 
be justified, since the structural code will be coupled to a far superior aerodynamics package, and a balance between a method with azimuthally-variable loads and complexity struck upon the Mangler and Squire model.

\subsubsection{Momentum Theory}

The simplest approach for rotor aerodynamics is to apply momentum theory, which gives a first-order prediction of thrust, $T$, and power, $P$. In momentum theory the rotor is idealized as an infinitesimally thin actuator disc with an area of $A$, that is, there are an infinite number of blades and their thickness is zero. This theory also assumes a pressure jump of $\Delta P=\frac{T}{A}$ which is induced by a pressure discontinuity at the rotor disc plane.
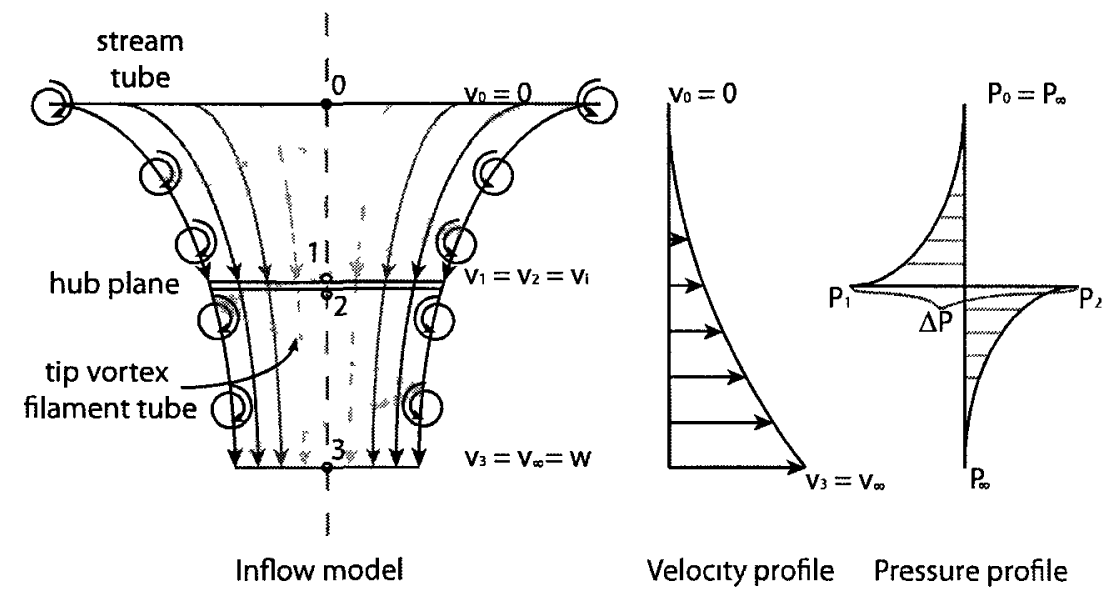

Figure 4.1: Momentum theory flow diagram. Adapted from [3].

Similar to solid mechanics, momentum theory combines the equations of Conservation of Mass, Momentum and Energy. It is assumed that the flow is one-dimensional, inviscid, incompressible, and quasi-steady. 
When the rotor is in hover, the Conservation of Mass implies

$$
\rho A_{3} w=\rho A_{2} v_{i}=\dot{m}
$$

in which the mass flow rate, $\dot{m}$ is the air flow through the rotor disc, $\rho$ is now the density of air at the helicopter altitude, and $v_{i}$ is the induced velocity of the air, due to the pressure jump of the rotor disc. The Conservation of Momentum equation in hover gives the thrust as the difference of the mass flow into the control volume and the mass flow out:

$$
\begin{aligned}
T & =\dot{m} v_{\text {out }}-\dot{m} v_{\text {in }} \\
& =\dot{m} v_{3}-0 \\
& =\dot{m} w .
\end{aligned}
$$

The Conservation of Energy can be used to determine the shaft power $P$,

$$
\begin{aligned}
-\dot{W}_{s} & =\dot{m} \frac{v_{\text {out }}^{2}}{2}-\dot{m} \frac{v_{\text {in }}^{2}}{2} \\
& =\frac{1}{2} \dot{m} w^{2} \\
& =P=T v_{i}=2 \rho A v_{i} .
\end{aligned}
$$

Normally, these results are given in non-dimensional parameters, which makes the comparison of helicopter performance simpler. In the United States these coefficients are

$$
\begin{gathered}
\lambda=\frac{v_{i}}{v_{t i p}}=\frac{v_{i}}{\Omega R} \quad c_{T}=\frac{T}{\rho A \Omega^{2} R^{2}} \\
c_{P}=\frac{P}{\rho A \Omega^{3} R^{3}} \quad c_{Q}=\frac{Q}{\rho A \Omega^{2} R^{3}} \quad Q=\frac{P}{w},
\end{gathered}
$$


where the induced velocity coefficient is $\lambda$, the torque of the rotor is $Q$, the thrust coefficient is $c_{T}$, the power coefficient is $c_{P}$, and the shaft torque coefficient is $c_{Q}$.

\section{Axial flight}

With reference to Figure 4.2 , the same analysis done above can be applied to axial climb and descent. Through the remainder of the derivation, the axial velocity $v_{c}$ is used, such that positive indicates climb and conversely, negative is descent. Normal axial climb and normal working state descent assumes that the stream-tube assumption is still valid. The same pressure profile is assumed within the control volume as in hover; therefore, this type of descent state is only valid for slow descent speeds, $0 \leq v_{d} \leq \frac{1}{2} v_{i}$
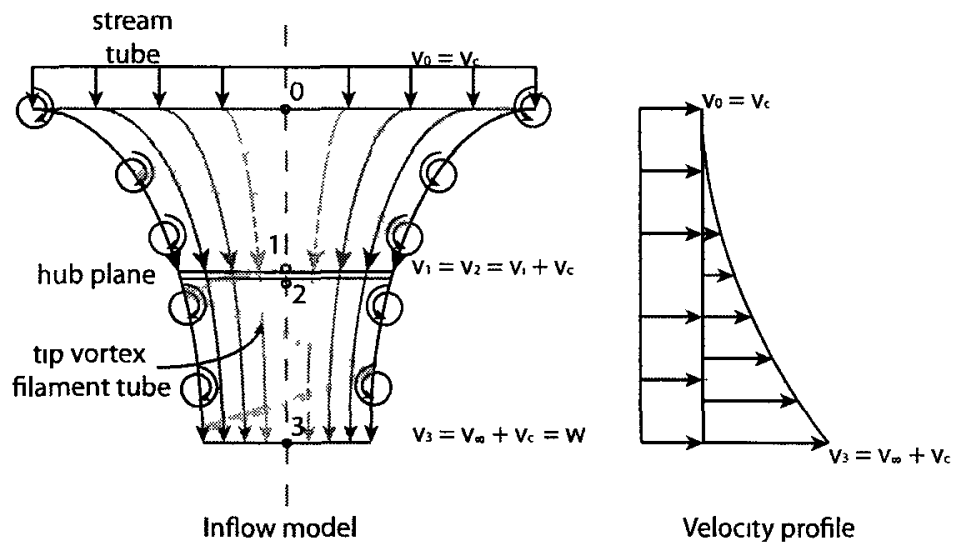

Figure 4.2: Momentum theory climb inflow diagram. Adapted from [3].

Using the momentum equilibrium, the thrust required for axial climb is

$$
T=2 \rho A\left(v_{\imath}+v_{c}\right) v_{\imath}=\dot{m} w
$$

For climb under the speed constraint above, the above equation is valid to calculate thrust. The shaft power and torque are found using this value for thrust and analysing 
it similarly to hover flight.

\section{Forward flight}

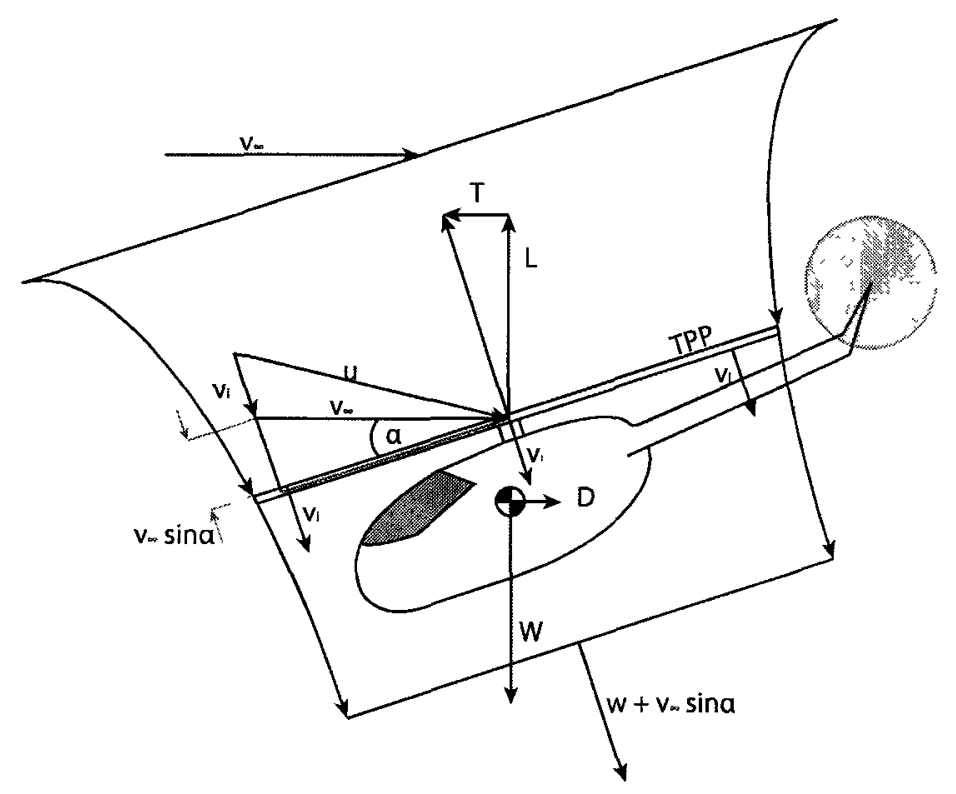

Figure 4.3: Forward flight inflow theory diagram. Adapted from [3].

In this case, as shown in Figure 4.3, the streamtube is at an angle $\alpha$ with the direction of flight. The mass flow rate depends on the resultant velocity $(u)$ through the actuator disc, such that $\dot{m}=\rho A u$, where $u=\sqrt{v_{\infty}^{2}+v_{i}^{2}}$, and the forward velocity is $v_{\infty}$. Therefore, the momentum equation yields the thrust required for forward flight, as follows:

$$
T=\dot{m}\left(w+v_{\infty} \sin \alpha\right)-\dot{m}\left(v_{\infty} \sin \alpha\right)=\dot{m} w
$$

with $\alpha$ corresponding to the rotor disc angle of attack.

The inflow velocity in forward flight also takes into account the forward velocity 
$v_{\infty}$, so normally an advance ratio $\mu$, is defined as

$$
\mu=\frac{v_{\infty} \cos \alpha}{\Omega R}
$$

The advance ratio is then used in the inflow velocity to relate the hover and forward flight inflows as

$$
\lambda=\frac{v_{\infty} \sin \alpha+v_{i}}{\Omega R}=\mu \tan \alpha+\lambda_{i}
$$

where $\lambda_{i}$ is the inflow velocity associated with hover.

\subsubsection{Blade Element Theory}

The second theory that is examined in this work is the blade element theory (BET), and it is the basis for modern analysis of rotor aerodynamics. The theory provides estimates of radial and azimuthal loading by considering each blade section as a quasitwo-dimensional airfoil producing aerodynamic loads and moments. The integration of the sectional loads over the blade span and averaging over the rotor gives the overall rotor characteristics.

At any section $y$,

$$
\left.\begin{array}{c}
u_{p}=v_{c}+v_{i} \\
u_{t}=\Omega y
\end{array}\right\} \quad u=\sqrt{u_{p}^{2}+u_{t}^{2}}
$$

and the inflow angle $\phi$, is

$$
\phi=\arctan \left(\frac{u_{p}}{u_{t}}\right) \simeq \frac{u_{p}}{u_{t}}
$$




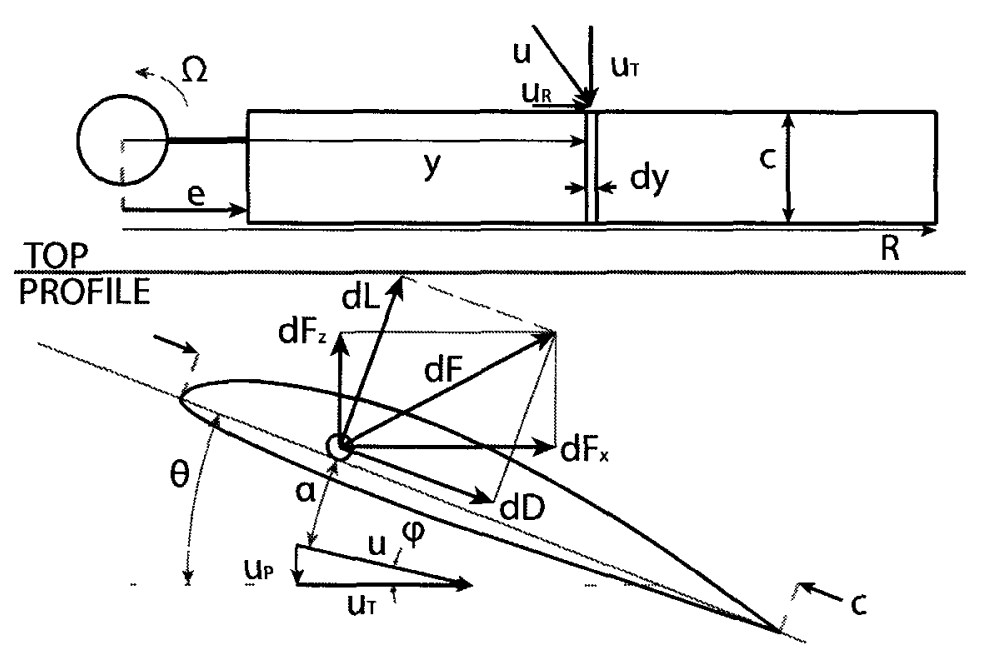

Figure 4.4: BET flow and forces diagram. Adapted from [3]

if the small angle assumption is used. The angle of attack is then given as

$$
\alpha_{\mathrm{eff}}=\theta-\phi=\theta-\frac{u_{p}}{u_{t}}
$$

where $\theta$ is the local pitch of the blade at location $y$. The infinitesimal forces and moment are

$$
\begin{aligned}
d L & =\frac{1}{2} \rho u^{2} c_{L} c d y \\
d D & =\frac{1}{2} \rho u^{2} c_{D} c d y \\
d M & =\frac{1}{2} \rho u^{2} c_{M} c^{2} d y
\end{aligned}
$$

where $d L$ is the infinitesimal lift, $d D$ is the $\mathrm{drag}$, and $d M$ is the pitching moment about the blade axis; the $c_{L}, c_{D}$, and $c_{M}$ are the coefficient of lift, drag, and moment, respectively. Since it is desirable to obtain the thrust, torque, and power, these forces need to be transformed relative to the plane of rotation. In the plane of rotation the 
element forces are

$$
\begin{aligned}
& d F_{x}=d L \cos \phi-d D \sin \phi \\
& d F_{z}=d L \sin \phi+d D \cos \phi
\end{aligned}
$$

and the elemental thrust and torque are

$$
\begin{gathered}
d T=N_{b} d F_{z} \\
d Q=N_{b} d F_{x} y
\end{gathered}
$$

where $N_{b}$ is the number of blades on the rotor. If the assumption that the velocity perpendicular to the blade motion, $u_{p}$, is much smaller than the tangential velocity of the element, $u_{t}$, then $\phi \ll 1$, and the drag is much less than the lift. In this case, the non-dimensional coefficients are normalized and integrated to yield

$$
\begin{gathered}
c_{T}=\frac{1}{2} \sigma \int_{e}^{1} c_{L} \bar{r}^{2} d r \\
c_{Q}=\frac{1}{2} \sigma \int_{e}^{1}\left(\lambda c_{L} \bar{r}^{2}+c_{D} \bar{r}^{3}\right) d r
\end{gathered}
$$

where $\sigma$ is the solidity ratio

$$
\sigma=\frac{N_{b} c}{\pi R}
$$

which is the rotor blade area divided by the rotor disc area. The integrand is done from $e$ to 1 , because it is done on the non-dimensional rotor length $\frac{y}{R}$, and $e$ is the non-dimensional root cut-out, not to be confused with the quarter-chord. Of 
course, to evaluate these integrals, one must know $c_{L}(\bar{r}), c_{D}(\bar{r})$, and $\lambda(\bar{r})$. Using ideal assumptions, one can also show that these values are equivalent to those discovered by momentum theory.

\subsubsection{Blade Element Momentum Theory}
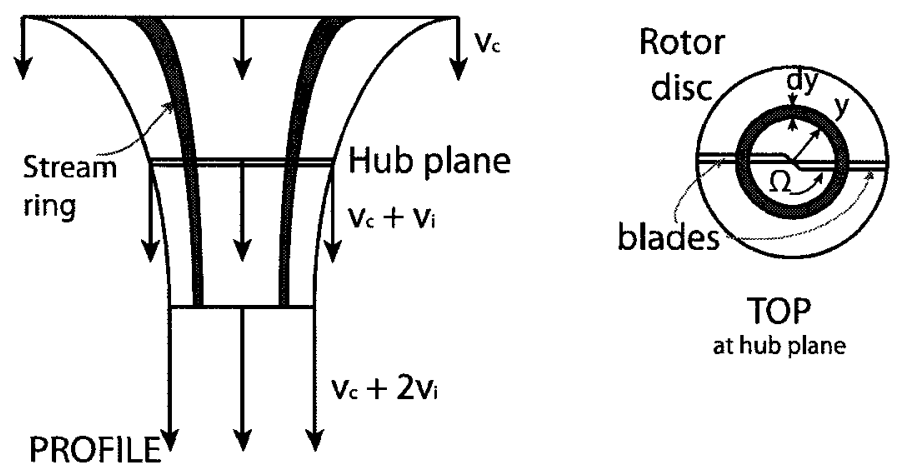

Figure 4.5: BEMT flow diagram.

Blade element momentum theory (BEMT) combines the two previous theories into a more comprehensive analysis. It uses the stream-tube analogy of the momentum theory combined with the infinitesimal segment of the blade element theory, as shown in Figure 4.5. Instead of linear elements along the blade, an annulus of the rotor disc is analysed and correlated with blade element theory.

The condition of this theory is such that neighbouring annuli have no mutual effect upon one-another. This condition is well met throughout most of the actuator disc; however, it is not accurate near the tip. Normally, for hover and axial flight, Prandtl's Circulation Loss Theory is employed to mitigate this effect, and in forward flight this effect is intrinsically matched by the Mangler and Squire pressure distributions. 
The mass flow rate over the annulus is

$$
d m=\rho\left(v_{c}+v_{i}\right) d A=2 \pi \rho\left(v_{c}+v_{i}\right) y d y
$$

so the incremental thrust, which includes climb or descent, is again the difference of the mass flows in and out of the control volume. Therefore, using BEMT, the thrust is

$$
\begin{gathered}
d T=d \dot{m} v_{o u t}-d \dot{m} v_{i n} \\
=d \dot{m}\left(v_{c}+2 v_{i}-v_{c}\right) \\
=2 v_{i} d \dot{m} \\
=4 \pi \rho\left(v_{c}+v_{i}\right) v_{i} y d y
\end{gathered}
$$

which, changed to the incremental non-dimensional thrust coefficient, is

$$
d c_{T}=\frac{d T}{\rho A \Omega^{2} R^{2}}=\frac{4 \pi \rho\left(v_{c}+v_{i}\right) v_{i} y d y}{\rho \pi R^{2} \Omega^{2} R^{2}}
$$

so that the infinitesimal coefficient of thrust is

$$
d c_{T}=4 \lambda \lambda_{i} r d r=4 \lambda\left(\lambda-\lambda_{c}\right) r d r
$$

Here $\lambda$ is still the non-dimensional inflow velocity, and $\lambda_{c}$ is the non-dimensional climbing inflow velocity, which is zero in hover. This equation for the thrust coefficient is valid for any $\lambda(r)$.

From BET, the infinitesimal thrust coefficient for axial flight, Equation (4.14) can be evaluated and becomes

$$
d c_{T}=\frac{1}{2} \sigma c_{L_{\alpha}}\left(\theta r^{2}-\lambda r\right) d r
$$


where $c_{L_{\alpha}}$ is the lift-slope curve.

Now Equations (4.18) and (4.19) can be equated to yield the inflow velocity as a function of the non-dimensional radial position $r$. Setting the thrust coefficients equal produces

$$
\begin{aligned}
4 \lambda\left(\lambda-\lambda_{c}\right) r d r & =\frac{1}{2} \sigma c_{L_{\alpha}}\left(\theta r^{2}-\lambda r\right) d r \\
\lambda\left(r, \lambda_{c}\right) & =\left(\left(\frac{\sigma c_{L_{\alpha}}}{16}-\frac{\lambda_{c}}{2}\right)^{2}+\frac{\sigma c_{L_{\alpha}}}{8} \theta r\right)^{\frac{1}{2}}-\left(\frac{\lambda_{c}}{2}+\frac{\sigma c_{L_{\alpha}}}{16}\right) .
\end{aligned}
$$

Finally, the thrust coefficient can be evaluated as before, remembering that some of these terms depend only on the local position along the blade, such as pitch, $\theta(r)$; the airfoil lift-curve, $c_{L_{\alpha}}(r)$; and blade solidity, $\sigma(c(r))$. So, the thrust for axial flight has been determined, but accurate forward flight thrust requires an additional complexity: inflow variation along the azimuth angle. In axial flight, it is clear that the inflow velocity is constant along any azimuth angle $\psi$, assuming steady-state conditions, but for forward flight that is no longer true. There are theories that include linear coefficients for forward flight, which are functions of the advance ratio and skew angle, etc. However, a model proposed by Mangler and Squire [85] produces very realistic results without including the complexity of tip vortices.

\section{The Development of Nonlinear Inflow Velocity}

Forward flight in BEMT is non-trivial because of the plunging motion from flapping. Also, as mentioned, there is periodic variation of the local velocity, which complicates the inflow through the actuator disc: ignoring any fluid-structure interactions, there can be transonic and reversed flow regions (Figure 1.2), and a skewed rotor wake. As such, it is necessary to obtain the inflow velocity as a function of both the radial position and the azimuthal position. 
Mangler and Squire treated the rotor as a lifting surface with a pressure discontinuity across the hub plane. Their reasoning is that the thrust of the rotorcraft comes from the pressure jump between the two sides of the disc. They used a rotor loading distribution similar to a typical rotor and obtained an exact solution for the induced velocity for any point on that rotor model. The two main assumptions in their model include considering an infinite number of blades, so that the rotor can be replaced by a circular disc with a pressure jump between faces; and that the disc is only lightly loaded so that the induced velocities are small compared to the stream velocity [85], which means their model is not suitable for hover.

They linearized Euler equations of motion for an elemental volume of fluid to obtain

$$
-V \frac{\partial \mathbf{v}}{\partial x}=-\frac{1}{\rho} \nabla p
$$

where $\mathbf{v}$ is the velocity field, $V$ is the volume, $\rho$ is the density of the atmosphere, and $p$ is the static pressure. The continuity equation must also be satisfied, and so from

$$
\nabla \cdot \mathbf{v}=0
$$

the result is Laplace's equation:

$$
\nabla \cdot \nabla p=\nabla^{2} p=0
$$

The solution of Laplace's equation involves choosing functions continuous everywhere except the discontinuity at the disc. Mangler and Squire determined two types of loadings, called type I and type III, that were axisymmetric to satisfy the equation, and discovered that a linear combination of these two types lead to a reasonable 
approximation of forward flight loads.

Their results are represented by a Fourier series yielding the inflow over the hub:

$$
\lambda_{i}(r, \psi)=\left(\frac{2 c_{T}}{\mu}\right)^{\frac{1}{2}}\left(\frac{c_{0}}{2}+\sum_{n=1}^{\infty}(-1)^{n} c_{n}(r, \alpha) \cos n \psi\right)
$$

where for type I loading the coefficients are

$$
\begin{aligned}
& c_{0}=\frac{3}{4} \nu \\
& c_{1}=-\frac{3 \pi}{16}\left(1-\nu^{2}\right)^{\frac{1}{2}}\left(\frac{1-\sin \alpha}{1+\sin \alpha}\right)^{\frac{1}{2}},
\end{aligned}
$$

with $\nu^{2}=1-r^{2}$, and for $c_{n}$ where $n$ is even,

$$
c_{n}=(-1)^{\frac{n-2}{2}}\left(\frac{3}{4}\right)\left(\frac{\nu+n}{n^{2}-1}\right)\left(\frac{1-\nu}{1+\nu}\right)^{\frac{n}{2}}\left(\frac{1-\sin \alpha}{1+\sin \alpha}\right)^{\frac{n}{2}}
$$

and $c_{n}=0$ for the remaining odd $n$. For type III (so called because of the distribution of the load) the coefficients are

$$
\begin{aligned}
& c_{0}=\frac{15}{8} \nu\left(1-\nu^{2}\right) \\
& c_{1}=\frac{15 \pi}{256}\left(5-9 \nu^{2}\right)\left(1-\nu^{2}\right)^{\frac{1}{2}}\left(\frac{1-\sin \alpha}{1+\sin \alpha}\right)^{\frac{1}{2}} \\
& c_{3}=\frac{45 \pi}{256}\left(1-\nu^{2}\right)^{\frac{3}{2}}\left(\frac{1-\sin \alpha}{1+\sin \alpha}\right)^{\frac{3}{2}}
\end{aligned}
$$

and again, for even $n$,

$$
c_{n}=(-1)^{\frac{n-2}{2}} \frac{15}{8}\left[\left(\frac{n+\nu}{n^{2}-1}\right)\left(\frac{9 \nu^{2}+n^{2}-6}{n^{2}-9}\right)+\frac{3 \nu}{n^{2}-9}\right]\left(\frac{1-\nu}{1+\nu}\right)^{\frac{n}{2}}\left(\frac{1-\sin \alpha}{1+\sin \alpha}\right)^{\frac{n}{2}}
$$

with $c_{n}=0$ for the odd $n$. 
Finally, the pressure field is weighted across the disc, and Leishman [3], among others, suggest an equal weighting of both type I and type III loads. Equal weights for both distributions give good longitudinal results and better flow representation near the edges of the rotor disc. From these load distributions, the thrust and drag can be calculated with BEMT and used as non-uniform loads to examine the vibration suppression characteristics of the APL in forward flight.

\subsection{Control Theory and Application}

The objective of this thesis is to show the reduction of the vibration on the rotor blade, which is akin to demonstrating the ability to control one elastic excited mass. The control algorithm uses the relative velocity and coordinate frame velocity of the pitch link to determine the proper state, on or off, of the APL. Using the momentum of the masses, and the friction forces that remove heat energy and oppose the undesirable motion, the APL slows the motion of the rotor blade. Therefore, the controller is based on generalized velocities, which produces nonlinear force boundary conditions.

\subsubsection{Overview of the Adaptive Pitch Link}

The adaptive pitch link is one of the three mechanisms suggested to dampen the vibration and noise in the SHARCS project, and it uses actively variable impedance to vary the stiffness, mass, and damping characteristics of the articulated rotor system. To show the effectiveness of the APL, it has been inverted in the simulations, since the rotor frame is not modelled. This will show the control efficacy on the rotor blades, something already described by the aeroservoelastic code.

To control the blade motion, the APL replaces the rigid pitch link, but the remaining links and hinges are unaffected. The APL system consists of two springs, 


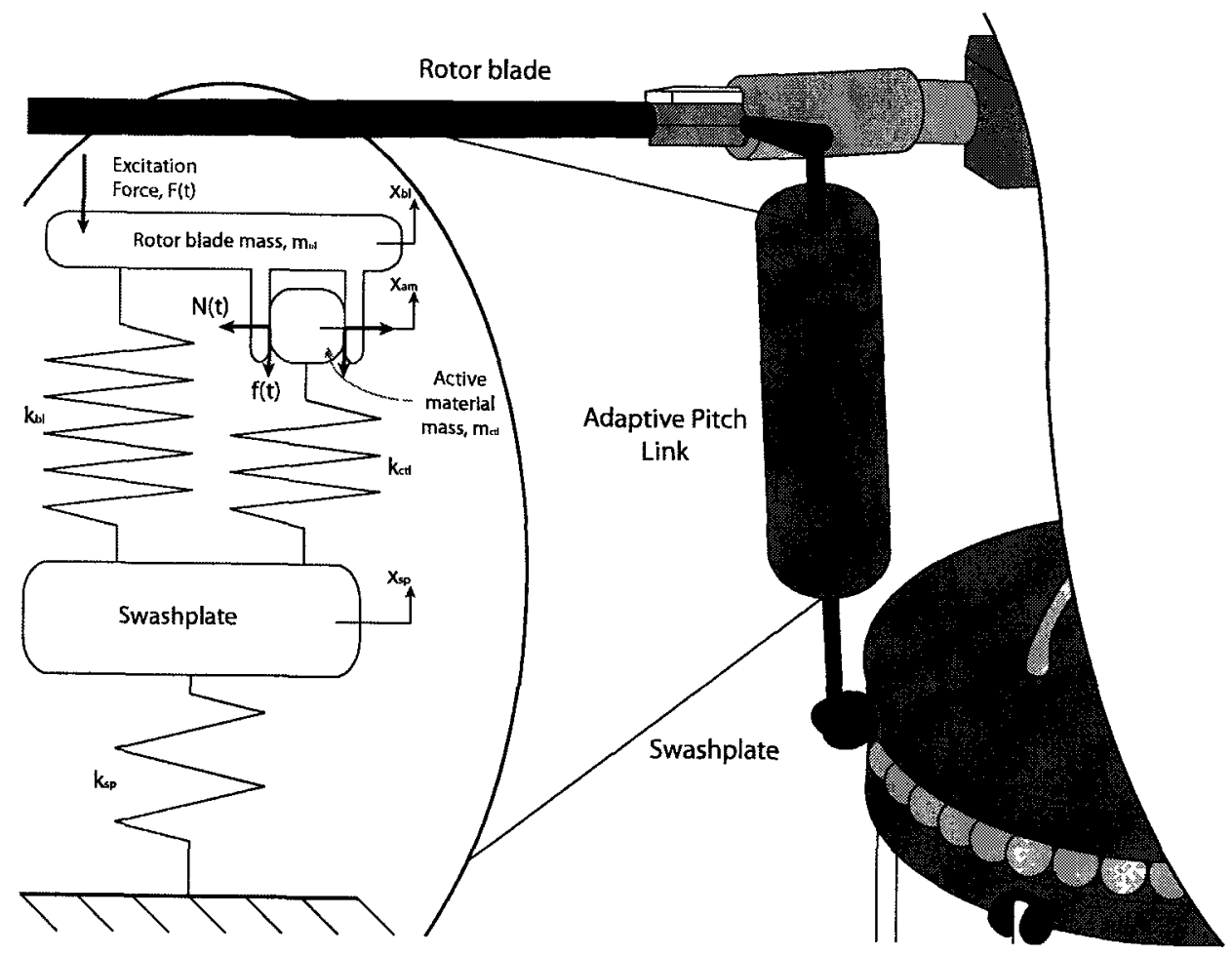

Figure 4.6: Adaptive pitch link attached to swashplate and blade root.

an actuated mass, the controlled mass, and a third mass that corresponds to the swashplate. This arrangement is shown schematically in Figure 4.6. The degree of freedom corresponding to the extension of the pitch link $\mathbf{q}_{P L}$, is

$$
\mathbf{q}_{P L}=\mathbf{x}_{b l}-\mathbf{x}_{s p}
$$

and the prismatic joint variable for the actuator mass $\mathbf{q}_{c t l}$, is

$$
\mathbf{q}_{c t l}=\mathbf{x}_{a m}-\mathbf{x}_{s p}
$$

The swashplate is hydraulically controlled by the pilot, so the spring/mass system represented by the swashplate is controlled directly, with its motion predetermined by 
the pilot command automatically. The rotor blade mass corresponds to the controlled mass, and the actuated mass is the mass of the actuator can of the adaptive pitch link.

The blade is attached to a stiff spring, represented by $k_{b l}$, and the actuated mass attached to a more compliant spring $k_{c t l}$. Normally, the unaltered pitch link is considered rigid, which implies extremely high, if not theoretically infinite stiffness, between the pitch arm and the swashplate. So for safety, the spring replacing the pitch link must be extremely stiff, yet compliant enough to allow controllable motion between its two end points.

The adaptive pitch link operates with a piezoceramic stack to elongate a mass perpendicular to the direction of motion of the link, causing friction. The excitation force, the aerodynamic forces applied to the elastic blade, corresponds to $F(t)$, which applies along the blade quarter-chord that is not shown in the figure. The mass, when stretched, contacts the slot walls on the controlled mass, which causes frictional forces to dynamically couple the three masses. The control force is a normal force, $N(t)$, acting upon the walls of the sleeve. The friction forces act in equal and opposite directions, opposing the relative motion of the masses, and have a magnitude calculated by the product of the friction coefficient $\nu$, and the normal force, as $f(t)=\nu N(t)$. Therefore, the friction force controls the vibratory motion of the blade, or alternatively they oppose the forces from the blade to the swashplate.

\subsubsection{Controlling Boundary Conditions}

The control algorithm as presented in Harold's thesis [8] is essentially unchanged. The controller switches on when the momentum difference would decrease the motion of the rotor blade. The controller switches off when the motion of the actuated mass amplifies the motion of the blade. This type of control is semi-active and indirect, 
because the work performed by the normal forces produced by applying a voltage to the actuator is independent of the displacements of the masses and the excitation forces. Hence, no direct transfer function can describe the relationship between the control forces and the modal displacement outputs, which makes this control difficult to examine in the frequency domain.

The adaptive pitch link achieves control in an elastic body by changing the boundary conditions of the body, which alters its strain energy distribution. By changing the strain energy function of the elastic body, the body does work against the excitation forces. In this case, the boundary conditions are stiffened and softened as necessary, so the rotor blade does work against the aerodynamic forces causing the vibration.

The increased frictional coupling between the two masses occurs and the aerodynamic load originally carried by the pitch link is divided between the main APL spring and the actuator spring. Additionally, damping in the form of friction heating from the sliding motion between the masses occurs. This damping is present when there is contact at the friction surface and a non-zero relative velocity between the masses. If the friction forces couple the system, the relative velocity will become zero and the frictional forces would reach a maximum as the coefficient of friction increases toward its static value, but the damping then drops to zero, too.

The equations of motion of the actuator pitch link system can be determined similarly to the open chains of the previous chapter, using the link Lagrangian and Lagrange's Equation. Considering only the degrees of freedom of the prismatic joint attached between the swashplate to the pitch horn, and the secondary prismatic joint 
representing the active controller mass, the kinetic and potential energies are

$$
\begin{aligned}
T & =\frac{1}{2} m_{b l} \dot{q}_{P L}^{2}+\frac{1}{2} m_{c t l} \dot{q}_{c t l}^{2}+\frac{1}{2} I_{P L} \omega_{P L}^{2} \\
V & =\frac{1}{2} k_{b l} \dot{q}_{P L}^{2}+\frac{1}{2} k_{c t l} \dot{q}_{c t l}^{2}+m_{b l} g h_{P L}+m_{c t l} g h_{c t l} .
\end{aligned}
$$

where the $\dot{q}$ terms represent the motion of the hinges themselves and $\omega$ is the rotation of the link fixed to the joint. Since the actuator mass is modelled as a point, there is no mass moment of inertia $(I)$ in its local frame of reference. Fortunately, the velocities and the potential energies of the prismatic joints were computed in the Multibody section, where the Lagrangian was previously encountered. However, the generalized system forces $\mathbf{Q}_{\mathbf{q}}$, are not always zero in Lagrange's Equation:

$$
\frac{d}{d t} \frac{\partial L}{\partial \dot{\mathbf{q}}}-\frac{\partial L}{\partial \mathbf{q}}=\mathbf{Q}_{\mathbf{q}}
$$

The generalized forces are lumped together in that term; however, they can be considered the combination of the damping due to heat generation and the coupling friction force. The damping due to the friction is mathematically included by adding a time-variable damping ratio into the summation for the generalized forces. The generalized forces, in that case, are

$$
\begin{aligned}
Q_{P L} & =-c \dot{q}_{P L}+f_{f r i c} \\
Q_{c t l} & =-c \dot{q}_{c t l}-f_{f r i c}
\end{aligned}
$$

Now, the joint torques obtained from the previous chapter, in Equation (3.24), can 
be equated to the generalized forces such that

$$
\mathbf{M}(\mathbf{q}) \ddot{\mathbf{q}}=\mathbf{f}(\mathbf{q}, \dot{\mathbf{q}}, t)+\mathbf{Q}_{\mathbf{q}}-\mathbf{G}_{\mathbf{q}}^{\top} \boldsymbol{\lambda}
$$

or

$$
\mathbf{M}(\mathbf{q}) \ddot{\mathbf{q}}+\mathbf{C}(\mathbf{q}, \dot{\mathbf{q}}, t) \dot{\mathbf{q}}-\mathbf{f}_{e x t}+\mathbf{K q}+\mathbf{G}_{\mathbf{q}}^{\top} \boldsymbol{\lambda}=0
$$

where

$$
\begin{gathered}
\mathbf{M}(\mathbf{q})=\mathbf{D}_{i j} \\
\mathbf{C}(\mathbf{q}, \dot{\mathbf{q}}, t) \stackrel{\bullet}{=} \mathbf{D}_{i j k} \cdot \dot{\mathbf{q}}+\mathbf{Q}_{\mathbf{q}}
\end{gathered}
$$

such that the elements of $\mathbf{Q}_{\mathbf{q}}$ have the damping coefficients of the individual joints. The friction forces are summed with the external forces, and the derivative of the constraint vector is

$$
\mathbf{G}_{\mathbf{q}}=\frac{\partial \phi_{\alpha \beta \gamma}}{\partial \mathbf{q}}
$$

which is multiplied by the Lagrange multiplier force constraints, $\boldsymbol{\lambda}$.

\section{Control Algorithm}

The control rules are simple in that they involve a state-switching between off and on, depending on the relative velocity of the masses and the absolute velocity of the controlled mass. Because the APL is axisymmetric, the velocities of the pitch link mass and actuator mass must be in the same direction, which means the relative 
velocity is the difference in speed between the two objects. Therefore, the relative velocity is

$$
v_{r e l}=\left(\dot{q}_{P L}-\dot{q}_{c t l}\right)
$$

When the relative speed is non-zero, the friction forces can be calculated by the relationship:

$$
f(t)=\left\{\begin{array}{cc}
\nu_{k} N(t) & \text { for }\left(v_{r e l}>0\right) \\
-\nu_{k} N(t) & \text { for }\left(v_{r e l}<0\right) \\
\nu_{s} N(t) & \text { for }\left(v_{r e l}=0\right)
\end{array}\right.
$$

where $\nu_{k}$ is the kinetic coefficient of friction. The kinetic coefficient of friction is nonlinearly dependent on sliding speeds, so a table in the next subsection gives the sliding steel-on-steel contact values. If the relative motion between the pitch link and the actuator stops, that is, the actuator is frictionally locked to the pitch link, then the coefficient of static friction is used.

Equation (4.37) is discretized in time using the Generalized- $\alpha$ method, in the same manner as Equation (4.33). It is included as part of the external force vector, and is manipulated in the same manner. In the computer code, the previous time step velocities are used, which provides better stability of the control forces. This change minorly affects the control of the system, as long as the time step is small relative to the motion of the system. Control sliding, a nonlinear physical effect on controlled systems, was not analysed in this thesis.

Assuming the control can be in only one of the two states, then the primary concern of the control algorithm is to determine which state is appropriate. The control depends on the relative speeds of the pitch link and the actuated mass, but the motion of the actuator could increase the vibration of the blade if the momentum 
transfer enhances its motion. Therefore, the decision to switch state is based on the relative velocity of the pitch link and actuator and also the absolute velocity of the pitch link itself.

Considering the two velocity terms, the relative and absolute velocities, separately, shows that there are four cases to determine whether to switch on or off:

- Case 1: $\dot{q}_{P L}>0$ and $\dot{q}_{P L}-\dot{q}_{c t l}>0$;

- Case 2: $\dot{q}_{P L}>0$ and $\dot{q}_{P L}-\dot{q}_{c t l}<0$;

- Case 3: $\dot{q}_{P L}<0$ and $\dot{q}_{P L}-\dot{q}_{c t l}>0$;

- Case 4: $\dot{q}_{P L}<0$ and $\dot{q}_{P L}-\dot{q}_{c t l}<0$.

In Case 1, the pitch link moves in the positive direction, and the relative speed between the link and actuator is also positive. In this case, the friction forces promote motion of the controlled structure, so the control should be switched off. Case 2 shows that the controlled mass is moving in the positive sense, but the relative velocity is negative. Therefore, the friction forces will oppose the motion of the pitch link, so the controller should be switched on. Similar to the previous case, Case 3 indicates that the motion of the pitch link and the relative motion are in opposite directions. Again, the controller should be switched on for this case, as the resulting friction forces will dampen the motion of the blade. Case 4 is similar to the first case, in that the speeds have the same sense, this time both negative. For Case 4, the controller should be switched off to prevent acceleration of the blade.

These four cases can be summarized by a simple relationship:

$$
u=\dot{q}_{P L}\left(\dot{q}_{c t l}-\dot{q}_{P L}\right)
$$


When $u>0$, the controller should be off, when $u<0$, the controller should be switched on. If $u=0$, the pitch link and the actuator have the same motion, or the pitch link is not moving, so there should be no switch in actuation. Therefore, the control algorithm is

$$
f_{\text {ctrl }}=f_{\text {fric }}=\nu N, \begin{cases}N=\hat{N}, & u<0 \\ N=0, & u>0 \\ N=N_{\text {last }}, & u=0\end{cases}
$$

\section{Sliding friction forces}

The coefficient of friction in the program comes from a look-up table from Reference [116], and is linearly interpolated from the table values by MATLAB. The table below reproduces the values in the reference.

Table 4.1: Coefficient of friction for sliding steel flat plate contacts [116].

\begin{tabular}{lccccccc}
\hline Velocity $\left[\frac{\mathrm{in}}{\mathrm{s}}\right]$ & 0.0001 & 0.001 & 0.01 & 0.1 & 1 & 10 & 100 \\
\hline Fric. Coeff. [] & 0.53 & 0.48 & 0.39 & 0.31 & 0.23 & 0.19 & 0.18 \\
\hline
\end{tabular}

The coefficient of static friction is assumed to be approximately $\nu_{s}=0.78$. Strictly speaking, there is a discontinuity between $\mu_{k}$ and $\mu_{s}$, but for simplicity, all values of $\mu$ are interpolated in MATLAB. 


\subsection{Generalized- $\alpha$ Method for Index-3 Differential Algebraic Equations}

With the completion of the constraints' description, the system can be solved in time using a similar time integration scheme as in the elastic body chapter. A modified generalized- $\alpha$ method provides the finite difference formula used in constrained multibody dynamics. The next section details the reasons for using such a method, as opposed to the alternative, which is developing a two-field structural problem, solved for position and velocity.

Considerable work has been done in the field of multibody dynamics on constrained systems, especially concerning the inclusion of differential algebraic equations (DAEs), as indicated by the brief list in the literature review. DAEs are a general form of ordinary differential equations (ODEs) involving a vector of variables and one independent variable, usually time, in which not all derivatives appear explicitly. Differential algebraic equations are common in problems with constrained motion, as the constraints themselves are typically differential functions of the mechanical variables combined with Lagrange multipliers that act as force terms.

The index of a differential algebraic equation denotes how many differentiations would be required to yield their respective ODE. For example, a DAE of index-3 requires three differentiation steps to yield a set of ordinary differential equations. (Thus, DAEs of index-0 are also ODEs.) DAEs of index-3 are quite common in constrained mechanics, yet are difficult to solve, since they involve Lagrange multipliers, which are sensitive to perturbation [117].

Because of the accuracy of the generalized- $\alpha$ method, combined with its high frequency numerical damping properties, it was chosen to solve the multibody problem posed by the helicopter hub. Arnold and Brüls [78] devised a method based on the 
generalized- $\alpha$ scheme to solve index-3 DAEs.

They start with the general system equation including constraints as

$$
\begin{aligned}
\mathbf{M}(\mathbf{q}) \ddot{\mathbf{q}} & =\mathbf{f}(\mathbf{q}, \dot{\mathbf{q}}, t)-\phi_{, \mathbf{q}}^{\top}(t) \boldsymbol{\lambda} \\
\mathbf{0} & =\phi(t)
\end{aligned}
$$

where $\mathbf{f}(\mathbf{q}, \dot{\mathbf{q}}, t)$ contains the gyroscopic, Coriolis, and centripetal matrix formulated in the Lagrangian Mechanics section, and $\phi$ is the set of constraint equations from the previous section equations. They propose that using a modified acceleration term, weighted differently than the residual equation, one can enforce the dynamic equilibrium of the system of equations. So, the time-dependent variables, $\mathbf{q}_{n+1}, \dot{\mathbf{q}}_{n+1}$, $\ddot{\mathbf{q}}_{n+1}$, and $\boldsymbol{\lambda}_{n+1}$ satisfy the residuals of Eq. (4.40), but acceleration-like variables

$$
\underbrace{\left(1-\alpha_{m}\right) \mathbf{a}_{n+1}+\alpha_{m} \mathbf{a}_{n}}_{\mathbf{a}_{n+1-\alpha_{m}}}=\left(1-\alpha_{f}\right) \ddot{\mathbf{q}}_{n+1}+\alpha_{f} \ddot{\mathbf{q}}_{n}
$$

are introduced, with $\mathbf{a}_{0}=\ddot{\mathbf{q}}_{0}$, to yield a modified-Newmark scheme.

Recall the generalized- $\alpha$ equations from Newmark:

$$
\begin{aligned}
& \mathbf{q}_{n+1}=\mathbf{q}_{n}+\Delta t \dot{\mathbf{q}}+\Delta t^{2}\left(\frac{1}{2}-\beta\right) \mathbf{a}_{n}+\Delta t^{2} \beta \mathbf{a}_{n+1} \\
& \dot{\mathbf{q}}_{n+1}=\dot{\mathbf{q}}_{n}+\Delta t(1-\gamma) \mathbf{a}_{n}+\Delta t \gamma \mathbf{a}_{n+1}
\end{aligned}
$$

in which the $\mathbf{a}_{n+1}$ terms can be eliminated using Equation (4.42), and now the values for velocity and acceleration are relabelled with the dot-derivatives of the position, to distinguish them from the acceleration-like variables. The values of the Greekletter parameters remains the same as before; however, now the derivatives of the 
generalized- $\alpha$ velocity and acceleration are slightly different:

$$
\begin{aligned}
& \frac{\partial \ddot{\mathbf{q}}_{n+1}}{\partial \mathbf{q}_{n+1}}=\frac{1-\alpha_{m}}{\left.\Delta t^{2} \beta(1-\alpha)_{f}\right)}=\beta^{\prime} \mathbf{I} \\
& \frac{\partial \dot{\mathbf{q}}_{n+1}}{\partial \mathbf{q}_{n+1}}=\frac{\gamma}{\Delta t \beta}=\gamma^{\prime} \mathbf{I} .
\end{aligned}
$$

The dynamic tangential stiffness matrix, assuming small angles in the mass matrix, is

$$
\mathbf{K}_{T_{\mathrm{dyn}}}\left(\mathbf{q}_{n+1}^{i}\right)=\left[\begin{array}{cc}
\left(\mathbf{M} \beta^{\prime}-\mathbf{f}_{\dot{\mathbf{q}}} \gamma^{\prime}+\mathbf{K}_{T}\right) & \phi_{, \mathbf{q}}^{\mathrm{T}} \\
\phi,,_{\mathbf{q}} & \mathbf{0}
\end{array}\right]
$$

with the tangential stiffness matrix given as

$$
\mathbf{K}_{T}=\frac{\partial\left(\mathbf{M}(\mathbf{q}) \ddot{\mathbf{q}}-\mathbf{f}(\mathbf{q}, \dot{\mathbf{q}}, t)+\phi,_{\mathbf{q}}^{\top}(t) \boldsymbol{\lambda}\right)}{\partial \mathbf{q}}
$$

Rigid body mechanics are notoriously ill-posed problems, so Bottasso et al. [118] proposed a scaling technique that is applied here. After linearization, the residual equation is

$$
\mathbf{K}_{T_{\mathrm{dyn}}} \Delta \mathbf{y}=-\mathbf{r}
$$

where $\mathbf{r}$ is the residual of Equations (4.40) and $\Delta \mathbf{y}$ is the augmented vector of joint variables $\mathbf{q}$ and Lagrange multipliers $\boldsymbol{\lambda}$, which enforce the constraints, as

$$
\Delta \mathbf{y}=\left\{\begin{array}{c}
\mathbf{q} \\
\lambda
\end{array}\right\}
$$

The scaled equation is

$$
\left(\mathbf{D}_{L} \mathbf{K}_{T_{\mathrm{dyn}}} \mathbf{D}_{R}\right) \cdot\left(\mathbf{D}_{R}^{-1} \Delta \mathbf{y}\right)=-\mathbf{D}_{L} \mathbf{r}
$$


such that

$$
\overline{\mathbf{K}}_{T_{\mathrm{dyn}}} \Delta \overline{\mathbf{y}}=-\overline{\mathbf{r}}
$$

and

$$
\begin{aligned}
& \mathbf{D}_{L}=\left[\begin{array}{cc}
\beta(\Delta t)^{2} \mathbf{I} & \mathbf{0} \\
\mathbf{0} & \mathbf{I}
\end{array}\right] \\
& \mathbf{D}_{R}=\left[\begin{array}{cc}
\mathbf{I} & \mathbf{0} \\
\mathbf{0} & \frac{\mathbf{I}}{\beta(\Delta t)^{2}}
\end{array}\right] .
\end{aligned}
$$

The procedure for solving the multibody dynamics is similar to that of the continuum mechanics, so that will not be reviewed now.

\subsection{Elastodynamic Blade with Articulated Hub Boundary Conditions}

This section is split into two parts: the coupling of the elastic boundary conditions with the rigid body hub articulation, and the solution of the constrained system. The boundary conditions on the root arise from the dependency of the aerodynamics on the position of the blade, which in turn are affected by the aerodynamics. Instead of an iterative solver, the simultaneous solution of the aerodynamic chain hinges and the position of the blade root can be found by assuming another set of Lagrange multipliers that restrict the motion. 


\subsubsection{Coupling Elastic Boundary Conditions with Hub Ar- ticulation}

The orientation of the blade must be known for the surface forces, the aerodynamics, to act properly. The orientation of the blade comes from the position and orientation of the blade root, which can be given in terms of the aerodynamic chain. In fact, if one knows the position of the blade root for multiple points on the blade root surface, then the orientation is known also. Therefore, the position of the blade root surface is the only constraint necessary to couple the elastic body motion of the blade with the rigid body articulation of the chain.

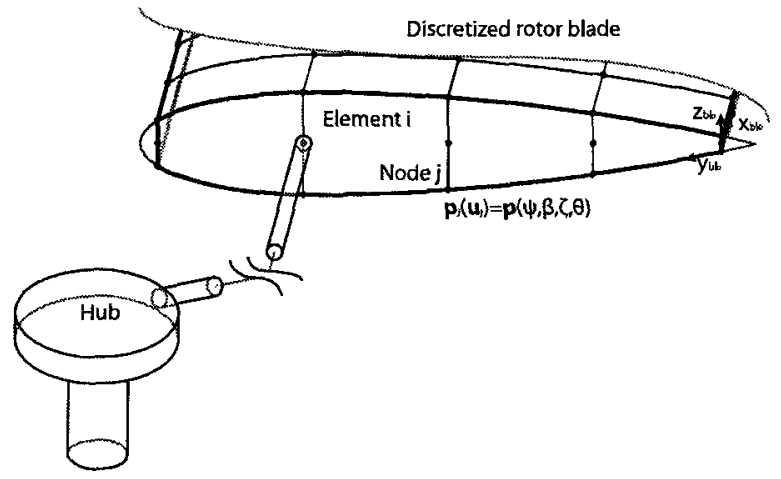

Figure 4.7: Finite element discretized elements constrained by aerodynamic chain.

To begin, it is assumed that the constraints can be satisfied on the elastic element nodes, which implies a pointwise constraints. This will make the Lagrange multipliers sensitive to perturbation, since the restriction on the element nodes conflicts with the Poisson ratio contraction at the body surfaces. In fact, the assumption that the blade root is rigid compared to the remainder of the blade is acceptable: the blade root is extremely stiff compared to the remainder of the blade. The blade root attachment is often clamped to the articulation, and in composite blades, the root typically has many more plies of laminae than the aerodynamically efficient sections of the blade. 
Since the position of the blade root nodes must be equal to the position of the end effector from the articulation, as shown in Figure 4.7,

$$
\mathbf{p}_{i}\left(\mathbf{u}_{i}\right)=\mathbf{p}_{i}(\mathbf{q})
$$

In this Equation, the position of the root is a function of the elemental nodes, when considering it as part of the blade, and it is a function of the hinge positions when considering it as the end effector from the articulation. In this case,

$$
\mathbf{p}_{i}(\mathbf{q})=\mathbf{p}_{i}(\psi, \beta, \zeta, \theta)
$$

Therefore, the constraint equation is

$$
\phi_{\mathbf{u}}=\mathbf{p}_{i}\left(\mathbf{u}_{i}\right)-\mathbf{p}(\mathbf{q})=\mathbf{0}
$$

and again, writing the constraints in full for a generic set of hinges is inconvenient.

\subsubsection{Coupling Elastic and Rigid Body Equations}

The full equations of motion with control forces and damping added and the constraints on both the closed chain and the blade root are

$$
\begin{aligned}
\mathbf{M}_{\mathbf{q}} \ddot{\mathbf{q}}+\mathbf{C}_{\mathbf{q}} \dot{\mathbf{q}}+\mathbf{K q}-\mathbf{f}_{e x t ; \mathbf{q}}+\mathbf{G}_{\mathbf{q}}^{\top} \boldsymbol{\mu} & =\mathbf{0} \\
\phi_{\mathbf{q}} & =\mathbf{0} \\
\mathbf{M}_{\mathbf{d}} \ddot{\mathbf{d}}+\mathbf{f}_{i n t}-\mathbf{f}_{e x t ; \mathbf{d}}+\mathbf{G}_{\mathbf{d}}^{\top} \boldsymbol{\lambda} & =\mathbf{0} \\
\phi_{\mathbf{d}} & =\mathbf{0}
\end{aligned}
$$


where

$$
\begin{array}{r}
\mathbf{G}_{\mathrm{q}}=\phi_{\mathrm{q}, \mathrm{q}} \\
\mathbf{G}_{\mathbf{d}}=\phi_{\mathbf{d}, \mathbf{d}},
\end{array}
$$

and

$$
\begin{array}{r}
\mathbf{f}_{e x t ; \mathbf{q}}=\mathbf{f}_{g ; \mathbf{q}}+\mathbf{f}_{c t r l} \\
\mathbf{f}_{e x t ; \mathbf{d}}=\mathbf{f}_{g ; \mathbf{d}}+\mathbf{f}_{a e r o} .
\end{array}
$$

Re-arranging Equations (4.53) and (4.55) yields

$$
\begin{aligned}
{\left[\begin{array}{cc}
\mathbf{M}_{\mathbf{q}} & \mathbf{0} \\
\mathbf{0} & \mathbf{M}_{\mathbf{d}}
\end{array}\right]\left\{\begin{array}{c}
\ddot{\mathbf{q}} \\
\ddot{\mathbf{d}}
\end{array}\right\} } & +\left[\begin{array}{cc}
\mathbf{C}_{\mathbf{q}} & \mathbf{0} \\
\mathbf{0} & \mathbf{0}
\end{array}\right]\left\{\begin{array}{c}
\dot{\mathbf{q}} \\
\dot{\mathbf{d}}
\end{array}\right\}+\left[\begin{array}{cc}
\mathbf{K} & \mathbf{0} \\
\mathbf{0} & \mathbf{0}
\end{array}\right]\left\{\begin{array}{l}
\mathbf{q} \\
\mathbf{d}
\end{array}\right\}+\left\{\begin{array}{c}
\mathbf{0} \\
\mathbf{f}_{i n t}
\end{array}\right\} \\
& -\left\{\begin{array}{c}
\mathbf{f}_{e x t ; \mathbf{q}} \\
\mathbf{f}_{e x t ; d}
\end{array}\right\}+\left[\begin{array}{cc}
\mathbf{G}_{\mathbf{q}}^{\top} & \mathbf{0} \\
\mathbf{0} & \mathbf{G}_{\mathbf{d}}^{\top}
\end{array}\right]\left\{\begin{array}{l}
\mu \\
\boldsymbol{\lambda}
\end{array}\right\}=\mathbf{0}
\end{aligned}
$$

or, in matrix form, Equation (4.57) can be written as

$$
\mathbf{M}_{\mathbf{x}} \ddot{\mathbf{x}}+\mathbf{C}_{\mathbf{x}} \dot{\mathbf{x}}+\mathbf{K} \mathbf{x}+\mathbf{f}_{i n t ; \mathbf{x}}-\mathbf{f}_{e x t ; \mathbf{x}}+\mathbf{G}_{\mathbf{x}}^{\top} \boldsymbol{\kappa}=\mathbf{0}
$$

in which

$$
x=\left\{\begin{array}{c}
q \\
d
\end{array}\right\} \quad \kappa=\left\{\begin{array}{l}
\mu \\
\lambda
\end{array}\right\}
$$

and time derivatives of $\mathbf{x}$ are denoted by the dot symbols. Using the Arnold and Brüls method to describe the accelerations, Equation (4.42), in which the $\ddot{\mathbf{x}}$ replace the $\ddot{\mathbf{q}}$ 
terms, in combination with the displacement and velocity terms from the generalized$\alpha$ method, Equation (2.69), means that

$$
\begin{aligned}
\left(1-\alpha_{m}\right) \mathbf{a}_{n+1}+\alpha_{m} \mathbf{a}_{n}=-\alpha_{f} \mathbf{M}_{\mathbf{x}_{n}}^{-1}\left(\mathbf{C}_{\mathbf{x}_{n}} \dot{\mathbf{x}}_{n}+\mathbf{K} \mathbf{x}_{n}+\mathbf{f}_{i n t ; \mathbf{x}_{n}}-\mathbf{f}_{e x t ; \mathbf{x}_{n}}+\mathbf{G}_{\mathbf{x}_{n}}^{\top} \boldsymbol{\kappa}_{n}\right) \\
-\left(1-\alpha_{f}\right) \mathbf{M}_{\mathbf{x}_{n+1}}^{-\mathbf{1}}\left(\mathbf{C}_{\mathbf{x}_{n+1}} \dot{\mathbf{x}}_{n+1}+\mathbf{K} \mathbf{x}_{n+1}+\mathbf{f}_{i n t ; \mathbf{x}_{n+1}}-\mathbf{f}_{e x t ; \mathbf{x}_{n+1}}+\mathbf{G}_{\mathbf{x}_{n+1}}^{\top} \boldsymbol{\kappa}_{n+1}\right)
\end{aligned}
$$

holds for the finite-differenced time discretization. An important observation here is that there is a subtle difference Equation (4.59) and the result from Section 4.3. Instead of eliminating the $\mathbf{a}_{n+1}$ terms and solving for $\ddot{\mathbf{q}}_{n+1}$, the endpoint accelerations $\ddot{\mathbf{q}}_{n+1}$ is eliminated in favour of $\mathbf{a}_{n+1}$, which allows one to combine the GEMM approach and the Arnold and Brüls methods. This is a significant contribution to the coupling of elastic and rigid body motions.

Multiplying Equation (4.59) by the mass matrix evaluated at $t_{n+1}, \mathbf{M}_{\mathbf{x}_{n+1}}=$ $\mathbf{M}\left(\mathbf{x}\left(t_{n+1}\right)\right)$, gives

$$
\begin{array}{r}
\mathbf{M}_{\mathbf{x}_{n+1}}\left(\left(1-\alpha_{m}\right) \mathbf{a}_{n+1}+\alpha_{m} \mathbf{a}_{n}\right)=-\alpha_{f} \mathbf{I}_{T}\left(\mathbf{C}_{\mathbf{x}_{n}} \dot{\mathbf{x}}_{n}+\mathbf{K} \mathbf{x}_{n}+\mathbf{f}_{i n t ; \mathbf{x}_{n}}-\mathbf{f}_{e x t ; \mathbf{x}_{n}}+\mathbf{G}_{\mathbf{x}_{n}}^{\top} \boldsymbol{\kappa}_{n}\right) \\
-\left(1-\alpha_{f}\right)\left(\mathbf{C}_{\mathbf{x}_{n+1}} \dot{\mathbf{x}}_{n+1}+\mathbf{K} \mathbf{x}_{n+1}+\mathbf{f}_{i n t ; \mathbf{x}_{n+1}}-\mathbf{f}_{e x t ; \mathbf{x}_{n+1}}+\mathbf{G}_{\mathbf{x}_{n+1}}^{\top} \boldsymbol{\kappa}_{n+1}\right),
\end{array}
$$

if $\mathbf{M}_{\mathbf{x}_{n+1}}$ and $\mathbf{M}_{\mathbf{x}_{n}}$ are invertible. (The mass matrix would only become singular if some joints are modelled as massless.) The matrix $\mathbf{I}_{T}$ is approximately the identity matrix because

$$
\mathbf{I}_{T}=\left[\begin{array}{cc}
\mathbf{M}_{\mathbf{q}_{n+1}} & \mathbf{0} \\
\mathbf{0} & \mathbf{M}_{\mathbf{d}_{n+1}}
\end{array}\right]\left[\begin{array}{cc}
\mathbf{M}_{\mathbf{q}_{n}}^{-1} & \mathbf{0} \\
\mathbf{0} & \mathbf{M}_{\mathbf{d}_{n}}^{-1}
\end{array}\right]=\left[\begin{array}{cc}
\mathbf{M}_{\mathbf{q}_{n+1}} \mathbf{M}_{\mathbf{q}_{n}}^{-1} & \mathbf{0} \\
\mathbf{0} & \mathbf{I}
\end{array}\right]
$$


if $\Delta t$ is small. In terms of the accelerations evaluated at $t_{n+1}$,

$$
\begin{aligned}
\mathbf{M}_{\mathbf{x}_{n+1}} & \left(\left(1-\alpha_{f}\right) \ddot{\mathbf{x}}_{n+1}+\alpha_{f} \ddot{\mathbf{x}}_{n}\right)=\mathbf{M}_{\mathbf{x}_{n+1}}\left(\left(1-\alpha_{m}\right) \mathbf{a}_{n+1}+\alpha_{m} \mathbf{a}_{n}\right)= \\
& -\left(1-\alpha_{f}\right)\left(\mathbf{C}_{\mathbf{x}_{n+1}} \dot{\mathbf{x}}_{n+1}+\mathbf{K} \mathbf{x}_{n+1}+\mathbf{f}_{i n t ; \mathbf{x}_{n+1}}-\mathbf{f}_{e x t ; \mathbf{x}_{n+1}}+\mathbf{G}_{\mathbf{x}_{n+1}}^{\top} \boldsymbol{\kappa}_{n+1}\right) \\
& -\alpha_{f} \mathbf{I}_{T}\left(\mathbf{C}_{\mathbf{x}_{n}} \dot{\mathbf{x}}_{n}+\mathbf{K} \mathbf{x}_{n}+\mathbf{f}_{i n t ; \mathbf{x}_{n}}-\mathbf{f}_{e x t ; \mathbf{x}_{n}}+\mathbf{G}_{\mathbf{x}_{n}}^{\top} \boldsymbol{\kappa}_{n}\right)
\end{aligned}
$$

such that

$$
\begin{aligned}
& \frac{\partial \ddot{\mathbf{x}}_{n+1}}{\partial \mathbf{x}_{n+1}}=\frac{1-\alpha_{m}}{\Delta t^{2} \beta\left(1-\alpha_{f}\right)}=\beta^{\prime} \mathbf{I} \\
& \frac{\partial \dot{\mathbf{x}}_{n+\mathbf{1}}}{\partial \mathbf{x}_{n+1}}=\frac{\gamma}{\Delta t \beta}=\gamma^{\prime} \mathbf{I}
\end{aligned}
$$

as before. However, the internal force vector according to GEMM is

$$
\left(1-\alpha_{f}\right) \mathbf{f}_{i n t ; \mathbf{x}_{n+1}}+\alpha_{f} \mathbf{f}_{i n t ; \mathbf{x}_{n}}=\mathbf{f}_{i n t ; \mathbf{x}_{G E M M}}
$$

so remembering that $\mathbf{f}_{i n t}=\mathbf{0}$ for all $\mathbf{q}$, since the links are considered rigid, inelastic, bodies,

$$
\begin{aligned}
\mathbf{M}_{\mathbf{x}_{n+1}} & \left(\left(1-\alpha_{f}\right) \ddot{\mathbf{x}}_{n+1}+\alpha_{f} \ddot{\mathbf{x}}_{n}\right)=-\alpha_{f} \mathbf{I}_{T}\left(\mathbf{C}_{\mathbf{x}_{n}} \dot{\mathbf{x}}_{n}+\mathbf{K} \mathbf{x}_{n}-\mathbf{f}_{e x t ; \mathbf{x}_{n}}+\mathbf{G}_{\mathbf{x}_{n}}^{\mathbf{T}} \boldsymbol{\kappa}_{n}\right) \\
& -\mathbf{f}_{i n t ; \mathbf{x}_{G E M M}}-\left(1-\alpha_{f}\right)\left(\mathbf{C}_{\mathbf{x}_{n+1}} \dot{\mathbf{x}}_{n+1}+\mathbf{K} \mathbf{x}_{n+1}-\mathbf{f}_{e x t ; \mathbf{x}_{n+1}}+\mathbf{G}_{\mathbf{x}_{n+1}}^{\mathbf{}} \boldsymbol{\kappa}_{n+1}\right)
\end{aligned}
$$

Therefore, the residual equations for the motion of the hinges and the elastic blade 
combined, including the constraints, are

$$
\begin{aligned}
\mathbf{r}_{\mathbf{x}} \equiv \mathbf{M}_{\mathbf{x}_{n+1}} & \left(\left(1-\alpha_{f}\right) \ddot{\mathbf{x}}_{n+1}+\alpha_{f} \ddot{\mathbf{x}}_{n}\right)-\mathbf{f}_{i n t ; \mathbf{x}_{G E M M}} \\
& -\left(1-\alpha_{f}\right)\left(\mathbf{C}_{\mathbf{x}_{n+1}} \dot{\mathbf{x}}_{n+1}+\mathbf{K} \mathbf{x}_{n+1}-\mathbf{f}_{e x t ; \mathbf{x}_{n+1}}+\mathbf{G}_{\mathbf{x}_{n+1}}^{\top} \boldsymbol{\kappa}_{n+1}\right) \\
& -\alpha_{f} \mathbf{I}_{T}\left(\mathbf{C}_{\mathbf{x}_{n}} \dot{\mathbf{x}}_{n}+\mathbf{K} \mathbf{x}_{n}-\mathbf{f}_{e x t ; \mathbf{x}_{n}}+\mathbf{G}_{\mathbf{x}_{n}}^{\top} \boldsymbol{\kappa}_{n}\right)
\end{aligned}
$$

and

$$
\mathbf{r}_{\kappa} \equiv \phi_{\kappa}=\left\{\begin{array}{l}
\phi_{\mathrm{q}} \\
\phi_{\mathrm{d}}
\end{array}\right\}=0
$$

Linearizing the residual equations on $\mathbf{x}$ result in

$$
\begin{aligned}
\mathbf{r}_{\mathbf{x}, \mathbf{x}} & =\left[\frac{\partial \mathbf{M}_{\mathbf{x}_{n+1}}}{\partial \mathbf{x}_{n+1}}\left(\left(1-\alpha_{f}\right) \ddot{\mathbf{x}}_{n+1}+\alpha_{f} \ddot{\mathbf{x}}_{n}\right)+\mathbf{M}_{\mathbf{x}_{n+1}}\left(1-\alpha_{f}\right) \beta^{\prime}\right]+\frac{\partial \mathbf{f}_{i n t ; \mathbf{x}_{G E M M}}}{\partial \mathbf{x}_{n+1}} \\
& +\left[\frac{\partial \mathbf{C}_{\mathbf{x}_{n+1}}}{\partial \mathbf{x}_{n+1}}\left(1-\alpha_{f}\right) \dot{\mathbf{x}}_{n+1}+\mathbf{C}_{\mathbf{x}_{n+1}}\left(1-\alpha_{f}\right) \gamma^{\prime}+\alpha_{f} \frac{\partial \mathbf{I}_{T}}{\partial \mathbf{x}_{n+1}} \mathbf{C}_{\mathbf{x}_{n}} \dot{\mathbf{x}}_{n}\right]+\left(1-\alpha_{f}\right) \mathbf{K} \\
& +\alpha_{f} \frac{\partial \mathbf{I}_{T}}{\partial \mathbf{x}_{n+1}} \mathbf{K}-\left(1-\alpha_{f}\right) \frac{\partial \mathbf{f}_{e x t ; n+1}}{\partial \mathbf{x}_{n+1}}-\alpha_{f} \frac{\partial \mathbf{I}_{T}}{\partial \mathbf{x}_{n+1}} \mathbf{f}_{e x t ; n}+\left(1-\alpha_{f}\right) \frac{\partial \mathbf{G}_{\mathbf{x}_{n+1}}^{\top}}{\partial \mathbf{x}_{n+1}} \boldsymbol{\kappa}_{n+1} \\
& +\alpha_{f} \frac{\partial \mathbf{I}_{T}}{\partial \mathbf{x}_{n+1}} \mathbf{G}_{\mathbf{x}_{n}}^{\top} \boldsymbol{\kappa}_{n},
\end{aligned}
$$

which, if it is assumed that the mass matrix is roughly constant over the time-step, leads to

$$
\begin{aligned}
\mathbf{r}_{\mathbf{x}, \mathbf{x}}= & \mathbf{M}_{\mathbf{x}_{n+1}}\left(1-\alpha_{f}\right) \beta^{\prime}+\mathbf{K}_{T ; G E M M}+\left(1-\alpha_{f}\right) \mathbf{K}+\frac{\partial \mathbf{C}_{\mathbf{x}_{n+1}}}{\partial \mathbf{x}_{n+1}}\left(1-\alpha_{f}\right) \dot{\mathbf{x}}_{n+1} \\
& +\mathbf{C}_{\mathbf{x}_{n+1}}\left(1-\alpha_{f}\right) \gamma^{\prime}-\left(1-\alpha_{f}\right) \frac{\partial \mathbf{f}_{e x t ; \mathbf{x}_{n+1}}}{\partial \mathbf{x}_{n+1}}+\left(1-\alpha_{f}\right) \frac{\partial \mathbf{G}_{\mathbf{x}_{n+1}}^{\top}}{\partial \mathbf{x}_{n+1}} \boldsymbol{\kappa}_{n+1}=\mathbf{K}_{T_{d y n} \mathbf{x x}}
\end{aligned}
$$


and

$$
\mathbf{r}_{\kappa, \mathbf{x}}=\mathbf{G}_{\mathbf{x}_{n+1}}=\mathbf{K}_{T_{d^{\prime} n_{\kappa \mathbf{x}}}}
$$

Linearizing the residual equations with respect to the constraints yields

$$
\mathbf{r}_{\mathbf{x}, \kappa}=\left(1-\alpha_{f}\right) \mathbf{G}_{\mathbf{x}_{n+1}}^{\top}=\mathbf{K}_{T_{d y n_{\mathbf{x} \kappa}}}
$$

and

$$
\mathbf{r}_{\kappa, \kappa}=0=\mathbf{K}_{T_{d y n \kappa \kappa}}
$$

Therefore, the linearized equations of motion, with constraints, are

$$
\left[\begin{array}{ll}
\mathbf{K}_{T_{\mathrm{dyn}} \times x} & \mathbf{K}_{T_{\mathrm{dyn}} \times \kappa} \\
\mathbf{K}_{T_{\mathrm{dyn}} \kappa \mathrm{x}} & \mathbf{K}_{T_{\mathrm{dyn}} \kappa \kappa}
\end{array}\right] \Delta\left\{\begin{array}{c}
\mathbf{x} \\
\kappa
\end{array}\right\}=-\mathbf{r}
$$

Additionally, the Bottasso [118] transformation scales the Lagrange multipliers to improve the matrix condition number, which is not shown, but it is the same procedure as in the previous section. The solution to this system is the same as outlined in the continuum mechanics section, using the Newton-Raphson procedure. 


\section{Chapter 5}

\section{Numerical Experimentation}

This chapter contains the verification and validation of the model described previously, after a brief description of the program. The verification is on a program function level first, to check the convergence of the fore-shortening effect on cantilevered beams, of a four-bar mechanism, and of the inflow for an experimental blade. A comparison to validate each component follows their verification, using the same three examples. After the components are verified and validated, the complete program is verified for a general hinge arrangement attached to an approximate SHARCS blade. The simulated SHARCS blade is substantiated with experimental data from whirltower tests at the National Research Council of Canada (NRC). A comparison of forward flight motion with the control active and inactive is shown.

\subsection{Program Description}

The main program, b3dsm.m (Blade 3-D Structural Model), is a procedural program run in MATLAB that flows according to Figure 5.1. The complete program can be found on the DVD that accompanies this thesis. The commented header of each MATLAB m-file explains the purpose of its following computer code. Normal use of 
the program is described next, with the caveat that some of the functions, or command lines, were removed (commented out) for verification and validation purposes, which is mentioned in the appropriate section.

Once a user starts the code, the program checks for a save file containing the description of the hinges, with the DH parameters already saved. This process saves time if the link model has not changed, as the link geometry and the derivative matrices are independent of the initial conditions. If the hinges have not been saved, or the DH parameter spreadsheet has been changed, then the program initiates the procedure for calculating the geometric properties, the link masses, and the constraint equation for the articulation.

The hinge orientation is calculated next, such that a user can specify to lock or unlock hinges, depending on the desired initial geometry. (For example, if the user wishes to keep commanded collective and cyclic angles, they could specify those hinges, and solve for the remaining joints to ensure a closed geometry.) Based on Leishman [3], the root pitch angle $\theta_{0}$ is solved, using trimmed conditions in hover, by estimating the coefficient of thrust for its current value and iterating between the two parameters.

Once the program has the hinge geometry stored in memory, the elastic blade geometry is determined, which is a function of the aerodynamic chain orientation and position. At this time, the constraint coupling between the blade and the rigid body articulation is calculated also. The mesh is applied to the blade in such a way that the node numbering begins at the trailing edge at the root, in the $y$-direction, then the $z$ - and $x$-directions. This improves the skyline of the mass, stiffness, and damping matrices. Unless pre-loaded, there should not be any residual stress within the elastic model.

The external forces, from aerodynamics, gravity, and control loads, are calculated 


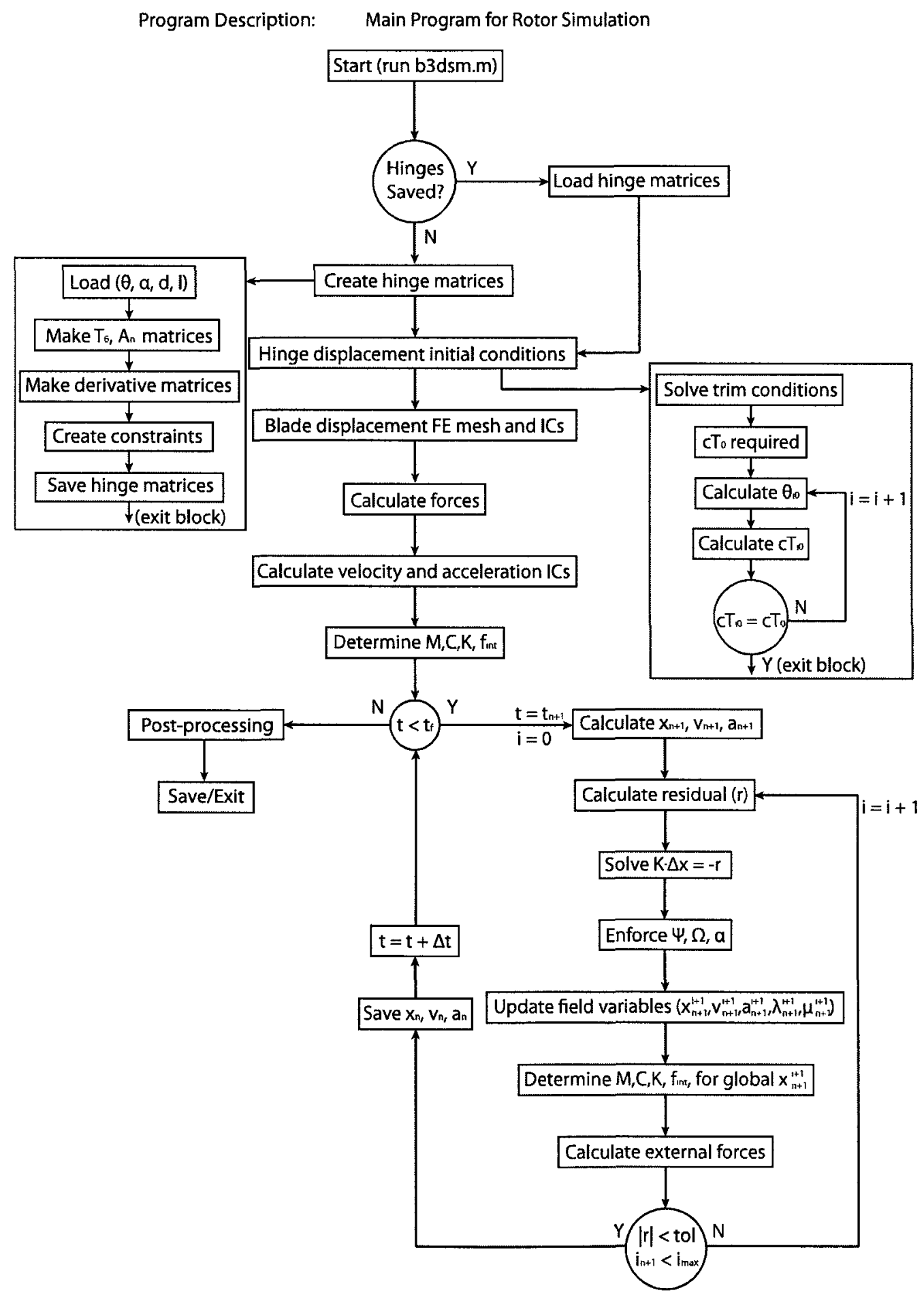

Figure 5.1: Flow-chart of the main program. 
next, and are used to calculate the initial velocity and accelerations of both the articulation and the rotor blade. The time-marching solution begins after this point in the program.

The residual vectors, based on Equations (4.64) and (4.65), are determined along with the dynamic tangential stiffness matrix, based on Equations (4.67-4.70). The code solves the system using Equation (4.71), and the displacements, velocities, and accelerations are updated using the GEMM and procedure proposed in Section 4.4.2.

Finally, after the field variables are updated, the program re-calculates the equation of motion matrices, external forces, including the control forces, and finds the new residual vector. If the residuum is smaller than the user-specified tolerance, the next time step is prepared. Once the program time runs to the final time, or a specified number of iterations is reached that means the configuration did not converge, the variables are saved, and the program stops.

\subsection{Verification and Validation of Model Subcom- ponents}

The elastic code, rigid body code, and aerodynamic code segments are verified and validated next. The elastic code was verified by comparing increasingly finer material meshes to one another, and validated by comparing them to another beam model and foreshortening equations. The verification and validation of the rigid body articulation is performed by comparing it to a different technique used by Blajer [71].

The nonlinear inflow model is compared to NASA experiments [86-88], but the inflow model is not based on finite elements or finite differences, but instead it is a Fourier series, so the process and final results are corroborated, but no verification is necessary. The control law was also correlated to experimental results in Harold's 
dissertation [8], so that has not been repeated here.

\subsubsection{Elastic Blade Foreshortening Effect}

To verify the elastic beam model, the articulation is removed by disabling the joint variables and setting the link lengths to zero, and the external forces from the control and aerodynamics are not included. Doing so reduces the elastic model to a nonlinear cantilevered elastic three-dimensional beam, which ensures that only the elastic effects affect the solution.

To check the convergence and the accuracy of the elastic model, a comparison of the foreshortening effect is provided. Foreshortening is the nonlinear effect that arises from beam bending, as Figure 5.2 shows. As the beam deflects from its undeformed position due to a transverse load, the foreshortening effect is the contraction of the longitudinal axis projected length. The effect can only be analysed with a nonlinear elastic model, hence, it is used to show the accuracy of the nonlinear model.

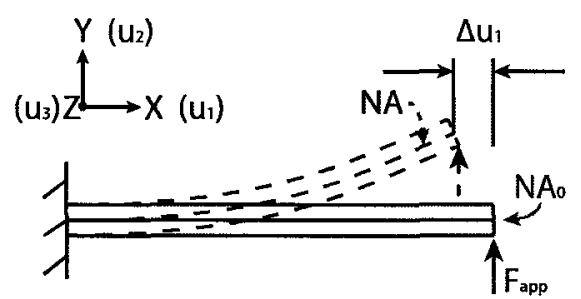

Figure 5.2: Foreshortening effect on a three-dimensional beam, compared at the neutral axis (NA).

These beam models use the following material properties, shown in Table 5.1, and the rationale for using these parameters is to compare the model with validated results next.

The momentum tolerance, the norm of the residual divided by the norm of the 
Table 5.1: Properties of the example elastic comparison beam.

\begin{tabular}{lclc}
\hline Property & Value & Property & Value \\
\hline Length (x-axis) & $1.0[\mathrm{~m}]$ & Density & $1770\left[\frac{\mathrm{kg}}{\mathrm{m}^{3}}\right]$ \\
Chord (y-axis) & $0.2[\mathrm{~m}]$ & Young's Modulus & $1.792 \times 10^{4}[\mathrm{GPa}]$ \\
Thickness (z-axis) & $0.1[\mathrm{~m}]$ & Poisson's Ratio & 0.3 \\
\hline
\end{tabular}

force, for these simulations is $1 \times 10^{-8}$. The displacement tolerance is also calculated, which is calculated based on the root-mean-square of the difference in solutions between the $i^{\text {th }}$ and $i+1^{\text {th }}$ iteration, and is of the same order of magnitude.

The model under scrutiny is for an isotropic, homogeneous beam and in the current work, without any numerical damping, $\rho_{\infty}=1.0$. The beam is centred to the inertial frame of reference, and there is no twist along its length. It is loaded with a force of $F_{a p p}=100 \mathrm{kN}$ at the centre of the cross-section at the end of the beam, in the $y$-direction.

As can be seen from Figure 5.3, the displacement field converges to a solution with only a few elements. The percent difference of the tip displacement, where the difference should be the largest, based on the number of elements, is shown in Table 5.2. This table shows good convergence to the 10 element model, chosen as a reference, particularly in the $x$ - and $y$-directions. The $z$-direction displacement is on the order of machine epsilon, $\epsilon \approx 10^{-16}$.

Table 5.2: Percent difference verification of tip displacement in a cantilevered beam compared to 10 element model.

\begin{tabular}{cccccc}
\hline Direction & 2 nele & 3 nele & 5 nele & 10 nele & $10 \times 2$ nele \\
\hline$u_{x}[m]\left(\times 10^{-10}\right)$ & 4.38 & 4.59 & 4.72 & 4.80 & 4.84 \\
$u_{y}[m]\left(\times 10^{-5}\right)$ & 2.68 & 2.76 & 2.80 & 2.83 & 2.84 \\
$u_{z}[m]\left(\times 10^{-15}\right)$ & 0.67 & -1.12 & 0.96 & 1.35 & -4.15 \\
\hline
\end{tabular}



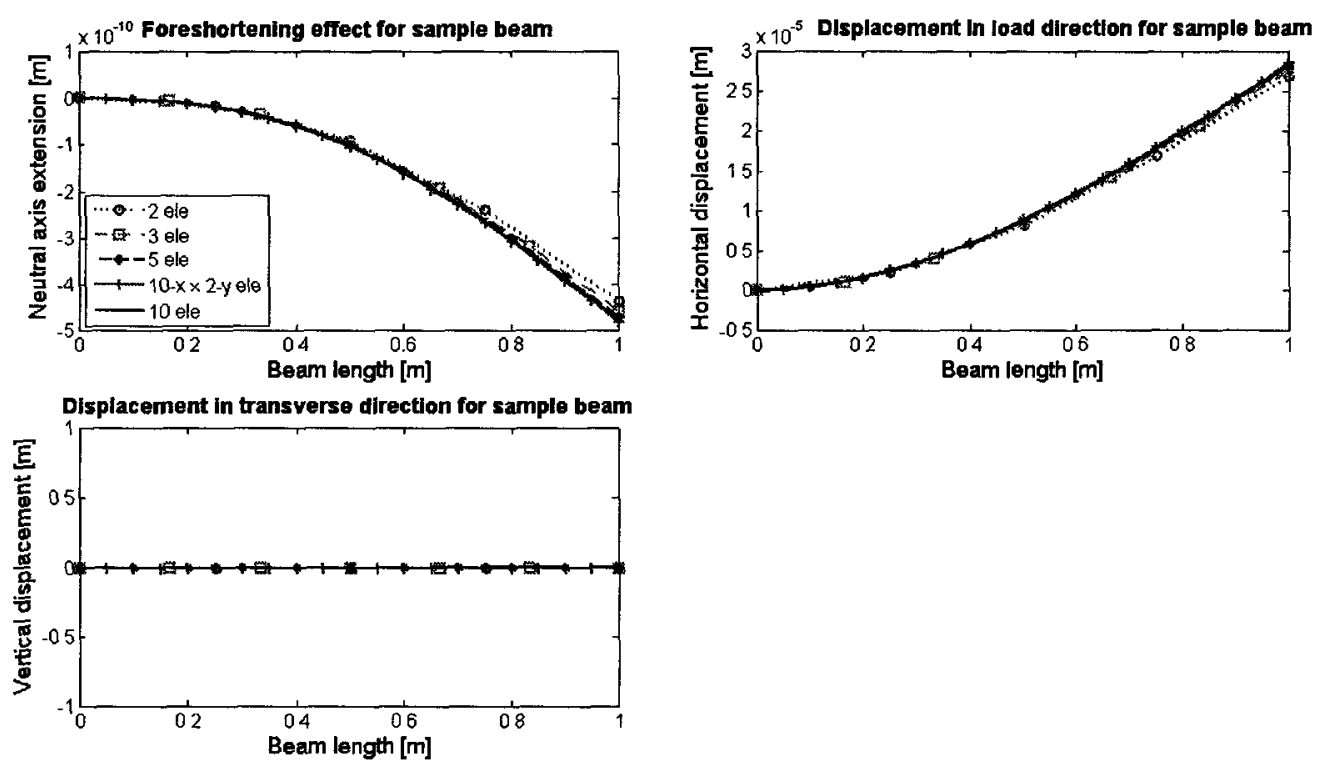

Figure 5.3: Distribution of displacement along the beam neutral axis from continuum statics approach with multiple elements in the $\mathrm{x}$-direction, one element in $\mathrm{y}$ - and z-directions (except listed).

Because the elastic model has the potential for a great number of degrees of freedom, whereas the articulation model has fewer in general, the time for each solution was also recorded. For the static model, the solution time, printed by MATLAB, is for two Newton-Raphson iterations, and is shown in Table 5.3. As is clear from the table, the cost of having multiple elements in the chordwise or thickness directions is high.

Table 5.3: Time for static solution of multiple elements for cantilevered threedimensional beam.

2 elements 3 elements 5 elements 10 elements $10 \times 2$ elements

\begin{tabular}{llllll}
\hline Time $[\mathrm{s}]$ & 1.1 & 1.3 & 1.8 & 3.4 & 26.1 \\
\hline
\end{tabular}

Figure 5.4 shows the foreshortening effect and perpendicular directions by Ghorashi [119], which was used to validate his results. Figure 5.5 shows the foreshortening 
effect and orthogonal directions using the current three-dimensional model. The circles in the figure by Ghorashi represent the nonlinear equation for the foreshortening effect; both his and this work matches the theory. In this case, both models show the foreshortening effect clearly, with the same magnitude and curve.
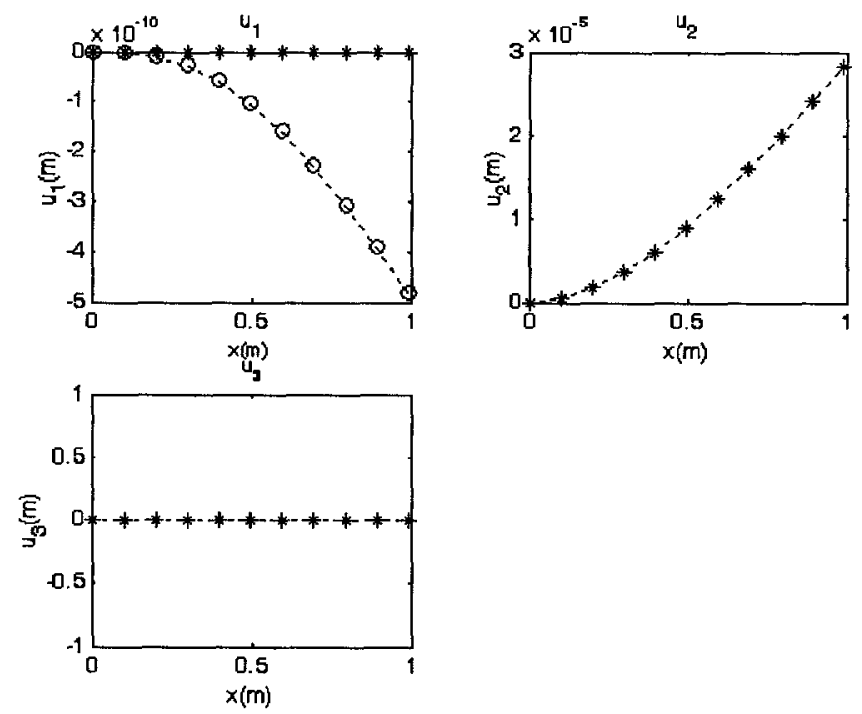

Figure 5.4: Distribution of displacement components along the beam with linear static $\left(^{*}\right)$, nonlinear static $(-)$ and foreshortening equation (o) [119].

The neutral axis set of nodes was used to examine the foreshortening effect, because the deflection of the edges of the beam is orders of magnitudes higher than the foreshortening effect. The model in Figure 5.5 has 10 longitudinal elements, and 1 chordwise element. Even with a very coarse grid the foreshortening effect is clear, with identical magnitudes. Note that although distinct points are plotted in MATLAB joined by line segments, the actual curves between the points are quadratic, so the element points are $\mathcal{C}^{2}$ continuous.

The elastic bending can also be compared to linear theory, in which formulae for deflections are well-known. For a point load applied at the end of a cantilevered 

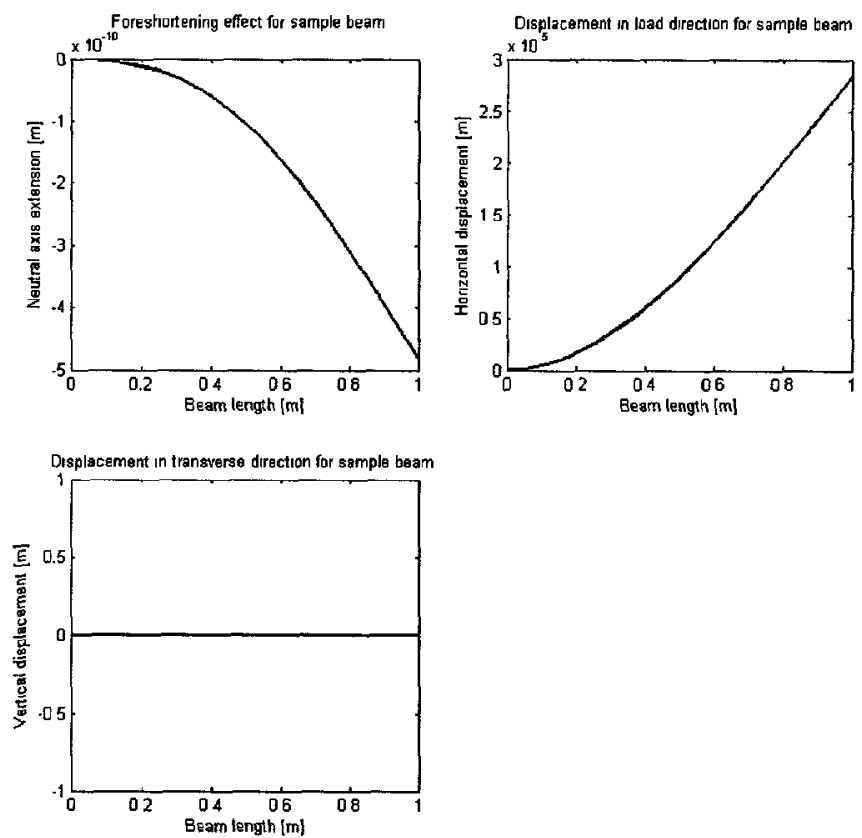

Figure 5.5: Beam displacement with nonlinear continuum mechanics with 10 elements.

beam, the formula for tip deflection is

$$
\delta=\frac{F_{a p p} L^{3}}{3 E I}
$$

in which $\delta$ is the displacement in the direction of the loading $F_{a p p}$. Since these values are all known for the current problem, the linear tip displacement is $\delta=$ $2.79 \times 10^{-5} \mathrm{~m}$. The centreline tip displacement in the $y$-direction, using 10 elements, is $\delta=2.82 \times 10^{-5} \mathrm{~m}$, which gives a percent difference of only $1.14 \%$. Of course, because the beam formula is for linear elastic material, it should be accurate, but it may not match exactly. 


\subsubsection{Four-bar Mechanism}

A crank-rocker, as in Figure 5.6), is a closed-kinematic chain four-bar mechanism whose motion can be solved analytically or using parallel kinematic methods. Therefore, it is an ideal candidate to showcase the accuracy of the generalized- $\alpha$ method modified by the method outlined by Arnold and Brüls. (Note, because the elastic part is not present, it is technically not GEMMAB, but a generalized- $\alpha$ method with an acceleration modification. Here that time discretization is referred to as Gen- $\alpha-A B$.)

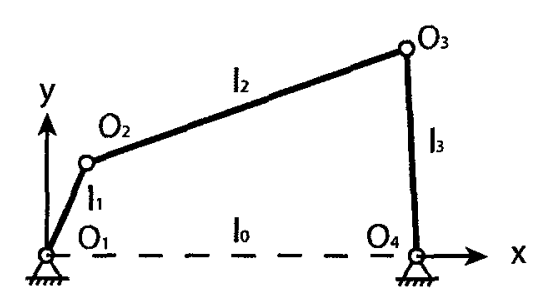

a) Four-bar mechanism

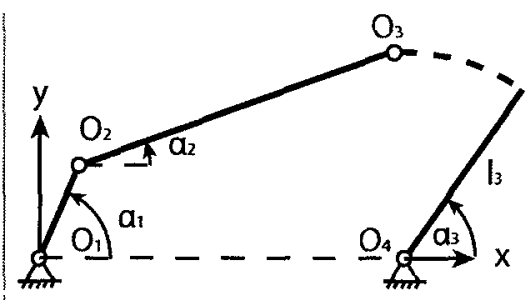

b) Open-loop representation

Figure 5.6: A four bar mechanism with the open loop representation, cut at the end of the second link.

The four-bar mechanism (in this case, a crank-rocker) and its properties are in Table 5.4, and the DH parameterization of the links is shown in Table 5.5:

Table 5.4: Physical properties of the four-bar mechanism.

\begin{tabular}{ccccc} 
Label [] & $l_{0}$ & $l_{1}$ & $l_{2}$ & $l_{3}$ \\
Length [m] & 1.0 & 0.3 & 1.0 & 0.6 \\
Mass [kg] & 0 & 1.5 & 5.0 & 3.0 \\
\hline
\end{tabular}

The four-bar mechanism tests are done with the elastic model turned off, that is, the elastic degrees of freedom are eliminated, so that they do not affect the solution. There are no aerodynamic or control forces, or any external torques either. To verify the Gen- $\alpha$-AB method, the comparison in Figures 5.7 and 5.8 show the effect of 
Table 5.5: DH parameterization of the four-bar mechanism.

\begin{tabular}{ccccc|cccccc}
\hline \multicolumn{4}{c}{ Chain 1 } & \multicolumn{1}{c}{ Chain 2 } \\
\hline Link \# & $\theta_{n}$ & $d_{n}$ & $a_{n}$ & $\alpha_{n}$ & Link \# & $\theta_{n}$ & $d_{n}$ & $a_{n}$ & $\alpha_{n}$ \\
\hline $\mathrm{A} 1$ & $\alpha_{1}$ & 0 & $l_{1}$ & 0 & $\mathrm{~A} 3$ & 0 & 0 & $l_{0}$ & 0 \\
$\mathrm{~A} 2$ & $\alpha_{2}$ & 0 & $l_{2}$ & 0 & $\mathrm{~A} 4$ & $\alpha_{3}$ & 0 & $l_{3}$ & 0 \\
\hline
\end{tabular}

changing $\Delta t$. The initial conditions for this test are based on $\alpha_{10}=\frac{\pi}{2}$ and $\dot{\alpha}_{10}=1 \mathrm{~s}^{-1}$, and the program automatically solves for the remaining hinge geometry.
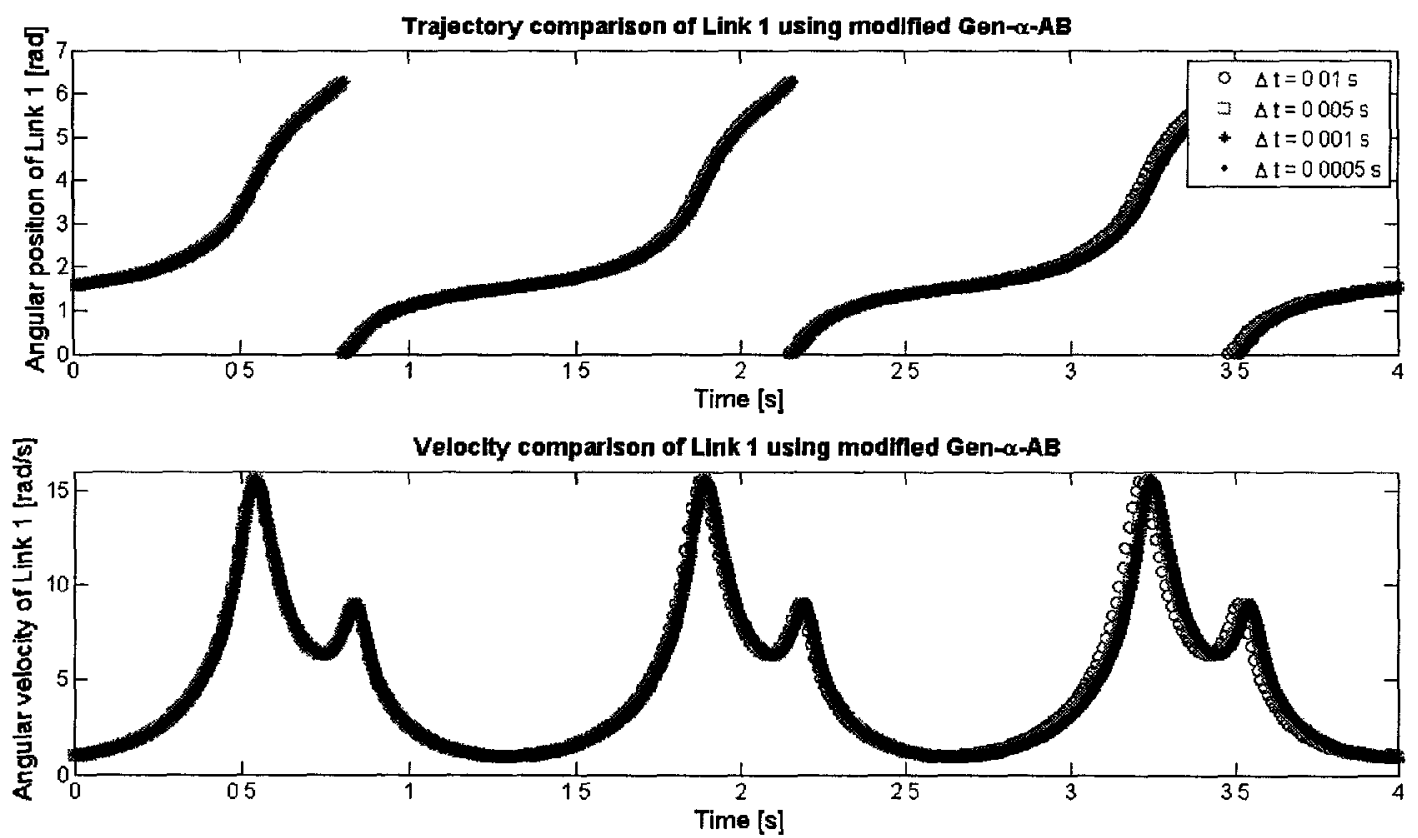

Figure 5.7: The position solution to the 4-bar mechanism using the Gen- $\alpha-A B$ method with different time-steps.

Figure 5.7 shows the displacements and velocities of Frame 1, which is the actuated joint. Figure 5.8 shows the constraint forces using the modified method, to ensure that the displacement, velocity, and accelerations are not the only parameters matched properly. The periods of the cycle are compared in Table 5.6, which shows the percent difference based on the smallest time-step. 

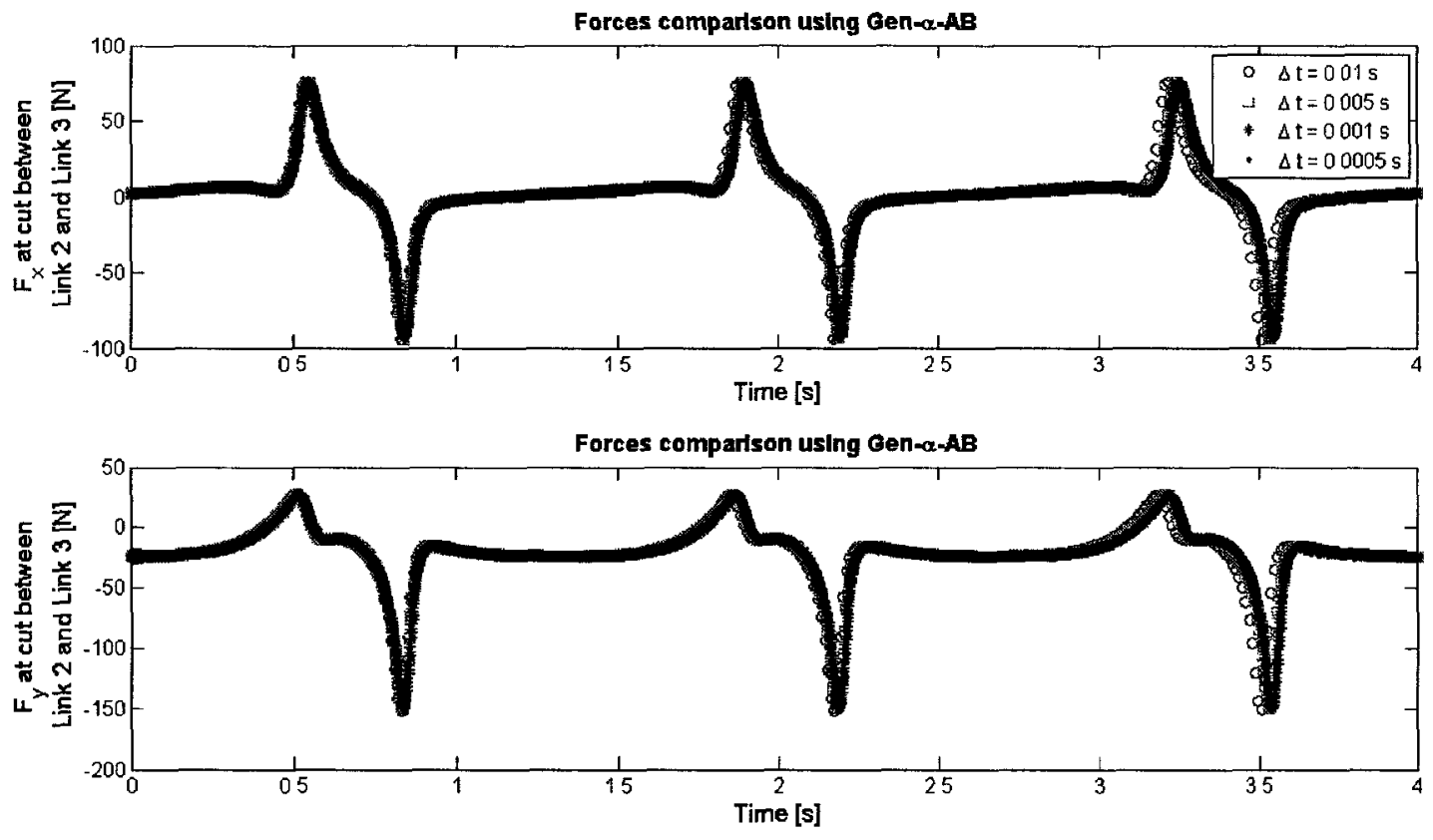

Figure 5.8: The constraint forces using Gen- $\alpha-\mathrm{AB}$ methods combined.

Table 5.6: Period convergence of the 4-bar mechanism.

\begin{tabular}{ccccc}
\hline & $\Delta t=0.01 \mathrm{~s}$ & $\Delta t=0.005 \mathrm{~s}$ & $\Delta t=0.001 \mathrm{~s}$ & $\Delta t=0.0005 \mathrm{~s}$ \\
\hline Period [s] & 1.34 & 1.35 & 1.35 & 1.35 \\
$\%$ diff. [\%] & 0.98 & 0.16 & 0.07 & N $/ \mathrm{A}$ \\
\hline
\end{tabular}

The simulation carried out by Blajer [71] was performed with the same initial conditions, using Runge-Kutta with a very small step size ( $\Delta t=0.0005 \mathrm{~s})$ to obtain a reference solution. He then compared it to the velocity-stabilization method, using the velocity to prevent constraint drift. That work was reproduced for this dissertation, to determine the efficacy of the stabilization method, and to compare it to the Gen$\alpha$-AB method to validate the parallel kinematics sub-program.

This method, with $\Delta t=0.0005 \mathrm{~s}$, takes approximately $17.4 \mathrm{~s}$ to complete. Comparatively, the velocity drift method takes much longer, for the same time interval the solution time is $84.2 \mathrm{~s}$. 

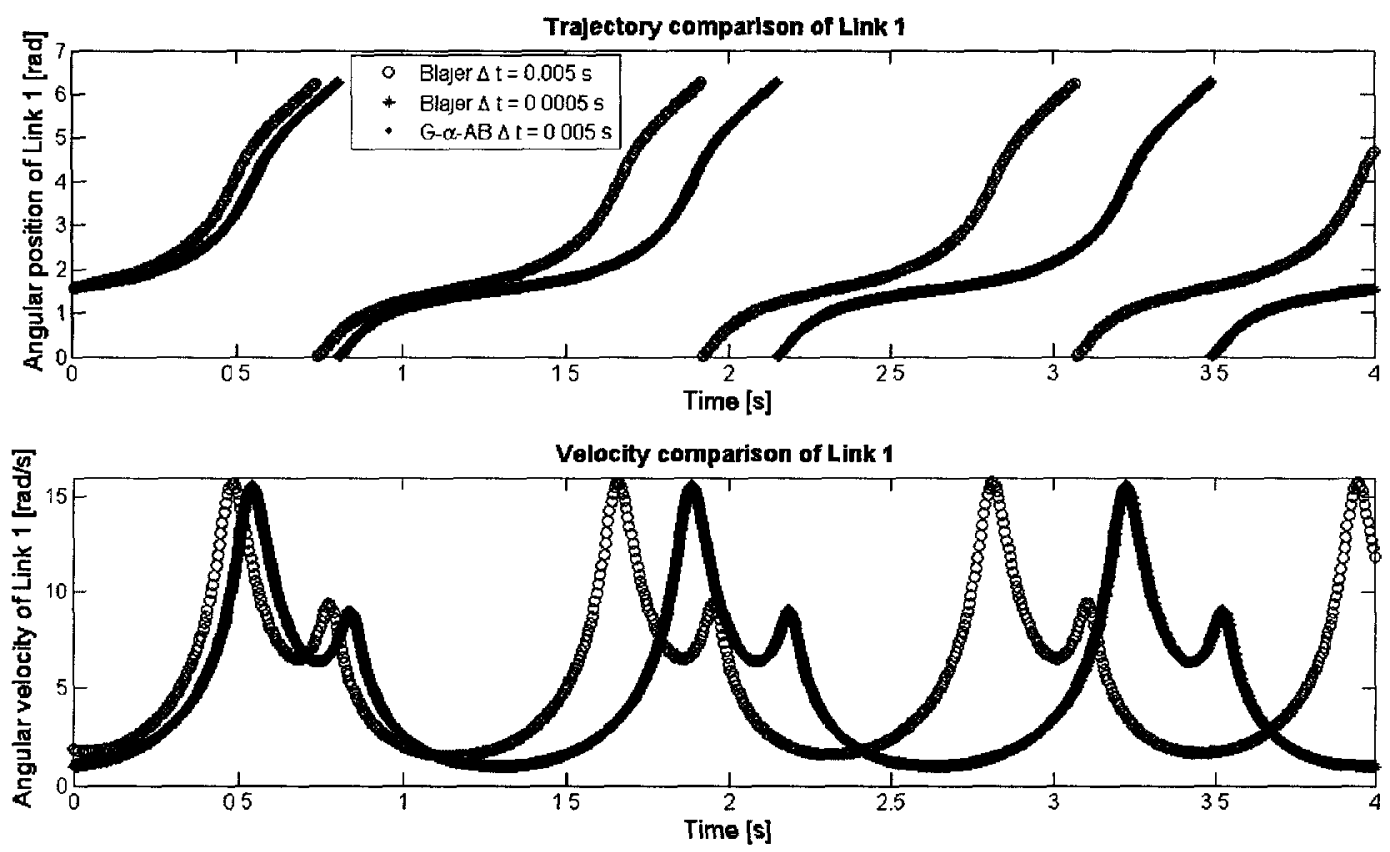

Figure 5.9: The position solution to the 4-bar mechanism using the velocity drift method (Blajer) and the Gen- $\alpha-\mathrm{AB}$ for constrained kinematics.

Note that the definition for the angles is slightly different in Blajer than with this parameter test: in Blajer, the angles are defined with respect to the inertial $x$-axis, whereas the program automatically defines the angles with respect to the previous $x$-axis, because of the $\mathrm{DH}$ characteristics. Another difference is where he virtually cuts the joints; in his work, he cuts the joint at the origin of Frame 4, attached to the ground. In this case, the velocity drift method solves faster and larger time steps are more accurate, but because such a fortuitous cut cannot be guaranteed for the general solver, the current profile is more realistic.

Blajer shows the evolution of the position and velocity of Link 1 only, in his paper. So, the comparison of the filtering method and the Gen- $\alpha-\mathrm{AB}$ method in Figure 5.9 shows the position and velocity from those two methods. As can be seen, the methods match almost exactly when $\Delta t=0.0005 \mathrm{~s}$ for the velocity drift algorithm, and $\Delta t=0.005 \mathrm{~s}$ for the modified Gen- $\alpha$ method. When the $\Delta t=0.005 \mathrm{~s}$, the 
velocity drift method the results correlate poorly, matching the general trends, but not matching the curves properly. Table 5.7 shows the period comparison between two time steps using the velocity stabilization method and with the modified generalized- $\alpha$ scheme.

Table 5.7: Crank-rocker mechanism analysis for velocity stabilization and Gen- $\alpha$-AB schemes.

\begin{tabular}{cccc}
\hline & \multicolumn{2}{c}{ Velocity filter } & Gen- $\alpha$-AB \\
\hline & $\Delta t=0.0005 \mathrm{~s}$ & $\Delta t=0.005 \mathrm{~s}$ & $\Delta t=0.005 \mathrm{~s}$ \\
\hline Period [s] & 1.34 & 1.16 & 1.35 \\
$\%$ diff. [\%] & 0.45 & 14.6 & 0 \\
\hline
\end{tabular}

\subsubsection{Aerodynamic Inflow Model Comparison to Experiment}

The program normally calculates inflow at each time step depending on the orientation and position of the rotor blade, but to test the forward inflow model compared to experimental results the subroutine was modified to show the non-dimensional inflow at specified azimuth intervals. The structural and control parts of the program are turned off, although flapping and feathering equations are used to provide the azimuthally dependent sinusoidal motions of both hinges [3].

The values for the structural and geometric properties are listed in Table 5.8, which come from the NASA experiments [86-88]. In all cases, the rotor had 4 blades, used a NACA 0012 airfoil, and had a rectangular planform. The blades are 33.88 in long and have a chord length of $2.6 \mathrm{in}$, with a linear twist of $-8^{\circ}$ and a non-dimensional root cut-out of $e=0.24$. The measurements were taken one chord length above the rotor disc, by laser Doppler velocimetry. The experiment had a mock fuselage and rotor, so the results do not correspond precisely to the theory as an isolated rotor 
Table 5.8: Properties of the aerodynamic inflow calculation and experiment [86-88].

\begin{tabular}{lccc} 
Property & $\mu=0.15$ & $\mu=0.23$ & $\mu=0.30$ \\
\hline Shaft angle, $\alpha_{s}\left[^{\circ}\right]$ & -3.00 & -3.04 & -4.04 \\
Root theta, $\theta_{0}\left[^{\circ}\right]$ & 9.37 & 8.16 & 10.31 \\
Cyclic, $\theta_{1 s}\left[^{\circ}\right]$ & 3.23 & 4.13 & 5.88 \\
Cyclic, $\theta_{1 c}\left[^{\circ}\right]$ & -1.11 & -1.52 & -1.55 \\
Coning angle, $\left[^{\circ}\right]$ & 1.50 & N/A & 2.13 \\
Thrust coeff., $c_{T}[]$ & 0.0063 & 0.0063 & 0.0065 \\
Torque coeff., $c_{Q}[]$ & 0.00036 & 0.00036 & 0.00043 \\
Airstream speed, $v_{\infty}[\mathrm{kts}]$ & 55.4 & 85.3 & 111.4 \\
\hline
\end{tabular}

would.

However, as shown in Figure 5.10, Mangler and Squire's theory, with equal weights on the loading types, accords well with the experiment. The longitudinal flow is more accurate towards the aft of the rotorcraft (the positive coordinates on the graphs), and the lateral flow is more reliable towards the edges of the disc. The upwash at the edges of the disc are captured by Mangler and Squire theory, but not by Drees or uniform inflow models.

Another observation of these examples is that as the forward velocity increases, the experiment and the theory match poorer. This can be attributed, partially, to the fact that as the advance ratio increases, the velocity field is increasingly perturbed by the rotor hub and fuselage. Magnifying this effect, because the mean inflow decreases slightly as the forward velocity increases, a larger ratio of the total inflow is affected by the hub and fuselage. 

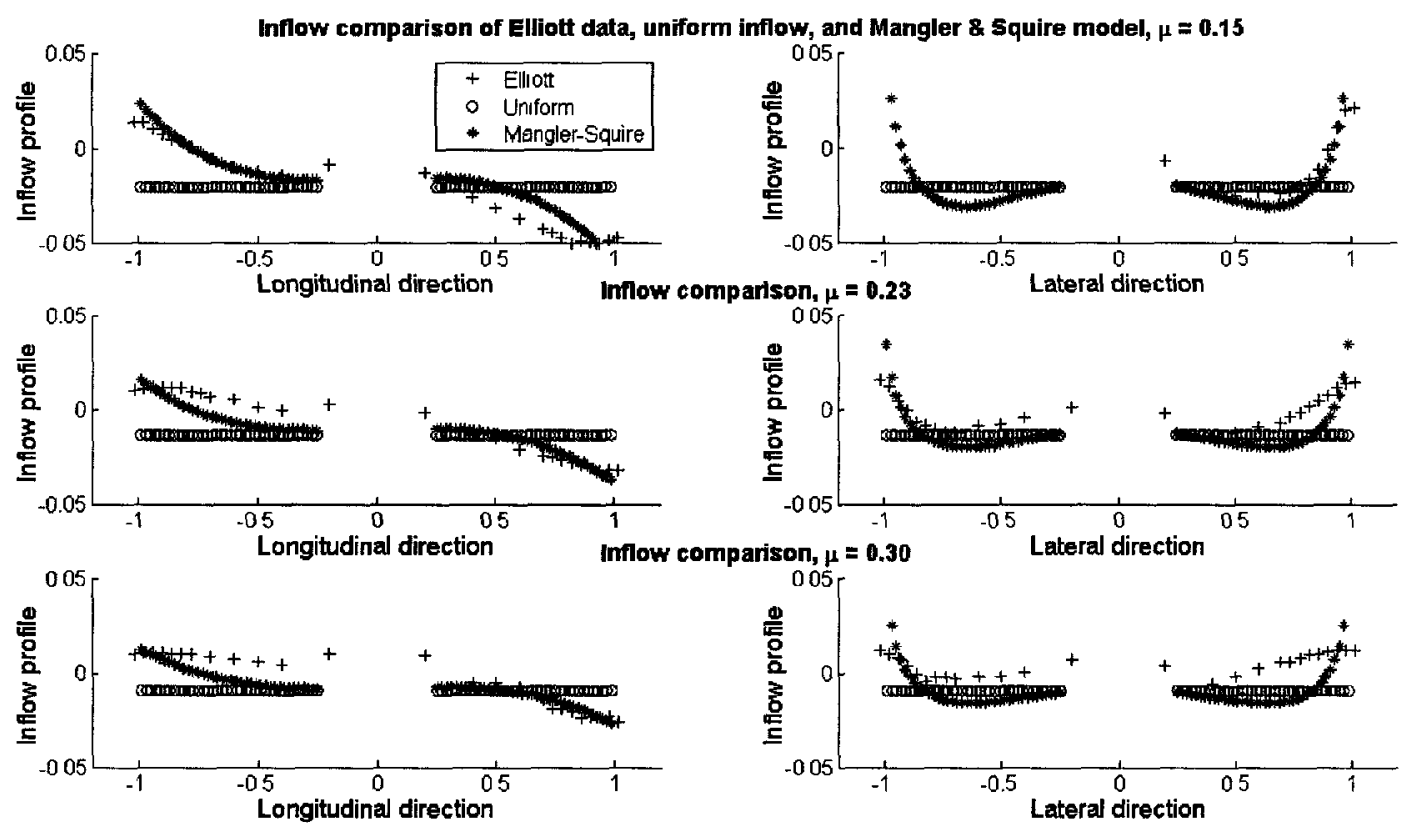

Figure 5.10: Comparison of inflow between theory and NASA experiment.

\subsection{Verification of Complete Articulated Model}

To test the convergence of the entire program, a set of structural parameters selected to emulate the SHARCS hub arrangement and the model was run for varying time steps. This test shows the convergence of the displacement parameters, the convergence of the forces at the root of the blade, the constraint forces at the virtually cut point, and the support forces required for any locked hinges (such as the azimuth angle).

The structural parameters selected for the articulation are based on the DH parameterization in the Multibody chapter. The values of the links are listed below, in Table 5.9 , and the actuator mass, which is not listed, is $0.05 \mathrm{~kg}$. (It is modelled as a separate entity, a point mass, rather than a link, based on the original design for the APL.) 
Table 5.9: Link properties for complete simulation verification.

\begin{tabular}{cccccc}
\hline Link & $\mathrm{H}$ & $\mathrm{F}$ & $L+P^{*}$ & $\mathrm{~d}$ & $P_{s x}$ \\
\hline Length $[\mathrm{m}]$ & $5.160 \mathrm{E}-02$ & $0.000 \mathrm{E}+00$ & $7.445 \mathrm{E}-02$ & $1.091 \mathrm{E}-01$ & $5.160 \mathrm{E}-02$ \\
Radius $[\mathrm{m}]$ & $1.750 \mathrm{E}-02$ & $1.750 \mathrm{E}-02$ & $1.750 \mathrm{E}-02$ & $2.580 \mathrm{E}-02$ & $0.000 \mathrm{E}+00$ \\
Density $\left[\frac{\mathrm{kg}}{\mathrm{m}^{3}}\right]$ & $7.960 \mathrm{E}+03$ & $7.960 \mathrm{E}+03$ & $7.960 \mathrm{E}+03$ & $0.000 \mathrm{E}+00$ & $0.000 \mathrm{E}+00$ \\
\hline$P_{s y}$ & $P_{L}$ & $P_{A}$ & $P_{H}$ & $P_{O}$ & $P_{L_{2}}$ \\
\hline $3.985 \mathrm{E}-02$ & $1.120 \mathrm{E}-01$ & $3.810 \mathrm{E}-02$ & $3.985 \mathrm{E}-02$ & $3.635 \mathrm{E}-02$ & $1.120 \mathrm{E}-01$ \\
$0.000 \mathrm{E}+00$ & $6.350 \mathrm{E}-03$ & $6.350 \mathrm{E}-03$ & $6.350 \mathrm{E}-03$ & $1.750 \mathrm{E}-02$ & $0.000 \mathrm{E}-00$ \\
$0.000 \mathrm{E}+00$ & $7.960 \mathrm{E}+03$ & $7.960 \mathrm{E}+03$ & $7.960 \mathrm{E}+03$ & $0.000 \mathrm{E}+00$ & $0.000 \mathrm{E}+00$ \\
\hline
\end{tabular}

* Wrist rotation, therefore, in kinematic equations, two lengths always summed

The initial configuration of the hinges depends on the user constraints; for example, locking certain hinges can cause degeneracy in the articulation or cause negative $c_{T}$ in the aerodynamics, which causes errors in the inflow calculation. For the test cases, degeneracy is avoided in the initial conditions, ensured by the loop closure equations that the program runs, and simulating trimmed, hover flight. The results shown for verification purposes have preset hinges for the aerodynamic chain, the swashplate tilt and collective, and the pitch link and its actuator. The values of these hinges is listed in Table 5.10, and Figure 5.11 shows the initial geometry of the articulation.

Table 5.10: Hinge initial conditions, locked by user, based on trim hover conditions.

\begin{tabular}{cccccccc}
\hline Hinge & $\psi$ & $\beta$ & $\theta$ & $\theta_{1 s}$ & $\theta_{1 c}$ & $P_{L}$ & $P_{L_{2}}$ \\
\hline Angle $\left[{ }^{\circ}\right]$ & 0 & 1.5 & $\theta_{0}^{*}$ & 0 & 0 & & \\
Length [m] & & & & & .112 & .112 \\
\hline
\end{tabular}


With those hinges constrained, the constraint equations are run and solved to close the kinematic chains. There are also hinges locked during the simulation, to ensure that the correct motions are prescribed, such as the hub angle, all swashplate angles/displacements, the bottom spherical joint of the pitch link, and the APL itself. Therefore, only three constraint forces are required, since the system is degenerate from six degrees of freedom to three (one position and two orientation constraints).

The elastic blade is isotropic, homogeneous, is discretized by 10 longitudinal elements, and has a rectangular cross-section. However, it does have the SHARCS blade twist, which is plotted in Figure 5.12.

The simulation itself goes through a start-up phase, in which the angular velocity of the hub increases from 0 to full speed, which is approximately $168.2 \frac{\mathrm{rad}}{\mathrm{s}}$ or 1555 RPM. The equation that governs the start-up velocity is

$$
\omega=\frac{1}{2}\left(1-\cos \left(\frac{\pi t}{\tau}\right)\right) \Omega
$$

where the final rotor speed is $\Omega$, and $\tau$ is the start-up phase time. The position and acceleration can be found by taking the time integral and derivative, respectively. Once full speed is reached, the simulation runs for an analysis time of $t_{n}=0.2 \mathrm{~s}$, which is roughly 5 revolutions. Aerodynamics are included in this simulation, but for testing the agreement of these simulations, the control algorithm is not. These simulations, in preparation for the validation scenario, are close to trim conditions; during the start-up phase, trim will not be achieved, but the full speed rotor does go to equilibrium (trim).

Figures 5.13, 5.14, and 5.15 show the convergence of the full model, the angular displacements of the flap and lag, and the translation of the pitch link first; the resultant force vectors at the root next; and the constraint forces and moments last. 

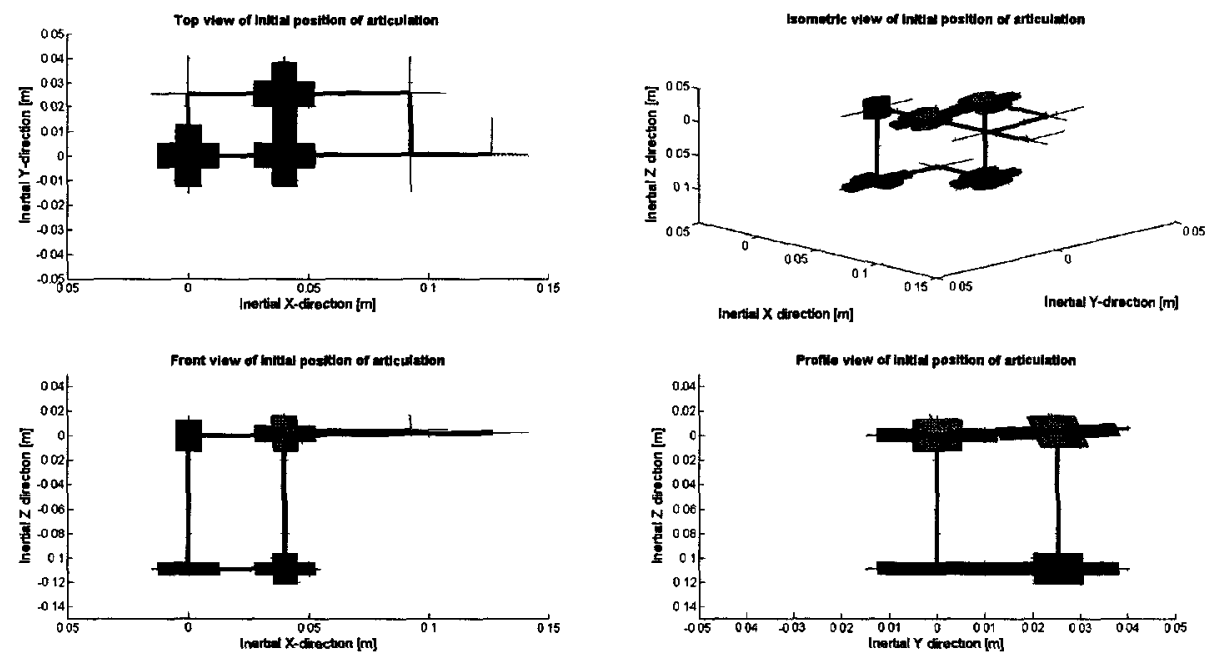

Figure 5.11: Simulated articulation in original position.

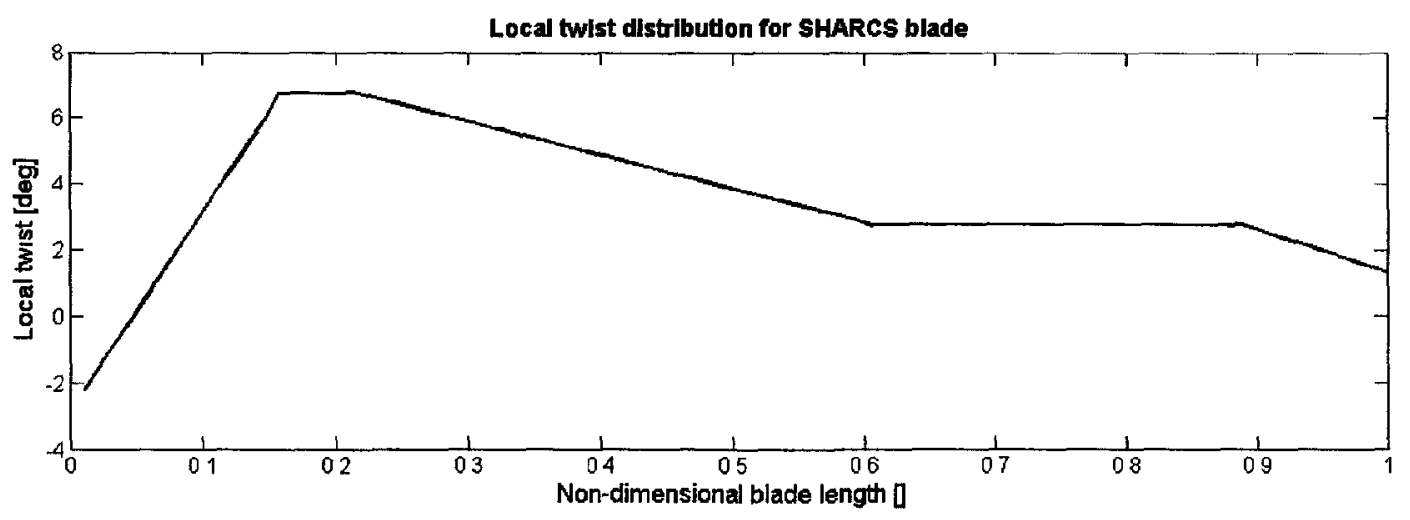

Figure 5.12: Twist distribution of SHARCS rotor blade. 
In both the displacement variables and the force variables the graphs converge quickly. In the constraint forces, there is some incongruous variation at the end of the start-up phase. This is likely caused by the tangential deceleration of the hub, because there is jerk during the start-up phase, equivalent to $-\left(\frac{\pi}{\tau}\right)^{2} \frac{\Omega}{2}$, which is substantial since $\tau$ in these simulations is typically very small.
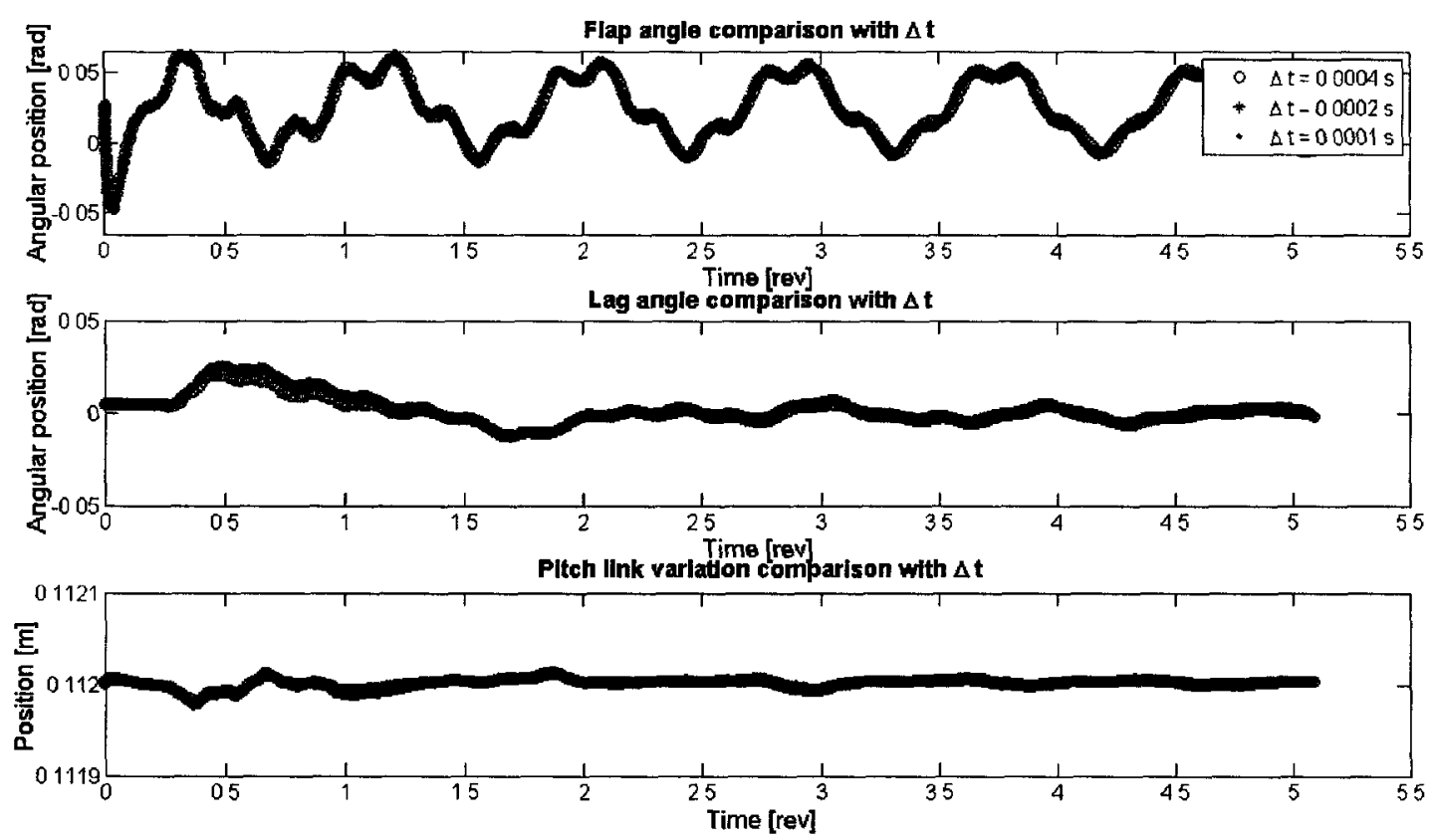

Figure 5.13: Displacements of flap, lag, and pitch link under trim hover aerodynamic load.

\subsubsection{Second-Order Convergence of Full Model}

Finally, to verify the model, a time convergence study is included. The model theory dictates that the time convergence should be second order; however, as Figure (5.16) seems to indicate, only linear convergence is acheived for the varying time steps.

To generate this plot, the nominal start up phase was used, but only the time at $t=0.01 \mathrm{~s}$ was analysed. The norm of the displacement along the neutral axis was 

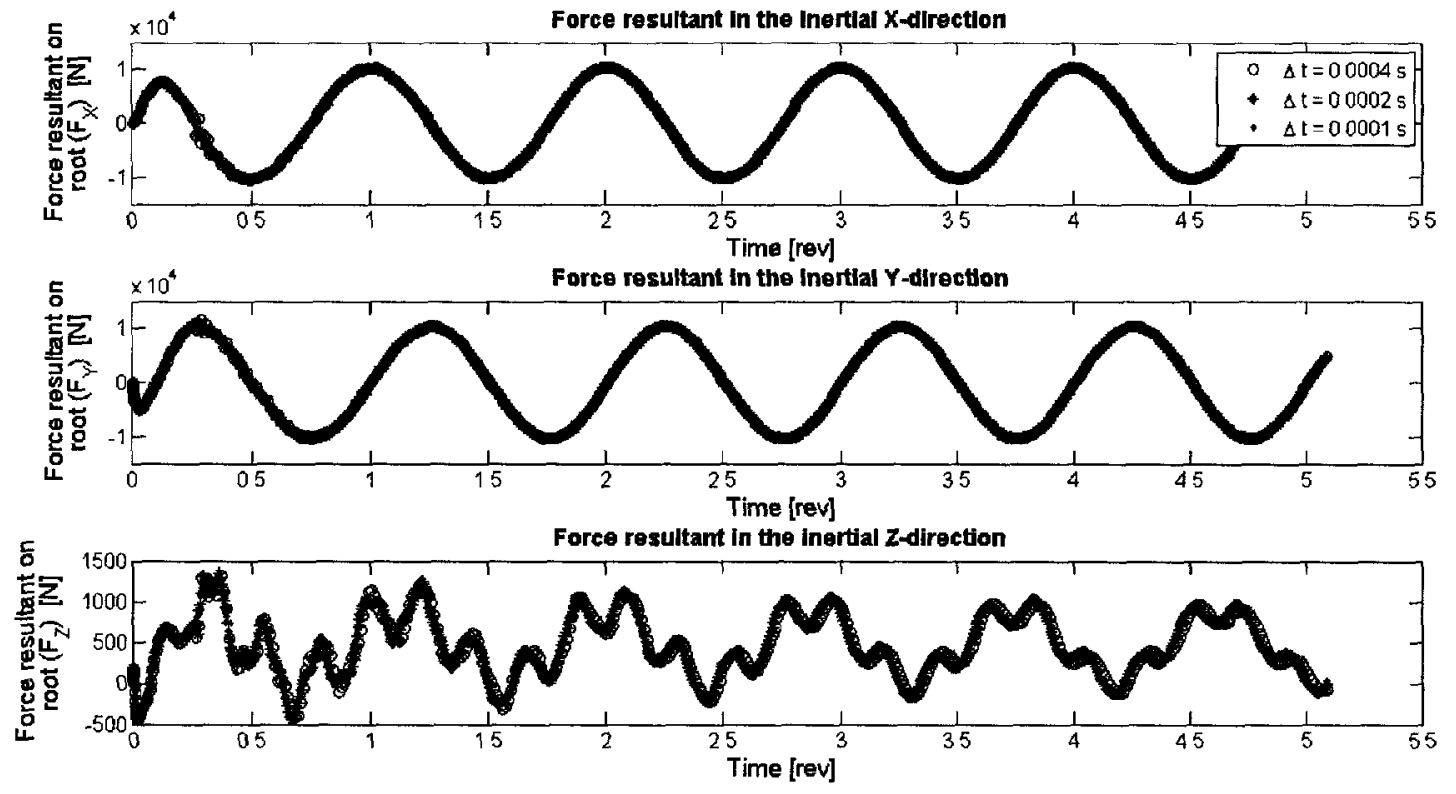

Figure 5.14: Resultant force vectors at the root of the helicopter blade.
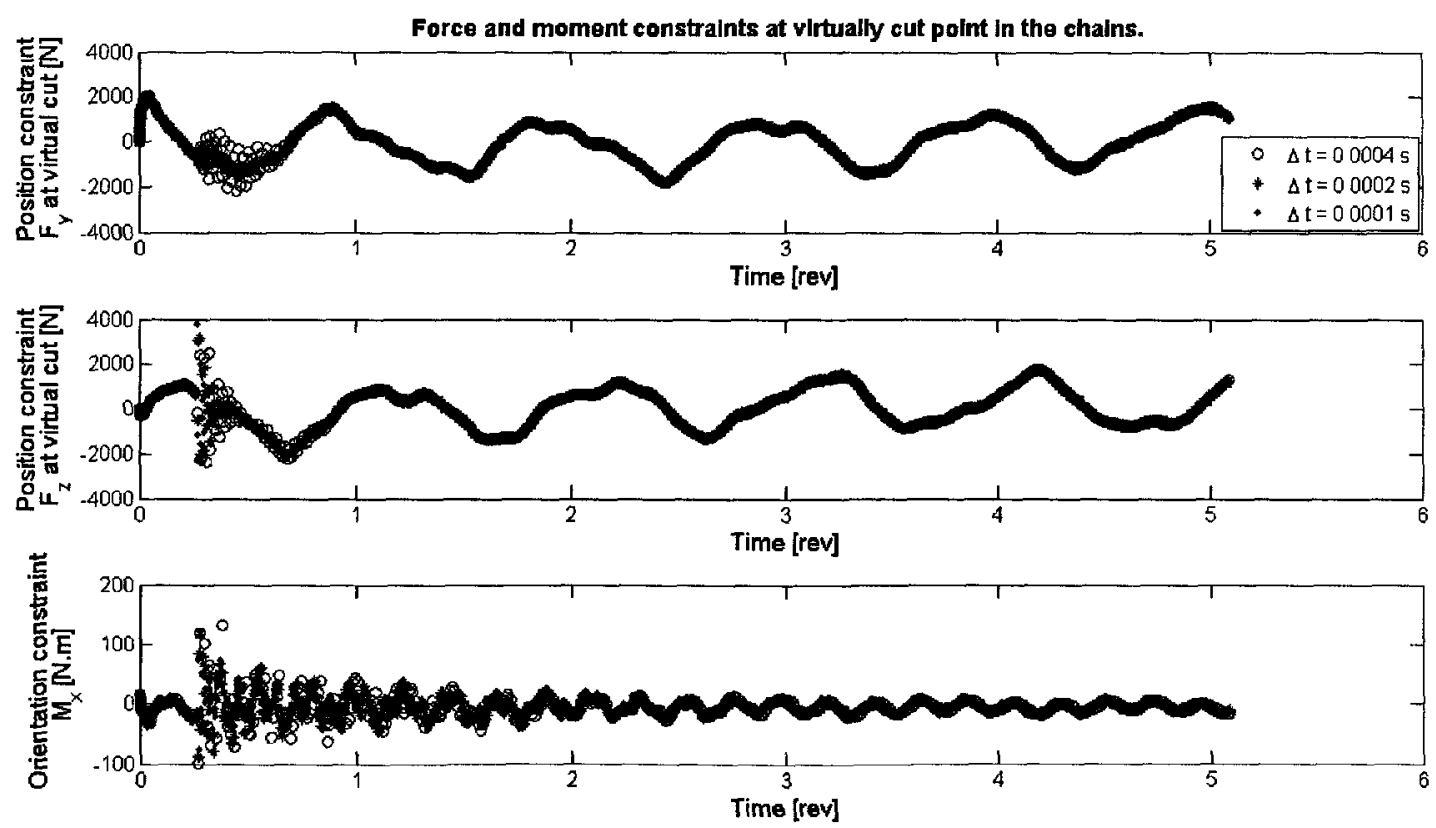

Figure 5.15: Resultant force vectors at the cut joint of the helicopter blade. 
used as a comparison of the accuracy of the simulation. A baseline test, with $\Delta t=$ $0.0001 \mathrm{~s}$ was used to compare the accuracy of the remaining timesteps. Timesteps of $\Delta t=0.001 \mathrm{~s}$, and $\Delta t=0.01 \mathrm{~s}$ were used to compare the simulation accuracy.

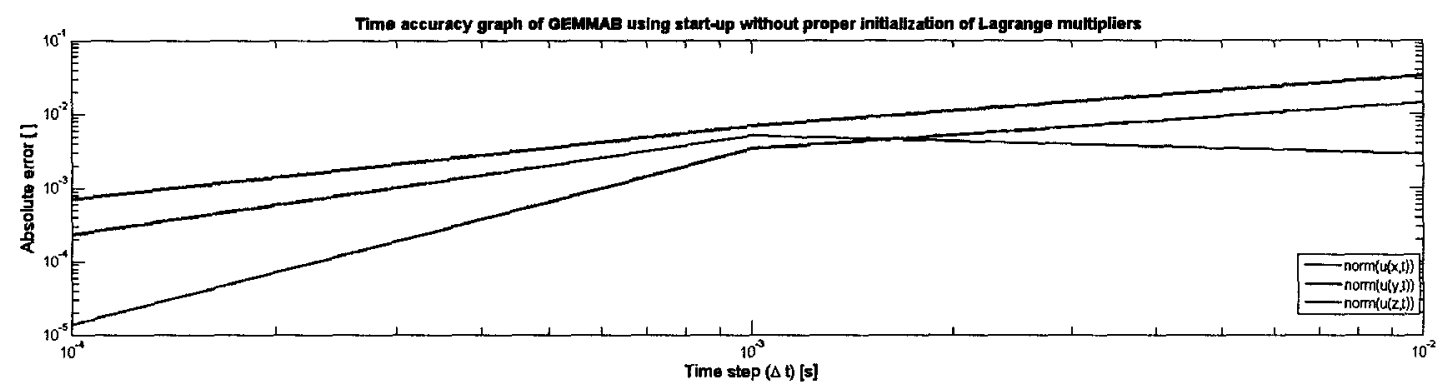

Figure 5.16: Convergence rates for orders of $\Delta t$ for the full articulated and elastic system.

The order of accuracy can be determined by finding the slope of these curves on the figure; for an order of magnitude change in the timestep, there should be two orders of magnitude in precision. The $x$-direction slope, for small time steps, seems to be closer to the second order convergence expected, but the other slopes are distinctly linear.

Unfortunately, Figure (5.16) does not show second order convergence as expected. It seems to show a linear convergence rate. This is probably caused by the incorrect initialization of the Lagrange multipliers in the computer code. For the second order convergence to be shown accurately, all variables must have appropriate initial conditions that make the system physically realistic. Potentially, the reason the $x$-direction norm looks to be closer to second order convergent is because there is less influence of the Lagrange multipliers at its initial position. 


\subsection{Validation of Complete Articulated Model}

The validation of the complete model is done with respect to the experimental results from testing the SHARCS blade at the NRC. The procedure is the same as in the verification, however, some of the hinge lengths have changed, and the initial positions of the hinges have also. Table 5.11 shows the changed link lengths, and Table 5.12 shows the new initial conditions. Note that for these tests, because the root pitch is set to zero, the lift force is small. The SHARCS blade was modified before this test, so it has the same twist distribution, but weighs less (approximately $380 \mathrm{~g}$ ) and testing was done at 1500 RPM, not 1555 RPM.

Table 5.11: Link properties for complete simulation verification.

\begin{tabular}{cccc}
\hline Link & $\mathrm{d}$ & $P_{L}$ & $P_{L_{2}}$ \\
\hline Length $[\mathrm{m}]$ & $1.766 \mathrm{E}-01$ & $1.766 \mathrm{E}-01$ & $1.766 \mathrm{E}-01$ \\
Radius $[\mathrm{m}]$ & $2.580 \mathrm{E}-02$ & $6.350 \mathrm{E}-03$ & $6.350 \mathrm{E}-03$ \\
Density $\left[\frac{\mathrm{kg}}{\mathrm{m}^{3}}\right]$ & $0.000 \mathrm{E}+00$ & $7.960 \mathrm{E}+03$ & $0.000 \mathrm{E}+00$ \\
\hline
\end{tabular}

Table 5.12: Hinge initial conditions, locked by user, based on trim hover conditions.

\begin{tabular}{cccccccc}
\hline Hinge & $\psi$ & $\beta$ & $\theta$ & $\theta_{1 s}$ & $\theta_{1 c}$ & $P_{L}$ & $P_{L_{2}}$ \\
\hline Angle [] & 0 & 0 & 0 & 0 & 0 & & \\
Length [m] & & & & & .1766 & .1766 \\
\hline${ }^{*}$ Calculated from theta_trim_hover.m & \\
\hline
\end{tabular}

Figure 5.17 shows the raw data from the experiment, and the discrete Fourier transform of the signal. Measurements were taken at $0.002 \mathrm{~s}$ intervals, which correspond to $18^{\circ}$ of azimuth rotation. As one can see, there are well defined peaks at integer multiples of the rotor speed. (A zoom on Figure 5.17 is shown in Figure 5.18 , to make the signal clearer.) This is unexpected, because the rigid rotor flapping 
blade is typically between the frequencies of $1.02 \Omega$ to $1.04 \Omega$ [6]. Figure 5.18 shows the simulation run from start-up for $0.5 \mathrm{~s}$ and then $1.5 \mathrm{~s}$ simulated time, along with the discrete Fourier transform of that signal. Similar to the above experimental results, the peaks are slightly less than the final rotor speed.

Table 5.13 shows the percent error of the simulation with respect to the first three recorded natural frequencies, using MATLAB to accurately pinpoint them, due to the large frequency sampling size. In general, the agreement is good between the experiment and the simulation: the first and second natural frequencies are within $4 \%$ of the rotor natural frequency and first harmonic. The resultant force, measured in the $z$-direction in the simulation, is on the same order of magnitude as the experimental results, even if the magnitude spectrum is not the same in both frequency plots. (This is due to the frequency sample rate, see below.) The parameterization of the model: friction damping, link lengths, masses, the blade mass and flexibility, etc; all have an effect on the forces.

Table 5.13: Link properties for complete simulation verification.

\begin{tabular}{lccc} 
& Experiment $\left[\frac{\mathrm{rad}}{\mathrm{s}}\right]$ & Simulation $\left[\frac{\mathrm{rad}}{s}\right]$ & Percent error [\%] \\
\hline$\omega_{n_{1}}$ & 157.1 & 163.4 & 3.91 \\
$\omega_{n_{2}}$ & 314.8 & 325.3 & 3.28 \\
$\omega_{n_{3}}$ & 472.5 & 540 & 13.3 \\
\hline
\end{tabular}

There is only a small error in the first two peaks, but the third is subject to a higher difference. This could be because of the inexact mass comparison. The mass is within $10 \%$ of the experimental mass from measurements, and will cause a shift of the natural frequencies. This explains why the power distribution is not congruent with the experimental results, as well: because the mass is shifted, so are the reasonant peaks and the magnitudes of the peaks will not match exactly. 

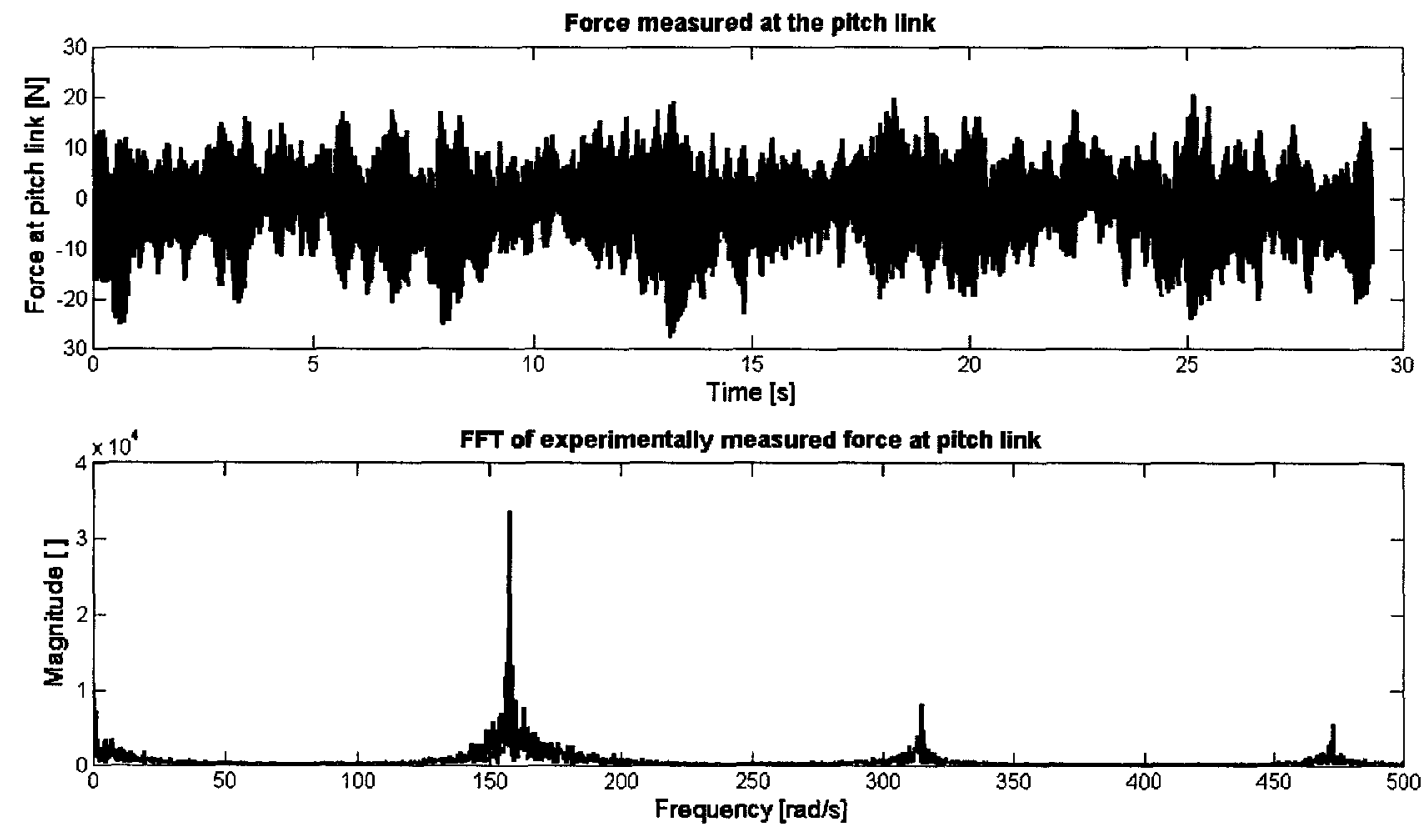

Figure 5.17: Experimentally measured pitch link forces and corresponding fast Fourier transform.

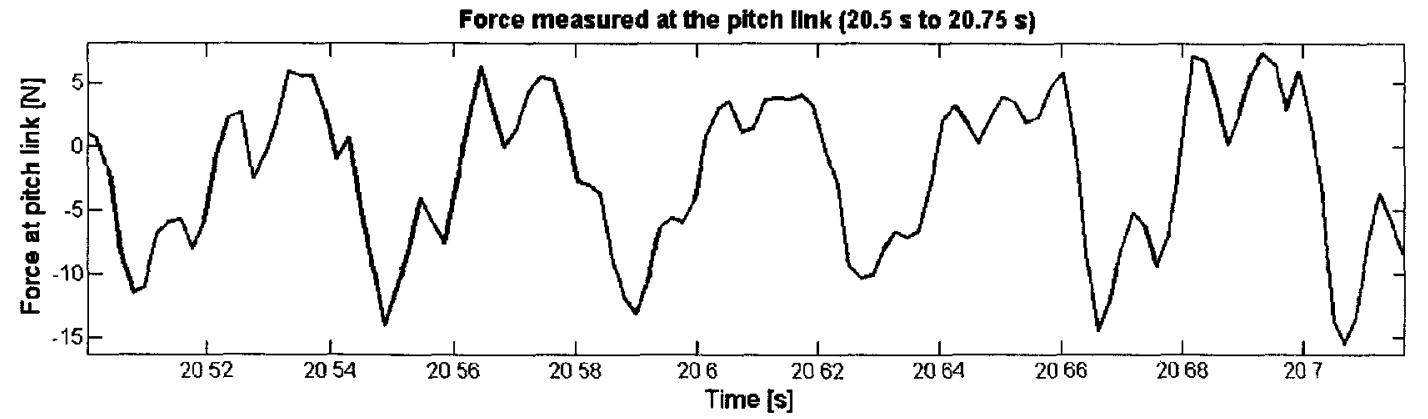

Figure 5.18: Experimentally measured pitch link forces, between $t=20.5 \mathrm{~s}$ and $t=20.75 \mathrm{~s}$. 

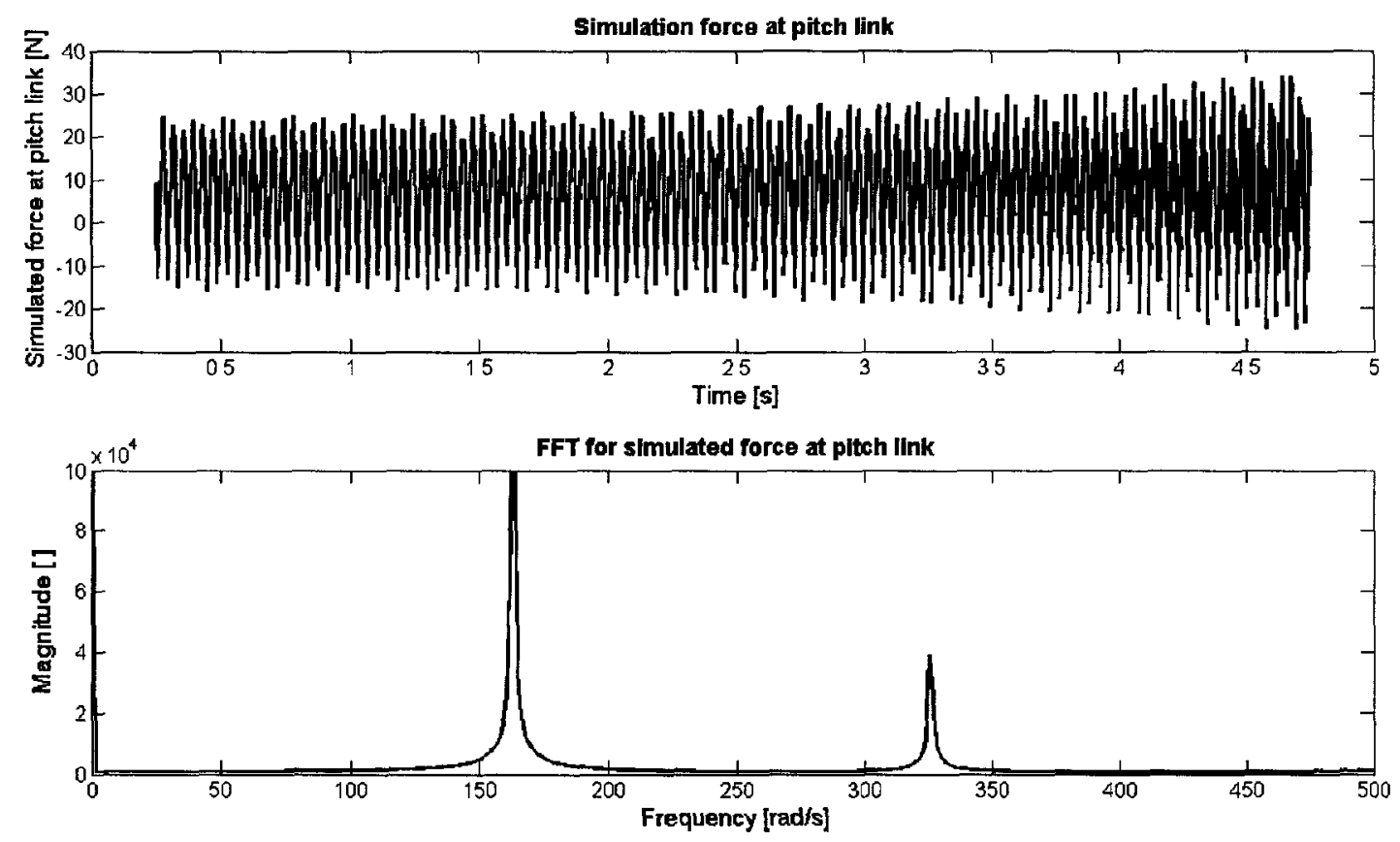

Figure 5.19: Simulated pitch link loads and corresponding Fourier transform.

Also, as can be seen in the simulation results, the higher harmonic vibrations dampen and the overall motion increases. This is due to the light numerical damping of the simulation, where $\rho_{\infty}=0.85$. The time step in the simulation is also quite high, $\Delta t=0.0008 s$, which reduced accuracy and power.

The simulation results are extremely dependent on the start up conditions. Changing the rotor start-up phase, the initial position of the flap hinge, the feathering hinge, and the swashplate tilt and displacement cause different results in terms of support forces generated, or displacements achieved. (This is somewhat expected, since it is a nonlinear model and it is path-dependent due to damping!) Some of the discrepancies between the experiment and the simulation can be attributed to the simulation parameters. In the physical experiment, the values for the damping within the rotor blade and on the hinges (friction) are not known, for the simulation, damping is not considered. Also, the experimental rotor blade is bolted at the blade root section, 
not considered in the elastic model, through two holes close to the quarter-chord. The blade root is contained within the clamp, which restricts the motion of the blade slightly. The lag degree of freedom is bolted to the rotor shaft, to prevent its motion; however, the effect of that bolt on the vibration characteristics is not studied in the simulation, nor is the friction or damping, from the screw tips securing the bolt, accounted in the simulation. Of course, the aerodynamic model will not match the real flow properly, since vortices are not considered, so the thrust will not be distributed properly over the blade.

\subsection{Control Efficacy}

This simulation uses the SHARCS blade in forward flight at $\mu=0.3 \mathrm{~s}$, so the swashplate tilt is the same as when testing the aerodynamics model with the experimental data. The articulation and blade arrangement is the same as before, in the test case to compare with the experimental results, except that all hinges are unlocked, save the swashplate degrees of freedom. The spring stiffnesses of the pitch link $\left(k_{P L}\right)$ and the actuator $\left(k_{c t l}\right)$ are based on values from initial research by the SHARCS group [22]. The spring stiffness values are $k_{P L}=175 \times 10^{3} \mathrm{Nm}$ and $k_{c t l}=50 \times 10^{3} \mathrm{Nm}$. However, the physical stiffness value of the APL system is always one of two values: $k_{P L}$ when the APL is not active, or $k_{P L}+k_{c t l}$ when it is.

Figure 5.20 shows the control forces applied to the pitch link, in the top plot. The bottom graph of the same Figure shows the pitch link stiffness throughout the duration of the simulation. The top graph in Figure 5.21 shows the difference in adaptive pitch link displacement with and without control, and the middle plot compares the feathering hinge oscillation. The change in motion at the pitch link and feathering hinge alters the motion of the rotor blade as well, and the bottom of Figure 5.21 

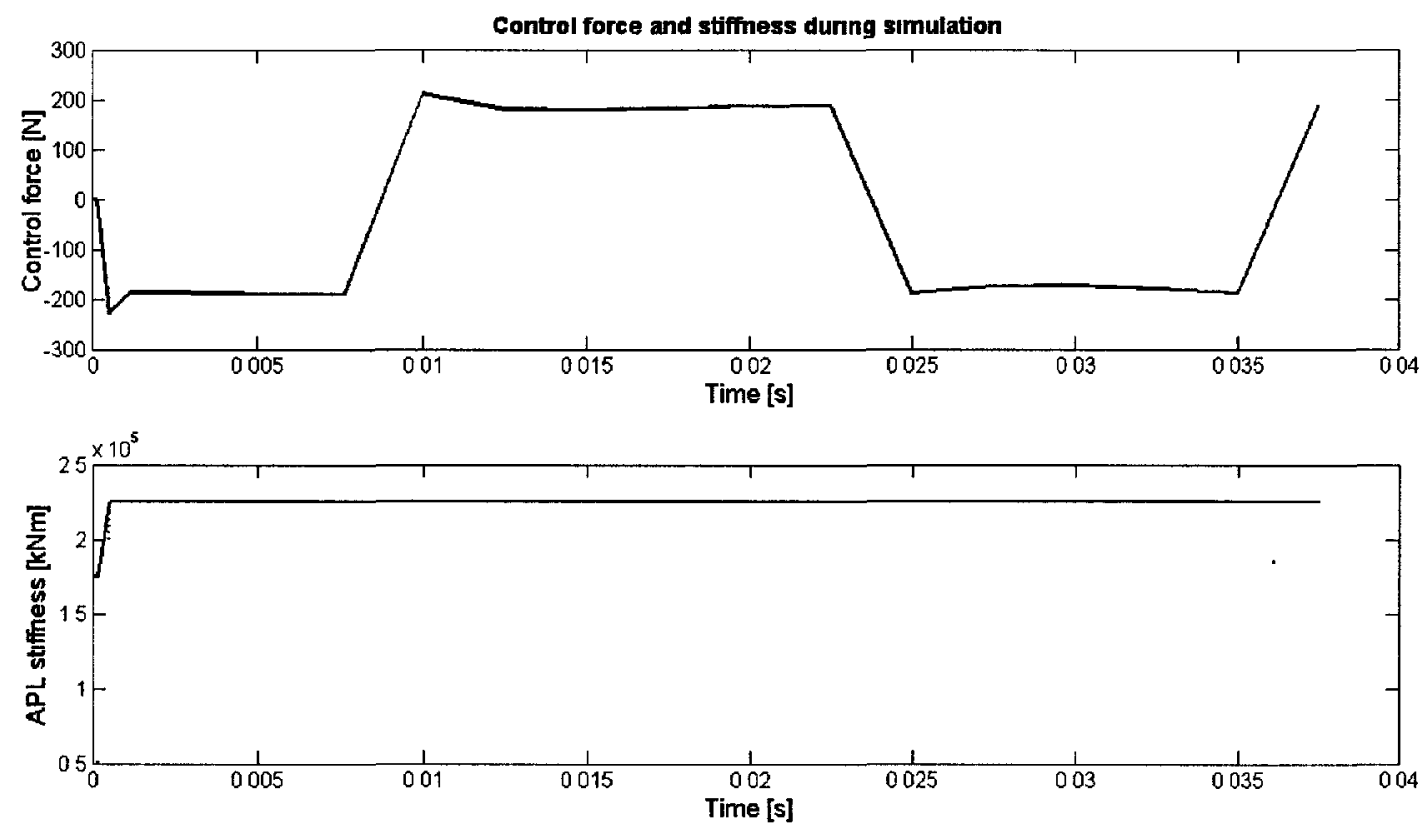

Figure 5.20: Control forces as a function of time and corresponding stiffness values at pitch link.

shows the neutral axis tip node trajectory in the flapping direction.

As these Figures show, the motion of the pitch link changes significantly with semiactive control. The motion can be damped (heat losses) also, which was not done for this specific simulation. However, based on these plots, vibration can be induced or reduced in the rotorcraft airframe, given the boundary conditions can stiffen or relax as decided by the control algorithm. The blade motion shows that there is a frequency shift in its response, which can also be useful to counter-act vibratory aerodynamic forces. Effectively, by changing the boundary conditions, the APL changes the energy distribution of the elastic body for which it attenuates vibration, and uses that energy to directly affect the excitation load. 

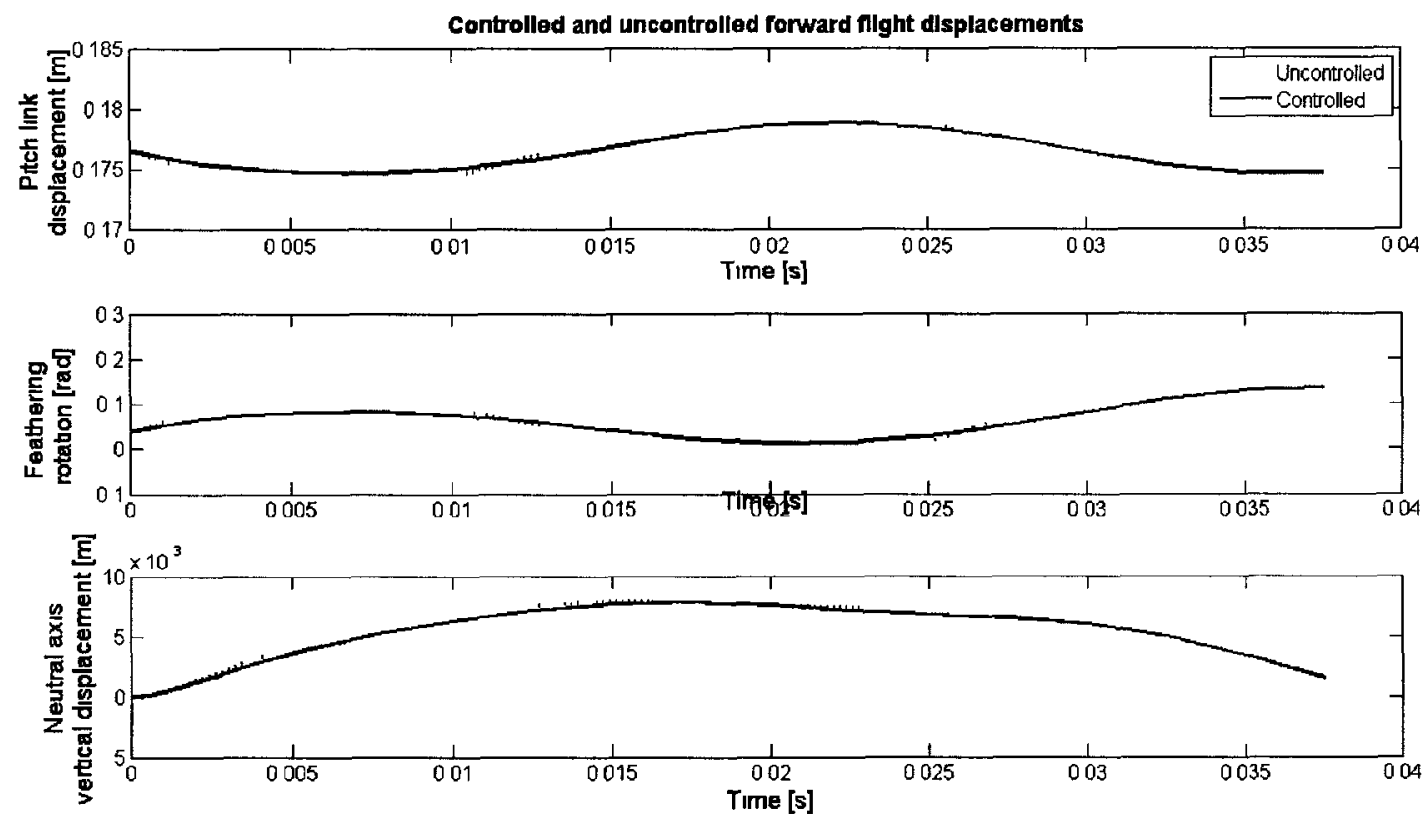

Figure 5.21: Comparing the displacement with and without control implemented 


\section{Chapter 6}

\section{Conclusions and Future Work}

A three-dimensional model using the generalized- $\alpha$ energy momentum method for nonlinear elastic material, with small strain deformations, is amalgamated with a non-weighted residual equation scheme for rigid body parallel kinematics. The combination of these models form the basis for a computer code capable of solving nonlinear, closed kinematic articulated helicopter hubs and elastic rotor blade dynamics. Added to the current program, the aerodynamics provide basic loading to emulate approximate hover and forward flight loads on the elastic rotor blade. The control algorithm allows a user to be able to implement control strategies by replacing the pitch link with an adaptive control device. The complete computer code exists as a tool for the SHARCS group to test new vibration control ideas and simulate results before experimentation.

Individual component functions have been independently verified and validated in the previous chapter. The three-dimensional model was compared to nonlinear beam models that have also been researched in the SHARCS group. The parallel kinematics have been examined by comparing it to a velocity stabilization method, which has

itself been validated using Runge-Kutta methods. Together, these two model components, the elastic and rigid body models, have been verified with simulations that 
show good convergence properties: in the displacement variables, elastic-rigid body coupling, and parallel chain constraint coupling. These numerical results were also compared to experimental measurements, and good agreement was obtained between the physical tests and the simulated model.

A rigid and elastic body coupled model was created with midpoint accelerations, allowing the GEMM time integration scheme and the Arnold and Brüls method to be combined. This was an important contribution to the overall analysis of combined rigd and elastic body systems, because it was a second order accurate method, with controllable dissipation, and allowed rigid motion of joints modelled with a quickly convergent two-step scheme. The figures showing the verification of the program show that the model is second order convergent in space, however, they seem to show that there is only linear convergence in time. This could be because the time steps were not small enough to show such behaviour properly.

Aerodynamics was included in the computer model, because it provided nonlinear external loads to give better agreement to previous experimental inflow data. Only hover loads were simulated for validation, as experimental data for forward flight with the SHARCS blade was unavailable. The control algorithm applied to forward flight simulation, which showed the ability of the control algorithm to alter the motion of the pitch link and blade. Both hover and forward flight models were included in the computer program; however, in the future, they should be replaced with the GENUVP model.

The control algorithm was instituted in the main program and it clearly showed the reduction of blade vibration. The control law diminished the motion of the blade, so for practical applications involving the reduction of vibration on the swashplate, it will need to be reversed.

To model the hub articulation properly, model drawings of the whirl-tower hub 
and blade attachment are necessary, which includes devices such as the lag bolt that restricted lag motion in the experiment. The appropriate values for dampeners and springs should also be measured or evaluated so that the structural model can be made more accurate. In particular, the motion dampers on the aerodynamic chain, especially lag, since it has little aerodynamic damping, should be measured and the values substituted into the code. The friction of the joints should be measured and a nonlinear friction law could easily be incorporated into the b3dsm.m code.

The swashplate hinge arrangement, in particular the spherical joint below the adaptive pitch link, should be modelled with the joint stops. In the physical system, the bearings of the joints connecting the swashplate and pitch link do not allow full revolutions, so that should be included in the structural model. Also, the play or backlash of the joints should be modelled, if possible.

The SHARCS blade model can be refined so that the simulated structural blade has the shape of the NACA airfoil. With this change, the proper mass distribution and elastic distribution could also be instituted. Because the current program is a three-dimensional model, one could create a shell model that would run faster than the full simulation presently does. A shell model would have the benefit of much faster analysis, and could be used to create a simulation that includes properties from composite laminae. Thus, a composite blade could be properly analysed with shell layers.

Also, longer simulations must be done to ensure that the system model is second order convergent in time. Currently, the models were tested with insufficiently small time steps, so the convergence rate is not well shown. Because one of the significant contributions of the thesis is the coupling of the rigid and elastic body models, it must be checked to guarantee the preservation of second order convergence.

The next step for this particular model is its inclusion in SMARTROTOR, or at 
least its coupling with the GENUVP aerodynamics model. GENUVP currently does not allow structural rigid body motion, so coupling the current structural model with the aerodynamic model from GENUVP will create a powerful analysis tool, even if the control algorithm is ignored.

Improvements to the control algorithm may be necessary, if there is a desire to control specific modes of vibration, those that do not cancel with symmetric blades, for instance, especially if the pilot control inputs are time-dependent. In such a case, the control law would need to be changed to ensure tha the pilot commands are not cancelled as undesired harmonic vibration. The viscous friction damping caused by heat loss was not modelled, so that will undoubtedly attenuate vibration transmission through the APL, if instituted. Also, the control algorithm, as implemented in the main code, controls the vibration of the rotor blade, not the fuselage. In fact, this increases the vibration of the cabin, so it must be modified to attenuate vibration transmission.

Finally, the control of the vibratory forces while implementing the other SHARCS concepts on the rotor blade can soon be simulated. Truly testing the entire SHARCS concept will come when all three systems can be implemented physically, but simulating the systems in concert is important to fine-tune the vibration and noise suppression. 


\section{List of References}

[1] B. G. van der Wall, "2nd HHC aeroacoustic rotor test (HART II) - part 1: Test documentation," Tech. Rep. IB-111-2003/31, German Aerospace Centre (DLR), Braunschweig, Germany, 2003.

[2] E. J. Lovesey, "The helicopter - some ergonomic factors," Journal of Applied Ergonomics, vol. 6(3), pp. 139-146, 1975.

[3] J. G. Leishman, Principles of Helicopter Aerodynamics. Cambridge, UK: Cambridge Aerospace Press, 2000.

[4] I. Papavassiliou et al, "Coupled rotor-fuselage vibration reduction using openloop blade pitch control," Mathematical Computational Modelling, vol. 18(3/4), pp. 131-156, 1993.

[5] A. Rosen and R. Ben-Ari, "Mathematical modelling of a helicopter rotor track and balance: Theory," Journal of Sound and Vibration, vol. 200(5), pp. 589603, 1997.

[6] A. R. S. Bramwell, Helicopter Dynamics. London, UK: Edward Arnold, 1976.

[7] F. Straub et al, "Development of a piezoelectric actuator for trailing edge flap control of full scale rotor blades," Journal of Smart Materials and Structures, vol. 10(1), pp. 25-34, 2001.

[8] T. Harold, "Performance characterization of the smart spring concept for indirect-active vibration suppression," Master's thesis, Carleton University, Ottawa, Canada, 2004.

[9] Y. H. Yu et al, "Reduction of helicopter blade-vortex interaction noise by active rotor control technology," Progress in Aerospace Sciences, vol. 33, pp. 647-687, 1997. 
[10] N. D. Ham, "Helicopter individual-blade-control reserach at MIT 1977-1985," Vertica, vol. 11(1/2), pp. 109-122, 1987.

[11] S. Jacklin, et al, "Reduction of helicopter bvi noise, vibration and power consumption through individual blade control," Proceedings of the $50^{\text {th }}$ Annual Forum of the American Helicopter Society, pp. 579-596, 1994.

[12] S. Jacklin, et al, "Reduction of helicopter bvi noise, vibration and power consumption through individual blade control," Proceedings of the $51^{\text {st }}$ Annual Forum of the American Helicopter Society, pp. 662-680, 1995.

[13] R. Kube and B. G. van der Wall, "Effects on bvi noise and vibrations - a combined numerical and experimental investigation," Proceedings of the $55^{\text {th }}$ International Annual Forum of the American Helicopter Society, pp. 2282-2291, 1999.

[14] F. Nitzsche and E. Breitbach, "Using adaptive structures to attenuate rotary wing aeroelastic response," Journal of Aircraft, vol. 31(5), pp. 1178-1188, 1994.

[15] M. Garfinkle, "Smarter rotor-blade composite 'smart' spar," Materials and Design, vol. 15(1), pp. 27-31, 1994.

[16] A. Büter and E. Breitbach, "Adaptive blade twist - calculations and experimental results," Aerospace Science and Technology, vol. 4, pp. 309-319, 2000.

[17] C. E. S. Cesnik, et al, "Dynamic response of active twist rotor blades," Journal of Smart Materials and Structures, vol. 10, pp. 62-76, 2001.

[18] F. Afagh, F. Nitzsche, and N. Morozova, "Dynamic modelling and stability of hingeless helicopter blades with a smart spring," The Aeronautical Journal of the Royal Aeronautical Society, pp. 369-377, 2004.

[19] R. Beaubien, "Rotorcraft research group: Carleton university." Online: http://rotorcraft.mae.carleton.ca/, 2005. Homepage of the Carleton University rotorcraft research group, led by Professors Nitzsche and Feszty.

[20] K. Khomutov, "Design, analysis and instrumentation of the SHARCS rotor blade with three actively controlled systems," Master's thesis, Carleton University, Ottawa, ON, Canada, 2009.

[21] M. Cha, "Feasibility study and design of an actively controlled tip for helicopter rotor blades," Master's thesis, Carleton University, Ottawa, ON, Canada, 2007. 
[22] M. Ghorashi et al, "Prelminary design of a scaled rotor blade with vibration and noise control devices," 32 $2^{\text {nd }}$ European Rotorcraft Forum, vol. AR-08, 2006.

[23] D. Ülker et al, "SHARCS: Hybrid control concept of vibration reduction of helicopters," 34 $4^{\text {th }}$ European Rotorcraft Forum, vol. 9A1, 2008.

[24] D. Ülker, A New Framework For Helicopter Vibration Suppression: TimePeriodic Identification and Controller Design. PhD thesis, Carleton University, Ottawa, ON, Canada, 2011.

[25] D. Gransden et al, "Development of nonlinear elastic bending and torsion of articulated rotor blades with an impedance control device replacing the common pitch link," 31 $3{ }^{\text {st }}$ European Rotorcraft Forum, 2005.

[26] F. Nitzsche and G. Oxley, "Smart spring control of vibration and noise in helicopter blades," 46 ${ }^{\text {th }}$ AIAA/ASCE/AHS/ASC Structures, Structural Dynamics and Materials Conference, pp. 2005-2027, 2005.

[27] J. C. Houbolt and G. W. Brooks, "Differential equations of motion for combined flapwise bending, chorwise bending and torsion of twisted nonuniform rotor blades," Tech. Rep. 1346, NASA, Washington DC., 1958.

[28] D. H. Hodges and E. H. Dowell, "Nonlinear equations of motion for the elastic bending and torsion of twisted nonuniform rotor blades," Tech. Rep. D-7818, NASA, Washington DC., 1974.

[29] W. Johnson, Helicopter Theory. Princeton, New Jersey, USA: Princeton University Press, 1980.

[30] T. Yokoyama, "Free vibration characteristics of rotating Timoshenko beams," International Journal of Mechanical Sciences, vol. 30(10), pp. 743-755, 1988.

[31] M. Borri, "Helicopter rotor dynamics by finite element time approximation," Computers and Mathematics with Applications, vol. 12(1.1), pp. 149-160, 1986.

[32] B. O. Al-Bedoor and Y. A. Khulief, "Vibrational motion of an elastic beam with prismatic and revolute joints," Journal of Sound and Vibration, vol. 190(2), pp. 195-206, 1996.

[33] S. R. Turnour and R. Celi, "Modeling of flexible rotor blades for helicopter flight dynamics," Journal of the American Helicopter Society, vol. 41(1), pp. 52-61, 1996. 
[34] B. O. Al-Bedoor, "Dynamics model of coupled shaft torsional and blade bending deformations in rotors," Computer Methods in Applied Mechanics and Engineering, vol. 169, pp. 177-190, 1999.

[35] H. H. Yoo and S. H. Shin, "Vibration analysis of rotating cantilever beams," Journal of Sound and Vibration, vol. 212(5), pp. 807-828, 1998.

[36] J. Chung and H. H. Yoo, "Dynamics analysis of a rotating cantilever beam by using the finite element method," Journal of Sound and Vibration, vol. 249(1), pp. $147-164,2002$.

[37] Z. C. Zheng, G. Ren, and Y. M. Cheng, "Aeroelastic responce of a couple rotor/fuselage system in hovering and forward flight," Archive of Applied Mechanics, vol. 69, pp. 68-82, 1999.

[38] D. H. Hodges and R. A. Ormiston, "Stability of elastic bending and torsion of uniform cantilever rotor blades in hover with variable structural coupling," Tech. Rep. D-8192, NASA, Washington DC., 1976.

[39] K. V. Truong, "Dynamics studies of the ERATO blade, based on finite element analysis," 31 $1^{\text {st }}$ European Rotorcraft Forum, pp. 94.1-94.8, 2005.

[40] M. Das, E. Madenci, and F. K. Straub, "Aeroelastic analysis of rotor blades using three dimensional flexible multibody dynamic analysis," AIAA/ASME/ASCE/AHS Structures, Structural Mechanics and Materials Conference, pp. 7206-7215, 2007.

[41] J. Bonet and R. Wood, Nonlinear continuum mechanics for finite element analysis. Cambridge, UK: Cambridge University Press, 1997.

[42] Y. Haddad, Mechanical Behaviour of Engineering Materials, vol. 1. Dorchester, NL: Kluwer Academic Publishers, 2000.

[43] T. Mura and T. Koya, Variational Methods in Mechanics. New York: Oxford University Press, 1992.

[44] M. Crisfield, Non-linear Finite Element Analysis of Solids and Structures, Vol. 1: Essentials. Chichester, UK: John Wiley and Sons, 1991.

[45] M. Crisfield, Non-linear Finite Element Analysis of Solids and Structures, Vol. 2: Advanced Topics. Chichester, UK: John Wiley and Sons, 1997. 
[46] B. Bornemann and W. A. Wall, Finite Element Method. Munich, Germany: Lehrstuhl für Numerische Mechanik, 2006.

[47] B. Bornemann and W. A. Wall, Nichtlineare Finite-Element-Methoden. Munich, Germany: Lehrstuhl für Numerische Mechanik, 2007.

[48] D. Kuhl and M. A. Crisfield, "Energy-conserving and decaying algorithms in non-linear structural dynamics," International Journal for Numerical Methods in Engineering, vol. 45, pp. 569-599, 1999.

[49] S. Niku, Introduction to Robotics: Analysis, Systems, Applications. San Luis Obispo, California: Prentice Hall, 2001.

[50] L. Sciavicco and B. Siciliano, Modelling and Control of Robot Manipulators. London: Springer-Verlag, 3rd ed., 2002.

[51] G. Strang, Linear Algebra and its Applications. New York: Academic Press, 1976.

[52] G. Strang, Introduction to Applied Mathematics. Wellesley, Massachusetts, USA: Wellesley-Cambridge Press, 1986.

[53] K.-J. Bathe, Finite Element Procedues. Englewood Cliffs, New Jersey, USA: Prentice Hall, 1996.

[54] A. A. Shabana, Computational Dynamics. New York: John Wiley and Sons, Inc., 2nd ed., 2001.

[55] J. N. Reddy and M. L. Rasmussen, Advanced Engineering Analysis. New York: John Wiley and Sons, 1982.

[56] O. Agrawal, "General approach to dynamic analysis of rotorcraft," Journal of Aerospace Engineering, vol. 4(1), pp. 91-107, 1991.

[57] W. Johnson, CAMRAD II: Comprehensive Analytical Model of Rotorcraft Aerodynamics and Dynamics. Palo Alto, CA, USA: Johnson Aeronautics, 2000.

[58] O. A. Bauchau, DYMORE User's Manual. Georgia Institute of Technology, Atlanta, 2007.

[59] A. Rosen, R. G. Loewy, and R. B. Mathew, "Unified treatment for dealing with auxiliary conditions in blade dynamics," AIAA Journal, vol. 29(6), pp. 968976, 1990. 
[60] R. G. Loewy et al, "Dynamic analysis of rotor blades with root retention design variations," AIAA Journal of Aircraft, vol. 29(5), pp. 782-789, 1992.

[61] O. Bauchau and N. K. Kang, "A multibody formulation for helicopter structural dynamic analysis," American Helicopter Society, vol. 38(22), pp. 3-14, 1993.

[62] O. Bauchau, "Multi-body formulation for rotorcraft dynamic analysis," American Helicopter Society 51 ${ }^{\text {st }}$ Annual Forum, vol. 2, pp. 1788 - 1798, 1995.

[63] A. Laulusa and O. Bauchau, "Review of classical approaches for constraint enforcement in multibody systems," Journal of Computation and Nonlinear Dynamics, vol. 3(1), pp. 11004.1-11004.8, 2008.

[64] O. Bauchau, C. Bottasso, and Y. Nikishkov, "Modeling rotorcraft dynamics with finite element multibody procedures," Mathematical and Computer Modeling, vol. 33, pp. 1113-1137, 2001.

[65] J. Y. S. Luh and Y.-F. Zheng, "Computation of input generalized forces for robots with closed kinematic chain mechanisms," IEEE Journal of Robotics and Automation, vol. 1(2), pp. 95-103, 1985.

[66] Y.-F. Zheng and J. Y. S. Luh, "Joint torques for control of two coordinated moving robots," tech. rep., IEEE International Conference on Robotics and Automation, San Francisco, CA, 1986.

[67] C. W. Gear, B. Leimkuhler, and G. K. Gupta, "Automatic integration of EulerLagrange equations with constraints," Journal of Computational and Applied Mathematics, vol. 12/13, pp. 77-90, 1985.

[68] J. J. Murray and G. H. Lovell, "Dynamic modelling of closed chain robotic manipulators and implications for trajectory control," IEEE TRANSACTIONS ON ROBOTICS AND AUTOMATION, vol. 5(4), pp. 522-528, 1989.

[69] S.-K. Lin, "Dynamics of the manipulator with closed chains," IEEE Transactions on Robotics and Automation, vol. 6(4), pp. 496-501, 1990.

[70] I. Bindzi, et al., "Dynamic analysis of robotic manipulators with closed kinematic chains," IEEE Region 10 Conference, vol. Tencon 92, pp. 639-643, 1992.

[71] W. Blajer, "State and energy stabilization in multibody systems," Mechanics Research Communications, vol. 26(3), pp. 261-268, 1999. 
[72] W. Blajer, "Elimination of constraint violation and accuracy aspects in numerical simulation of multibody systems," Multibody System Dynamics, vol. 7, pp. 265-284, 2002.

[73] J. Kövecses, J.-C. Piedboeuf, and C. Lange, "Dynamics modeling and simulation of constrained robotic systems," IEEE/ASME Transactions on Mechatronics, vol. 8(2), pp. 165-177, 2003.

[74] A. Śmiarowski and J. N. Anderson, "On Jacobians for robots containing closed kinematic chains," Proceedings from the $20^{\text {th }}$ Southeastern Symposium on System Theory, pp. 464-467, 1988.

[75] D. Negrut, et al, "Method in the context of index 3 differential algebraic equations of multibody dynamics," Internation Journal for Numerical Methods in Engineering, vol. 00:1-6, pp. 1-25, 2000.

[76] C. Lunk and B. Simeon, "Solving constrained mechanical systems by the family of Newmark and $\alpha$-methods," Journal of Applied Mathematics and Mechanics, vol. 86(10), pp. 772-784, 2006.

[77] N. Khude, et al, "A discussion of low order numerical integration formulas for rigid and flexible multibody dynamics," Proceedings of the $6^{\text {th }}$ ASME International Conference on Multibody Systems, Nonlinear Dynamics and Control, vol. IEDTC07, pp. 1-12, 2007.

[78] M. Arnold and O. Brüls, "Convergence of the generalized- $\alpha$ scheme for constrained mechanical systems," Multibody System Dynamics, vol. 18, pp. 185$202,2007$.

[79] D. Opoku, "Aeroelastic and aeroacoustic modelling of rotorcraft," Master's thesis, Carleton University, Ottawa, Canada, 2002.

[80] J. Beale and A. Majda, "High order accurate vortex methods with explicit velociy kernels," Journal of Computational Physics, vol. 58, pp. 188-208, 1985.

[81] S. G. Voutsinas, "A generalized unsteady vortex particle method for solving the unsteady flow around multi-component configurations," tech. rep., NTUA, Athens, Greece, 1990.

[82] S. G. Voutsinas et al, "Investigation of yawed operation of wind turbines by means of a vortex particle method," AGARD-CP-552 FDP Symposium on Aerodynamics and Aeroacoustics of Rotorcraft, no. 11, 1995. 
[83] S. G. Voutsinas and D. G. Triatos, "High-resolution aerodynamic analysis of full helicopter configurations," Twenty-fifth European Rotorcraft Forum, no. C11, pp. 188-208, 1999.

[84] D. G. Triantos, Aerodynamic and Aeroacoustic Analysis of Helicopter Rotors. $\mathrm{PhD}$ thesis, National Technical University of Athens, Athens, Greece, 2002.

[85] K. W. Mangler and H. B. Squire, "The induced velocity field of a rotor," Tech. Rep. No. 2642, A. R. C. Technical Report, Aeronautical research council reports and memoranda, London, UK, 1953.

[86] J. W. Elliott and S. L. Althoff, "Inflow measurement made with a laser velocimeter on a helicopter model in forward flight: Volume i rectangular planform blades at an advance ratio of 0.15," Tech. Rep. 100541, NASA, Langley Research Center, VA, USA, 1988.

[87] J. W. Elliott and S. L. Althoff, "Inflow measurement made with a laser velocimeter on a helicopter model in forward flight: Volume i rectangular planform blades at an advance ratio of 0.23," Tech. Rep. 100542, NASA, Langley Research Center, VA, USA, 1988.

[88] J. W. Elliott and S. L. Althoff, "Inflow measurement made with a laser velocimeter on a helicopter model in forward flight: Volume i rectangular planform blades at an advance ratio of 0.30," Tech. Rep. 100543, NASA, Langley Research Center, VA, USA, 1988.

[89] D. Hodges, "Vibration and response of non-uniform rotating beams with discontinuities," Journal of the American Helicopter Society, vol. 25(5), pp. 43-50, 1979 .

[90] D. Hodges, "An approximate formula for the fundamental frequency of a uniform rotating beam clamped off the axisof rotation," Journal of Sound and Vibration, vol. 77(1), pp. 11-18, 1981.

[91] S. K. Biswas and F. D. Klafter, "Dynamic modeling and optimal control of flexible robotic manipulators," International Conference on Robotics and Automation, vol. 1, pp. 15-20, 1988.

[92] H. Diken, "Vibration control of a rotating Euler-Bernoulli beam," Journal of Sound and Vibration, vol. 232(3), pp. 541-551, 2000. 
[93] M. Dadfarnia et al, "An observer-based piezoelectic control of flexible cartesian robot arms: theory and experiment," Control Engineering Practice, vol. 12, pp. 1041-1053, 2004.

[94] S.-B. Choi and M. Han, "Vibration control of a rotating cantilevered beam using piezoactuators: Experimental work," Journal of Sound and Vibration, vol. 277, pp. 436-442, 2004.

[95] S. Na, L. Librescu, and J. K. Shim, "Modeling and bending vibration control of nonuniform thin-walled rotating beams incorporating adaptive capabilities," International Journal of Mechanical Sciences, vol. 45, pp. 1347-1367, 2003.

[96] F. Nitzsche and E. Breitbach, "The smart structures technology in the vibration control of helicopter blades in forward flight," First European Conference on Smart Structures and Materials, pp. 312-324, 1992.

[97] F. Nitzsche and E. Breitbach, "Individual blade control of hinged blades using smart structures," Journal of Smart Materials and Structures, vol. 3(2), pp. 171-180, 1994.

[98] F. Nitzsche, "Tailoring the dynamic response of helicopter rotors using smart materials," Proceedings of the AIAA/ASME Adaptive Structures Forum, vol. AIAA 95-1083, 1995.

[99] F. Nitzsche, "A new individual blade control approach to attenuate rotor vibration," Sixth International Conference on Adaptive Structures, pp. 230-240, 1996.

[100] F. Nitzsche, "Aeroelastic analysis of a helicopter rotor blade with active impedance control at the root," Canadian Aeronautics and Space Journal, vol. $47(1)$, p. $7,2001$.

[101] L. Lehman, Hybrid state vector methods for structural dynamic and aeroelastic boundary value problems. $\mathrm{PhD}$ thesis, Stanford University, California, USA, 1982.

[102] P. Anusonti-Inthra and F. Gandhi, "Helicopter vibration reduction through cyclic variations in rotor blade root stiffness," Journal of Intelligent Material Systems and Structures, vol. 11, pp. 153-166, 2000. 
[103] P. Anusonti-Inthra and F. Gandhi, "Optimal control of helicopter vibration through cyclic variations in blade root stiffness," Journal of Smart Materials and Structures, vol. 10, pp. 86-95, 2001.

[104] F. Gandhi and P. Anusonti-Inthra, "Helicopter vibration reduction using discrete controllable-stiffness devices at the rotor hub," AIAA Journal of Aircraft, vol. 39(4), pp. 668-677, 2002.

[105] P. Anusonti-Inthra and F. Gandhi, "Adaptive control of semiactive variable stiffness devices for narrow-band disturbance rejection," Journal of Intelligent Material Systems and Structures, vol. 14, pp. 191-201, 2003.

[106] P. Anusonti-Inthra, Semi-Active Control of Helicopter Vibration Using Controllable Stiffness and Damping Devices. PhD thesis, Pennsylvania State University, Pennsylvania, USA, 2002.

[107] J. B. Yang, L. J. Jiang, and D. C. Chen, "Dynamic modelling and control of a rotating Euler-Bernoulli beam," Journal of Sound and Vibration, vol. 274, pp. 863-875, 2004.

[108] K. A. Cunefare, "State-switched absorber for vibration control of point-excited beams," Journal of Intelligent Material Systems and Structures, vol. 13(2), pp. $97-105,2002$.

[109] Y. Liu, T. P. Waters, and M. J. Brennan, "A comparison of semi-active damping control strategies for vibration isolation of harmonic disturbances," Journal of Sound and Vibration, vol. 280, pp. 21-39, 2005.

[110] L. für Numerische Mechanik, "Technical report on baci," tech. rep., Technical University of Munich, Munich, Germany, 2008.

[111] T. J. R. Hughes, The Finite Element Method. San Luis Obispo, California: Prentice Hall, 1987.

[112] J. C. Simo and N. Tarnow, "The discrete energy-momentum method: conserving algorithms for nonlinear elastodynamics," Journal of Applied Mathematics and Physics, vol. 43, pp. 757-792, 1992.

[113] J. C. Simo and N. Tarnow, "A new energy and momentum conserving algorithm for the nonlinear dynamics of shells," International Journal for Numerical Methods in Engineering, vol. 37(15), pp. 2527-2549, 1994. 
[114] J. C. Simo, N. Tarnow, and M. Doblaré, "Non-linear dynamics of threedimensional rods: exact energy and momentum conserving algorithms," International Journal for Numerical Methods in Engineering, vol. 38, pp. 1431-1473, 1995.

[115] J. Denavit and R. Hartenberg, "A kinematic notation for lower-pair mechanisms based on matrices," ASME Journal of Applied Mechanics, vol. 23, pp. 215-221, 1955.

[116] A. Eugene and T. Baumeister, Marks' Standard Handbook for Mechanical Engineers. New York: McGraw-Hill, 1996.

[117] B. Simeon, "On lagrange multipliers in flexible multibody dynamics," Computer Methods in Applied Mechanics and Engineering, vol. 195, pp. 6993-7005, 2006.

[118] C. Bottasso, D. Dopico, and L. Trainelli, "On the optimal scaling of index three differential algebraic equations in multibody dynamics," Multibody System Dynamics, vol. 19, pp. 3-20, 2008.

[119] M. Ghorashi, Dynamics of Elastic Nonlinear Rotating Composite Beams with Embedded Actuators. PhD thesis, Carleton University, Ottawa, ON, Canada, 2009. 